Florida International University FIU Digital Commons

\title{
A Methodology to Estimate Time Varying User Responses to Travel Time and Travel Time Reliability in a Road Pricing Environment
}

Patricio A. Alvarez

Florida International University, palva002@fiu.edu

DOI: $10.25148 /$ etd.FI12050237

Follow this and additional works at: https://digitalcommons.fiu.edu/etd

\section{Recommended Citation}

Alvarez, Patricio A., "A Methodology to Estimate Time Varying User Responses to Travel Time and Travel Time Reliability in a Road Pricing Environment" (2012). FIU Electronic Theses and Dissertations. 631.

https://digitalcommons.fiu.edu/etd/631 


\section{FLORIDA INTERNATIONAL UNIVERSITY}

Miami, Florida

A METHODOLOGY TO ESTIMATE TIME VARYING USER RESPONSES TO

TRAVEL TIME AND TRAVEL TIME RELIABILITY IN A ROAD PRICING ENVIRONMENT

A dissertation submitted in partial fulfillment of the

requirements for the degree of

DOCTOR OF PHILOSOPHY

in

CIVIL ENGINEERING

by

Patricio Alvarez 


\section{To: Dean Amir Mirmiran}

College of Engineering and Computing

This dissertation, written by Patricio Alvarez, and titled A Methodology to Estimate Time Varying User Responses to Travel Time and Travel Time Reliability in a Road Pricing Environment, having been approved in respect to style and intellectual content, is referred to you for judgment.

We have read this dissertation and recommend that it be approved.

$\begin{array}{r}\hline \text { Albert Gan } \\ \hline \text { L. David Shen } \\ \hline \text { Zhenmin Chen } \\ \hline \text { Mohammed Hadi, Major Professor }\end{array}$

Date of Defense: March 29, 2012

The dissertation of Patricio Alvarez is approved.

Dean Amir Mirmiran
College of Engineering and Computing

Florida International University, 2012 


\section{DEDICATION}

I dedicate this thesis to Carolina, Bastian, Diego and Francisca; they came to my life to make it a whole. Without their patience, understanding, support; and most of all love, the completion of this work would not have been possible. 


\section{ACKNOWLEDGMENTS}

This dissertation would not have been possible without the guidance of my committee members, help from friends and several individuals who in one way or another contributed and extended their valuable assistance in the preparation and completion of this study.

First and foremost, I would like to express my deepest gratitude to my advisor, Dr. Mohammed Hadi for his excellent guidance, caring, patience correcting my writing, financially supporting it and providing me with an excellent atmosphere for doing research.

Also, I would like to thank Dr. David Shen, Dr. Albert Gan and Dr. Zhenmin Chen who helped me developing a background in Transportation Engineering and kindly provided comments about my research

Finally, I would also like to thank Dr. Yan Xiao and Dr. Halit Ozen who besides providing thoughtful comments, gave me the privilege to share their friendship. 


\begin{abstract}
OF THE DISSERTATION
A METHODOLOGY TO ESTIMATE TIME VARYING USER RESPONSES TO

TRAVEL TIME AND TRAVEL TIME RELIABILITY IN A ROAD PRICING

ENVIRONMENT

by
\end{abstract}

Patricio Alvarez

Florida International University, 2012

Miami, Florida

Professor Mohammed Hadi, Major Professor

Road pricing has emerged as an effective means of managing road traffic demand while simultaneously raising additional revenues to transportation agencies. Research on the factors that govern travel decisions has shown that user preferences may be a function of the demographic characteristics of the individuals and the perceived trip attributes. However, it is not clear what are the actual trip attributes considered in the travel decision- making process, how these attributes are perceived by travelers, and how the set of trip attributes change as a function of the time of the day or from day to day.

In this study, operational Intelligent Transportation Systems (ITS) archives are mined and the aggregated preferences for a priced system are extracted at a fine time aggregation level for an extended number of days. The resulting information is related to corresponding time-varying trip attributes such as travel time, travel time reliability, charged toll, and other parameters. The time-varying user preferences and trip attributes are linked together by means of a binary choice model (Logit) with a linear utility function on trip attributes. The trip attributes weights in the utility function are then 
dynamically estimated for each time of day by means of an adaptive, limited-memory discrete Kalman filter (ALMF).

The relationship between traveler choices and travel time is assessed using different rules to capture the logic that best represents the traveler perception and the effect of the real-time information on the observed preferences. The impact of travel time reliability on traveler choices is investigated considering its multiple definitions.

It can be concluded based on the results that using the ALMF algorithm allows a robust estimation of time-varying weights in the utility function at fine time aggregation levels. The high correlations among the trip attributes severely constrain the simultaneous estimation of their weights in the utility function. Despite the data limitations, it is found that, the ALMF algorithm can provide stable estimates of the choice parameters for some periods of the day. Finally, it is found that the daily variation of the user sensitivities for different periods of the day resembles a well-defined normal distribution. 


\section{TABLE OF CONTENTS}

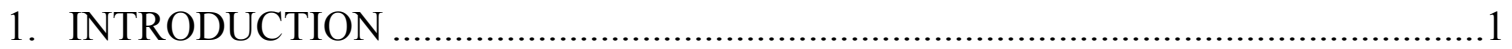

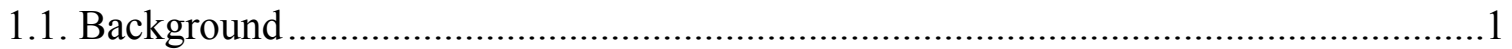

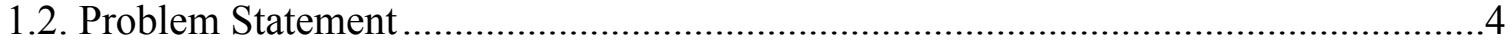

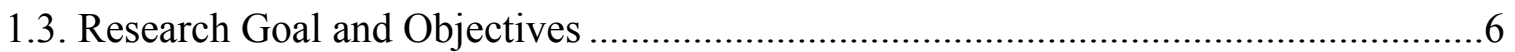

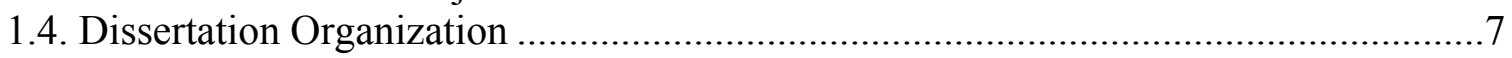

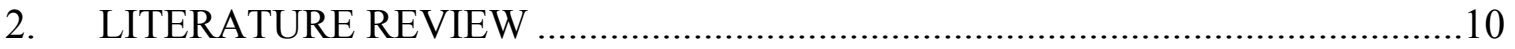

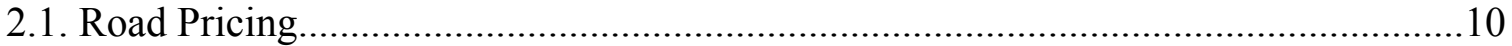

2.1.1. Road Pricing Model for Analysis and Design ..............................................12

2.1.2. Road Pricing and Travelers Heterogeneity .....................................................13

2.1.3. Time Dimension in Road Pricing Models .......................................................14

2.1.4. Models for Road Pricing Impacts Evaluation...............................................15

2.2. User Behavior and Travel Time Reliability .........................................................18

2.2.1. Travel Time Reliability Metrics ………………....................................22

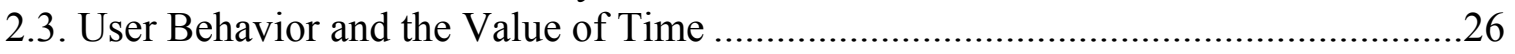

2.3.1. Travel Time Perception and Learning in Transportation Systems .................29

2.3.2. Drivers Perception and Processing of Information.........................................30

2.3.3. Travel Choices and Network Performance .....................................................32

2.3.4. Perception and Integration of Travel Choices and System Performance ........34

2.3.5. Accounting for Integration and Uncertainty in System Performance...............36

2.3.6. A Model for Information Processing and Learning ........................................36

2.4. Intelligent Transportation System (ITS) Data.............................................................

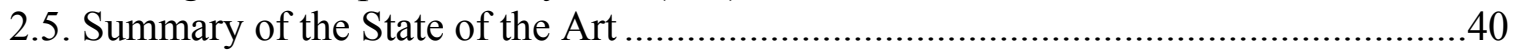

3. PARAMETER ESTIMATION OF DYNAMIC SYSTEMS ....................................42

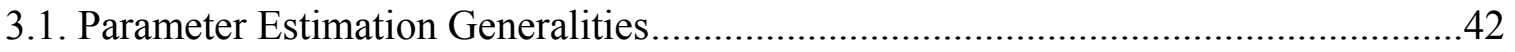

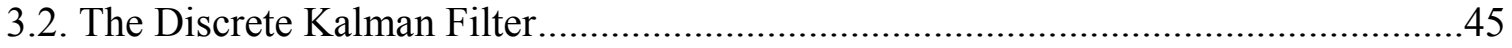

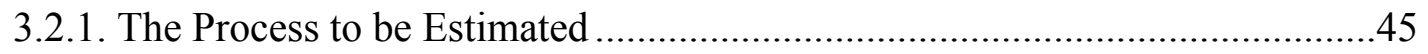

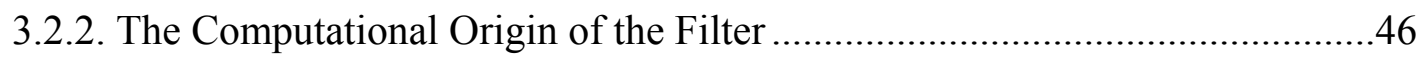

3.2.3. The Discrete Kalman Filter Algorithm .......................................................48

3.3. Adaptive Kalman Filter.....................................................................................

3.3.1. Sequential Estimation with Unknown Noise Statistics...................................51

3.3.2. Empirical Estimation of Measurement Noise Statistics ..................................53

3.3.3. Empirical Estimation of State Noise Statistics ...............................................54

3.3.4. The Kalman Adaptive Memory Limited Filter (AMLF) Algorithm ...............55

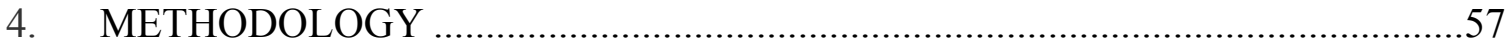

4.1. Learning and Processing Information for a Facility …….......................................58

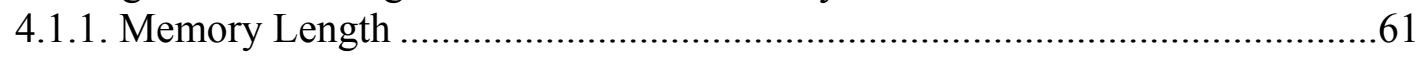

4.1.2. Fading Memory ………………………………...................................63

4.1.3. Real Time Information Impact …………………….................................66 
4.1.4. Gestalt Characteristics of the Travel Time ………….....................................68

4.1.5. Model for Information Processing and Learning Summary ............................70

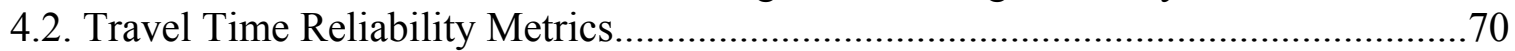

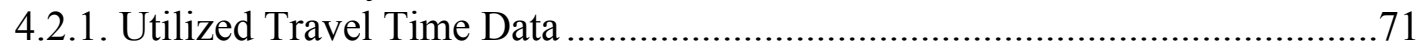

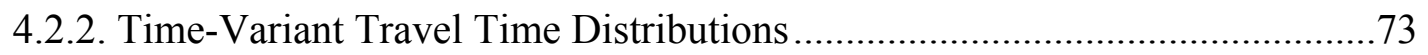

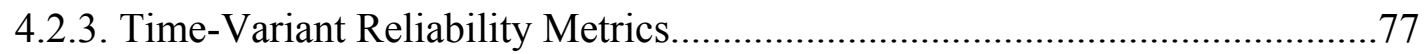

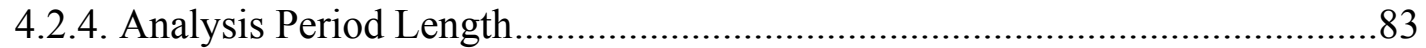

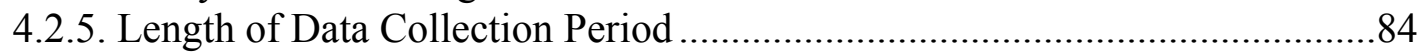

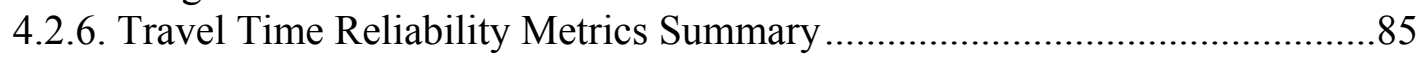

4.3. Adaptive Limited Memory Filter Performance …………...........................................87

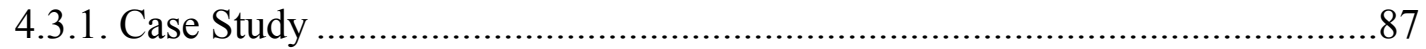

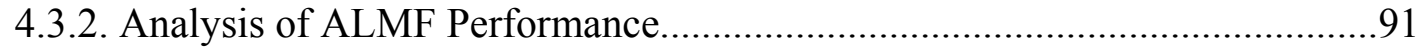

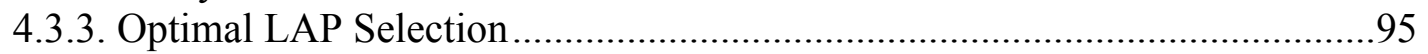

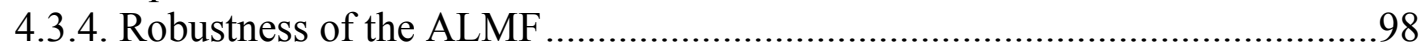

5. TRAVEL TIME AND TRAVEL TIME RELIABILITY SENSITIVIES IN A

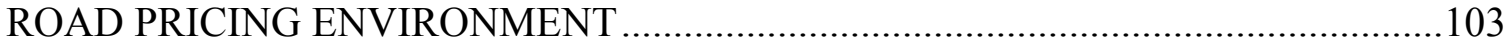

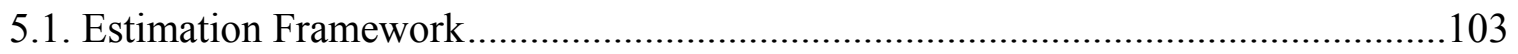

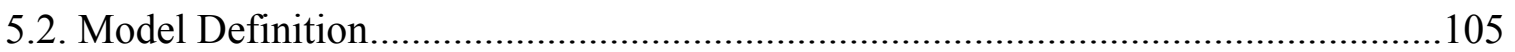

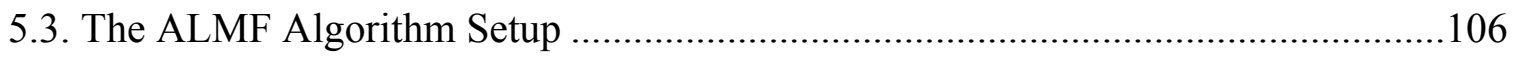

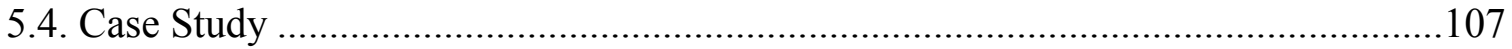

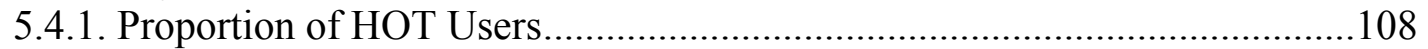

5.4.2. Travel Time

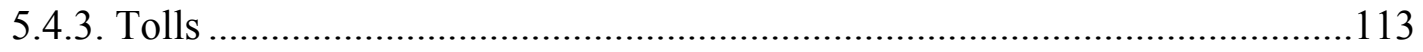

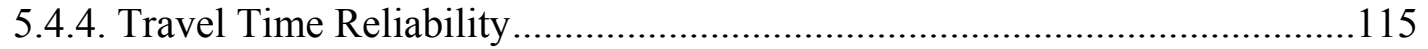

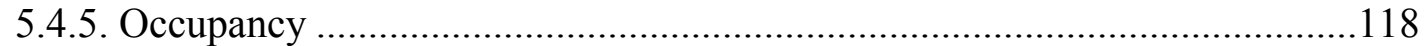

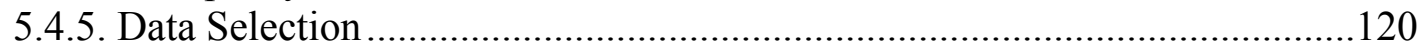

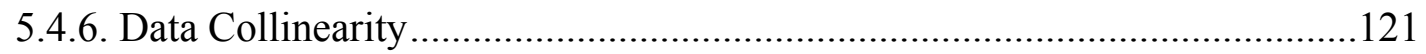

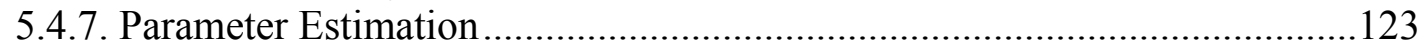

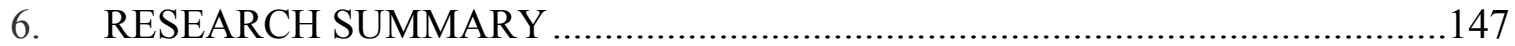

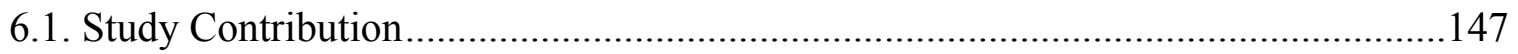

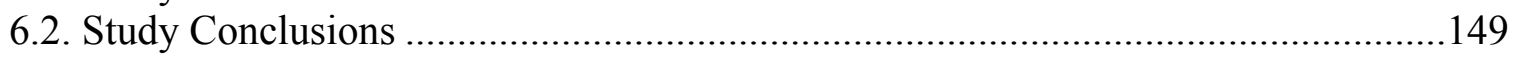

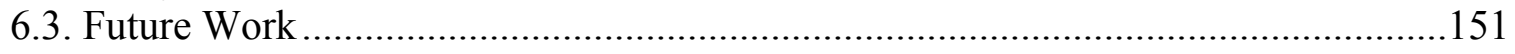

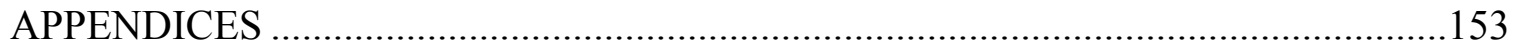

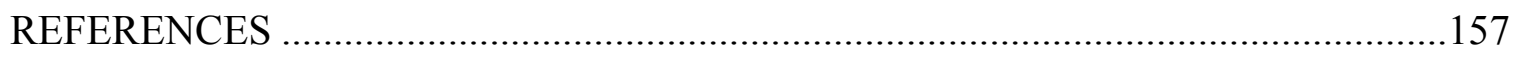

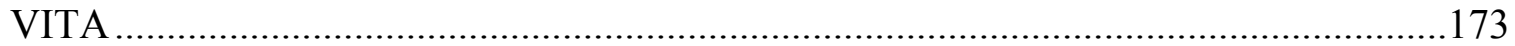




\section{LIST OF TABLES}

TABLE

PAGE

Table 3.1: Discrete Kalman Filter Equations.............................................................. 49

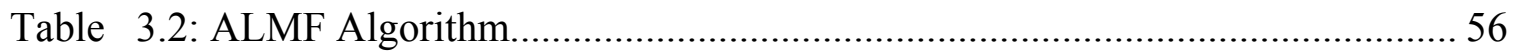

Table 4.1: Data Processing Specifications for Learning and Information Processing ... 61

Table 4.2: Travel Time Reliability Operational Definitions......................................... 78

Table 4.3: Parameter Values Used in ALMF Testing.................................................... 89

Table 4.4: Parameter values used in ALMF Testing …………................................. 92

Table 4.5: Parameter values used in ALMF Testing ……............................................. 99

Table 5.1: Typical Correlation Factors .................................................................... 123

Table 5.2: LAP Values Used on Each Estimation Problem........................................... 127 


\section{LIST OF FIGURES}

FIGURE

PAGE

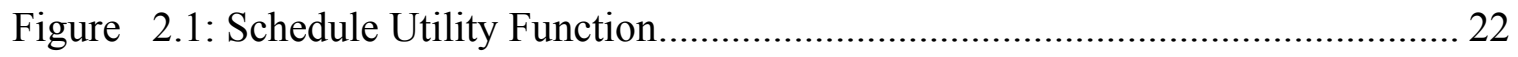

Figure 2.2: Driver Decision and Information Processing Components ........................ 30

Figure 2.3: Drivers' Dynamic Acquisition and Processing of Information................... 32

Figure 3.1: Simplified Block Diagram of the Estimation Procedure Approach ............ 43

Figure 3.2: Measurement Variance Variation along the Day ................................... 51

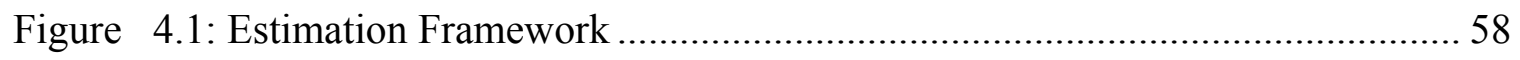

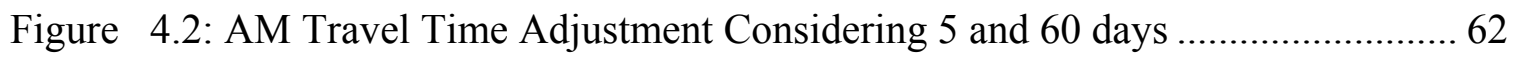

Figure 4.3: PM Travel Time Adjustment Considering 5 and 60 days .......................... 62

Figure 4.4: $R^{2}$ considering different dataset lengths in AM and PM periods.................. 63

Figure 4.5: Exponentially Decreasing Values of $w_{k}$ for Different Values of $b$............ 64

Figure 4.6: AM Travel Time Adjustment Considering $b=1.0$ and $b=1.15 \ldots \ldots \ldots \ldots \ldots \ldots . .65$

Figure 4.7: PM Travel Time Adjustment Considering $b=1.0$ and $b=1.15 \ldots \ldots \ldots \ldots \ldots \ldots . . .65$

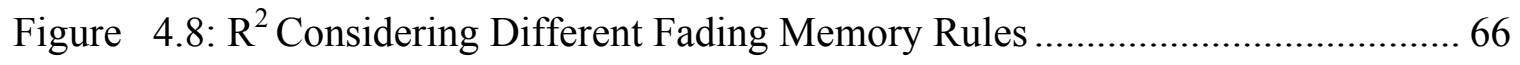

Figure 4.9: AM Travel Time Adjustment Considering $a=0.0$ and $a=1.0 \ldots \ldots \ldots \ldots \ldots \ldots \ldots . \ldots . \ldots . \ldots . \ldots$

Figure 4.10: PM Travel Time Adjustment Considering $a=0.0$ and $a=1.0 \ldots \ldots \ldots \ldots \ldots \ldots . . .67$

Figure 4.11: $R^{2}$ Considering Different Real Time Information Parameter $a \ldots \ldots \ldots \ldots \ldots . . . .68$

Figure 4.12: Relationship Between Proportion of HOT Users and the Gestalt

Characteristics of the Travel Time, 20/60, 10/60, 5/60 and 1/60 respectively ........ 69

Figure 4.13: Travel Time Distribution Shape Parameters ...................................... 77

Figure 4.14: Reliability Metrics Variation with Time-of-Day GP Lanes ..................... 82

Figure 4.15: Reliability Metrics Variation with Time-of-Day HOT Lanes................... 82 
Figure 4.16: Impact of Aggregation Levels on the $80^{\text {th }}$ Percentile PTI Metric 84

Figure 4.17: Effect of Data Collection Period Length .............................................. 85

Figure 4.18: Typical Daily Performance Measures Variation in I-95 .......................... 89

Figure 4.19: Pattern of Variation of $x_{k}$ as Used in the Experiment.............................. 90

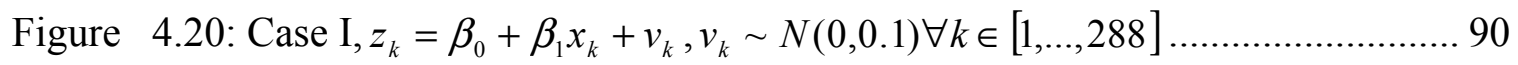

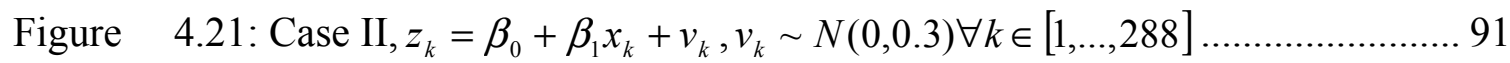

Figure 4.22: Case III, $z_{k}=\beta_{0}+\beta_{1} x_{k}+v_{k}, v_{k} \sim N(0,0.1) \forall k \in[1, \ldots, 144]$, $v_{k} \sim N(0,0.3) \forall k \in[145, \ldots, 288] \ldots$ 91

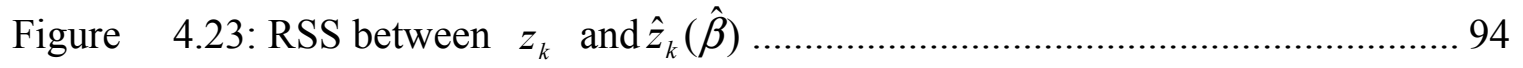

Figure 4.24: Observed and modeled measurement signal using ALMF and KF........ 94

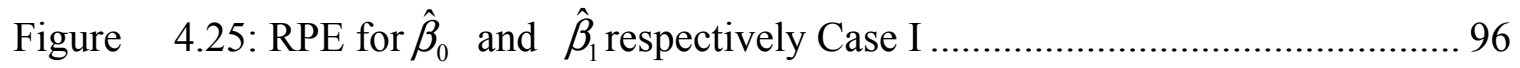

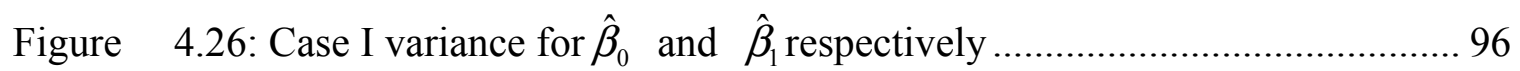

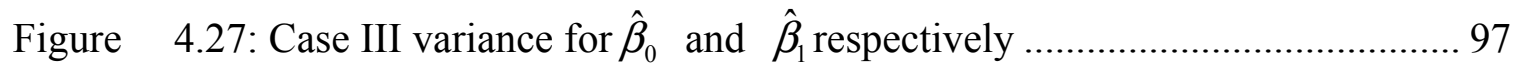

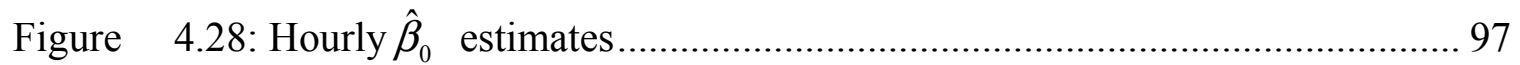

Figure $\quad 4.29$ : Hourly $\hat{\beta}_{1}$ estimates ................................................................... 98

Figure 4.30: ALMF algorithm robustness to $\hat{\beta}_{0}$ and $\hat{\beta}_{1}$ initial values .................... 100

Figure 4.31: Hourly $\hat{\beta}_{0}$ and $\hat{\beta}_{1}$ for different initial values of $\mathrm{P}_{11}$ and $\mathrm{P}_{22} \ldots \ldots \ldots \ldots \ldots . . \ldots 1$

Figure 4.32: Hourly $\hat{\beta}_{0}$ and $\hat{\beta}_{1}$ for different initial values of $q_{1}$ and $q_{2} \ldots \ldots \ldots \ldots \ldots \ldots . . . . . .101$

Figure 4.33: Hourly $\hat{\beta}_{0}$ and $\hat{\beta}_{1}$ for different initial values of $\mathrm{Q}_{11}$ and $Q_{22} \ldots \ldots \ldots \ldots \ldots 101$

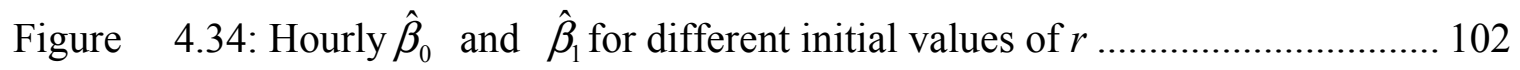




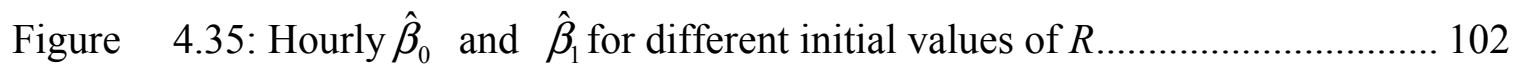

Figure $\quad$ 5.1: Study Area and HOT Layout.......................................................... 108

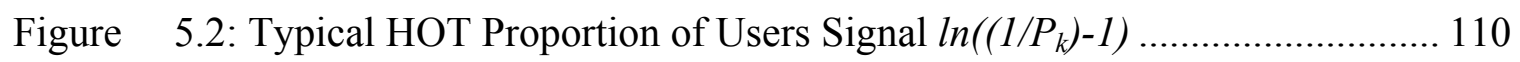

Figure 5.3: Daily Variation of HOT Proportion of Users Signal $\ln \left(\left(1 / P_{k}\right)-1\right) \ldots \ldots \ldots \ldots . .111$

Figure $\quad$ 5.4: Typical GP and HOT Travel Time Difference.................................... 112

Figure 5.5: Travel Time Difference Day to Day Statistics ..................................... 113

Figure 5.6: Typical Toll Values During Weekdays .............................................. 114

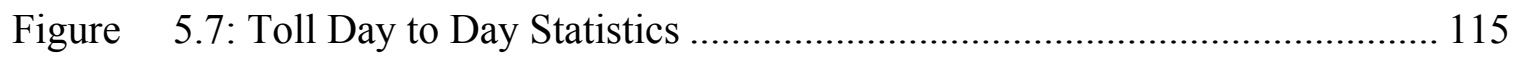

Figure 5.8: Relationship between HOT Users and the Misery Index Difference....... 117

Figure 5.9: Relationship between HOT Users and the Buffer Index Difference........ 117

Figure 5.10: Relationship between HOT Users and the Skew Statistic Difference.... 117

Figure 5.11: Typical Relationship Between HOT Users and the FOT1.1 ................ 118

Figure 5.12: Typical Relationship Between HOT Users and the FOT1.25 .............. 118

Figure 5.13: Typical Occupancy Difference Values During Weekdays.................... 119

Figure 5.14: Occupancy Day to Day Statistics .................................................... 120

Figure 5.15: Typical Relationship between Travel Time and Toll .......................... 121

Figure 5.16: Typical Relationship between Travel Time and PTI $95^{\text {th }}$.................... 122

Figure 5.17: Typical Relationship between Travel Time and PTI $80^{\text {th }} \ldots \ldots \ldots \ldots \ldots \ldots . . . . . . . .122$

Figure 5.18: Typical Relationship between Travel Time and Occupancy................. 122

Figure 5.19: Estimate Variance along the Day Different LAP values...................... 126

Figure 5.20: Estimate Variance Selected Hours for Different LAP values ............... 126

Figure 5.21: AM and PM Regression Between the Observed signal and Selected

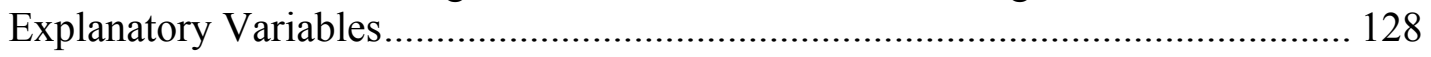


Figure 5.22: Time Varying Values of $-\beta_{0}$ Travel Time ............................................ 130

Figure 5.23: Time Varying Values of $\beta_{1}$ Travel Time ............................................. 130

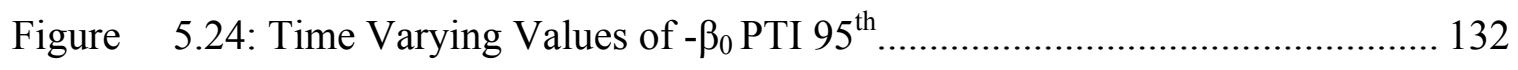

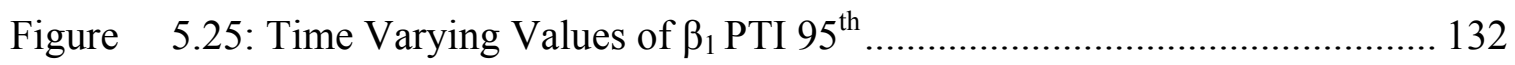

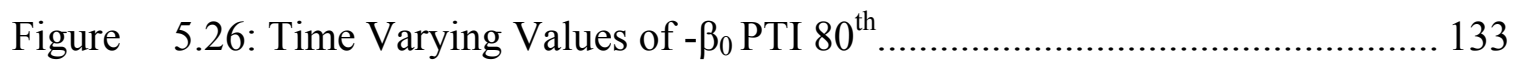

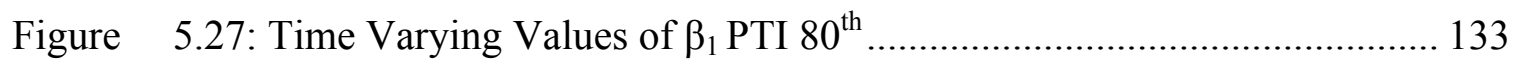

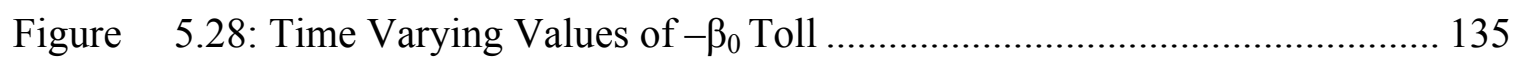

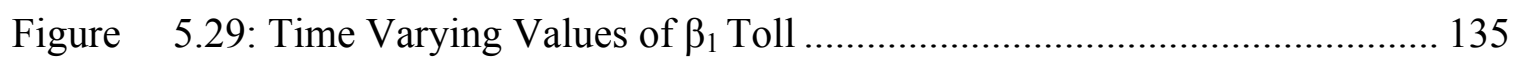

Figure 5.30: Proportion of HOT Users as Function of Toll and Time ....................... 136

Figure 5.31: Time Varying Values of $-\beta_{0}$ Occupancy …………………………....... 137

Figure 5.32: Time Varying Values of $\beta_{1}$ Occupancy …………………................... 137

Figure 5.33: Time Varying Values of $\beta_{1}$ Occupancy ................................................. 138

Figure 5.34: $\beta_{0}$ and $\beta_{1}$ Time Varying Mean, $95^{\text {th }}$ Confidence Interval and Standard Deviation with Travel Time as Explanatory Variable ............................................. 139

Figure 5.35: $\beta_{0}$ and $\beta_{1}$ Time Varying Mean, $95^{\text {th }}$ Confidence Interval and Standard Deviation with Planning Time Index $95^{\text {th }}$ as Explanatory Variable ....................... 139

Figure 5.36: $\beta_{0}$ and $\beta_{1}$ Time Varying Mean, $95^{\text {th }}$ Confidence Interval and Standard Deviation with Planning Time Index $80^{\text {th }}$ as Explanatory Variable

Figure 5.37: $\beta_{0}$ and $\beta_{1}$ Time Varying Mean, $95^{\text {th }}$ Confidence Interval and Standard Deviation with Toll as Explanatory Variable ......................................................... 140

Figure 5.38: $\beta_{0}$ and $\beta_{1}$ Time Varying Mean, $95^{\text {th }}$ Confidence Interval and Standard Deviation with Occupancy as Explanatory Variable....

Figure 5.39: $\beta_{0}$ and $\beta_{1}$ Time Varying Mean, $95^{\text {th }}$ Confidence Interval and Standard Deviation with Travel Time and Toll as Explanatory Variables.....

Figure 5.40: $\beta_{0}$ and $\beta_{1}$ Time Varying Mean, $95^{\text {th }}$ Confidence Interval and Standard Deviation with PTI 95 and Toll as Explanatory Variables 
Figure 5.41: $\beta_{0}$ and $\beta_{1}$ Time Varying Mean, $95^{\text {th }}$ Confidence Interval and Standard Deviation with PTI 80 and Toll as Explanatory Variables

Figure 5.42: $\beta_{1}$ Distribution Between 13:00 and 20:00 when Travel Time is the Explanatory Variable 143

Figure 5.43: $\beta_{1}$ Distribution Between 13:00 and 20:00 for PTI 95 ${ }^{\text {th }} \ldots \ldots \ldots \ldots \ldots \ldots \ldots \ldots . . . .143$

Figure 5.44: $\beta_{1}$ Distribution Between 13:00 and 20:00 for PTI 80 ${ }^{\text {th }} \ldots \ldots \ldots \ldots \ldots \ldots \ldots \ldots \ldots . . . . .144$

Figure 5.45: $\ln \left[\left(1 / P_{k}\right)-1\right]$ as Function of Time for Different Levels of Diversion in the GP lanes 145

Figure 5.46: $\beta_{0}$ and $\beta_{1}$ Time Varying Mean, $95^{\text {th }}$ Confidence Interval and Standard Deviation with Planning Time Index $95^{\text {th }}$ as Explanatory Variable. 146 


\section{CHAPTER 1}

\section{INTRODUCTION}

\subsection{Background}

Quality of life is strongly related to the ability to travel in a timely manner. Every day, millions of travelers around the world make travel related decisions in order to meet their needs. Particularly, American commuters consistently rank mobility among the top three regional policy issues, together with the economy, education, and crime (Knickerbocker, 2000). Economic development and other advances in modern civilization are also accompanied by social and environmental problems. One of the most noticeable problems is traffic congestion, which has become part of daily life for commuters in many urban areas.

Due to the growth in people's mobility, demand for roadways currently exceeds the supply, particularly at the peak hours. This imbalance is causing reduced speeds, increased travel times, reduction in travel time reliability, higher fuel consumption, vehicle wear, safety problems, impacts on the environment, and inconvenience from rescheduling trips or relocating residences and jobs in the long term to avoid congestion.

The enlarging gap between travel demand and infrastructure supply is an ongoing problem that has increased traffic congestion nationwide. In the United States, during the past 25 years, vehicle miles traveled (VMT) has increased by 80 percent while lane capacity has grown by only 2 percent; likewise, roadway congestion in the 75 most congested urban areas annually caused more than 3.5 billion hours of delay (Standard \& Poor's, 2005). 
From the point of view of decision makers, the provision of future mobility must be framed by realistic consideration of available financial resources. Building new roads or increasing capacity of existing roads to alleviate congestion is generally expensive. Moreover, it has been reported that expansion of the transportation system capacity can allow latent demand to consume much of the expected travel time savings (Gullipalli P. 2008). Thus, an attractive alternative to account for budget constraints and growing needs is to improve the efficiency of the transportation system through active traffic and demand management, so that the demand can be accommodated at a lower cost.

Road pricing has emerged as an effective means of both managing road traffic demand and at the same time raising additional revenue. The rationale behind road pricing comes from the observation that people tend to make socially efficient choices when they are faced with all the social benefits and costs of their actions. Road pricing is justified because motorists are unaware of certain costs their behavior imposes on others (Litman, 2011). Under a road pricing strategy, road users are charged a fee that better reflects a measure of the cost of their travel decisions than do existing fees and taxes. Pricing can therefore serve as a public policy tool to help manage demand for a limited resource (TRB, 2005). As in any other pricing system, road pricing allocates road space to those most willing to pay for it, provides guidance in the revenues collected as to where capacity extension is needed, and creates a funding source for repaying the investment. 
A road pricing schema that has been particularly used often in the U.S., is the provision of a fee differentiated service at locations where spare capacity is still available. In the case of high occupancy tolled lanes (HOT), individual drivers can pay a variable price for the privilege of joining high occupancy vehicles (HOV) in the restricted lanes.

In terms of user responses, road pricing has the potential to affect many dimensions of travel behavior. In the short term, the primary impacts of road pricing relate to route choice, departure time-of-day choice (peak spreading), and the mode of transportation choice. For global area pricing forms (typically, drivers paying a fee to cross a cordon and enter a congested central city area during business hours), the entire daily activity pattern of individuals can be changed with important implications for the number and chaining of trips over the entire course of the day (Vovsha et al., 2005).

Traditionally, user behavior related to road pricing has been estimated using individual data collected by means of either stated preferences surveys (SP), reveled preferences surveys (RP), simulation, or by means of a mix of the approaches. However, the survey approach is expensive, particularly for large systems. In addition, the data collected using such methods are normally representative of one or a few days and may not capture fine temporal variations in user preferences and other features like user decisions under non-recurrent congestion conditions (incidents, weather, etc.). With respect to the alternative survey methods, SP surveys tend to have serious limitations, due to the fact that their answers to the surveys may not reflect the individual's actual behavior, but just an intention. In case of using simulation techniques, the main criticism points to the fact that neither the transportation system is a real system nor the users are actually commuting. 
Intelligent Transportation Systems (ITS) refers to transportation systems which apply emerging hard and soft information systems technologies to address and alleviate transportation congestion. The data gathered by such systems are processed and turned into valuable information that is used at the policy, strategic, and tactical levels of the decision process. As ITS data archives become more widely available, the utilization of such archives for estimation of road pricing user behavior can be an attractive option. This utilization can provide a significantly lower cost and a cost-efficient data collection method compared to traditional methods. The additional details provided by the ITS data, both in time and space resolutions, allow better representations of real-world environments in transportation models. For example, the use of archived ITS data allows the consideration of drivers behavior for a number of traffic conditions; including, special events, accidents, work zones, weather events, and incident management strategies. (Alvarez et al., 2010)

\subsection{Problem Statement}

When considering the implementation of traffic and/or demand management strategies, estimating the benefits of implementing road pricing policies is of natural interest. Assessing road pricing strategies requires the use of models under a number of assumptions about the mechanisms used to decide the several dimensions of their travel behavior. In this regard, road pricing adds significant complexity to all choice dimensions since it requires a detailed consideration of the travelers' willingness to pay, as well as detailed description of their sensitivities to the level of service provided by the system; such as, travel time, travel time reliability, safety, comfort, and others. 
These user behavioral factors have been the subject of decades of substantial theoretical and empirical research. If we consider travel time alone, research has indicated that income, trip purpose, time of day, and method of payment highly determine how travel time is related to user behavior. However, because the use of surveys to estimate the impact of these behavioral factors, the estimated values of these impacts can only represent average conditions during a period of time, and thus can be used to assess road pricing strategies only for similar levels of aggregation in the analysis. However, more fine-grained analysis models using the parameters calibrated as described above may be not sufficiently sensitive to the variability of the traffic conditions in real time or between days. These deviations from the average conditions may be the result of variations in the system; such as, weather, surface conditions, incidents, etc. Such variations may cause traffic conditions to differ significantly from the average scenario evaluated by the surveys. In addition, factors such as the impact of provided travel information and the actual pricing strategy may not be sufficiently captured. Thus, the predictive power of the models used to assess the road pricing operation may be significantly reduced.

In addition to travel time, there is a growing body of empirical evidence that travelers value reliability as an important factor in their trip making decisions. Travel time reliability becomes especially important in dynamic pricing applications, where tolls are adjusted based on traffic congestion level in order to maintain a specified level of service. In the case of the HOT lanes, users may experience only a small reduction in their average travel time over non-toll lanes, but enjoy a substantial reduction in their travel time day-to-day variability. This increased reliability can be critical for travelers with 
rigid schedule requirements (e.g., day-care pickups, workers on time-clocks, or airline passengers) and is not necessarily correlated with the traveler's perception of the travel time. Despite the potential importance of reliability to road pricing, there are few, if any, examples of travel time reliability assessment for HOT applications considering fine time resolution levels.

Finally, regarding ITS data, prior to the widespread deployment of traffic detector equipment and the establishment of traffic management centers in major urban areas, there was limited data available to measure hourly and day-to-day changes in the transportation system. Recent expansion of ITS systems and the archiving of this data allow the exploration of pricing impacts and the effects of external factors such as weather and incidents on user choices. In this regard, ITS data can be used to study the variations of the user behavior and choices made in a priced system, under different traffic and environmental conditions at the level of temporal aggregation desired. ITS data can be processed using alternative procedures in order to reveal the traveler's logic that best describe his decision making.

\subsection{Research Goal and Objectives}

The goal of this dissertation is to develop a methodology to estimate the user sensitivities to travel time and travel time reliability in a system managed using road pricing strategies. The methodology focus is on the estimation of the user sensitivities to travel time and travel time reliability with special attention on the effect of the time-of-the-day and day-to-day traffic variations. To achieve this goal, the following objectives must be fulfilled: 
1) Review the existing road pricing schemas, models, and their considerations of user behaviors; the existing methodologies to estimate the user choice sensitivities to travel time and travel time reliability; and the available models to assess the user perception and learning of travel time.

2) Develop an effective methodology to estimate dynamic aggregated models of user responses to road pricing utilizing ITS data archives as primary data source.

3) Estimate the time varying distribution of sensitivities to travel time and travel time reliability in a priced corridor.

\subsection{Dissertation Organization}

This dissertation is organized into six chapters. Chapter 1 introduces the research background, states the problem to be solved, and sets the goal and objectives to be achieved.

Chapter 2 presents an extensive literature review regarding road pricing application strategies and schemas, modeling approaches to road pricing, consideration of travelers behavior in road pricing applications, treatment of time in road pricing models, and road pricing models to assess the impact on the traffic patterns and the environment. Also in the chapter is a description of the relationship between the travel time and the travelers' behavior and a review of existing approaches to account for traveler's travel time perception and learning. Similarly, a description of the relationship between of the travel time reliability and travelers' behavior, and a summary of the different travel time reliability metrics proposed are provided. In addition, a section describing the advances 
and possibilities of ITS data warehousing for transportation models estimation closes the chapter.

Chapter 3 discusses the basic components of the methodology to estimate traveler's behavior parameters. First, travelers' characteristics regarding perception and integration of travel time information are explored and a model to represent such processes is proposed. Also this chapter discusses in depth the variations of travel time by time of day at fine temporal aggregation levels, the sensitivity of various reliability metrics to these variations, the effect of the aggregation level choices on the calculated metrics, and the amount of data required to estimate stable values of the reliability metrics.

Chapter 4 describes the development of an adaptive optimal estimation algorithm based on the discrete Kalman filter. The algorithm is developed in order to minimize the amount of a priori information required to perform an optimal estimation of the parameters of interest (ALMF). The robustness of the estimation algorithm is tested and discussed. Finally recommendations are proposed for further application of the ALMF to real world problems.

Chapter 5 is devoted to the estimation of the behavioral parameters in a corridor managed using road pricing techniques using the ALMF algorithm developed as described in Chapter 3. The travelers' responses are explored by mining ITS detector databases archived in traffic management centers (TMC) operational files. Those responses are associated with changes in transportation system performance measures (travel time, travel time reliability, toll) by utilizing a Logit model with unknown parameters that can be estimated using the ALMF algorithm. Optimal ALMF tuning and 
setup are explored, and the suitability of different combinations of explanatory variables is addressed by means of correlation analysis. Finally, travelers' time-of-the-day behavior and travelers' day-to-day behavior are investigated based on the results of applying the ALMF algorithm to a corridor in South Florida.

Chapter 6 summarizes the main contributions, draws conclusions, and recommends issues worth to consider for future research. 


\section{CHAPTER 2}

\section{LITERATURE REVIEW}

This section provides a detailed review of the state-of-the-art of road pricing manifestations, models developed for selection of road pricing parameters, road pricing as affected by travelers heterogeneity, the time dimension consideration in road pricing models, models for road pricing impacts evaluation, travel time reliability impact on user behavior, and user behavior as affected by the travel time. Also, a description of the relationship between the travel time and the traveler's behavior and a review of existing approaches to account for traveler's travel time perception and learning are included. In addition, a review of previously investigated relationships between the travel time reliability and travelers' behavior, and a summary of the different travel time reliability

metrics are provided. Further, a section describing the advances and possibilities of utilizing ITS data warehousing for transportation models estimation closes the chapter.

\subsection{Road Pricing}

Road pricing has received significant consideration in the scientific literature and the results from several surveys of literature previously conducted (De Palma et al., 2006; Lindsey, 2005; Mcdonald, 2004; Morrison, 1986; Newbery, 1990; Parry et al., 2007; Sharp et al., 1986). This consideration is also demonstrated by the availability of books, collections of papers, and conference proceedings on the subject (Bekiaris et al., 2004; Button et al., 1998; Jensen-Butler et al., 2008, Lawphongpanich et al., 2006; Levinson, 2002; Pickfor et al., 2006; Roth, 1996; Roth (Ed.), 2006; Small et al., 2007; Viegas (Ed.), 2005; Yang et al., 2005). 
A variety of road toll pricing schemes, including congestion charging, toll cordons, and toll lanes, have been applied in practice worldwide (De Palma et al., 2007; TRB, 2005). In addition, the potential deployment of a number of road pricing schemes has been studied with regard to their feasibility, efficiency, revenue generation, and acceptability in various urban areas around the world (De Palma et al., 2006; Gibbons et al., 2002; Ison, 1998; Proost et al., 2002; Santos et al., 2001); Asian cities and countries, like Hong Kong (Dawson et al., 1986; Harrison et al., 1986; Hau, 1990; Hills, 1984; Pretty, 1988), Seoul (Kim et al., 2005) and Bangkok (Kunchornrat et al., 2008), Japan (Matsuda et al., 2005, Yamamoto et al., 2000, Ying et al., 2007); and developing countries in South East Asia, Africa and Central America (Anderson, 1989; Armstrong-Wright, 1986; Churchill et al., 1972; Gakenheimer,1999; Johansen,1989).

Depending on the network management strategy, and the planning and policy objectives and constraints associated with the road provider(s), the road pricing design process can aim at:

- Managing travel demand, congestion, and possibly other external (e.g. environmental) costs of road usage,

- Raising revenues for funding transport investment, operations, and maintenance costs, and

- Combining revenue generation with travel demand and congestion management. 
Practical experience from the implementation of congestion pricing schemes to tackle road traffic congestion has already been gained in several European cities, including London (Goodwin, 2004; Leape, 2006; Richards, 2006; Santos et al., 2006), and Stockholm (Armelius et al., 2006; Eliasson, et al., 2006; Mattsson, 2008).

Other applications of road pricing include the (statically or dynamically valued) high occupancy toll (HOT) lanes in the United States to allow users of low occupancy or single-occupancy vehicles to join the express lanes if they pay the corresponding toll (Lam, 2004; Weinstein et al., 2006). Another example of these applications is the toll rings in Norway (Larsen et al., 2001; Odeck et al., 2002). Other pricing schemes have also been considered in the United States, like truck-only toll lanes (Holguín-Veras et al. 2003); and mechanisms to address equity issues, such as credit-based congestion pricing (Kockelman et al., 2005), where revenues are redistributed uniformly among users as a sort of driving "allowance"; Fast and Intertwined Regular (FAIR) lanes (De Corla-Souza, 2004), where toll credits is provided to users of adjacent lanes in multi-lane facilities; and the integrated multimodal strategy of FAST Miles (DeCorla-Souza, 2006). Other proposed strategies include vehicle mileage-based pricing strategies (Oh et al., 2007) and combinations with pay-as-you-drive insurance or car-sharing, which aim at converting the fixed charges for driving to variable charges (DeCorla-Souza, 2002).

\subsubsection{Road Pricing Model for Analysis and Design}

The road pricing design problem can be modeled as a bi-level optimization problem or a mathematical program with equilibrium constraints. In general, the bi-level optimization structure of the road pricing can be expressed through the following relationships: 


$$
\begin{array}{ll}
\max _{p, q} F(p, q(p)) & \\
\text { s.t. } & G(p, q(p)) \leq 0 \\
& \min _{q} f(p, q) \\
& \text { s.t. } g(p, q) \leq 0
\end{array}
$$

At upper-level problem, $F$ denotes the objective function of the decision maker, so that some measure of user or social surplus is maximized or some other objective is achieved. The function $G$ represents the constraint set of the decision vector, which may involve a range of different requirements or restrictions. At the lower level problem $f$ represents the response function of users with respect to tolls and other system attributes like travel time and travel time reliability, towards achieving an equilibrium flow distribution $q(p)$ subject to a set of constraints denoted by $g$. The equilibrium state is traditionally expressed by a flow pattern in which no traveler could decrease the actual travel cost by unilaterally changing the route (Wardrop, 1952). A more realistic extension of this principle refers to stochastic user equilibrium, based on which travelers can change their perceived travel cost by unilaterally changing the route (Daganzo et al., 1977). Extensions of the later principle, allows accounting for variations in the perceived travel costs and uncertainty in the route choice (Smith et al., 1994; Yang, 1999).

\subsubsection{Road Pricing and Travelers Heterogeneity}

A number of modeling advances have been developed to allow considering the impacts of user attributes (income, household size, etc.) and vehicle attributes (type, occupancy) on the response to selected pricing schemas. The distinction of these sources of heterogeneity can offer a more realistic representation of travel responses due to varying 
tradeoffs between toll rate, travel time, and other relevant attributes. The assumption of heterogeneous user groups and the associated multi-class assignment procedures have been considered for deterministic user equilibrium (Arnott, 1992; Bellei et al., 2002; Dafermos, 1973; Mayet et al.,1999; Yin et al., 2004; Zhang, 2008) and to a lesser degree with stochastic user equilibrium conditions (Ying, 2005; Zhao et al. 2006).

With the exception of a few studies which dealt with the heterogeneity of the value of travel time (Dimitriou, 2009) and diversified travelers' cost structures (Xin et al., 2007), the current design of dynamic congestion pricing schemes generally does not adequately consider the effects of various attributes of vehicles and users for the imposition of discriminatory tolls.

\subsubsection{Time Dimension in Road Pricing Models}

Several studies have recognized the need for capturing the peaked nature of travel demand and the dynamic nature of traffic flow in the evaluation of different pricing schemes. From a user point of view, acceptance of congestion pricing may involve within-day traffic dynamics, day to day network traffic dynamics, or both together.

Models that consider the impacts of within-day dynamics on user acceptance allow assessing the impacts of time varying charges on different travel choices (departure time, mode, route, and destination) of users. This consideration has been used as part of simplified models which estimate scheduled delay costs (generalized cost considers explicitly the cost of arriving early or late) and account for spatial queue impacts (Arnott, et al., 1993; Braid, 1989; De Palma et al., 2005; Kuwahara, 2007; Laih, 1994; Levinson, 2004; Mun, 1999). More recently Peeta (2001) and Szeto et al. (2006) represented the 
interaction between traffic pattern and congestion charges, using simulation-based dynamic traffic assignment (DTA) considering both the time and spatial dimensions of traffic flow, capturing the queue formation, dissipation, and spillback phenomena; hence, properly allowing the representation of the interaction between the traffic and dynamic congestion charges.

In addition to the above, the impact of day-to-day dynamics on user acceptance of pricing is also important. The dynamics of the behavioral adjustments of tolled road users can be regarded as the combined result of their experience and the information acquisition, and learning processes taking place in both the day-to-day and within-day timescales. In this direction, (De Palma et al., 2005) studied the impact of time-varying congestion tolls on the performance of a road network using a dynamic equilibrium simulator, where a day-to-day adjustment process with exponential learning by drivers governs changes in mode choice, departure time and route choice, and guides the system towards a stationary state.

\subsubsection{Models for Road Pricing Impacts Evaluation}

Most of the modeled impacts of road network pricing schemes concern short term changes in the travel behavior of users (trip-making, departure time, route, and mode choice). The study of other implications of road pricing has focused on transport network performance measures, including improvements in travel speed or travel time and its reliability (Chan et al., 2005; Hensher et al., 2008; Supernak et al., 2003), fossil fuel energy consumption (Lim, 1997; Luo et al., 2007), safety, traffic noise, and air pollution and climate change (Beevers et al., 2005; Chin, 1996; Joumard et al., 1996; Khazzoom et 
al., 1991; Luo et al., 2007; Mitchell, 2005; Noland et al., 2008; Otterström, 1995; Proost et al., 2001; Schuitema et al.,2007).

The modeling frameworks used for the evaluation of road pricing schemes from the traveler's perspective can be assessed based on revealed and/or stated preference surveys, and has included:

- Linear, log-linear, and translog econometric models employing micro-data and, possibly, inequality (Gini, Theil's index) measures (Franklin, 2006) and nonparametric methods (Franklin, 2008) to evaluate differential effects on different population segments;

- Discrete choice models, like those based on count data (e.g., Poisson and negative binomial) regression techniques (Peeta et al., 2001), binomial and multinomial logit (Álvarez et al., 2007; Burris et al., 2002; Hess et al., 2008, Yamamoto et al., 2000), nested logit (Erhardt et al., 2003; Jovicic et al., 2003), conditional logit (Washbrook et al., 2006), rank-ordered Logit (Calfee et al., 1998), ordered-response Probit (Golob, 2001), mixed Logit (Brownstone et al., 2005; Nielsen, 2004; Small et al., 2001; Small et al.,2006) and Generalized Extreme Value (GEV) with correlated random utility error terms (Zhao et al., 2008);

- Microscopic or mesoscopic network models for simulating traffic and user responses to road pricing and other management and control measures (Al-Deek et al., 2007; De Palma et al., 2005; Lee et al., 2007; Murray et al., 2001), which may be integrated with discrete choice models; and 
- Agent-based computational models of travelers' behavior and interactions (Basso et al., 2008a, Holguín-Veras, 2008; Markose et al., 2007; Link, 2008).

Consideration of the road-pricing-related choices in different steps of the trip decision process (such as trip generation, distribution, modal split, and assignment) should be based on the proper modeling of road pricing as related to these steps. To summarize the generalized modeling constructs that are used for road pricing assessment, the following three basic approaches stand out:

- Application of traffic assignment model with generalized impedance functions that incorporates tolls by means of value of time (VOT) estimates, as well as additional delays associated with toll collection. This is the simplest approach that does not require the development, estimation, and application of choice models. Only VOT estimation is necessary. However, there are several strong limitations of this approach, such as ignoring the tolled-off (diverted) travelers who may change mode, destination, time of day, car occupancy, etc., as a result of imposing a toll.

- Application of a binary choice model that considers a choice of toll road versus non-toll options in combination with network equilibrium assignment that uses correspondent networks (with and without toll facility) to ensure travel time saving for those who chose to pay a toll. Two versions of this approach can be identified: 1) treatment of the travelers not willing to pay as toll not users of the facility in the assignment, and 2) treatment of the travelers as a proportion diverted to the tolled facility. In the last case, a binary choice model essentially works as a diversion curve. 
- Modeling toll-road options as an additional component in the travel demand hierarchy of choices fully accounting for travel behavior across all relevant dimensions. The relevant dimensions that are closely intertwined with toll-road choice include mode, car occupancy, and time-of-day. There can be a potential impact on destination and trip-frequency choice as well; however these dimensions are considered less obvious and of second-order importance in practical terms.

\subsection{User Behavior and Travel Time Reliability}

The important issue of travel time reliability may be separately introduced into the analysis of dynamic congestion pricing schemes from two different points of view:

- That of travelers, in terms of a path reliability component in their utility function (Dimitriou, 2009; Sauri et al., 2008; Small et al., 2006), and

- That of road providers, where the optimal charge or optimal links for charging are sought to optimize network travel time reliability, subject to the responses of users.

In the latter case, the road providers may consider stochastic network characteristics (link capacities) and fluctuating elastic demand from day-to-day (Li et al., 2007).

The behavioral response of travelers to travel time reliability has been investigated in a number of studies. In one study, (Abdel-Aty et al., 1997) two stated preference techniques were used (a computer aided telephone interview and a mail-back survey) in order to investigate the effect of travel time reliability and traffic information on commuters. The analysis of the survey data was performed utilizing binary Logit 
models including variables such as the travel time standard deviation, travel time mean, and driver gender. They found that commuters consider reliability characteristics in their route choice preference, and pay attention to travel information enough to be influenced in some scenarios to deviate from their usual routes. Another finding was that male drivers tend to choose the uncertain route more than female drivers.

In another study (Jackson et al., 1981), a survey was administered to Stanford University employees. The survey consisted of paired comparison questions of hypothetical route alternatives. They found that some commuters prefer the more reliable route, even if the expected travel time is higher in comparison to other routes with shorter expected travel time but higher uncertainty.

Other more recent studies by Small (Small et al., 2005; Small et al., 2006) utilized data collected on California State Route 91 (CA-91) in the morning period. The first study (Small et al., 2005) focused solely on formulating a lane choice model (using mixed logit) by combining the RP and SP data. The results of the model indicated that the impacts of travel time and travel time reliability were significant, and that the heterogeneity in these factors is significant as well. In contrast, the second study (Small et al., 2006) models considered not only lane choice, but also vehicle occupancy and transponder acquisition.

The limitations of the previous empirical studies are mostly related to their observational methodology. In the cases of Abdel-Aty et al. (1997) and Jackson and Jucker (1981), the observed route preferences of the subjects, as described earlier, are obtained by stated preference (SP) techniques; they consisted of hypothetical routes with distinct attributes (e.g. travel time). For this reason, the validity of the observed 
preferences may be affected by the lack of realism, and the subject's understanding of the abstract situations. On the other hand, Small et al. (2005 and 2006) collected both RP (actual preferences of subject's lane choice) and SP (hypothetical scenarios to examine subject's lane choice) observations, and consequently enriched their statistical model by pooling both types of data. However, the nature of the survey methods employed didn't allow for some of the variables to be measured during each of the subject's trips.

Other data collection techniques such as equipping the subject's vehicles with Global Positioning System (GPS) devices would have avoided the difficulties mentioned above, and possibly extend the lane choice model into a route choice model by considering arterials near the subjects. For example Li et al. (2004) presented an inspection of the travel time variability in commute trips, and its effects on departure time and route choice, including cases with trip chaining. Li et al. (2005) presented an analysis of the attributes determining whether to choose one or more routes in the morning commute. Zhang (Zhang et al., 2008) presented an estimation of the value of information for travelers, and a comparison of the impact of information with other variables such as travel time, distance, aesthetics, etc.

To include reliability measures in the traveler's utility function, there are basically two main approaches. The first, referred to as the mean-variance approach, assumes that individuals have preferences against the travel time uncertainty per se. The expected utility of an individual can be written (ignoring monetary cost terms) as follows.

$$
U=\eta \mu+\rho \sigma
$$


where $\mu$ is the expected travel time and $\sigma$ is the standard deviation of travel time, while $\eta$ and $\rho$ are sensitivity parameters. Some authors have replaced the standard deviation with another measure of scale, such as an inter-percentile range (Small et al., 2005).

In the second approach, the scheduling approach, the individual holds preferences for timing of activities. Utility is defined directly from outcomes; i.e., being early or late. Travel time variability affects an individual's utility to the extent that the arrival time at an activity (destination) is affected. The individual holds preferences for being early or late, compared to a preferred arrival time (PAT), as discussed by Small et al. (1982) and Noland and Small (1995). Normalizing an individual's PAT to be at time zero, let $-D$ be the departure time. Hence, earlier departure corresponds to a larger D. Let $T$ be the stochastic travel time. In addition, $\alpha, \beta$ and, $\gamma$ are the parameters of the model. Then, in the scheduling approach, the user utility is given by:

$$
U(D, T)=\alpha T+\beta(T-D)^{-}+\gamma(T-D)^{+}+\theta 1_{T>D}
$$

where $(T-D)^{-}$is schedule delay early, $(T-D)^{+}$is schedule delay late. The term $\theta \neq 0$ allows for a discontinuous penalty for lateness.

Ignoring the first term of the disutility (disutility due to travel time), the remaining contributions to utility are shown in Figure 2.1. At the earliest departure time considered, the traveler arrives too early, and suffers disutility as a result of non-zero values of $(T-D)^{-}$. As the arrival time increases, this disutility declines to a point where the traveler arrives exactly at his preferred arrival time. Immediately thereafter, the traveler will arrive late, and the disutility jumps up to the value of $\theta$. As the value of the arrival time increases, $(T-D)^{+}$contributes increasingly to disutility. 


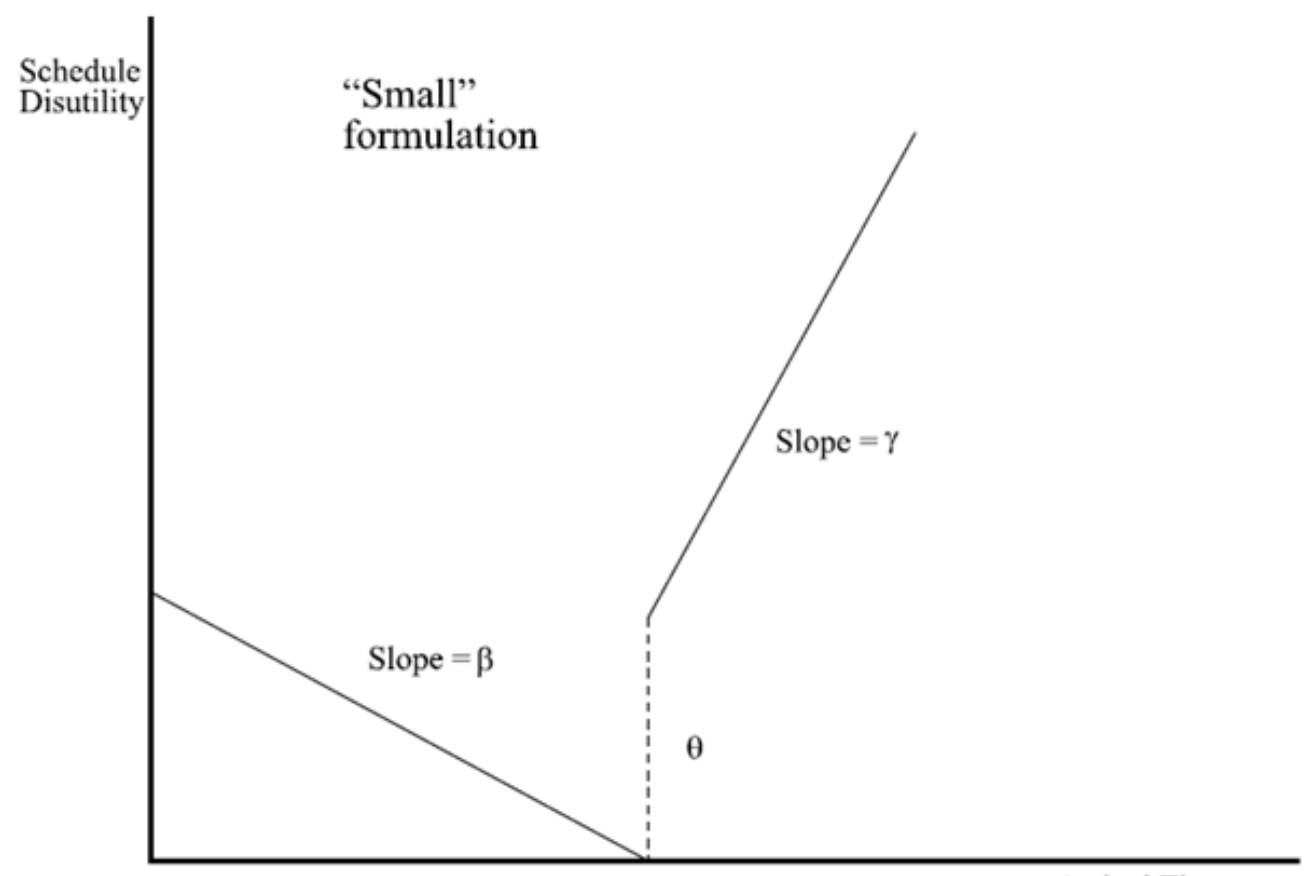

Arrival Time

Figure 2.1: Schedule Utility Function

\subsubsection{Travel Time Reliability Metrics}

As discussed above, travel time reliability is considered an important component of the performance of transportation systems and of travelers' perceptions of this performance. The increased recognition of the importance of travel time reliability is reflected by changes to traditional monitoring programs. For example, in a report published in July 2005, the National Operations Coalition (NTOC) initiative selected the Buffer Index (BI), a travel time reliability measure, as one of few good measures for transportation operations agencies to use for internal management, external communications, and comparative assessments (NOTC, 2005). Public agencies from around the United States are now using travel time reliability metrics as key performance measures to monitor their system operations (GDOT, 2011; FDOT, 2008; SCAG, 2005; WSDOT, 2011). Recognizing the critical need for researching travel time reliability, the Second Strategic 
Highway Research Program (SHRP2, 2011) has specified travel time reliability as one of the four main research areas of the program and has funded extensive research activities in this area.

Travel time reliability measures the level of consistency of travel conditions over time. A unifying reliability definition can be found in final report of the SHRP2 LO3 project (Cambridge Systematics, 2010) where reliability is defined as "the level of consistency in travel conditions over time and is measured by describing the distribution of travel times that occur over a substantial period of time..." The different approaches to defining reliability have led to recommending several metrics for use. These metrics may not necessarily produce consistent assessments of reliability among themselves, since they define travel time consistency in different manners.

Tu (Tu et al., 2007), classified reliability metrics into: statistical range methods, buffer time methods, tardy trip measures, probabilistic measures, and skew-width methods. Lomax (Lomax et al., 2003), discussed the development of reliability measures and the factors to consider before selecting a measure. They concluded that the metrics that are most promising are the Percent of Variation, Misery Index and the BI. This conclusion was based on five factors including the compatibility with multimodal analyses, ability to measure urban and rural travel conditions, consideration of the effects of trip length and time, ability to serve several audiences, and applicability to different area sizes. In a later work, Van Lint and Zulen (Van Lint et al., 2005) noted that the BI and the Misery Index may not be appropriate because of the underlying skewed travel time distribution. They concluded that most of currently utilized reliability metrics should be used and 
interpreted with some reservations.

The common assumption about the normality in travel time distribution was investigated by Rakha (Rakha et al., 2007). The study concluded that the normality assumption is not supported by the observed data. Instead they proposed that a log-normal distribution can describe better the travel time during uncongested conditions. Similarly, during the congested hours a mixed or a bimodal distribution fits better the observed travel time distribution. Additionally, Rakha pointed out that the rate at which the mean changes during the congested hours can be faster than the rate of change of the standard deviation, thus the coefficient of variation may decrease when actually trips are becoming more unreliable.

$\mathrm{Pu}(\mathrm{Pu}, 2010)$, examined analytically a number of reliability metrics assuming a lognormal distribution of travel time with a constant median, while varying the variability and skewness of the travel time distribution. He concluded that the coefficient of variation is a good proxy for a range of reliability metrics and suggested that the use of the $\mathrm{BI}$ is not always appropriate unless $\mathrm{BI}$ is computed based on the median rather than the mean. The latter is because in heavily skewed travel time distributions the use of the mean may underestimate the travel time unreliability. The same author in a different study (Pu et al., 2010) pointed out that some reliability metrics may be inconsistent in their depictions of reliability, such as is the case of the BI that remains constant for different values of the coefficient of variation. As discussed later in this paper, fixing the median while varying the standard deviation and skewness as is done in the Pu study may not reflect real-world conditions, in which the parameters of travel time distributions vary by time of day and may be correlated with each other. 
The SHRP 2 LO3 project (Cambridge Systematics, 2010) examined a set of six reliability metrics to determine their sensitivities to different types of freeway improvements. The utilized metrics were the BI, On-Time Performance, $95^{\text {th }}$ Planning Time Index, $80^{\text {th }}$ Percentile Planning Time Index, Skew Statistic and Misery Index. Based on empirical tests, it was found that all metrics were sensitive to the effects of improvements. However, it was noticed that the 95th percentile travel time or TTI may be too extreme a value to be influenced significantly by operations strategies and that the 80th percentile was more sensitive to these improvements. Another aspect, related to the amount of data required to assess systems reliability was tested, concluding that an absolute minimum of six months of data is required to establish reliability within a small error rate, in areas where winter weather is not a major factor. However, a full year of data is preferred.

Even though reliability metrics have been explored and compared as discussed above, most of the work in the literature have focused on using these measures for relatively coarse levels of aggregation of travel time data, for example for the whole peak periods. Such uses imply that the parameters of the travel time distributions are assumed to remain the same for the whole period of analysis. This may not be sufficient for advanced management strategies such as for setting managed lane pricing and for capturing traveler's behaviors for use in dynamic traffic assignment (DTA)/simulation modeling. These models are normally used to simulate traveler's route selection behaviors at 15 to 30 minute intervals and are being extended to include reliability in their generalized cost functions. In addition, the analyses of the Highway Capacity Manual (HCM) (HCM, 2010) are also conducted at the 15 minute analysis level and 
although the procedures of the current version of the manual do not consider reliability metrics, discussion has already started for the potential inclusion in future versions. This above discussion supports the argument that the reliability metrics need to be assessed at fine grained levels of aggregation for an increasing number of applications.

\subsection{User Behavior and the Value of Time}

While the value of time (VOT) is a very important notion in transportation planning and infrastructure management, it is a latent theoretical construct that cannot be easily quantified or measured. Different socioeconomic characteristics, trip purpose, and other attributes result in very heterogeneous traveler populations and therefore potentially in very different VOT values across individuals. For example, affluent travelers may be willing to pay a high toll to save trip time, while low income travelers may not have this option. One approach to quantify VOT is to develop discrete choice models based on data collected by surveys and then use the estimated coefficients for the cost and duration of travel to compute a VOT measure.

The interpretation of value of time depends on how the time allocation problem is modeled. Johnson (1966) for example, views travel time only as a cost thus keeping it out of the utility function. DeSerpa (1971), allows time to be included in the utility function and thus has a commodity value. Different ways of modeling the consumers' utility maximization problem has a profound effect on the way the value of time is measured. When time is only included in the constraint function, it is treated only as cost and acts as a budgetary constraint to consumption. In this case, time does not have a value per se and can't directly affect the utility. When time is included in the utility function, time gains 
commodity value and can directly influence consumer's utility. As a result, aside from its time constraint aspect, travel time can also cause a direct utility or disutility to travelers. In Johnson's formulation of the problem (Johnson, 1966), for example, commuting time does not yield any disutility, while in DeSerpa formulation (1971) it can.

In another study, Reichman (1976) concludes that time savings are just as important to the travelers as other attributes of the trip, such as comfort, scenery, physical, and mental efforts. Accordingly, it is possible that traveling on tolled roads provides less disutility for the motorists, in the form of a more pleasurable, less stressful ride due to improved driving conditions. Thus, people sometimes choose slower but more pleasant travel options, reflecting their lower total time cost, or they can be willing to pay extra for more comfortable seats, air conditioning, reduced transfers, etc. (Litman, 2007). As a result, it often becomes difficult to separate the pure value of travel time from other trip attributes affecting travelers' willingness to pay. Hensher (1976) argues that most empirical studies of travel mode choices fail to separate the pure value of time, which is mode-abstract, from the comfort and convenience features of the trip that affect the composite value of travel time.

Litman (2007) argues that while making their choices, travelers react to the perceived travel time, which can be different from actual travel time. Travelers often tend to overstate congestion-related delays and understate the benefits from saving travel time. This can lead to the differences in travel time estimates obtained from empirical studies based on stated preferences and revealed preferences. Since trade-off decisions are made at the margin, researchers argue that the value of time depends mostly on the marginal wage rate, while the average wage can provide only an approximation (Gronau, 1976). 
Finally, some researchers also point to the phenomenon that people often place value exclusively on the ability to control their time, irrespective of its utility or opportunity cost (Reichman, 1976). Thus, the value consumers place on saving time has more dimensions than simply opportunity cost of time measured by the money wage rate.

In the past decade, several VOT studies have been also conducted in Europe, including the Netherlands (Gunn et al., 1996), Norway (Ramjerdi et al. 1997), Sweden (Alger et al. 1996), the United Kingdom (Gunn et al., 1996), and Switzerland (Axhausen et al., 2004). Wardman (Wardman et al., 1998) presents a meta-analysis of VOT derived from 105 travel demand studies using revealed-preference and/or stated-preference methods. In conclusion, most of the studies aiming at the estimation of VOT for freight and passenger travel use discrete choice models. Due to practical reasons, most studies use logit models, while some recent studies (Bierlaire et al., 2005) use more advanced models such as mixed logit.

In conclusion, the literature review of the theoretical models found that there is no unique definition of the value of time. Through the review of the literature, it is shown that the value of time hinges on the assumption of a minimum-time constraint that requires individuals to travel more than they would choose to travel without the constraints. The literature is in continuous evolution with recent efforts aimed at presenting unified frameworks of analysis that explicitly account for this minimum-time constraint, and allow for the trade-off between discretionary and mandatory travel (Anas, 2007, Anas et. al., 1999). 


\subsubsection{Travel Time Perception and Learning in Transportation Systems}

The interdependency between the time-dependent behavior of traffic flows and users' choice behavior is central to the analysis and operation of intelligent transportation systems (ITS). Modeling and understanding the relationship between the transportation system performance perception, and the time-dependent behavior of traffic flows has received considerable attention from transportation researchers.

When making travel choices, drivers constantly combine various sources of information to perform perceptions and expectations of traffic conditions. For example when deciding the use of a priced facility, the user may combine his personal experiences, what he has heard about the facility, and in general any relevant information that can be used to support user decisions. The used information can be summarized in three categories:

(a) Historical information: information related to the state of the transportation system during previous time periods (previous time periods refer to experiences happened before the day when a decision is desired to be made).

(b) Current information: the most up-to-date information about current conditions.

(c) Predictive information: information concerning expected traffic conditions during subsequent time periods when travel can occur (subsequent time periods refer to the period immediately after a decision has been made)

In broad terms, in order to analyze the relationship between the transportation system performance and the time-dependent behavior of traffic flows, a dynamic driver behavior framework is required in order to capture the ways drivers process and use 
different sources of information in their decision making. The following section includes a deeper exploration of the components and relationships that need to be accounted for such dynamic driver behavior framework.

\subsubsection{Drivers Perception and Processing of Information}

There are several characteristics of the travelers that must be considered in the dynamics of information perception and learning. A framework to model individual perception and processing of information for decision making should account the fact that individuals have limited access to information (Hogarth, 1987; Newell et al.,1972), have limited capacity to process the information (Slovic, 1972), and attempts to find the best alternative within some time and effort constraints (Bettman, 1979; Heiner, 1983). In this sense, frameworks for driver behavior have been discussed by de Palma (de Palma et al., 1989) and Ben-Akiva (Ben-Akiva et al., 1991) and in both cases the main components of the driver decision process can be summarized as shown in Figure 2.2.

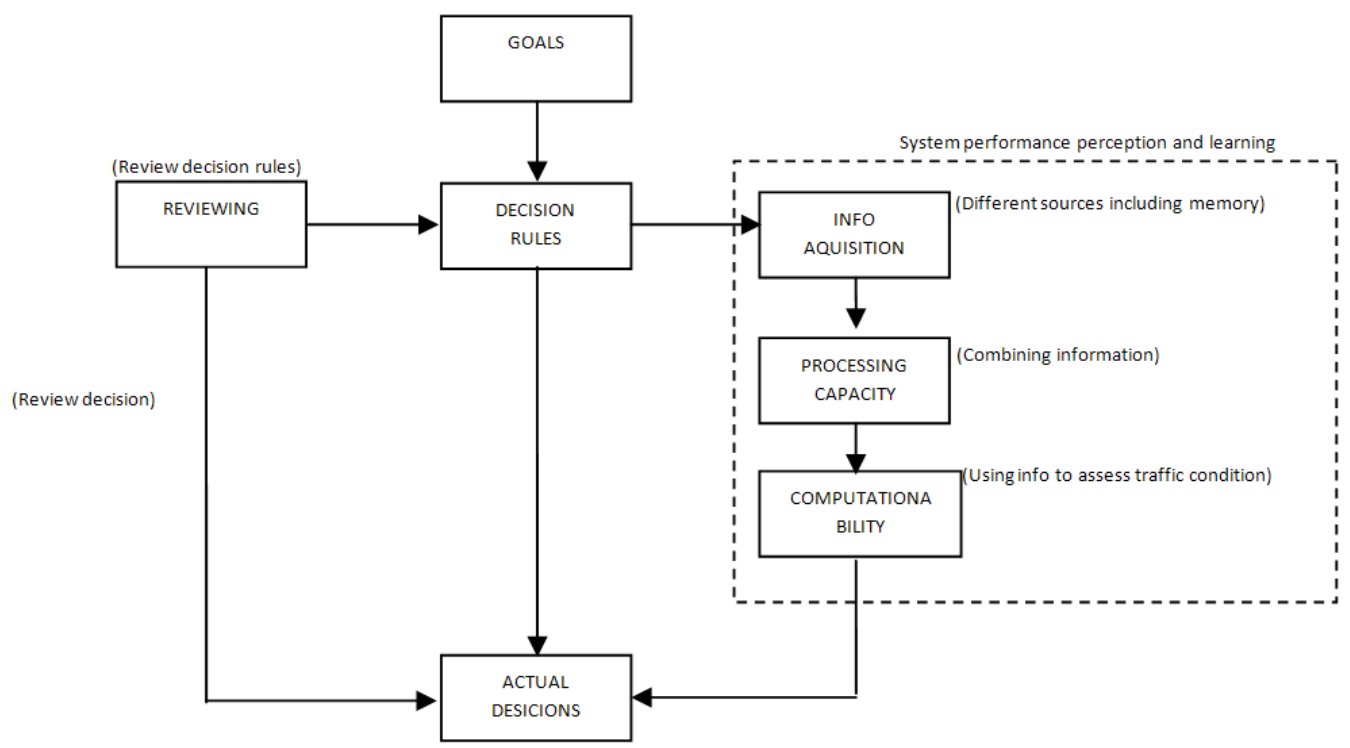

Figure 2.2: Driver Decision and Information Processing Components 
With the present study, the traveler's decisions are estimated based on ITS data archives. In this case, the decision rules (the parameters in a choice model) are estimated from the data by deriving a choice model that is a function of the historical time series of performance measures. Thus, the discussion in this section mainly focuses on "system performance perception and learning" in Figure 2.2, which reflects how the system performance is assessed by the transportation system users.

In Figure 2.2, the performance perception and learning block depicts the steps in how information is processed by drivers in order to assess his or her expectations about the transportation system performance. In particular, the information acquisition component refers to the fact that drivers can acquire historical information either by retrieving it from their own memory or by retrieving it from some database or collective memory. On the other hand, the processing capacity component captures the fact that different drivers have different abilities to combine and process a variety of information concerning road conditions. Finally the computational ability component addresses the fact that different drivers have also different abilities to use the available information, perform travel forecast, and develop heuristic decision procedures.

In the context of repetitive travel (such as commuting), the drivers dynamic behavior can be represented by a hierarchy of pre-trip and on route information perception and processing. In the pre-trip phase, an individual may integrate the previous day experience and information with his historic perceptions of travel time to form updated historic perceptions. Even before leaving the origin of a trip, a driver may acquire and process new day-specific traffic information based on media reports that may be used to update his expectations about the available choices. Once a trip begins, a 
driver acquires new information (with or without actively searching for it) about the quality of his choices. The driver may then actively decide and process new information and then make a new choice decision based on this information. This process is depicted in Figure 2.3 and the on-trip feedback is performed until the destination has been reached.

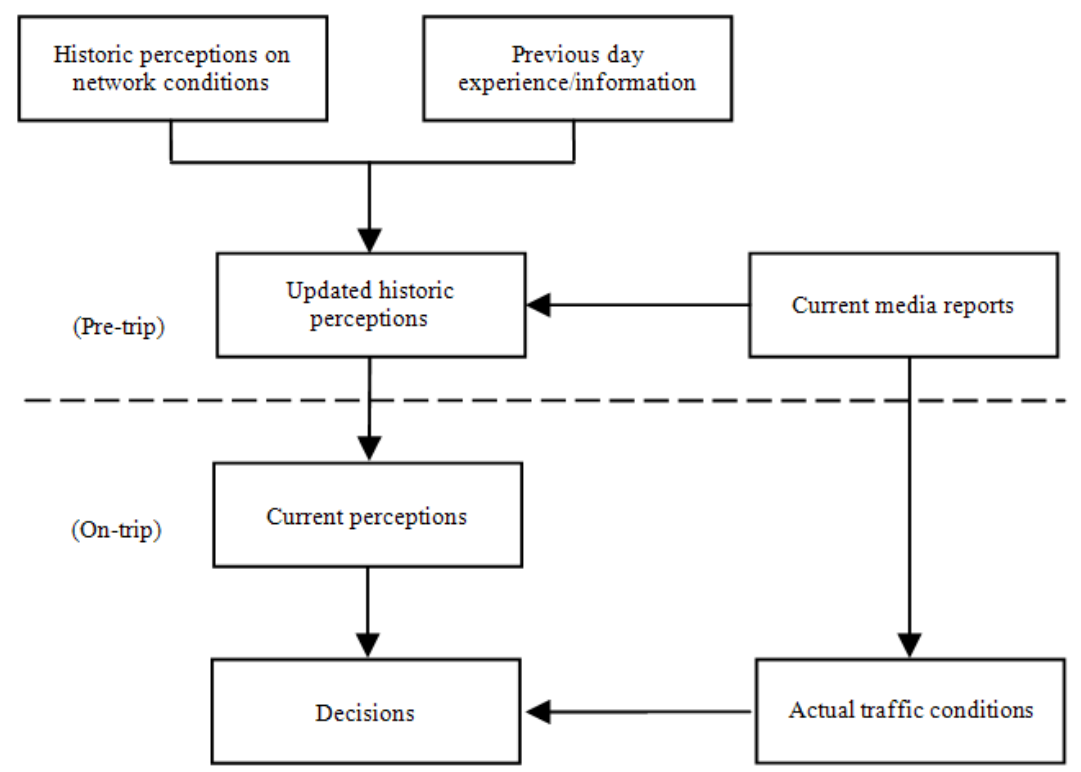

Figure 2.3: Drivers' Dynamic Acquisition and Processing of Information

\subsubsection{Travel Choices and Network Performance}

The question about revealing the interdependence between travel choices and network performance has been predominantly approached by assuming equilibrium under various assumptions about the user behavior. Although widely used in planning practice, equilibrium approaches have two main shortcomings. First, they rely heavily on the assumption that an equilibrium state exists and this state is unique, stable, and converges quickly, although no empirical evidence is available to support these assumptions under all conditions. Second, the effect of factors such as heterogeneity in users' behavior, learning and perception processes, are quite difficult to capture (Chen et al., 2003). 


\subsubsection{Learning Considering Equilibrium Approaches}

Extensions of the classical equilibrium framework to consider the day-to-day adjustment processes of traveler decisions were first explored by Beckman's seminal contribution to network modeling (Beckman et al. 1956). Day to day adjustment models of departure time and route decisions of commuters in response to experience and other sources of information were proposed by Mahmasani (Mahmassani et al., 1986; Mahmassani, 1990). Consideration of day to day adjustment resulted in the development of disequilibrium approaches to investigate the transportation system's dynamic evolution and properties. Cascetta (Cascetta, 1989) proposed a Markov chain formulation for analyzing day-to-day route choice dynamics. Cascetta and Cantarella (1991) further extended this formulation to include within-day dynamics, and more recently (Cantarella et al. (1996) have derived conditions for the existence and uniqueness of an equilibrium state in various dynamic process models for probabilistic assignment. A trial and error adjustment process model, based on optimal control theory, has also been proposed, but no user behavioral models were embedded in the equations describing the day-to-day dynamics (Friesz et al., 1994).

\subsubsection{Learning by means of simulated system}

Another approach to investigate the relationship between travel choices and network performance, which is recently gaining attention, consists of studies that either simulate the network conditions in response to decisions from real "actual" commuters (Mahmassani et al., 1986; Mahmassani H.S. 1990; Helbing et al., 2002), or simulate both the network and individual users decisions under various performance learning mechanisms and sources of information (Mahmassani et al., 1986; Peeta et al., 2001; Lu 
et al., 2010. Avineri et al., 2006; Iida et al., 1992). These studies attempt to circumvent the difficulty faced by equilibrium and disequilibrium approaches in capturing user behavior at the desired level of richness, simultaneously with measurements of prevailing conditions. Additionally, these approaches provide the ability to investigate the dynamic system evolution, in particular convergence and stability, and the mechanisms underlying the day-to-day choice behavior of users. Although one shortcoming of the experimental approach is the difference between user behavior in a simulated environment and in a real network, for the daily commuting decision environment these experiments are quite amenable since all participants are working commuters themselves and the route choice decision is typically made daily.

\subsubsection{Perception and Integration of Travel Choices and System Performance}

Aside from the relationship between travel choices and system properties, the perception and integration of travel information have been studied, though to a much lesser extent than traveler choice processes. Since past experiences are likely to influence user perception of network performance, modeling the mechanisms by which individuals integrate past experiences and information from other sources is important. More generally, information processing, integration and learning, and their role in decision-making and judgment have been of interest to marketing professionals (Bagozzi et al., 1983) and behavioral decision theorists like Einhorn and Hogarth or Ariely and Zauberman who have extensively addressed the integration of experience and information, and its role in decision-making (Hogarth, 1987; Einhorn et al., 1981; Ariely et al., 2000). Recent research on how people summarize and evaluate extended 
experiences indicates that they do not simply combine the intensity of their actual experiences (Ariely et al., 2000). Instead, the studies point out that individuals extract only a few defining features (Gestalt characteristics) of their experiences, which they combine into overall summary evaluations of the sequences (Kahneman et al., 1993; Carmon et al., 1996). Such features may include particularly salient characteristics such as the most intense state (peak) and the final state (end) of the experience.

In transportation, this choice behavior issue has received limited attention. Particularly, in the context of route choice in a network assignment the problem has been addressed by Horowitz (1984). In his study, the author suggests a process, where past experienced costs are integrated according to a weighted average, and finds that even under this reasonable rule, the system may not converge to an equilibrium state. Mahmassani and Chang (1986) examine a myopic adjustment and experience-based model of perceived travel time for departure time choice. Under myopic adjustment rule, the perceived travel time is a function of the latest day's outcome exclusively. The experience-based model is similar to the average rule suggested by Horowitz (1984). They find that convergence occurs only when all users are satisfied with their departure times within a tolerable limit, and interestingly that using the experience-based rules does not always lead to convergence as expected. Another contribution by Ben-Akiva et al.(1991) proposes a model where the updated perceived travel time is a weighted average of the historically perceived travel time and the time provided by advanced traveler information systems (ATIS), where the weights indicates the relative importance of historical and the real travel time information provided. Although all the models previously described address the individual travel time perception and updating 
mechanisms, these models do not account for the uncertainty associated or variance associated with travel times. The variance and uncertainty associated with travel time estimates is important, since they may significantly affect an individual's sense of a path's reliability. Additionally the above studies assume that perception does not vary with time.

\subsubsection{Accounting for Integration and Uncertainty in System Performance}

To account for both the integration of travel times and the associated uncertainty, Bayesian updating models have been proposed (Chen et al., 2003; Kaysi, 1991; Jha et al., 1998). A Bayesian statistical framework can account for updating both the estimate of the mean and variance in light of experience and information.

\subsubsection{A Model for Information Processing and Learning}

A model similar to the one proposed by Horowitz (Horowitz, 1984) is considered to assess the user learning processes and perception in their travel choice decisions. In this model, it is assumed that in each time period $t$, travel decisions are based on weighted averages of measured travel costs in previous day-to-day experiences. In other words:

$$
\hat{C}_{i t}=\sum_{k=1}^{t-1} w_{k} C_{i k}+\varepsilon_{i t}
$$

Where $\hat{C}_{i t}$ is the cost of travel on link $i$ as perceived by a randomly selected traveler at the time he/she decides which route to use in period $t ; C_{i t}$ is the measured or estimated cost of travel on link $i$ in time period $k ; \varepsilon_{i t}$ is a random variable whose probability distribution is independent of $t$; and $w_{k}$ is a non-negative weight. For each $t$, 
the weights $w_{k}(k=1, \ldots, t-1)$ satisfies:

$$
\sum_{k=1}^{t-1} w_{k}=1
$$

For each $i$ and $t$ define $\bar{C}_{i t}$ by:

$$
\bar{C}_{i t}=\sum_{k=1}^{t-1} w_{k} C_{i k}
$$

Then by referring to Equation 2.9 it can be seen that $\bar{C}_{i t}$ represents the composite effect of past measured link costs on current perceived link costs. The weights $w_{k}$ describe the relative influences of recent and distant past cost on current perceptions. For example, suppose that current perceptions are determined mainly by cost in the recent past and that costs in the more distant past are forgotten. Then it might be expected that for each $t$, $w_{1}<w_{2}<\ldots<w_{t-1}$. Conversely, suppose that travelers develop route choice habits early in their experience with a roadway network and that these habits are not easily altered by current events. Then it might be expected that for each $t, w_{1}>w_{2}>\ldots>w_{t-1}$. Since the relative influences of recent and distant past costs on the perception of travelers in real roadway networks are not necessarily known at present, models based on a variety of different weighting schemes will be discussed shortly. If some sort of real time information system is available, the model presented before can be further expanded in order to accommodate the influence of such real time information in the user perception of current travel cost in the link. Thus the perceived link cost can be written as:

$$
\bar{C}_{i t}=a_{i t} C_{i t}+\left(1-a_{i t}\right) \sum_{k=1}^{t-1} w_{k} C_{i k}
$$


where $a_{i t}$ is a sequence of constants that may be time and link specific satisfying $0 \leq a_{i t} \leq 1$. This model is flexible enough to accommodate a wide variety of weighting schemes of possible practical interest, including:

(a) Myopic approach in which actual link cost perception in time period $t$ depends on link costs in time period $t-1$ only in such case $w_{k}=0$, if $k \neq t-1, \quad w_{t-1}=1$, and $a_{i t}=0$ for all $t$

(b) Actual link cost perception in time period $t$ depends only on the arithmetic mean on link cost in previous time periods. In this case $w_{k}=1 /(t-1)$, and $a_{i t}=0$ for all $t$

(c) Actual link cost perception in time period $t$ depends only on exponentially decreasing weights from the present time into the past. The parameter $b$ represents how fast memory decays in time. In this case $a_{i k}=0$ for all $t$ and $w_{k}=b^{k} / \sum_{j=1}^{t-1} b^{j}$

(d) Actual link cost perception in time period $t$ depends partially on exponentially decreasing weights from the present time into the past and partially on real time information broadcasted by ATIS systems, in this case $w_{k}=b^{k} / \sum_{j=1}^{t-1} b^{j}$, and $a_{i t} \neq 0$ for all $t$. 


\subsection{Intelligent Transportation System (ITS) Data}

ITS agencies have used devices such as traffic detectors, closed circuit television cameras (CCTV), electronic toll readers, and license plate readers to collect traffic parameter measurements for operational purposes. In recent years, these agencies have started archiving the data collected by these devices (FHWA, 2004). Because ITS detectors and associated communication systems are already in place to collect data for operational purposes, the extra cost to archive and manage the data is relatively low. As ITS data archives become more widely available, the utilization of such archives for the development and calibration of simulation applications is an attractive option. This utilization provides a significantly lower cost and a more efficient data collection method compared to traditional methods and increases safety by reducing the need for personnel to go out to the field for data collection purposes. The additional details provided by the ITS data, both in time and space resolutions, allow better representations of real-world environments in simulation applications. For example, the use of archived ITS data allow the simulation of seasonal variations in traffic, special events, accidents, work zones, weather events, other types of incidents, and incident management strategies.

The Statewide Transportation Engineering Warehouse for Archived Regional Data (STEWARD) has been developed as a proof of concept prototype for the collection and use of ITS data in Florida (Courage, 2008). The development of this prototype has demonstrated that data from traffic management centers around Florida can be centrally archived in a practical manner and that a variety of useful reports and other products can be produced. The STEWARD system archives data in a database that supports the generation of summary reports and queries. The current effort has concentrated on 
archiving point traffic detector data and travel time estimates. STEWARD contains summaries of traffic volumes, speeds, and occupancies collected from point traffic detectors. These detectors are located every 0.3-0.5 miles along the equipped corridors. The stored data can be aggregated by 5, 15 and 60 minute periods, as requested by the user. Reports are generated at the detector, detection station, and system levels. The travel times in the archive are those estimated by the traffic management central software in Florida (the SunGuide software) based on data collected from the point traffic detectors. STEWARD includes a quality assessment procedure to identify bad or suspicious data.

Alvarez (Alvarez et al., 2010) discussed a series of data manipulation procedures for the utilization of ITS data contained in the STEWARD system to support simulation modeling. These procedures allow the extraction of collected volume data from ITS data archives, automatic identification of temporal patterns in the data, automatic segmentation of daily demands into dynamically captured sub-periods to best fit the variations in the demands, resolving possible spatial inconsistencies in the data, and estimating missing volumes. The archived data is shown to be useful for the estimation of a variety of input parameters required to adjust simulation models to particular environmental or traffic conditions.

\subsection{Summary of the State of the Art}

Road pricing is an important topic that has been extensively investigated. Various approaches have been applied to improve road pricing modeling. Generally speaking, the current state of the practice of road pricing analysis and design is not 
adequate for satisfactory operational analysis or real time traffic management. Undoubtedly, the most promising directions for principal improvements in road pricing modeling are associated with advanced network modeling tools such as dynamic traffic assignment combined with mesoscopic simulation and micro-simulation modeling tools, and in some cases with advanced activity-based demand models. These advanced tools have a much higher flexibility in assessing various impacts of road pricing on travel behavior compared to conventional tools like the four step modeling tools and the associated static assignment models. More specifically, there is potential for building on recent research results related to the heterogeneity of road users with respect to their willingness to pay, for the impacts of the reliability of travel time associated with toll roads, the time-of-day choice, and the proper accounting for pricing in various steps of the travel choice modeling process.

Finally, it should be highlighted that data collected by ITS devices are increasingly available. However, the use of ITS data to support transportation modeling in general and congestion pricing in particular is still at its earlier stages. 


\section{CHAPTER 3}

\section{PARAMETER ESTIMATION OF DYNAMIC SYSTEMS}

One of the main requirements of this study is to develop a model to estimate time-varying travel behavior as a function of time based on ITS data. However, most parameter estimation techniques process the data in a batch mode making it difficult to produce time varying parameters that incorporate information as received in real time from measurement devices. Filtering techniques have been developed to specifically address the estimation of state parameters of a system based on continuous measurement data. In this section, such filtering techniques are discussed and further extension of the well-known discrete Kalman filter is developed in order to process traffic performance measures and observed proportion of the priced system to produce time varying estimates of the aggregated traveler behavior.

\subsection{Parameter Estimation Basics}

Dynamic systems are common in the real-world; such as in the case of many biological, electrical, civil, chemical, aerospace, road traffic, and a variety of other systems. Parameter estimation is the process of using observations from a dynamic system to develop mathematical models that adequately represent the system characteristics. The assumed model consists of a finite set of parameters, the values of which are estimated using estimation techniques.

Mathematical modeling for parameter estimation is one way to achieve a deeper understanding of the system characteristics. The problem of parameter estimation belongs to a class of "inverse problems" in which the knowledge of the dynamical system is 
derived from the input-output data of the system (Raol et al., 2004). In general, the problem of parameter estimation is based on minimization of some criterion, and this criterion itself can serve as the means to establish the adequacy of the identified model. Figure 3.19 shows a simple approach to parameter estimation. In this approach, the parameters of the model are adjusted iteratively until such time the responses of the model match closely with the measured outputs of the system under investigation as specified by the minimization criterion. There are a number of different approaches used for parameter estimation: Least Squares, Maximum Likelihood, Bayesian estimation, discrete and continuous filters, Artificial Neural Networks (ANN), Genetic Algorithms (GA), and others. All of them differ in many ways; however, the key consideration to decide a particular approach has to do with the richness of available data and the desired characteristics of the parameters to be estimated.

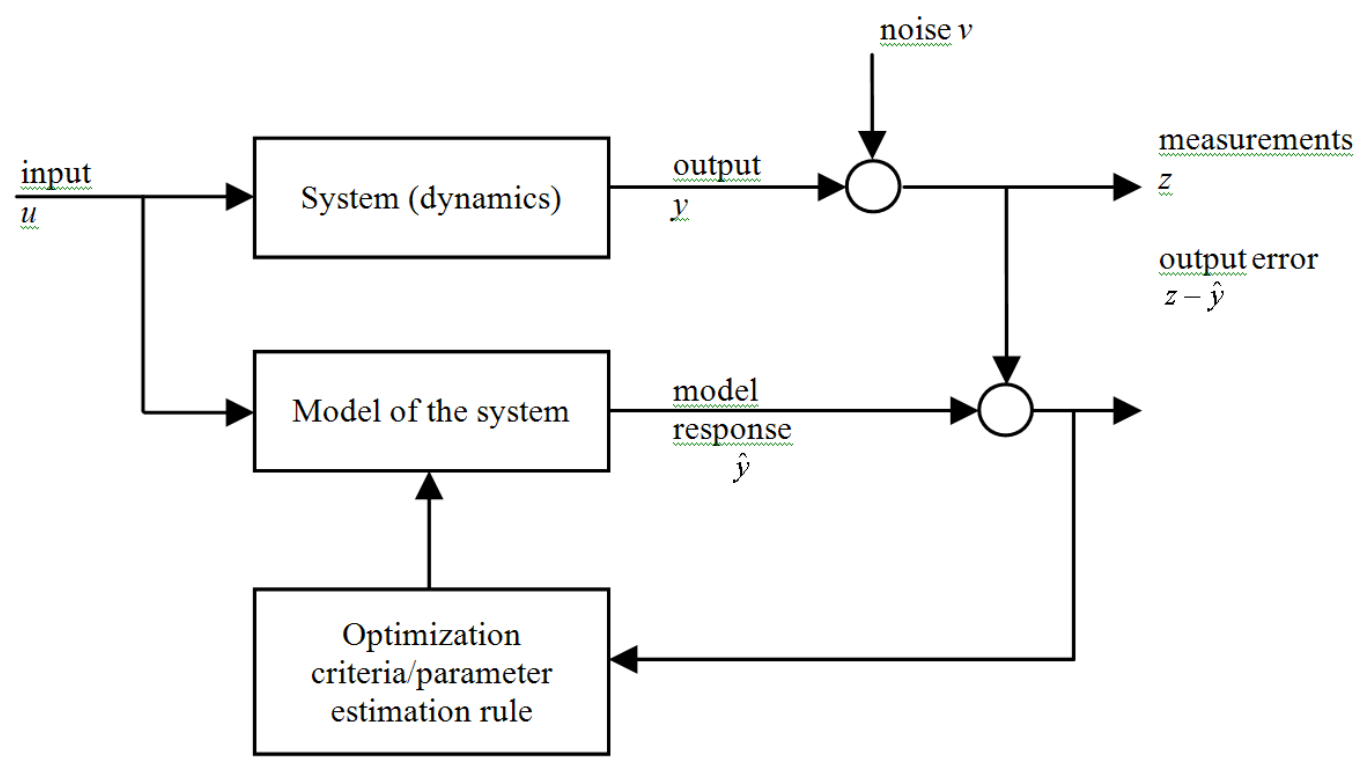

Figure 3.1: Simplified Block Diagram of the Estimation Procedure Approach 
This study requires estimating the time-varying parameters in the utility function that models the logic behind the revealed preferences between two travel choices, when the attributes of each available choice also vary with time. In addition to proper consideration of the desired time characteristics of the estimates, the estimation methodology should take into account the following problem characteristics:

- The model used to represent the user preferences may partially represent the actual logic on which to base decisions.

- The available data about revealed traveler preferences is noise corrupted by the characteristics of the sensor's technology, the characteristics of the methods used to smooth/inputted the data, and the randomness of the process.

- In the face of uncertain system descriptions, incomplete and noise-corrupted data and other disturbances beyond the analyst control, the methodology should produce reliable time-varying parameter estimates.

Parameter estimation methods considering the aforementioned characteristics of the problem are referred to in the literature as Optimal Estimation (OE). An optimal estimation algorithm processes measurements to deduce a minimum error (in accordance to some criterion) estimate of the state of a system by utilizing: knowledge of system and measurement dynamics, assumed statistics of the system noises and measurement errors, and initial condition information (Gelb, 1989). In particular when the optimal estimation coincides with the last measurement data point, the optimal estimation is referred as filtering. 
Probably the most common optimal filtering technique is that developed by Kalman (1960) for estimating the state of a linear system from noise measurements. The Kalman filter (KF) is an optimal state estimator applied to a dynamic system that involves random noise and includes a limited amount of noisy real-time measurements. Although originally derived for linear systems, KF can also be extended for a number of interesting problems. The most straightforward extension is the Extended Kalman Filter (EKF) for non-linear systems (Kalman, 1960; Gelb, 1989). A special case of the EKF with very convenient computational properties is the Limiting Extended Kalman Filter (LimEKF) (Chui et al., 1999). The Unscented Kalman Filter (UKF) (Julier et al., 1995) is an alternative filter where the main difference with the EKF is in the representation of the random variables for propagation through the system dynamics.

\subsection{The Discrete Kalman Filter}

This section describes the filter in its original formulation (Kalman, 1960) where the measurements occur and the state is estimated at discrete points in time.

\subsubsection{The Process to be Estimated}

The Kalman filter addresses the general problem of trying to estimate the state $x \in R^{n}$ of a discrete-time controlled process that is governed by the linear stochastic difference equation:

$$
x_{k}=A x_{k-1}+w_{k-1}
$$

with a measurement $z_{k} \in R^{m}$ that is

$$
z_{k}=H x_{k}+v_{k}
$$


The random variables $w_{k-1}$ and $v_{k}$ represent the process and measurement noise (respectively). They are assumed to be independent (of each other), white, and with normal probability distributions:

$$
\begin{aligned}
& p(w) \sim N(0, Q) \\
& p(v) \sim N(0, R)
\end{aligned}
$$

In practice, the process noise covariance $Q$ and measurement noise covariance $R$ matrices might change with each time step or measurement, however here we assume they are constant. Later in this section, this assumption is released and the estimation is performed assuming a time varying covariance in Equations 3.3 and 3.4

The $n \times n$ matrix $A$ in the difference Equation 3.1 relates the state at the previous time step $k-1$ to the state at the current step $k$ in the absence of either a driving function or process noise. Note that in practice, $A$ might change with each time step, but here we assume it as constant. The matrix $H$ in the measurement Equation 3.2 relates the state to the measurement $z_{k}$. In practice, $H$ changes with each time step or measurement, reflecting the time varying character of the explanatory variables (travel time, reliability, toll) considered.

\subsubsection{The Computational Origin of the Filter}

We define $x_{k}^{-} \in R^{n}$ (note the "super minus") to be our a priori state estimate at step $k$ given knowledge of the process prior to step $k$, and $\hat{x}_{k} \in R^{n}$ to be our a posteriori state estimate at step $k$, given measurement $z_{k}$. We can then define a priori and a posteriori estimate errors as: 


$$
\begin{gathered}
e_{k}^{-} \equiv x_{k}-\hat{x}_{k}^{-} \\
e_{k} \equiv x_{k}-\hat{x}_{k}
\end{gathered}
$$

the a priori estimate error covariance is then:

$$
P_{k}^{-} \equiv E\left[e_{k}^{-} e_{k}^{-T}\right]
$$

and the a posteriori estimate error covariance is:

$$
P_{k} \equiv E\left[e_{k} e_{k}^{T}\right]
$$

In deriving the equations for the Kalman filter, we begin with the goal of finding an equation that computes a posteriori state estimate $\hat{x}_{k}$ as a linear combination of an $a$ priori estimate $\hat{x}_{k}^{-}$and a weighted difference between an actual measurement $z_{k}$ and a measurement prediction $H x_{k}^{-}$as shown below in Equation 3.9.

$$
\hat{x}_{k}=\hat{x}_{k}^{-}+K\left(z_{k}-H \hat{x}_{k}^{-}\right)
$$

The difference in Equation 3.9 is called the measurement innovation, or the residual. The residual reflects the discrepancy between the predicted measurement $H \hat{x}_{k}^{-}$ and the actual measurement $z_{k}$. A residual of zero means that the two are in complete agreement.

The $n \times m$ matrix $K$ in Equation 3.9 is chosen to be the gain or blending factor that minimizes the a posteriori error covariance Equation 3.8. This minimization can be accomplished by first substituting Equation 3.9 into the above definition for $e_{k}$, substituting that into Equation 3.8, performing the indicated expectations, taking the derivative of the trace of the result with respect to $K$, setting that result equal to zero, and then solving for $K$. One form of the resulting $K$ that minimizes Equation 3.8 is given by: 


$$
K_{k}=P_{k}^{-} H^{T}\left(H P_{k}^{-} H^{T}+R\right)^{-1}
$$

One way to think about the weight $K$ is that as the measurement error covariance $R$ approaches zero, the actual measurement $z_{k}$ is "trusted" more and more, while the predicted measurement $H \hat{x}_{k}^{-}$is trusted less and less. On the other hand, as the $a$ priori estimate error covariance $P_{k}^{-}$approaches zero, the actual measurement $z_{k}$ is trusted less and less, while the predicted measurement $H \hat{x}_{k}^{-}$is trusted more and more.

\subsubsection{The Discrete Kalman Filter Algorithm}

The Kalman filter estimates a process by using a form of feedback control: the filter estimates the process state at some time and then obtains feedback in the form of noisy measurements. As such, the equations for the Kalman filter fall into two groups: time update equations and measurements update equations. The time update equations are used for projecting forward (in time) the current state and error covariance estimates to obtain the a priori estimates for the next time step. The measurements update equations are used for the feedback; this is, for incorporating a new measurement into the a priori estimate to obtain an improved a posteriori estimate. The specific equations for the time and measurement updates are presented below in Table 3.1 


\section{Table 3.1: Discrete Kalman Filter Equations}

\begin{tabular}{|l|}
\hline \multicolumn{2}{|c|}{ Time update equations } \\
\hline$x_{k}=A x_{k-1}+w_{k-1}$ \\
\hline$P_{k}^{-}=A P_{k-1} A^{T}+Q$ \\
\hline \multicolumn{2}{|c|}{ Measurement update equations } \\
\hline$K_{k}=P_{k}^{-} H^{T}\left(H P_{k}^{-} H^{T}+R\right)^{-1}$ \\
\hline$\hat{x}_{k}=\hat{x}_{k}^{-}+K_{k}\left(z_{k}-H \hat{x}_{k}^{-}\right)$ \\
\hline$P_{k}=\left(I-K_{k} H\right) P_{k}^{-}$ \\
\hline
\end{tabular}

The first task during the measurement update is to compute the Kalman gain, $K_{k}$

(Equation 3.13). The next step is to actually measure the process to obtain $z_{k}$, and then

to generate an a posteriori state estimate by incorporating the measurement as in Equation 3.14. The final step is to obtain an a posteriori error covariance estimate via Equation 3.15.

After each time and measurement update pair, the process is repeated with the previous a posteriori estimates used to project or predict the new a priori estimates. This recursive nature is one of the very appealing features of the Kalman filter.

\subsection{Adaptive Kalman Filter}

It is a well known limitation in applying Kalman filter techniques that errors of the process and measurement noise statistics are assumed to be known. The use of wrong $a$ priori statistics can lead to large estimation errors or even to a divergence of errors. The purpose of an adaptive filter is to reduce or bound the errors by modifying or adapting the Kalman filter to real data. In the case of this study, there is no information a priori about the time varying characteristics of the process and measurement noise statistics. In general, we consider that the measurement error can change due to several reasons: 
- Detector technology, power failures and, maintenance issues

- Weather conditions

- Traffic variability (low/high congestion).

As an illustration of the previous discussion, Figure 3.2 shows the measurement variance as a function of the time of day for a typical instance of the dataset. The figure presents the variance estimates based on 30 and 60 minutes worth of data (5 minutes aggregation level). Based on the figure it is possible to find consecutive periods of time with significant differences in variability. Also the magnitude of the variability differences depend on the time window size time considered for collecting the samples of the measurement data.

On the other hand, for the process noise statistics, the covariance may change due to:

- Changes in user behavior because of time of the day and day of the week (trip purposes and possibly changes in recurrent congestion)

- Changing in user behavior due to traffic incidents (accidents, work zones, special events)

- Changing in user behavior due to weather events.

Unfortunately, there is no a priori information relative to the time-varying characteristics of the process noise variability illustrating the need to count with an algorithm able to cope with such variability. However, realizing the occurrence of the behavioral changes and their impacts described above lead to the decision to utilize an estimation procedure that automatically adapts to real data in order to minimize the estimation errors. What follows is a procedure that allows the sequential estimation of the process and measurement noise statistics in order to accomplish such goal. 

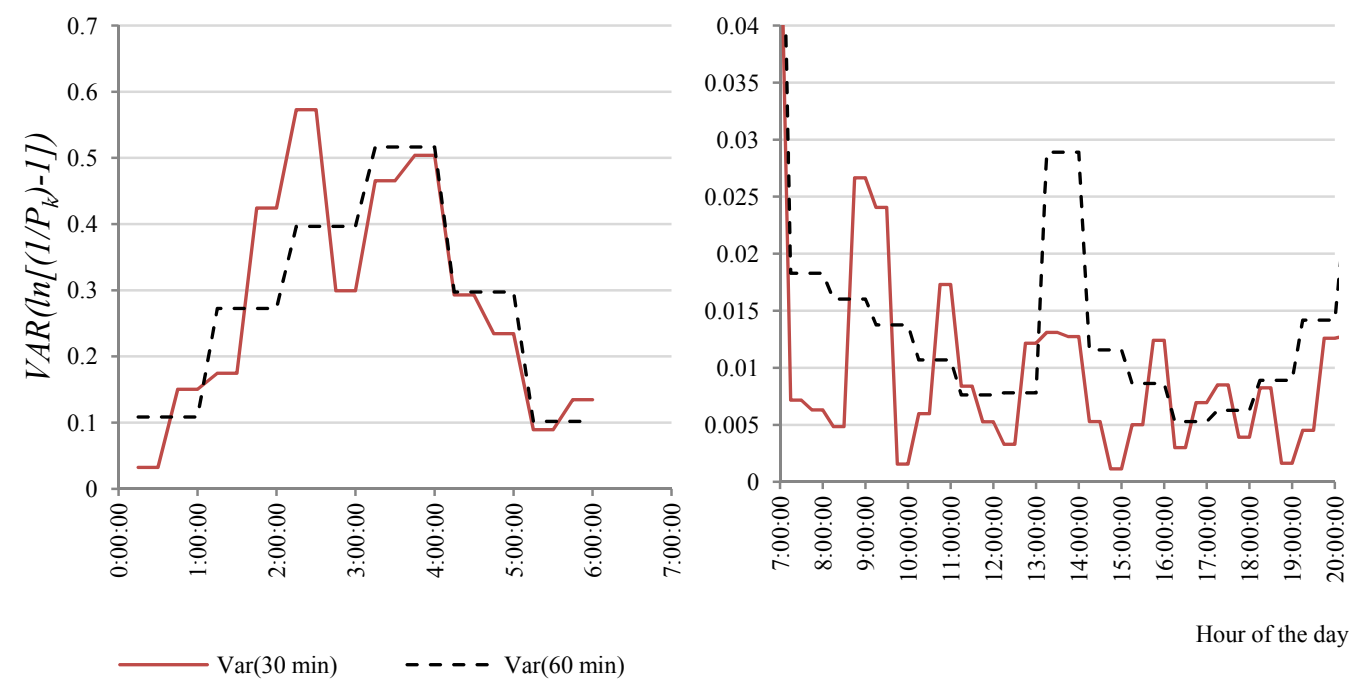

Figure 3.2: Measurement Variance Variation along the Day

\subsubsection{Sequential Estimation with Unknown Noise Statistics}

For a linear conventional Kalman filter, its state covariance matrix is totally determined by given initial conditions and $P$ and $Q$ values without direct link to the actual measurements. In contrast, the covariance matrix of an adaptive Kalman filter may change during the filtering process because it is partially derived from actual measurements which correspond to different settings.

A number of approaches can be taken to adaptive filtering. Since the basic source of uncertainty is due to unknown a priori statistics of noise, one can try to estimate them on-line from the observed data. Different approaches to adaptive filtering have being described to belonging to one of four methodological categories (Mehra, 1972): Bayesian, maximum likelihood, correlation, or covariance matching. Unfortunately, an optimal estimator for the state noise and observation noise does not exist, but many suboptimal schemes have been developed which estimate one or more of the parameters 
simultaneously with the state (Mehra, 1972; Jazwinski, 2007; Raol et al., 2004). Most of these developments, however, are too restrictive or computationally demanding, especially for nonlinear applications (Myers et al., 1976). For example, some approaches require that the unknown statistics be restricted to a set of constant parameters (Hilborn et al., 1969), that the noise covariance matrix be diagonal (Mehra, 1972), or that the state transition and geometry matrices be time invariant (Alspach, 1974). Other techniques (Jazwinski, 2007) require iteration or smoothing which can be computationally burdensome. In this work, a more intuitive approach is taken which is similar to those in the category of covariance matching. The particular method given here largely follows Myers \& Tapley (1976).

The system considered here is the linear discrete, stochastic sequence described by the following equations:

$$
\begin{aligned}
& x_{k}=A_{k-1, k} x_{k-1}+w_{k-1} \\
& z_{k}=H_{k} x_{k}+v_{k}
\end{aligned}
$$

where $x_{k}$ is the n-dimensional state vector, $A_{k-1, k}$ is the state transition matrix from time $t_{k-1}$ to $t_{k}, z_{k}$ is the $m$-dimensional observation vector, and $H_{k}$ is the observation mapping matrix. The stochastic disturbance vectors $w_{k-1}$ and $v_{k}$ are treated as independent, non-stationary, Gaussian, white noise sequences with the following properties:

$$
\begin{aligned}
& E\left[w_{i}\right]=q_{i} \\
& E\left[\left(w_{i}-q_{i}\right)\left(w_{j}-q_{j}\right)^{T}\right]=Q_{i} \delta_{i j} \\
& E\left[v_{i}\right]=r_{i},
\end{aligned}
$$




$$
E\left[\left(v_{i}-r_{i}\right)\left(v_{j}-r_{j}\right)^{T}\right]=R_{i} \delta_{i j}
$$

where $q_{i}$ and $r_{i}$ are the true means and $Q_{i}$ and $R_{i}$ are true moments about the mean of the state and observation noises respectively.

\subsubsection{Empirical Estimation of Measurement Noise Statistics}

For the measurement noise statistics, consider the linear observation state relationship at a given observation time $t_{k}$ (Equation 3.17). The true state $x_{k}$ is unknown, so $v_{k}$ cannot be determined; but an intuitive approximation for $v_{k}$ is given by the quantity:

$$
r_{k}=z_{k}-H_{k} x_{k}^{-}
$$

where $r_{k}$ is defined as the observation noise sample and $x_{k}^{-}$is the a priori state knowledge.

If the noise samples $r_{k}$ are considered to be representative of $v_{k} \sim N(r, R)$ they may be considered independent and identically distributed, and a simple parameter estimation problem can be constructed. Define a random variable $\Re$ on the sample space $\Omega_{\Re}$ from which the data $r_{j}, j=1 \rightarrow n$ are obtained. Based on these empirical measurements, the unknown distribution of $\Re$, characterized by a mean $r$ and covariance $C_{r}$ is to be estimated.

An unbiased estimator for $r$ is taken as the sample mean,

$$
\widehat{r}=\frac{1}{N} \sum_{j=1}^{N} r_{j}
$$

An unbiased estimator for $R$ is obtained by first constructing an estimator for $C_{r}$ the covariance of $\mathfrak{R}$ as follows, 


$$
\widehat{C}_{r}=\frac{1}{N-1} \sum_{j=1}^{N}\left(r_{j}-\widehat{r}\right)\left(r_{j}-\widehat{r}\right)^{T}
$$

It can be shown that expected value of this quantity is

$$
E\left[\widehat{C}_{r}\right]=\frac{1}{N} \sum_{j=1}^{N} H_{j} P H_{j}^{T}+R
$$

An unbiased estimate of $R$, after substitution of Equation 3.29 is given by

$$
\widehat{R}=\frac{1}{N-1} \sum_{j=1}^{N}\left\{\left(r_{j}-\widehat{r}\right)\left(r_{j}-\widehat{r}\right)^{T}-\left(\frac{N-1}{N}\right) H_{j} P_{j} H_{j}^{T}\right\}
$$

\subsubsection{Empirical Estimation of State Noise Statistics}

For the state noise statistics, consider the linear dynamical state relation Eq. (3.17) at given time $t_{k}$ and $w_{k-1} \sim N(q, Q)$. The true states $x_{k}$ and $x_{k-1}$ are unknown, so $w_{k-1}$ cannot be determined, but an intuitive approximation for $w_{k-1}$ is:

$$
q_{k}=\widehat{x}_{k}-A_{k-1} \widehat{x}_{k-1}
$$

where $q_{k}$ is defined as the state noise sample at time $t_{k}$. By hypothesis, the $w_{k-1}$ for $k=1 \rightarrow n$ are independent, and the parameters $q$ and $Q$ are constant. If the $q_{k}$ are assumed to be representative of the $w_{k-1}$, they may be considered independent and identically distributed. Again defining a parameter estimation problem, let $\mathfrak{I}$ be a random variable on the sample space $\Omega_{\Im}$ from which the data $q_{k}$ is being obtained, $j=1 \rightarrow n$. Based on these measurements, the unknown distribution of $\mathfrak{I}$, characterized by $q$ and $Q$ is to be estimated. 
An unbiased estimator for $q$ is the sample mean

$$
\hat{q}=\frac{1}{N} \sum_{j=1}^{N} q_{j}
$$

An unbiased estimator for $Q$ is obtained by first constructing the estimator for $C_{q}$ the covariance of $\mathfrak{I}$

$$
\widehat{C}_{q}=\frac{1}{N-1} \sum_{j=1}^{N}\left(q_{j}-\hat{q}\right)\left(q_{j}-\hat{q}\right)^{T}
$$

Following the same steps than the measurement noise statistics, the unbiased estimator for $Q$ is given by:

$$
\widehat{Q}=\frac{1}{N-1} \sum_{j=1}^{N}\left\{\left(q_{j}-\hat{q}\right)\left(q_{j}-\widehat{q}\right)^{T}-\left(\frac{N-1}{N}\right)\left[A_{j} P_{j-1} A_{j}^{T}-P_{j}\right]\right\}
$$

\subsubsection{The Kalman Adaptive Memory Limited Filter (AMLF) Algorithm}

This section describes the modifications to the KF algorithm and the unknown statistics sequential estimation techniques required for application to real problems.

Empirical estimators of the unknown statistics are derived in a batch form, assuming that during a given period of time (LAP) such statistics remain constant. If we call that period of time LAP, the KF algorithm needs to be modified on order to allow the computation of such statistics during LAP period of time as new measurement data is processed. The AMLF algorithm is shown in Table 3.2 
Table 3.2: ALMF Algorithm

\begin{tabular}{|c|c|}
\hline $\begin{array}{c}\text { A priori } \\
\text { data }\end{array}$ & $\hat{x}_{0}, \hat{P}_{0}, \hat{q}_{0}, \hat{Q}_{0}, \hat{r}_{0}, \hat{R}_{0} ; k=1$ \\
\hline $\begin{array}{c}\text { State } \\
\text { Propagation }\end{array}$ & $x_{k}^{-}=\hat{x}_{k-1}+\hat{q}_{k-1}$ \\
$P_{k}^{-}=A \hat{P}_{k-1} A^{T}+\hat{Q}_{k-1}$ & $\hat{r}_{k}=z_{k}-H_{k} x_{k}^{-}$ \\
\hline $\begin{array}{c}\text { Compute } \\
\text { Observation } \\
\text { Noise }\end{array}$ & $\hat{R}_{k}=\frac{1}{L A P-1} \sum_{j=1}^{L A P}\left\{\left(r_{j}-\hat{r}\right)\left(r_{j}-\hat{r}\right)^{T}-\left(\frac{L A P-1}{L A P}\right) H_{j} P_{j} H_{j}^{T}\right\}$ \\
\hline $\begin{array}{c}\text { Compute } \\
\text { Kalman Gain }\end{array}$ & $K_{k}=P_{k}^{-} H_{k}{ }^{T}\left(H_{k} P_{k}^{-} H_{k}{ }^{T}+\hat{R}_{k}\right)^{-1}$ \\
\hline $\begin{array}{c}\text { State } \\
\text { Estimation }\end{array}$ & $\hat{x}_{k}=\hat{x}_{k}^{-}+K_{k}\left(z_{k}-H_{k} \hat{x}_{k}^{-}\right)$ \\
\hline $\begin{array}{c}\text { Compute } \\
\text { State Noise }\end{array}$ & $\hat{Q}=\frac{13.34)}{L A P-1} \sum_{j=1}^{L A P}\left\{\left(q_{j}-\hat{q}\right)\left(q_{j}-\hat{q}\right)^{T}-\left(\frac{L A P-1}{L A P}\right)\left[A_{j} \hat{P}_{j-1} A_{j}^{T}-\hat{P}_{j}\right]\right\}$ \\
\hline $\begin{array}{c}\text { Reset and } \\
\text { Repeat }\end{array}$
\end{tabular}




\section{CHAPTER 4}

\section{METHODOLOGY}

This research work aims at the development of methodology to assess the aggregated user behavior in a corridor managed using road pricing strategies. Generally speaking, the methodology makes use of historical ITS data to determine the relationship between the observed proportion of the HOT lanes users and the performance of the transportation system. Initially, the historical information on system performance in terms of travel time, travel time reliability, toll, and occupancy are processed in different manners to select the best method to capture their effects on the variation of the proportion of HOT users. These rules have the ability to incorporate historical and real time information into the final summary measure of interest. In addition, these rules allow the generation of travel time and travel time reliability series that describe the travelers' perception of the system performance as a function of time. Finally the performance of the ALMF proposed in Chapter 3 is tested regarding its accuracy and robustness. Figure 4.1 illustrate the methodology and its relationship to the estimation of the various traveler sensitivities in a priced environment. 

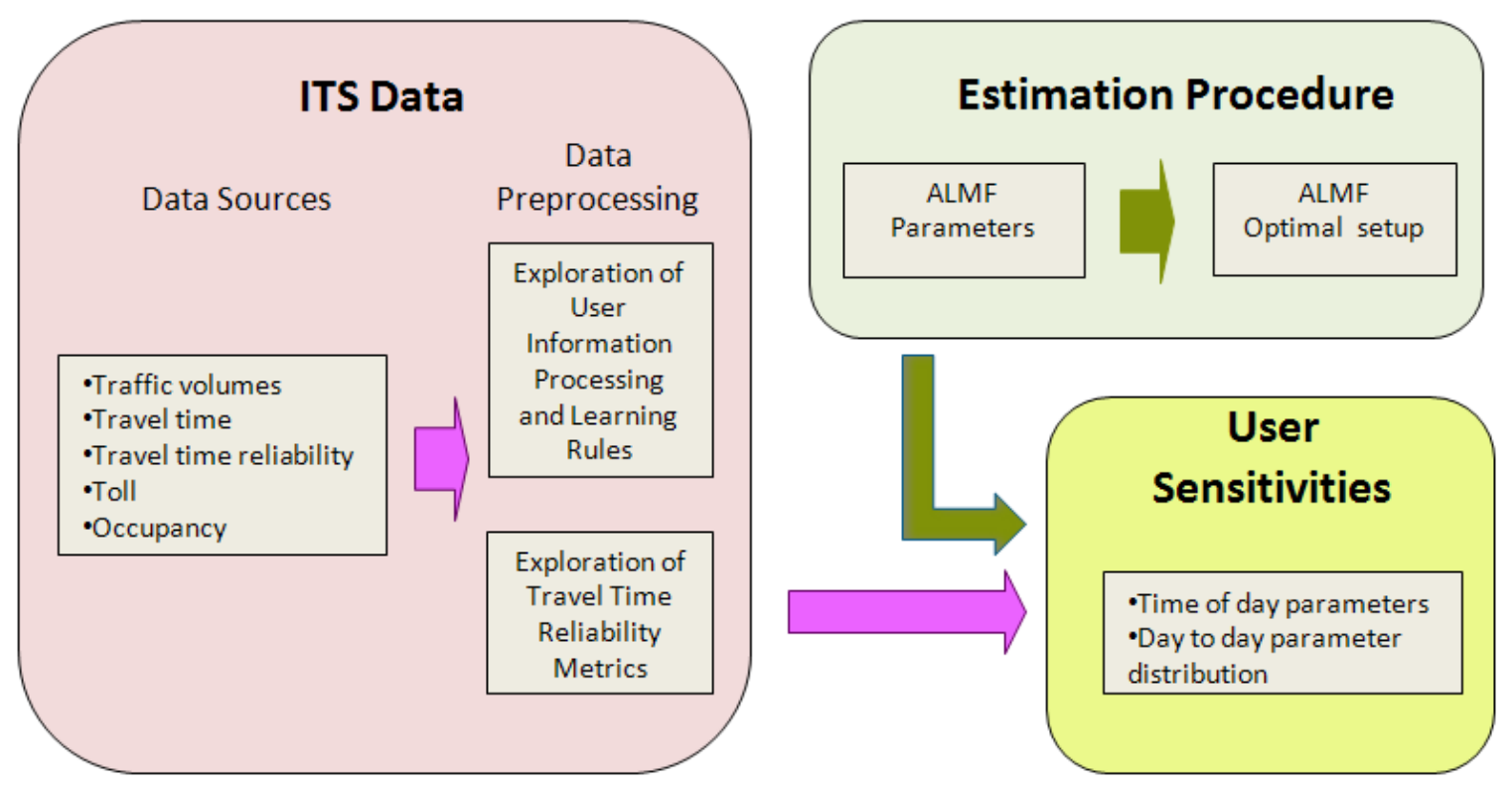

Figure 4.1: Estimation Framework

\subsection{Learning and Processing Information for a Facility}

Even though the initial motivation for the system performance perception and driver information processing described above was intended to be applied to individuals, still it is possible to use the same approach to approximate the characteristics of the user perception and user information processing when aggregated choices are observed on the facility. In particular it is possible to study the correlation between the observed proportion of users of the HOT lanes and the system performance measures considering different treatments of the performance measures. The hypothesis behind this is that different treatments of the system performance measures will correlate differently (better or worse) with the observed proportion of HOT lane users, and thus a better correlation would indicate that the information treatment better approximates the actual processes used on average by the system users. In order to proceed with the analysis, the following assumptions should be made clear: 
(a) It is assumed that the time period when commuters pass through the decision point does not change significantly from day-to-day, and thus the performance measures archived from previous days are representative of the conditions expected by commuters at that period of time. This assumption is somewhat reasonable given the characteristics of daily commuting in this corridor where the time of the day traffic pattern does not change significantly from day to day.

(b) Another assumption has to do with the fact that the archived performance measures correspond to the estimates provided by TMC software and thus they may or may not correspond to the actual system performance as experienced or perceived by the users.

(c) The fact that what is being correlated is the observed proportion of users and a measure of the performance difference between the HOT and GP lanes, implies that the users have perfect knowledge about the system performance in both lanes. This assumption should be carefully considered because users who systematically repeat the same choice daily may actually have a biased perception of the traffic conditions on alternative routes, and consequently a biased decision. 
Having these considerations in mind, this study focuses on the exploration of the following questions regarding driver information processing:

(a) What period of time is considered by users when assessing the historical performance of the system?

(b) Do the drivers have a fading memory? Or in other words, do drivers give more weight to more recent experiences or all experiences in their memory weight the same?

(c) Is there any evidence indicating that real time information has any impact on the observed proportion of the lane system users?

(d) And finally, is there any evidence indicating that travelers remember the most critical experiences only?

In order to assess these questions, a set of days was selected randomly from the dataset. For each of these days, the correlation between the proportion of HOT lane users and the system performance was assessed by time of the day, considering different treatments for the system performance data. A summary of the parameters used in the data processing is shown in Table 4.1 . 
Table 4.1: Data Processing Specifications for Learning and Information Processing

\begin{tabular}{|l|l|l|l|}
\hline $\begin{array}{l}\text { Information } \\
\text { processing } \\
\text { feature }\end{array}$ & $\begin{array}{l}\text { Dependent } \\
\text { Variable }\end{array}$ & $\begin{array}{l}\text { Independent } \\
\text { Variable }\end{array}$ & Data processing parameters \\
\hline $\begin{array}{l}\text { length } \\
\text { Memory }\end{array}$ & $\begin{array}{l}\text { Proportion of HOT } \\
\text { users }\end{array}$ & $\begin{array}{l}\text { Travel time } \\
\text { difference between } \\
\text { GP and HOT }\end{array}$ & $\begin{array}{l}\text { Travel time differences are estimated as the average } \\
\text { travel time considering different dataset lengths. } \\
\text { Myopic (day before), 5 days, 15 days, 30 days, 60 } \\
\text { days and 90 days datasets are used. }\end{array}$ \\
\hline $\begin{array}{l}\text { Fading } \\
\text { memory }\end{array}$ & $\begin{array}{l}\text { Proportion of HOT } \\
\text { users }\end{array}$ & $\begin{array}{l}\text { Travel time } \\
\text { difference between } \\
\text { GP and HOT }\end{array}$ & $\begin{array}{l}\text { Travel time differences are estimated as the weighted } \\
\text { average travel time considering a 60 day dataset } \\
\text { length. Three exponentially decreasing weigh rules } \\
\text { are used: b=1 (simple average), b=1.05 and b=1.15 }\end{array}$ \\
\hline $\begin{array}{l}\text { Real } \\
\text { information }\end{array}$ & $\begin{array}{l}\text { Proportion of HOT } \\
\text { users }\end{array}$ & $\begin{array}{l}\text { Travel time } \\
\text { difference between } \\
\text { GP and HOT } \\
\text { travel time differences are computed as the weighted } \\
\text { dataset simple average and the current estimate } \\
\text { provided by the TMC software. Five weights are } \\
\text { explored: a=1(no consideration of the real time } \\
\text { information), a=0.75, a=0.50, a=0.25 and a=0 (only } \\
\text { real time information is considered) }\end{array}$ \\
\hline $\begin{array}{l}\text { Gestalt } \\
\text { characteristics } \\
\text { of the travel } \\
\text { time }\end{array}$ & $\begin{array}{l}\text { Proportion of HOT } \\
\text { users }\end{array}$ & $\begin{array}{l}\text { Travel time } \\
\text { difference between } \\
\text { GP and HOT }\end{array}$ & $\begin{array}{l}\text { Travel time differences are computed as the average } \\
\text { of the worst 1, 5, 10 and 20 days out of 60days. }\end{array}$ \\
\hline
\end{tabular}

\subsubsection{Memory Length}

As described in section 4.1, the objective of this exploratory exercise is to determine the period of time that provides the best correlation between the proportion of HOT users and the differences in travel time between the HOT and the GP lanes. The HOT and GP travel time estimates have been computed considering different periods of time; namely, 1 day (myopic approach), 5, 10, 15, 30, 60, and 90 days. Figures 4.2 and 4.3 show the relationships between the proportion of HOT users and the difference in travel time for the AM and PM peaks respectively. The figures depict the results observed for a typical day randomly chosen from the database, although similar patterns are observed for most days in the database. From the figures, in both cases it is observed that using fewer days (5 days) to estimate the travel times barely depicts any relationship between the variables considered, especially in the AM peak where it is known that traffic patterns are more variable. Conversely, it is observed that using a longer period of time (60 days) shows in both cases (AM and PM) a stronger pattern, which at the same time is consistent with the 
intuition that the bigger the difference in travel time, the higher the proportion of HOT users. In order to assess the degree of correlation between the proportion of HOT users and the difference in travel time, the coefficient of correlation $\left(R^{2}\right)$ was estimated for the dataset. The results are shown in Figure 4.4 for the AM and PM peak periods; in general, it is observed that the longer the period of time considered, the larger is the coefficient of correlation. However, no further increments are observed beyond 60 days where the coefficient of correlation remains constant. The latter may suggest a threshold for the maximum number of days that are sufficient for estimating travel time on this particular facility.

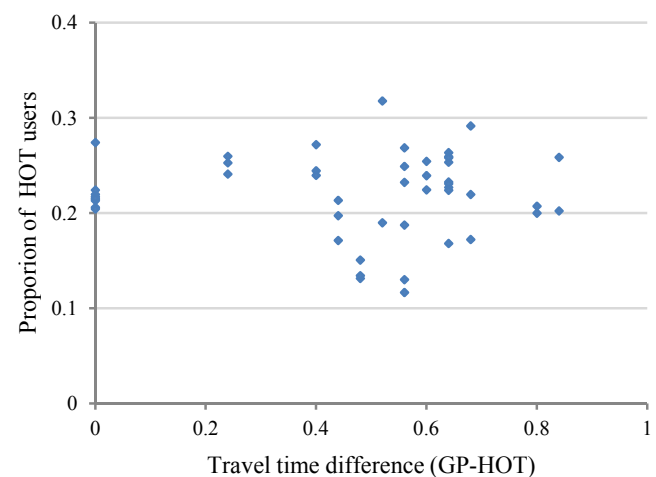

(a) 5 days

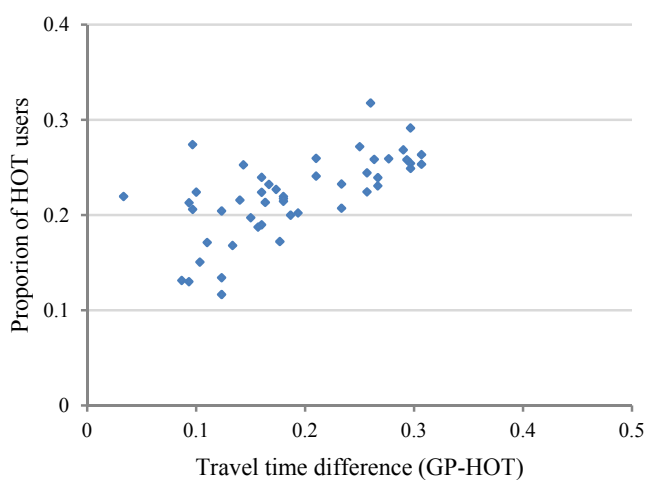

(b) 60 days

Figure 4.2: AM Travel Time Adjustment Considering 5 and 60 Days Respectively

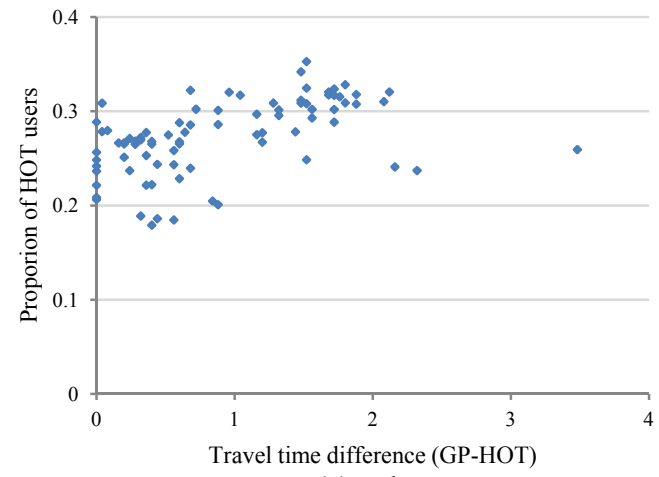

(a) 5 days

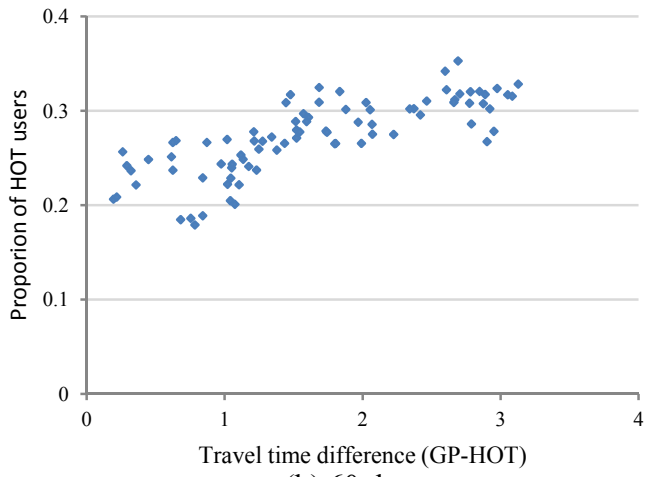

(b) 60 days

Figure 4.3: PM Travel Time Adjustment Considering 5 and 60 Days Respectively 


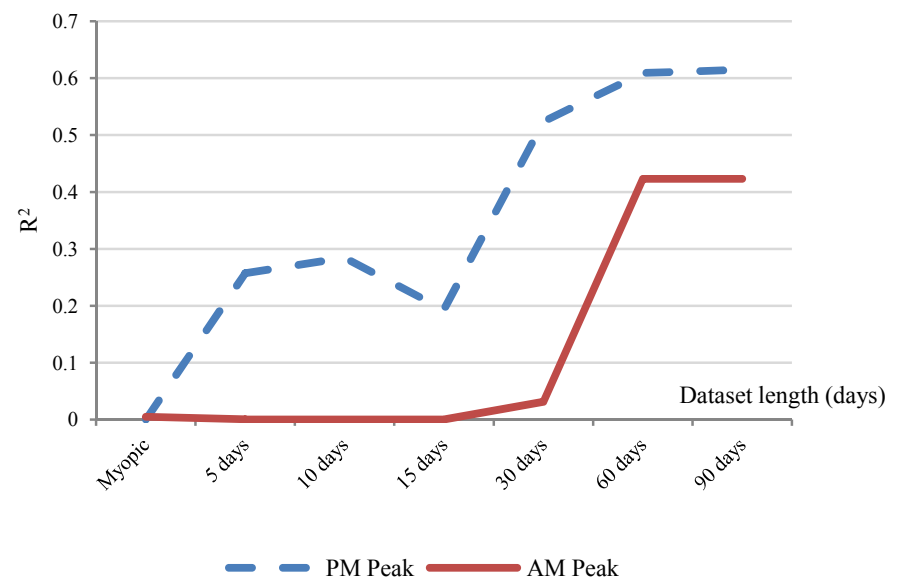

Figure 4.4: $\boldsymbol{R}^{2}$ considering different dataset lengths in AM and PM periods

\subsubsection{Fading Memory}

In addition to the size of the dataset that the users may consider when assessing the performance of the facility, the question about the weight of each record in the final assessment is addressed. For this purpose, different exponentially decreasing weights are considered to affect the historical differences in travel time between HOT and GP lanes. Figure 4.5 shows the exponentially decreasing weights used for 60 days of measured travel time data. As shown in the chart, using $b=1.0$ represents the case where all the records receive the same weight; this is the simple arithmetic mean of the sample. On the other hand, using $b=1.05$ corresponds to a case where the latest experiences are somewhat more important than the older ones in the final travel time assessment. Finally, using $b=1.15$ is conceptually similar to using $b=1.05$; but comparatively speaking, in the latter case, older experiences fade faster than the former case. 


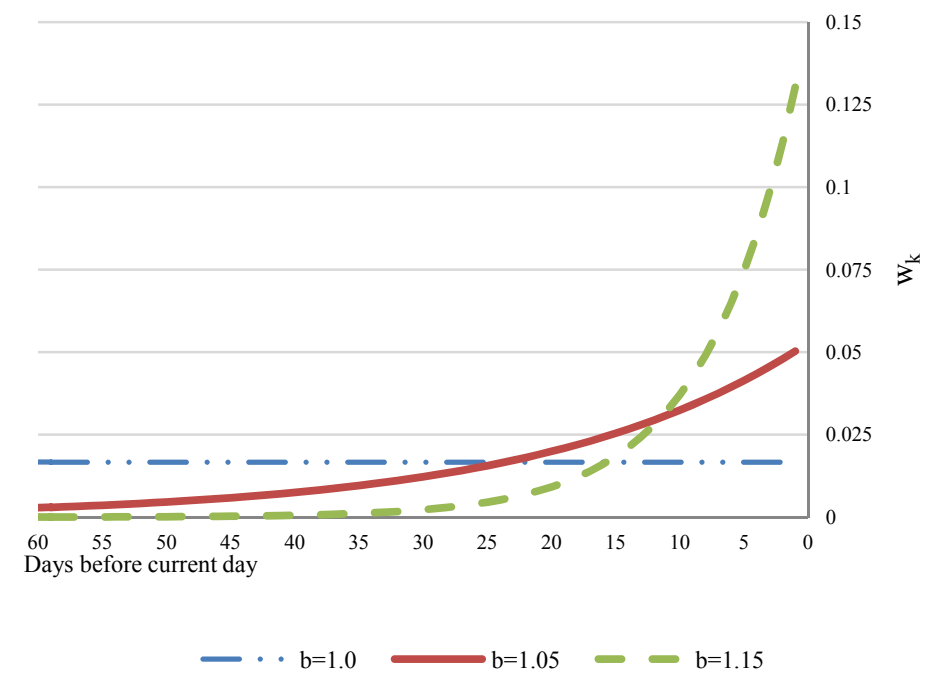

Figure 4.5: Exponentially Decreasing Values of $w_{k}$ for Different Values of $b$

Figures 4.6 and 4.7 show the relationship between the proportion of HOT users and the difference in travel time for the AM and PM peaks respectively when different fading rules are used. In both cases, using fading rules do not help to better visualize the relationship between the proportion of HOT users and the travel time differences. This is consistent with the results found in the previous section where in order to obtain well defined patterns between the proportion of HOT users and differences in travel time, a period of 60 days was found to be the best time inreval. In this sense, using fading memory rules is equivalent to shorten the period of time considered to collect the travel time samples. Thus, considering fading rules do not help correlating better the observed proportion of HOT users and the system performance. 


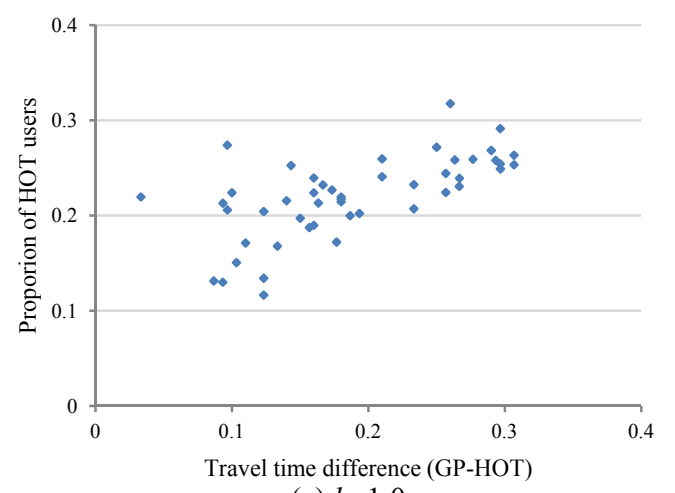

(a) $b=1.0$

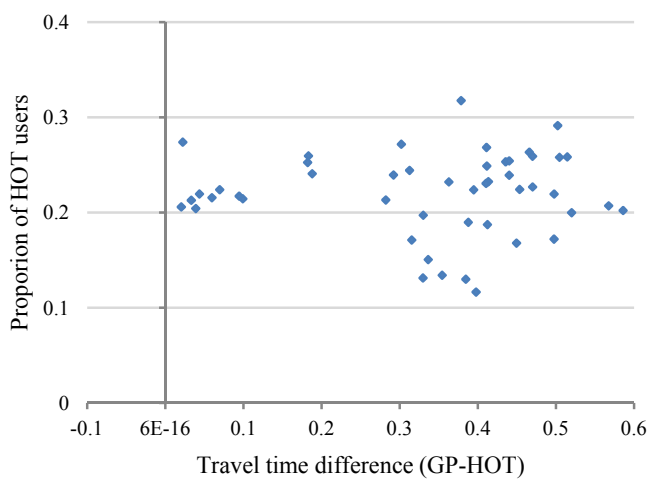

(b) $b=1.15$

Figure 4.6: AM Travel Time Adjustment Considering $b=1.0$ and $b=1.15$ Respectively

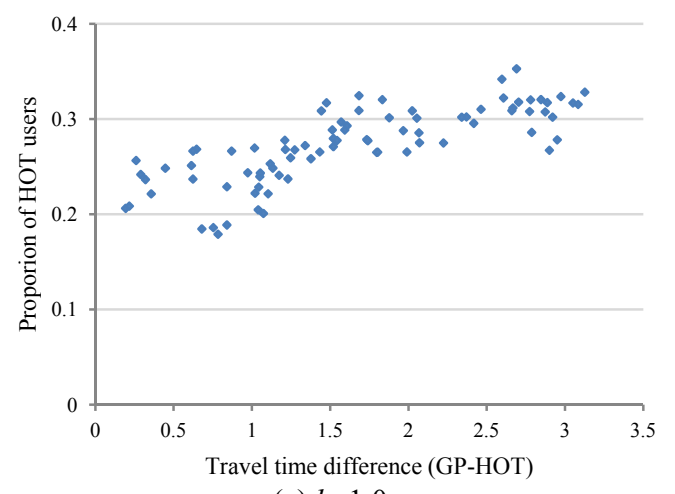

(a) $b=1.0$

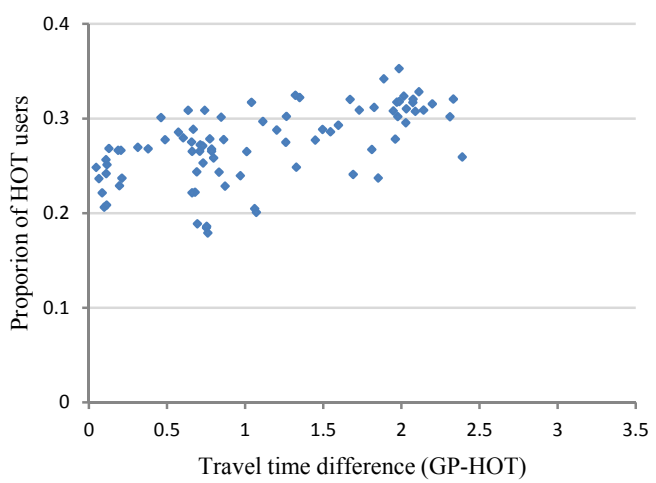

(b) $b=1.15$

Figure 4.7: PM Travel Time Adjustment Considering $b=1.0$ and $b=1.15$ Respectively

The exploration of the correlation coefficient between the proportion of HOT users and the differences in travel time further support the fact that the simple average better represents the process used by individuals to assess the travel time differences. Figure 4.8 shows the correlation coefficient between the proportion of HOT users and the travel time differences for various values of the parameter $b$. From the figure is clear that using fading memory rules tends to dilute the relationship between these quantities. 


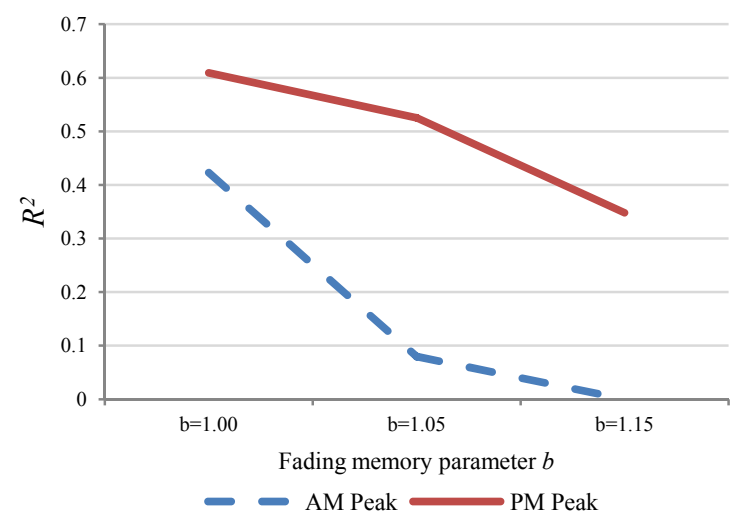

\section{Figure 4.8: $R^{2}$ Considering Different Fading Memory Rules}

\subsubsection{Real Time Information Impact}

The impact of real time information on the observed traffic patterns is also explored. In South Florida, besides the information the driver can collect from his environment while he is driving, there are a number of real time sources of information that can eventually be taken into account in the traveler's decision making process. These sources of information include Dynamic Message Signs (DMS) with actual travel time estimates, local radio broadcasts with general traffic information, a 511 system with facilityspecific traffic information and other more informal sources of information, like friendly advice by telephone, and so on. In addition, the pricing of the HOT lanes may give motorists a hint of the levels of expected congestion; although in reality, these prices are not set based on the difference in travel time but on managed lanes Level of Service (LOS). In this study, several hypothetical cases are explored ranging from utilizing only real time information $(a=0.0)$ to utilizing only historic information $(a=1.0)$. In all of the cases explored, the historical information is based on the mean travel time computed over 60 days of data. Figure 4.9 and Figure 4.10 show the relationship between the proportion of HOT users and the difference in travel time for the AM and PM periods respectively. 
The figures show the two extreme values of the real time and historical information weights used. These results are quite interesting, particularly for the AM period where relying exclusively on real time information actually provides some negative numbers for the difference in travel time between HOT and GP lanes. This result indicates that if users were basing their decisions only on real time information, the observed behavior would be the in contradiction to the expected one. In other words, drivers would be paying for the use of the HOT lanes while actually the GP lanes have a lower travel time and consequently the proportion of HOT users should be zero. As the use of the real time information is reduced to zero, it is observed that the expected behavior emerges in both periods.

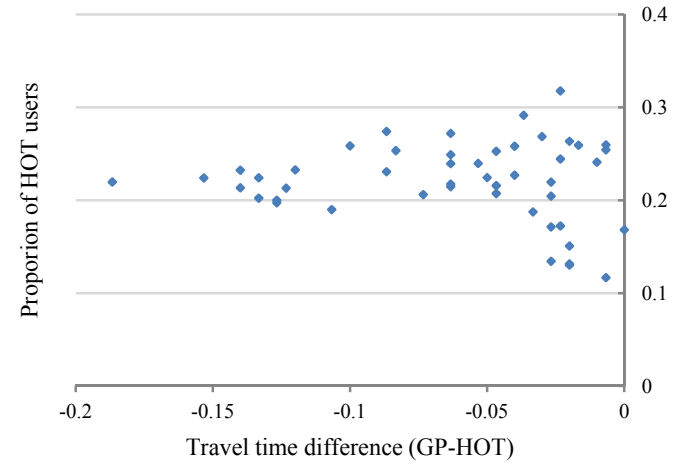

(a) $a=0.0$

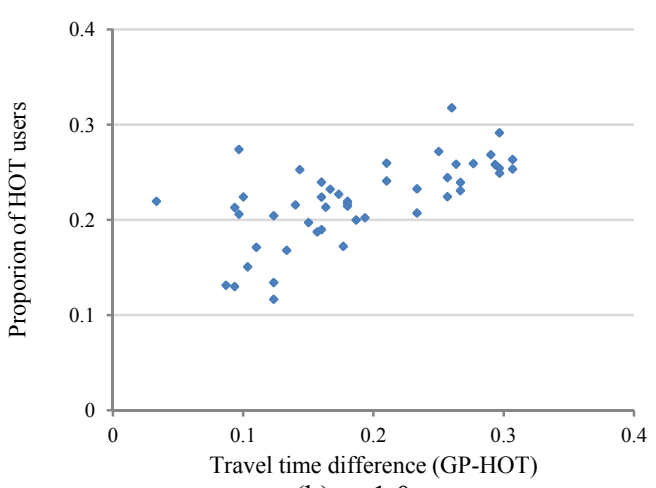

(b) $a=1.0$

Figure 4.9: AM Travel Time Adjustment Considering $a=0.0$ and $a=1.0$

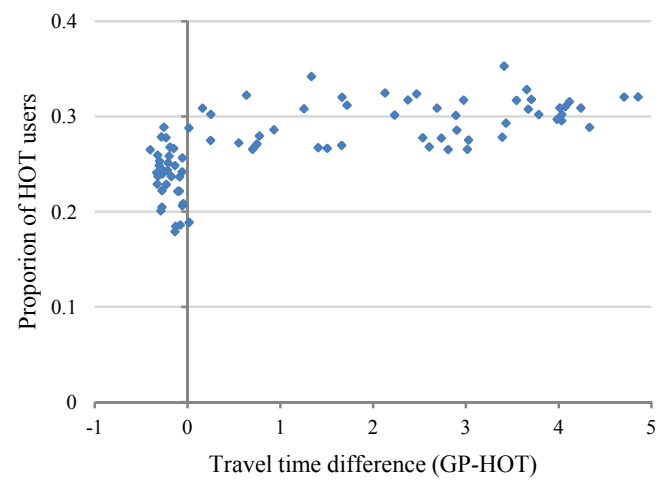

(a) $a=0.0$

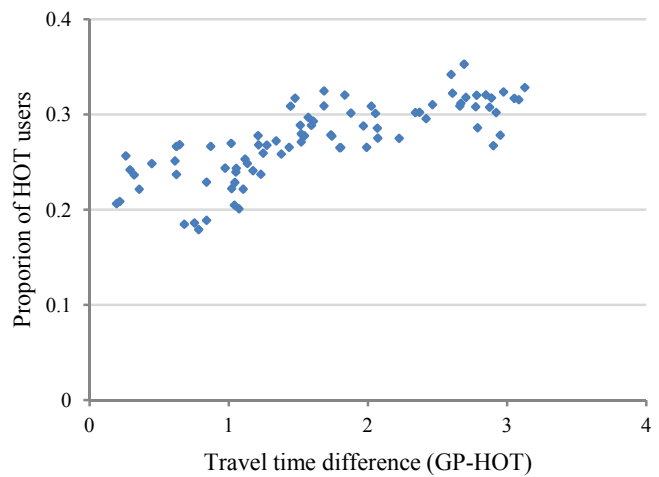

(b) $a=1.0$

Figure 4.10: PM Travel Time Adjustment Considering $a=0.0$ and $a=1.0$ 
Figure 4.11 depicts $R^{2}$ the between the observed proportion of HOT users and the travel time difference between GP and HOT lanes. It can be observed that there is a relationship between the coefficient of correlation and the weight considered for the real time information. In particular a better correlation between the quantities of interest is obtained when real time information is minimal or not considered at all. This possibly reflects the fact that, for the explored conditions, the actual difference in travel time between the GP and HOT lanes is not normally communicated to travelers.

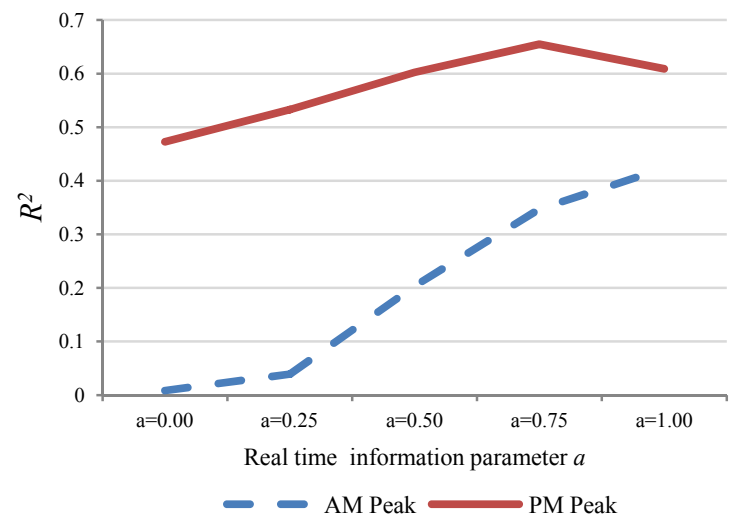

Figure 4.11: $\boldsymbol{R}^{2}$ Considering Different Real Time Information Parameter $a$ 4.1.4. Gestalt Characteristics of the Travel Time

This section explores the possibility that travelers assess their travel time based on a subset of their experienced travel times. In particular, the subsets considered are defined by the average of the worst travel time experiences in a given number of days. The logic behind this is exploration resides in the fact that some individuals may remember only those events that are significantly distinguishable from the average conditions and then having the potential to be particularly influential. In this study four cases were studied: travel time differences between the GP and HOT lanes is computed as the average of the 20 worst days in 60 days, 10 worst days in 60 days, 5 worst days in 60 days and the 
single worse day in 60 days. Figure 4.12 shows the results for each case respectively. It can be observed that considering the average of the worst days tend to increase the range for the differences in travel time while the range of the proportion of HOT users remain the same. In other words, considering Gestalt characteristics of the travel time makes the proportion of HOT users less sensitive to changes in the travel time differences and in consequence the travel time variable losses its explanatory power. Thus, it can be concluded that there is no evidence that considering Gestalt characteristics of the travel time can explain better the observed traveler choices.

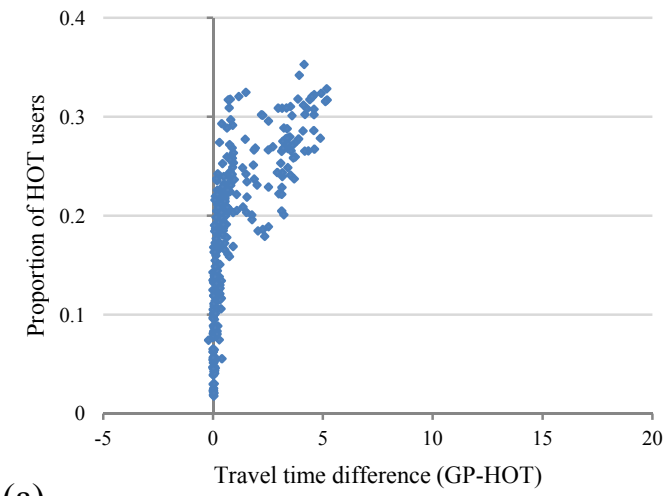

(a)

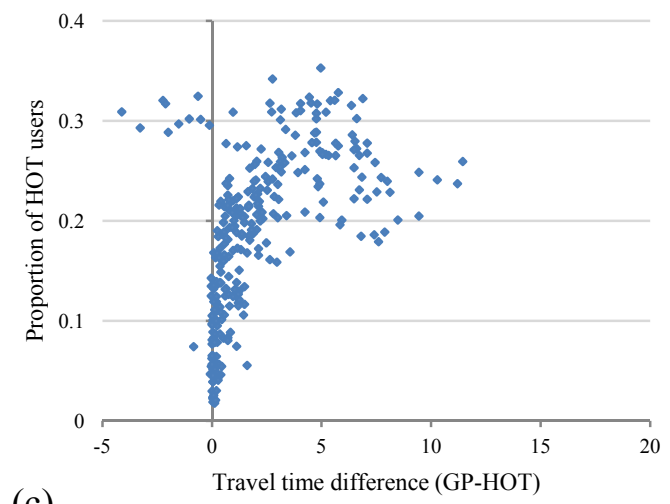

(c)

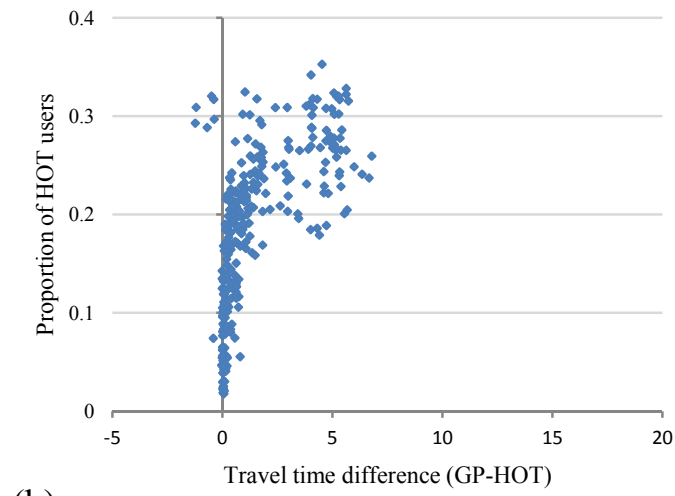

(b)

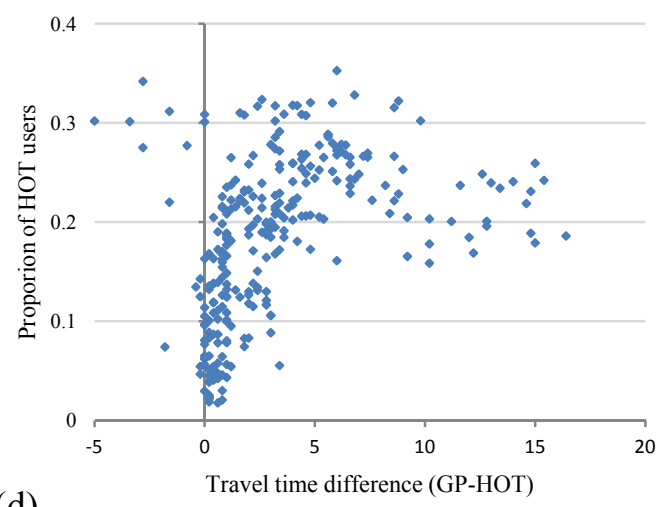

(d)

Figure 4.12: Relationship Between the Proportion of HOT Users and the Gestalt Characteristics of the Travel Time, (a)20/60, (b)10/60, (c)5/60 and (d)1/60 


\subsubsection{Model for Information Processing and Learning Summary}

This section has explored key aspects of the driver behavior with respect to the perceived system performance. These aspects are related to the length of memory to assess traffic conditions, fading rules to favor latest/oldest experiences, and the role of real time information that may be considered by the driver. The exploration has been carried out using only aggregate observations of driver choices in a system composed of a GP and HOT lanes, and consequently the validity of the conclusion is subject to the validity of the assumptions considered in the study. In general, it is possible to conclude that individuals using this facility tend to assess their choices based on the average travel time estimated from their own experiences; that is, they give the same weight to all experiences within the time window considered. In addition, it is noticed that this assessment of the travel time is performed with limited use of real-time information that may be broadcast in real time by different means. Finally, it was found that there is no evidence that considering Gestalt characteristics of the travel time can explain better the observed traveler choices.

\subsection{Travel Time Reliability Metrics}

It was mentioned that the traveler's decision-making process may be influenced by the level of consistency of travel conditions over time. This section addresses the question of how to incorporate such travel time consistency by means of reviewing how travel time distributions change by time of day at fine temporal aggregation levels, the sensitivity of various travel time reliability metrics to these variations, the effect on the aggregation level choices of the calculated metrics, and the amount of data required to estimate stable 
values of the reliability metrics. The investigation is made for a facility that has GP lanes and HOT lanes.

In this section the variation of the parameters of travel time distributions by time of day, the sensitivity of various reliability metrics to these variations, the effect of time of day analysis interval on the calculated metrics, and the amount of data required to estimate stable values of the reliability metrics is investigated. The investigation is made for a facility that has GP lanes and HOT lanes, allowing the comparison of how travel time reliability attributes between the two facilities by time-of-day can be depicted by different reliability metrics. The study also explores the trends of the variations of various metrics as the congestion increases during the peak period, which is important when selecting reliability metrics for various applications such as the use of the metrics as part of the generalized cost functions of assignment models and in optimization of strategies such as congestion pricing.

\subsubsection{Utilized Travel Time Data}

Agencies have used Intelligent Transportation Systems (ITS) devices to collect traffic parameter measurements for operational purposes. In recent years, these agencies have started archiving the collected information for future uses (FHWA, 2004). The availability of the archived information allows the analysis of travel time distributions

and the associated reliability measures at a fine time scale compatible with the requirements of advanced strategies and analysis applications. 
The data used in the analysis was obtained from a 6.5 mile segment of the northbound I-95 limited- access facility in Miami, Florida. This segment has a total of six lanes, two of which are HOT lanes and the remaining four lanes operating free of charge as GP lanes. The two HOT lanes have been in operation since December 2008, utilizing a dynamic congestion pricing scheme. Registered vehicles with high occupancy can use the HOT lanes without paying tolls. The HOT lanes have a single entry point and a single exit point and are fully segregated from the GP lanes by plastic poles. This section of I-95 is equipped with point traffic detectors located every 0.3-0.5 miles that collect volume, speed, and occupancy measurements every 20 seconds for both the HOT and GP lanes. The corridor operations and the data gathered by ITS devices are managed by the Florida Department of Transportation (FDOT) District 6 by means of traffic management software referred to as the SunGuide software (SWRI, 2010).

This study utilizes travel time estimates for the HOT and GP lanes that are archived for every minute of the day by the SunGuide software. The travel time is estimated by the system based on the speed measurements collected by the microwave detectors installed on the corridor utilizing the mid-point speed estimation method. The mid-point method is a widely used travel time estimation algorithm in traffic management center software. The mid-point method assumes that each detector speed measurement represents the speeds of half distances to the next detector on both sides. The segment travel time is thus calculated as follows: 


$$
T T_{1-2}=\frac{L_{1-2} / 2}{S_{1}}+\frac{L_{1-2} / 2}{S_{2}}
$$

where $T_{1-2}$ and $L_{1-2}$ are the travel time and length of the link between detectors 1 and 2, respectively. $\quad S_{1}$ and $S_{2}$ are the speeds measured by detectors 1 and 2 , respectively.

One year's worth of data was used in the analysis. However, also using data collected for shorter periods of time is investigated, as described later in this section. Only weekday travel time data was used in the analysis.

\subsubsection{Time-Variant Travel Time Distributions}

Travel time reliability metrics are calculated to reflect the variability of travel time from day to day. Thus, they are supposed to represent the attributes of travel time distributions that are most relevant to the perception of system reliability. Since the purpose of this study is to examine the attributes of time-variant reliability metrics, it is useful to examine first the time variant parameters of travel time distributions of the HOT and GP lanes. In addition, as stated earlier, previous reliability studies have calculated reliability measures for the whole peak periods, implicitly assuming that the travel time

distribution parameters remain constant during these periods of the analysis. Investigating the variations in travel time distribution parameters by time interval allows the examination of the validity of this implicit assumption.

Comparing the parameters of travel time distributions for GP and HOT lanes under different conditions can also provide important information regarding the sources of the unreliability of these facilities. These sources can be classified as recurrent and non-recurrent events. Recurrent events include demand fluctuations, variations in 
traveler behaviors, and stochastic variations in flow breakdown and capacity. Non-recurrent events include incidents, work zones, special events, and weather events. It is logical to expect travel time variability mainly due to recurrent events to have distributions that are much flatter and more symmetrical compared to conditions with variability significantly influenced by non-recurrent events, which are expected to have skewed distributions. However, the shapes of the distributions could also be influenced by facility types and their operational characteristics. Previous studies have produced limited information regarding the characteristics of travel time distributions during incident and no-incident conditions and their influences on travel time variability, as explained next.

Li (Li et al., 2006) found that, under free flow conditions of a transportation system, travel time distribution has the shortest right tail whereas the afternoon peak has the most skewed distribution. They also found that the variation in demand in the morning peak explains most of the travel time variability while incidents play a major role explaining the variability during the PM peak. The authors warned that these results are likely to be site specific. Boyles (Boyles et al., 2010) examined four operational conditions on two corridors: no incident-good weather (NIGW), poor weather (PW) and incident present (IP). They found that the normal distribution is best suited for describing speed in NIGW conditions, while the beta distribution is best suited for fitting PW and IP conditions. The authors also warned analysts about the site specific character of the results. Tu (Tu et al., 2008) pointed out that the effect of traffic incidents on travel time distribution is a function of the volume using the facility. Below a certain threshold, (1400 vphl) incidents slightly increase the $10^{\text {th }}$ percentile of travel time by $3 \%$ and the 
median travel time by an average of $6 \%$. Above this threshold, incidents result in significantly higher median travel time ( $38 \%$ increment) while the $90^{\text {th }}$ percentile travel time is increased in average by $75 \%$.

This study compares the parameters of the travel time distributions, namely: the median, standard deviation, skewness, and kurtosis (NIST/SEMATECH, 2011). The median travel time is a measure of central tendency that is preferred to the mean in the case of skewed distributions (Cambridge Systematics, 2010; Rakha et al., 2007; Pu, 2010). The standard deviation is a measure of the travel time variability and has been used as a reliability measure by itself. The coefficient of variation, which is the standard deviation divided by the mean, has also been used for this purpose (Rakha et al., 2007). Skewness is a measure of travel time distribution asymmetry and its value can be negative or positive with positive values indicating that a longer tail of the distribution is on the right hand side of the distribution and the bulk of the travel time values lie to the left of the mean and the median. The fourth parameter, the kurtosis, is a measure of the "peakedness" of the travel time distribution. Kurtosis combined with skewness can be used together to assess how far is the travel time distribution from the symmetrical normal distribution with the same median and standard deviation.

Figure 4.13 summarize the results for the median, standard deviation, skewness and kurtosis. Travel times have been computed every 5 minutes, and it is observed that the HOT lanes had a lower median travel time than had the GP lanes. Both facilities reached their maximum median travel time at 5:00 PM, which is 8 minutes for the HOT lanes and 12 minutes for GP lanes (the free flow travel time is about 6 minutes). Figure 4.13 also shows that as the median travel time increases, so does the standard deviation. 
However, the skewness and kurtosis are higher at lower congestion levels, indicating lower correspondences between the distributions of travel time and normal distributions at low congestion levels and possibly a higher contribution of non-recurrent events to travel time variability.

Also, Figure 4.13 clearly indicates that the shape of travel time distributions is highly variable as a function of time. This is even more certain in the case of managed lanes due to the shorter peak period that occurs only after the GP lanes start getting congested and motorists start diverting to HOT lanes due to this congestion. During most of the investigated period, the travel time variability of the HOT lanes as measured by the standard deviation is lower than that of the GP lanes, except at the peak hour (5:00 PM), when the variability of both facilities approaches each other. This occurs in spite of the lower travel time median on the HOT lanes at this peak hour.

The variability of travel time in the HOT lane and the higher skewness and kurtosis of the travel times distribution of the HOT lanes compared to GP lanes during the peak period could be attributed to a higher contribution of the non-recurrent events to the unreliability in the HOT lanes, higher demand variations on the HOT lanes from day to day compared to GP lanes, and/or higher sensitivity of the HOT lane travel time to demand variations. The contributions of these three factors to travel time variability were investigated in this study. First, to determine the contribution of non-recurrent congestion, the days with either incidents in the HOT or GP lanes were filtered out of the analysis dataset. It was observed that removing the days with incidents does not change significantly the travel time distribution (less than $5 \%$ per bin) leading to the conclusion that the non-recurrent events are not the main factor that explain the increase of the 
travel time distribution skewness. It was further determined that the standard deviations of demands of the HOT and GP lanes are close to each other indicating that this factor is also not the main reason for the high variability of the travel time of the HOT lanes around the peak period. Further examination of the data indicates that the main reason for the high variability of the HOT lanes is the lower capacity of the HOT lanes compared to GP lanes, which causes a higher sensitivity of the travel time to the increase in demand. Based on the collected data, the capacity of the managed lanes is about $1,300 \mathrm{veh} / \mathrm{hr} / \mathrm{lane}$, and the capacity of GP lanes is about $1,900 \mathrm{veh} / \mathrm{hr} / \mathrm{lane}$.
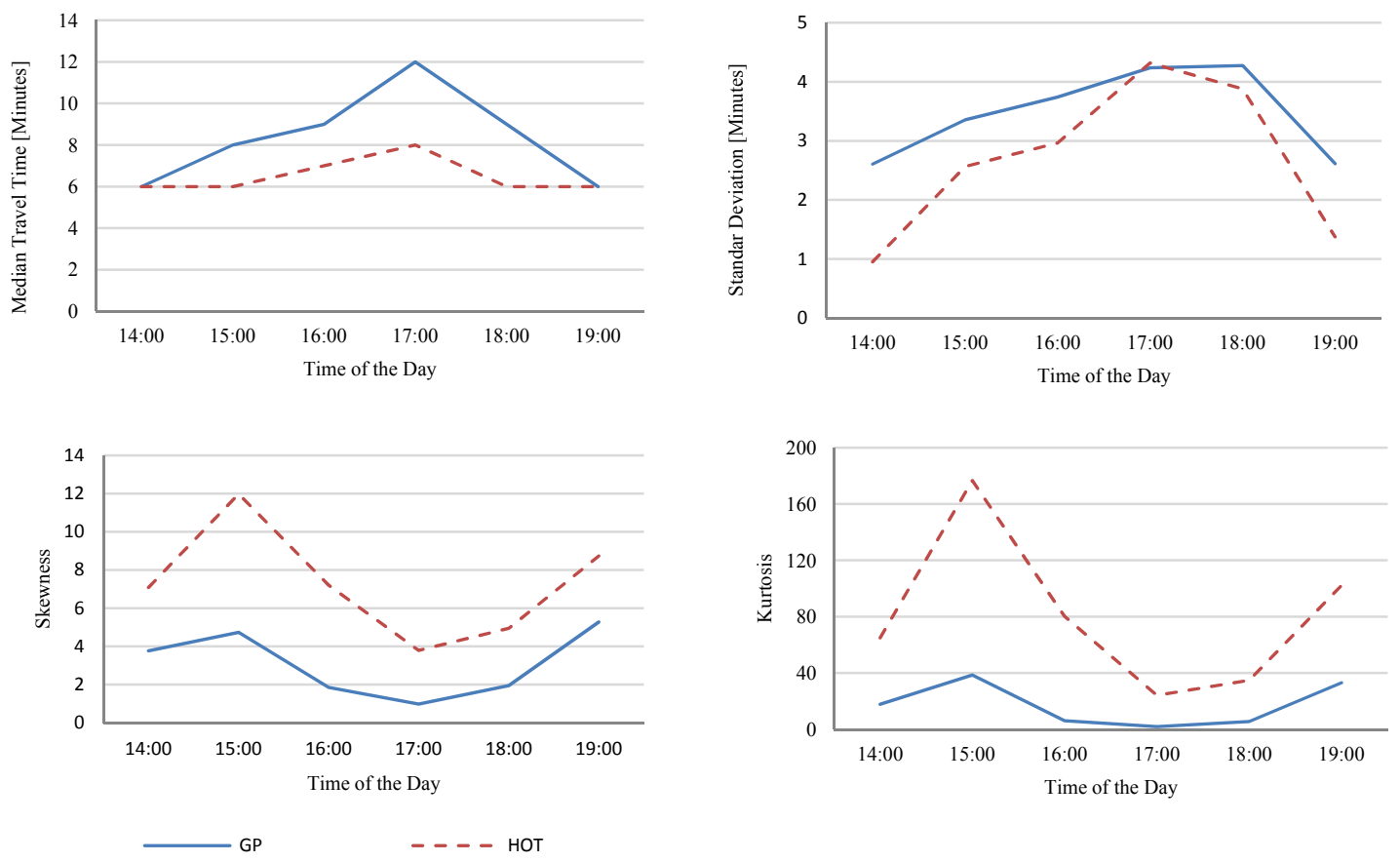

Figure 4.13: Travel Time Distribution Shape Parameters

\subsubsection{Time-Variant Reliability Metrics}

As described previously, it is also important to examine how the travel time reliability metrics vary by time-of-day and particularly how do they react to the increase in demand. These metrics necessarily have to be sensitive to the increase in congestion and they need 
to show a consistent trend as the congestion increases in order to allow their use in highway capacity/traffic analysis applications, or when included as parameters in the objective functions of dynamic traffic assignment tools or of the optimization of the pricing of managed lanes and other strategies.

There are a number of metrics that have been used to quantify reliability. The variation in the parameters of the travel time distributions with the increase in congestion by time of the day as described in the previous section is expected to have significant impacts on the values of these metrics. In this study, an investigation was made of the sensitivity of the reliability metrics to the changes in the congestion levels and the ability of these metrics to reflect the reliability differences between the HOT and GP lanes in a time-variant context. Table 4.2 shows the definitions of travel time reliability metrics used in this study. These measures are mainly selected based on the recommendations given in SHRP2 L03 project (Cambridge Systematics, 2010) and constitute a common set of metrics used for reliability assessments.

Table 4.2: Travel Time Reliability Operational Definitions

\begin{tabular}{|l|l|}
\hline $\begin{array}{l}\text { Reliability Performance } \\
\text { Metric }\end{array}$ & Definition \\
\hline Buffer Index (BI) & $\begin{array}{l}\text { The difference between the } 95^{\text {th }} \text { percentile travel time and } \\
\text { the average travel time, normalized by the average travel } \\
\text { time. }\end{array}$ \\
\hline $\begin{array}{l}\text { Failure/On-Time } \\
\text { Performance }\end{array}$ & $\begin{array}{c}\text { Percent of trips with travel times less than: } \\
\bullet \quad 1.1^{*} \text { median travel time } \\
1.25^{*} \text { median travel time }\end{array}$ \\
\hline $95^{\text {th }}$ Planning Time Index & $95^{\text {th }}$ percentile of the travel time index distribution \\
\hline $\begin{array}{l}80^{\text {th }} \text { Percentile Travel Time } \\
\text { Index }\end{array}$ & $80^{\text {th }}$ percentile of the travel time index distribution \\
\hline Skew Statistics & $\begin{array}{l}\text { The ratio of } 90^{\text {th }} \text { percentile travel time minus the median } \\
\text { travel time divided by the median travel time minus the } 10^{\text {th }} \\
\text { travel time percentile }\end{array}$ \\
\hline Misery Index & $\begin{array}{l}\text { The average of the highest five percent of travel times } \\
\text { divided by the free-flow travel time. }\end{array}$ \\
\hline
\end{tabular}


In this study, the reliability metrics in Table 4.2 were estimated for the GP and HOT lanes, utilizing different time interval lengths in the analysis. The results presented in Figures 4.14 and 4.15 are based on 15 minute intervals. Figure 4.14 and Figure 4.15 show different trends of different reliability metrics with time of day for the GP and HOT lanes respectively, as explained below.

The metrics that exhibit continuity and sensitivity in their variations in response to the increase in variability as the congestion in the peak hour is approached are the $95^{\text {th }}$ Percentile Planning Time Index (PTI), $80^{\text {th }}$ Percentile PTI, and the Misery Index. Figures 4.14 and 4.15 show that the use of the $95^{\text {th }}$ Percentile PTI metric and even more the use of the $80^{\text {th }}$ percentile PTI metric clearly indicate that the reliability decreases as the peak demand period, at 5:00 PM, approaches. The reliability is lowest at this peak reflecting the highest variability observed when examining the parameters of the travel time distributions, as discussed in the previous section. Figures 4.14 and 4.15 also show that the $95 \%$ PTI of the GP lanes is higher than that of the HOT lanes except at the peak hour, at which the PTI is equal for both facilities, reflecting the comparable travel time variability of the GP and HOT lanes at the peak hour. It is interesting to note that the $80^{\text {th }}$ percentile PTI is lower for the HOT lanes for all of the investigated hours, including the peak hour at 5:00 PM, and that the difference in reliability between the GP and HOT lanes is higher when the comparison is based on the $80^{\text {th }}$ percentile PTI compared to when measured based on the $95^{\text {th }}$ percentile PTI.

A similar trend to those observed with the PTI measurements discussed above was observed with the Misery Index, as indicated in Figures 4.14 and 4.15 For the GP and HOT lanes, the Misery Index values are lower in the uncongested periods compared to 
the congested periods, as expected. The Misery Index of the HOT lane is lower than that of the GP lanes prior to the peak hour but exceeds it at the peak hour.

The results of examining the other measures do not show the consistent trend of increase with the increase in congestion, observed when examining the PTI and the Misery Index in the discussion above. Figure 4.14 shows that the BI metric for the GP lane is not sensitive to the increase in congestion. BI is computed as the difference between the $95^{\text {th }}$ percentile and the mean divided by the mean travel time. The BI value of the GP lanes remains almost constant during the peak period because, although the variability of travel time increases significantly as the peak demand approaches the values of the numerator in the BI calculations, the travel time mean (or median) also increases as the congestion increases. For the GP lanes, the rate of change of the travel time mean/median parameter is close to the rate of change of the difference between the $95^{\text {th }}$ percentile and the mean/median travel time resulting in BI values that remain almost constant. In the case of the HOT lanes, the rate of increase in the variability of travel time with the increase in congestion is higher than the rate of the increase in the mean/median resulting in an increase in the BI. The inconsistency and lack of sensitivity of the BI to the increase in congestion in some cases may limit its use, at least for some reliability assessment and analyses tasks.

The Failure/On-Time metric (FOT) represents the number of occurrences of travel time that are lower than the median travel time times a factor (1.1 or 1.25$)$. The implication is that the higher this metric is, the more reliable the system is, because more of the travel time instances are close to the median. The value of this metric is affected by both the median and standard deviation values. As discussed previously, as the 
congestion increases, both the median and standard deviation increase. The results shown in Figures 4.14 and 4.15 indicate that there is no clear trend of the time-variant trend of FOT metric with the increased congestion, since the change in the metric value from one step to the next depends on how the rate of change in the median value is compared with the rate of the change in the standard deviation for the facility under consideration.

The trend of the Skew Statistic with the increase in congestion is also not very clear. It appears to be a function of the relative contributions of different sources of unreliability to the total travel time variability. For the GP lanes, it is clear that the value of this metric is lower at higher congestion levels indicating more symmetrical distributions due to the higher contribution of the recurrent congestion to the variability. The variations due to recurrent congestion have been characterized by a more symmetrical distributions (less skewed distributions), as described earlier. For HOT lanes, the Skewness Statistic initially increase as the shoulders of the peak hour are reached. As explained earlier in this paper, the travel time on the HOT lanes has a high sensitivity to the variations in demands due to the lower capacity of the managed lanes. This causes the variation in demands at the shoulder of the peak results in a high increase in the variability and Skewness Statistic. However, at the peak hour itself, the managed lanes operate consistently at capacity causing a lower variability between days and significantly lower Skewness Statistic. 


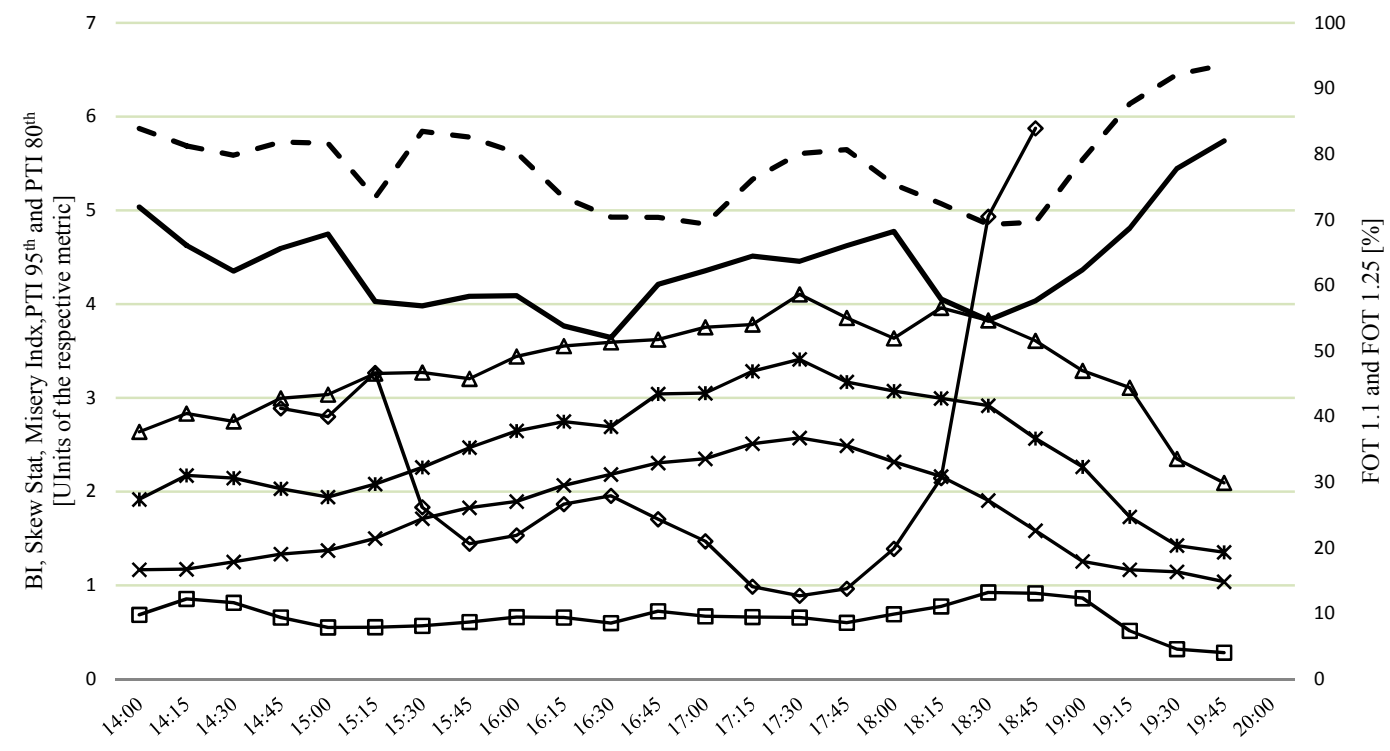

Time of the Day

$\longrightarrow$ BI $\longrightarrow$ PTI $80 \longrightarrow$ PTI $95 \longrightarrow$ SKEW STAT $\longrightarrow$ MISERY INDX $\longrightarrow$ FOT $1.1-$ - FOT 1.25

\section{Figure 4.14: Reliability Metrics Variation with Time-of-Day GP Lanes}

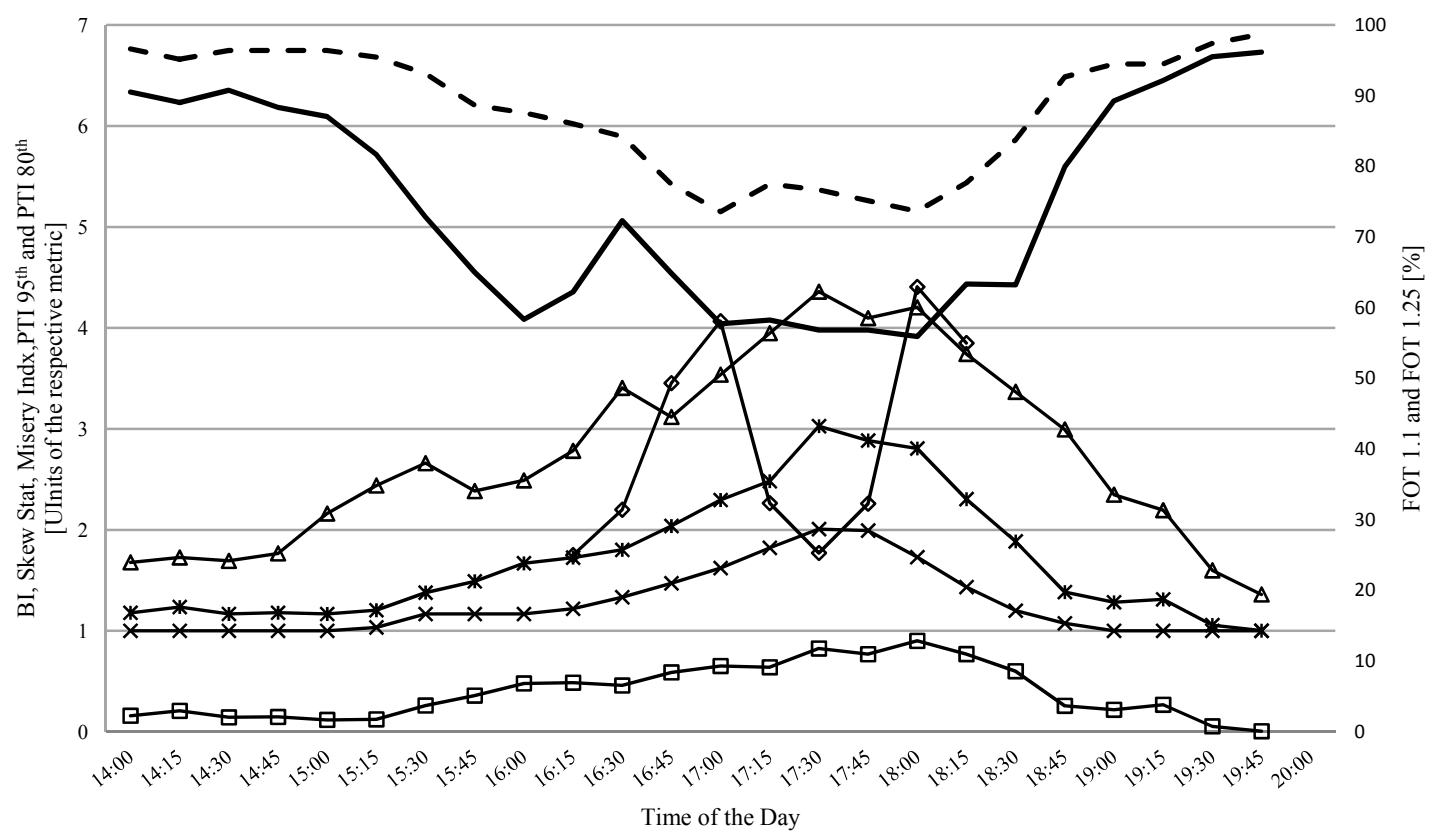

$\square$ BI $\longrightarrow$ PTI $80 \longrightarrow$ PTI $95 \multimap$ SKEW STAT $\longrightarrow$ MISERY INDX $\longrightarrow$ FOT $1.1-$ - FOT 1.25

Figure 4.15: Reliability Metrics Variation with Time-of-Day HOT Lanes 


\subsubsection{Analysis Period Length}

The discussion presented in the previous sections indicates that there is a considerable variation in the travel time distribution parameters and travel time reliability metrics when these parameters and metrics are estimated for different time intervals of the peak periods. This section addresses the effect of the choice of the time of day analysis interval (segmentation of the peak period into subintervals) on the calculated metrics.

The available data allows estimating travel time for every minute of the day. However, it may not be useful to produce the reliability metrics for every minute. At the same time, as can be concluded from the earlier discussion, it may not be appropriate to compute the metrics to represent long periods of time. Existing analysis and modeling approaches utilize 15-30 minute intervals suggesting that segmenting the peak period into 15-30 minute intervals may be needed for estimating the reliability metrics for such applications.

In this study, the $80^{\text {th }}$ Percentile PTI metric was computed for analysis periods of 15 minutes, 15 minutes, 1 hour, 2 hour, and 4 hour and the results are presented in Figure 4.16. The results show that depending on the congestion levels, different aggregation periods may lead to significantly different assessments of the reliability. One effect of using more aggregated periods of time would be the dilution of the travel time variability during the period of interest, particularly during periods of varying congested levels. Another potentially undesirable effect of such aggregation is the dilution of the relative differences between the facilities being compared such as the comparison of the GP and HOT lanes, conducted in this study. 


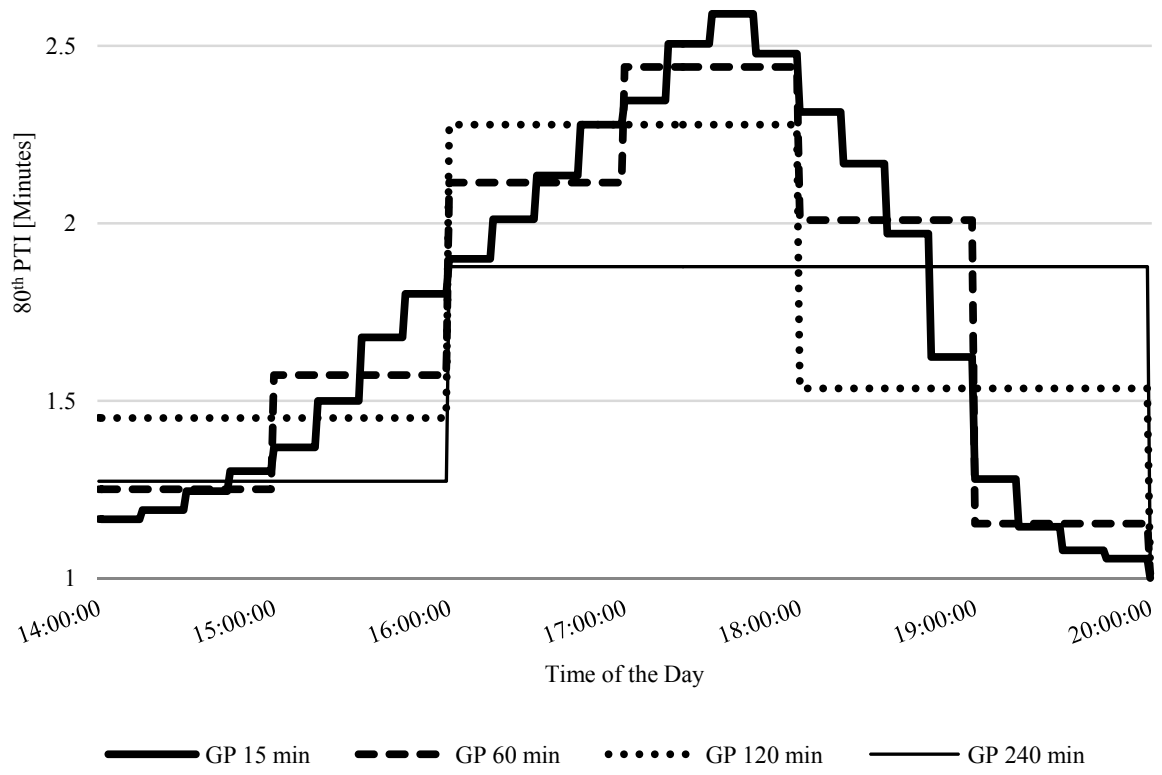

Figure 4.16: Impact of Aggregation Levels on the $80^{\text {th }}$ Percentile PTI Metric

\subsubsection{Length of Data Collection Period}

Another aspect of the travel time reliability researched is the length of the period for which the data is collected as it impacts the accuracy of the computed reliability metrics. This accuracy can be assessed compared to the accuracy achieved if the data is collected for a long period of time (a whole year in this study).

The analysis investigated the root mean square error (RMSE) between the values of the calculated reliability metrics based on data collected for each data collection period length and the metrics values calculated using a year's worth of data. The considered measures are the $95^{\text {th }}$ percentile and $80^{\text {th }}$ percentile PTI for GP and HOT lanes. Figure 4.17 shows how the RMSE of the computed values of reliability measures varies with the number of weeks considered for reliability estimation. The figure shows that as the data collection period increases, the error relative to the estimates based on one 
year's data decreases. However, Figure 4.17 shows that even when collecting data for 40 weeks, the RMSE is still at least $10 \%$ and as high as $18 \%$ for the investigated measures. The SHRP2 LO3 project recommended using at least 6 months and preferably a year's worth of data to estimate the reliability metrics. The results in Figure 4.17 confirm that at least one year's worth of data is needed to estimate stable values of the investigated reliability measures.

Based on the results of the study, at least one year of data is recommended for use to obtain stable values of reliability metrics.

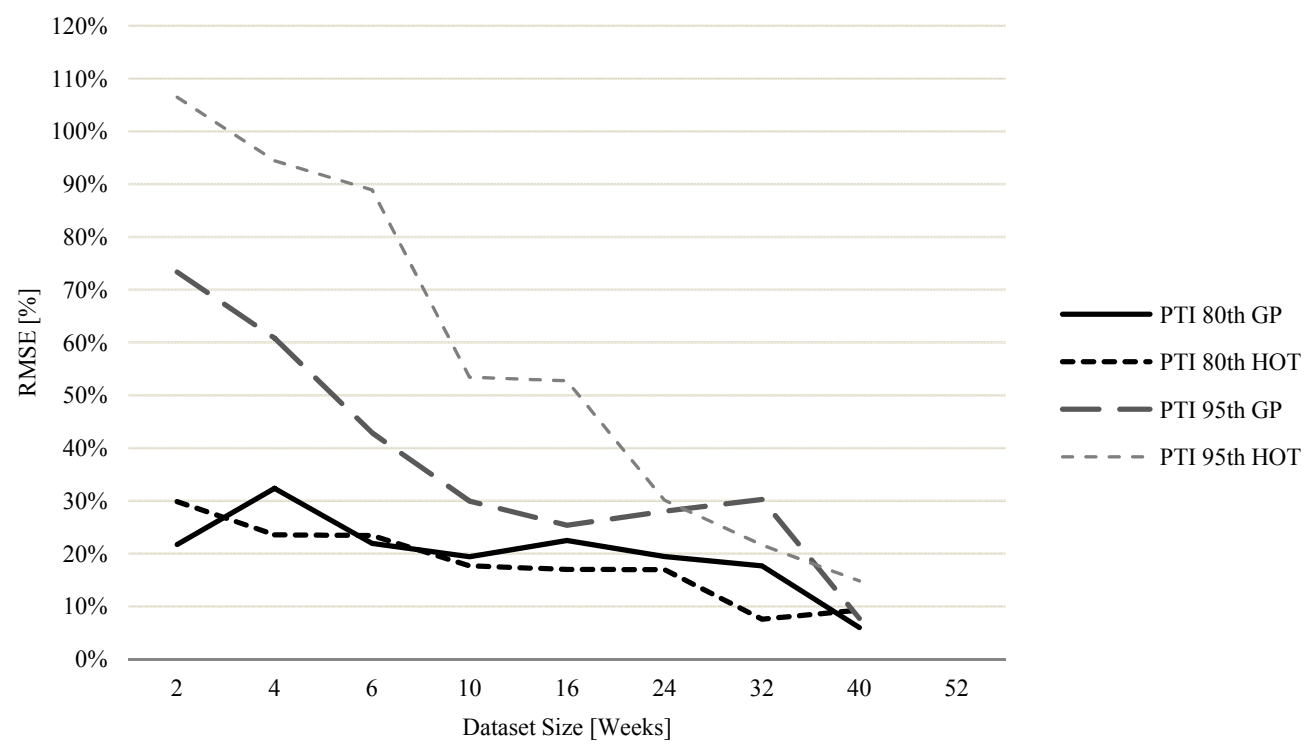

Figure 4.17: Effect of Data Collection Period Length

\subsubsection{Travel Time Reliability Metrics Summary}

There are several metrics of reliability that have been recommended for use. These metrics need to be calculated at relatively fine levels of aggregation of travel time data when used for advanced management strategies and analysis methods. The results show that the parameters of travel time distributions vary during the peak period reflecting the effects of the traffic congestion, traffic flow dynamics, and the proportion of the 
contribution of non-recurrent factors such as incidents to the unreliability of travel time on the investigated facility. Different trends of travel time variations are observed when using different reliability metrics to assess reliability as the congestion level changes during the peak period.

The results also show that examining combinations of time-variant static distribution parameters and reliability metrics of the GP and HOT lanes can provide valuable information that cannot be obtained when performing the analysis at higher aggregation levels. The $95^{\text {th }}$ Percentile PTI, $80^{\text {th }}$ Percentile PTI, and Misery Index showed continuity and sensitivity in their variations in response to the increase in variability as the congestion in the peak hour. The BI measure was insensitive to the increase in congestion on the GP lane but showed sensitivity to the congestion for the HOT lanes due to the difference in the rates of change of standard deviations and medians of travel time with the increase of congestion on these facilities, indicating that the interpretation of the results based on this metric should be done with caution. The FOT and Skew Statistics showed inconsistent patterns of travel time variation with the increase in congestion on GP and HOT lanes reflecting the differences in the rates of the increase in the median and standard deviation rates of travel time with the increased congestion on the two facilities and the relative contributions of non-recurrent events to the unreliability.

The results also show that examining combinations of time-variant static distribution parameters and reliability metrics of the GP and HOT lanes can provide information about the variations of the reliability of these facilities during the analysis periods and the contribution of nonrecurring events at different times of the day. 
The results from the study also confirmed that the use of at least a 30 minute period of analysis is preferable to using longer periods for applications that require fine-grained analysis in order to reasonably represent the reliability pattern during congested periods. In addition, the results from the study confirmed that at least one year's worth of data should be collected to obtain a more stable value of reliability metrics.

\subsection{Adaptive Limited Memory Filter Performance}

The accuracy and robustness of the ALMF is assessed based on a case study that has similar characteristics in terms of performance measures to those found in a priced corridor. The accuracy of the ALMF is assessed by comparing the results from the ALMF algorithm with a model where all parameters are known. On the other hand robustness is assessed by exploring the range of the initial conditions under which the ALMF can produce consistent estimates.

\subsubsection{Case Study}

The case study consists of the estimation of the parameters of a linear model where the measurements have been corrupted by known noise levels $v_{k}$. The linear model $z_{k}$ is defined by a constant $\beta_{0}$ and a time varying component $\beta_{1}$, similar to a utility function with one constant and one observable attribute $x_{k}$. The model specification is shown in Equation 4.2

$$
z_{k}=\left(\begin{array}{ll}
1 & x_{k}
\end{array}\right)\left(\begin{array}{l}
\beta_{0} \\
\beta_{1}
\end{array}\right)+v_{k}
$$


In the experiment, the parameters $\beta_{0}$ and $\beta_{1}$ are kept fixed while the signal $z_{k}$ is corrupted by a normally distributed noise with zero mean and known standard deviation. The noise variance levels and the sampling frequency in the case study are chosen accordingly to the typical values observed for the daily time series of the proportion of the HOT users in I-95. As mentioned in previous sections, the standard deviation of the proportion of HOT users varies during the day in a range between 0.3 and 0.1 (the higher value correspond to low demand periods and vice versa); thus, we consider these values as the basis for the noise added. For the sampling frequency (readings per time unit, length of the measurement time series), considering 5 minutes aggregation level in the data, results in having 288 measurements in total per day. Thus, the measurement series used in this exercise will be consistent with this number.

Three study cases are considered; two of them focus on the effect of different noise levels on the parameter estimation quality and one case focuses in the effect of time varying noise on the parameter estimation quality. The details of each case are as follows:

- Case I: A normally distributed noise $v_{k} \sim N(0,0.1)$ applies to all measurements in the time series

- Case II: A normally distributed noise $v_{k} \sim N(0,0.3)$ applies to all measurements in the time series

- Case III: A normally distributed noise $v_{k} \sim N(0,0.1)$ applies to half of the measurements while $v_{k} \sim N(0,0.3)$ applies to the remaining half of the measurements. 
The value of the parameters $\beta_{0}$ and $\beta_{1}$ in the model, are arbitrarily kept as -1 . A summary of the used parameters for the different cases in study is given in Table 4.3

Table 4.3: Parameter Values Used in ALMF Testing

\begin{tabular}{|c|c|c|c|}
\hline \multirow{2}{*}{ CASE } & \multicolumn{2}{|c|}{$z_{k}=\beta_{0}+\beta_{1} x_{k}$} & $v_{k} \sim N(0, \sigma)$ \\
\cline { 2 - 3 } & $\beta_{0}$ & $\beta_{1}$ & $v_{k} \sim N(0,0.1) \forall k \in[1, \ldots, 288]$ \\
\hline I & -1 & -1 & $v_{k} \sim N(0,0.3) \forall k \in[1, \ldots, 288]$ \\
\hline II & -1 & -1 & $v_{k} \sim N(0,0.1) \forall k \in[1, \ldots, 144] v_{k} \sim N(0,0.3) \forall k \in[145, \ldots, 288]$ \\
\hline III & -1 & -1 & \\
\hline
\end{tabular}

Similarly, the variation pattern of the independent variable (observable attribute) is chosen such that the variables closely resemble the daily variation of traffic performance measures; such as, travel time, reliability, or fare in the section of interest in I-95. Figure 4.18 shows the daily variation pattern of some typical performance measures observed on the HOT lanes. Figure 4.19 shows the assumed variation pattern of the independent variable in the experiment.

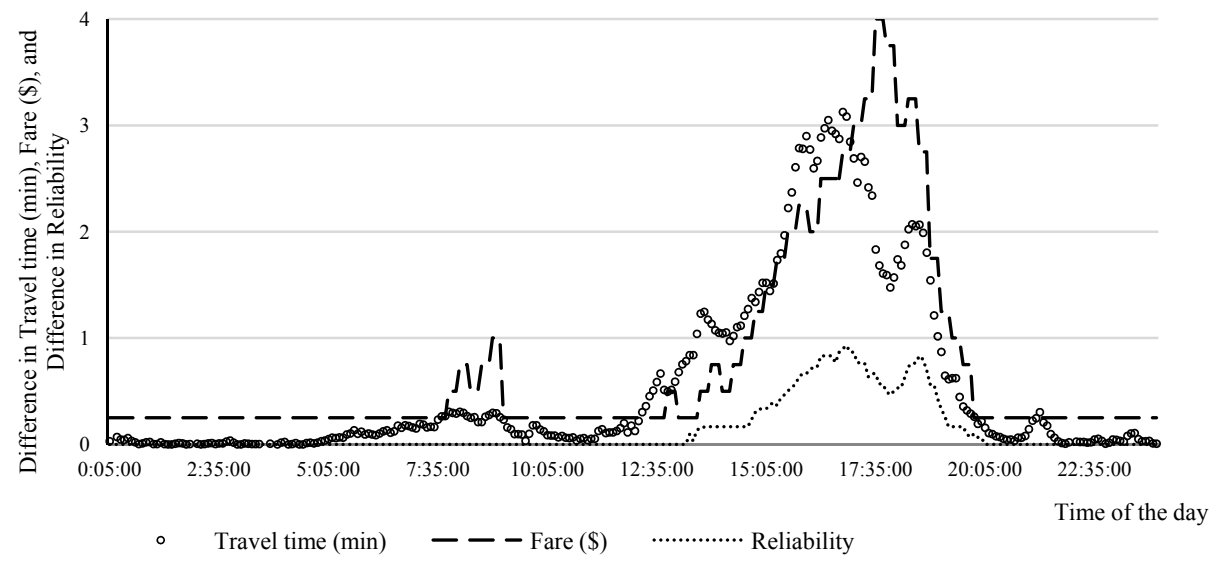

Figure 4.18: Typical Daily Performance Measures Variation in I-95 


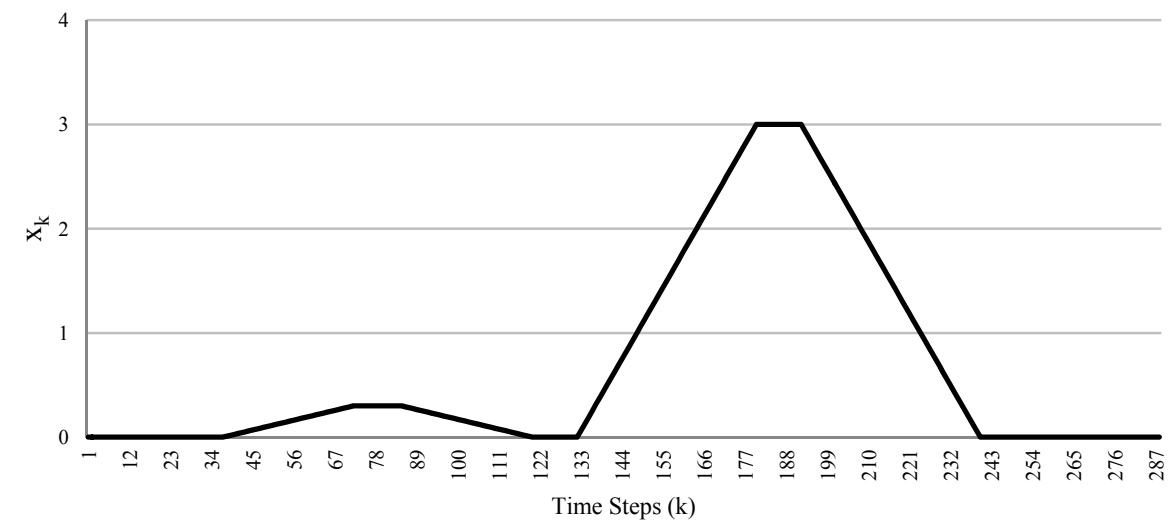

Figure 4.19: Pattern of Variation of $x_{k}$ as Used in the Experiment

Once the measurement data is computed according to the values of $\beta_{0}$ and $\beta_{1}$, a simulated noise signal is generated and added to the measurement time series. Figures 4.20 to 4.22 show the resulting measurement time series for each case study.

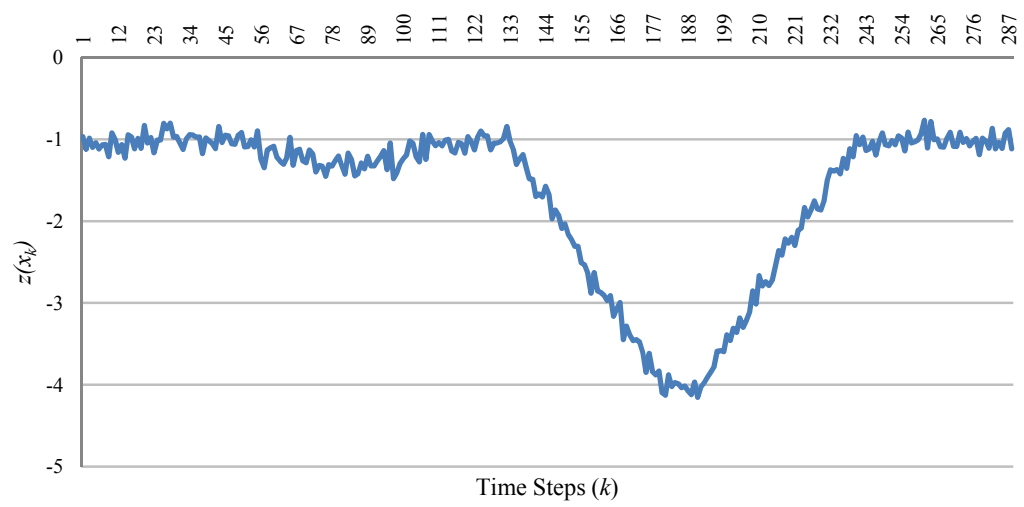

Figure 4.20: Measurement Time Series Case I 


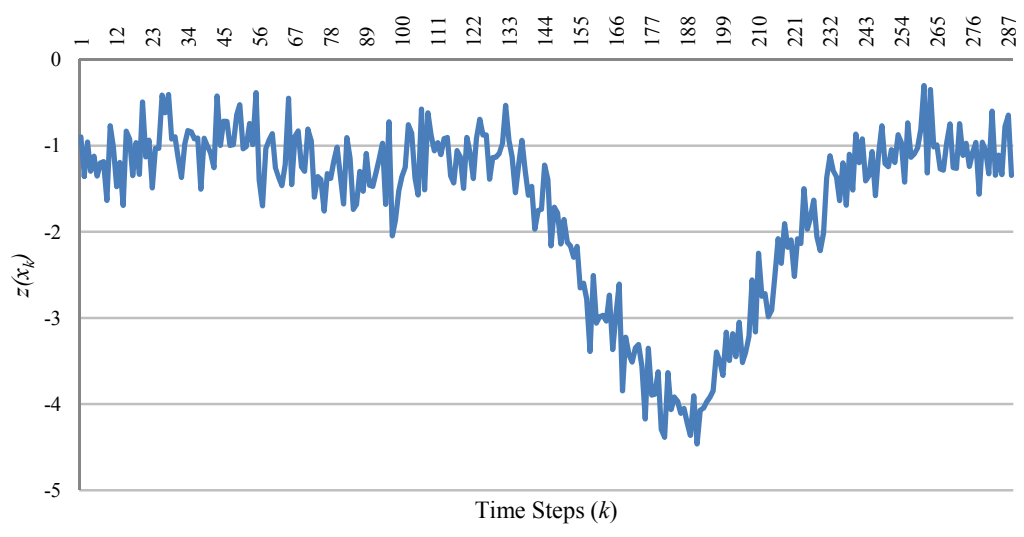

Figure 4.21: Measurement Time Series Case II

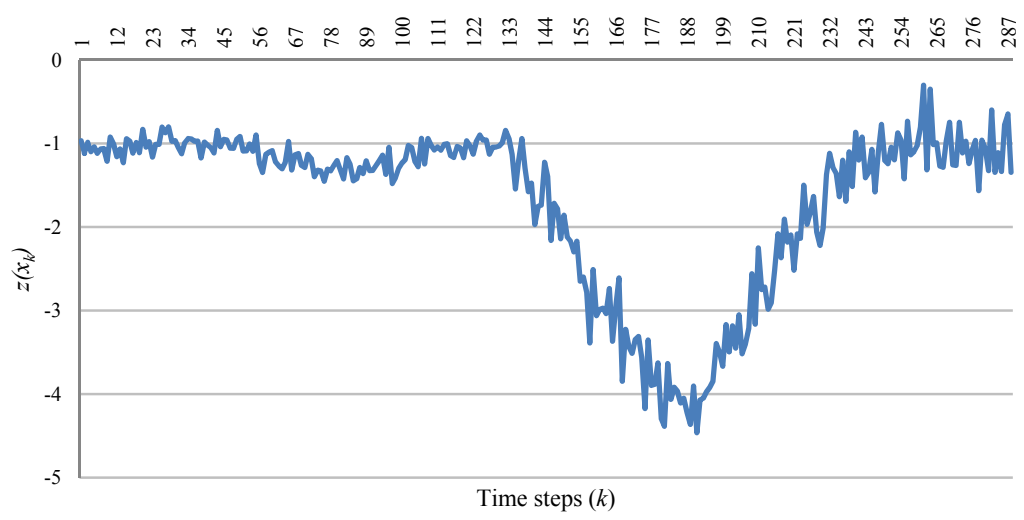

Figure 4.22: Measurement Time Series Case III

\subsubsection{Analysis of ALMF Performance}

Testing the performance of the ALMF algorithm requires the a priori specification of a number of initial values and parameters. Such values need to be adjusted in order to ensure that the obtained parameters are optimal in the sense of providing minimum variance estimates when applying the AMLF algorithm. In this initial stage, arbitrary initial values (although nor far from optimal) are used to obtain insight about the ALMF algorithm performance for the study cases. However, the robustness of the ALMF algorithm in regard to changes on the set of initial values is explored later in this section. 
The set of initial values used in this investigation is given in Table 4.4.

Table 4.4: Parameter values used in ALMF testing

\begin{tabular}{|l|c|l|c|}
\hline Parameter & Value & Parameter & Value \\
\hline$\beta_{0}$ & -1.0 & $q_{2}$ & 0.0 \\
\hline$\beta_{1}$ & -1.0 & $Q_{11}$ & 0.1 \\
\hline$P_{11}$ & 0.1 & $Q_{12}$ & 0.0 \\
\hline$P_{12}$ & 0.0 & $Q_{21}$ & 0.0 \\
\hline$P_{21}$ & 0.0 & $Q_{22}$ & 0.1 \\
\hline$P_{22}$ & 0.1 & $r$ & 0.0 \\
\hline$q_{1}$ & 0.0 & $R$ & 0.1 \\
\hline
\end{tabular}

Considering this, for each case study, different windows sizes ranging from 3 to 12 time steps (15 to 1 hour if we consider that each time step accounts for 5 minutes) were used to estimate the model parameters $\hat{\beta}_{0}, \hat{\beta}_{1}$. Later these parameters are fed back to produce an estimated measurement signal $\hat{z}_{k}(\hat{\beta})$ that can be compared to the original measurement $\operatorname{signal} z_{k}$. As a measure of model fit, the mean residual sum of squares $(\mathrm{M} R S S)$ was used to judge the error between the original and estimated measurement series. The MRSS is defined as follows:

$$
M R S S=\frac{1}{N} \sum_{k=1}^{N}\left[z_{k}-\hat{z}_{k}(\hat{\beta})\right]^{2}
$$

Figure 4.23 shows the MRSS values obtained after using the ALMF algorithm and the conventional KF algorithm with $Q$ and $R$ fixed through all the estimation process. From the figure, several observations are possible:

1. As expected, the higher the signal to noise ratio (SNR: ratio between the variance of the signal and the variance of the noise) the higher the RSS. The estimated SNR in the second period is 0.73 and 73.0 for period 6 in Case I 
whilst for Case II the figures are 0.08 and 8.16 for the second and sixth periods respectively (Case III is simply 0.73 for period 2 and 8.16 for period 6). This is shown in the chart where the MRSS is higher for all the cases with higher noise values for all the evaluated periods. In other words, as the information is hidden by higher levels of noise, the accuracy of the estimates is reduced and consequently the accuracy of the reproduced measurement signal is also reduced.

2. When considering the results for Case III, we notice that the MRSS matches the RSS values observed in Case I for periods 2 to 5 and then matches the MRSS values observed in Case II for periods 6 to 9 . In other words, the ALMF algorithm is able to adjust its parameters according to the noise changes on the measurement signal, and in this way provide the best estimation possible, regardless of the changes in the noise statistics.

3. Finally, the results obtained from applying the KF filter to Case I show that the AMLF algorithm can always provide a better measurement series estimation likely due to the lack of flexibility of the KF to adaptively adjust the values of the time varying values of $Q$ and $R$.

In any case, for the levels of noise considered in this study, both the ALMF and the $\mathrm{KF}$ are able to provide a good approximation of the original measurement series as shown in Figure 4.24 


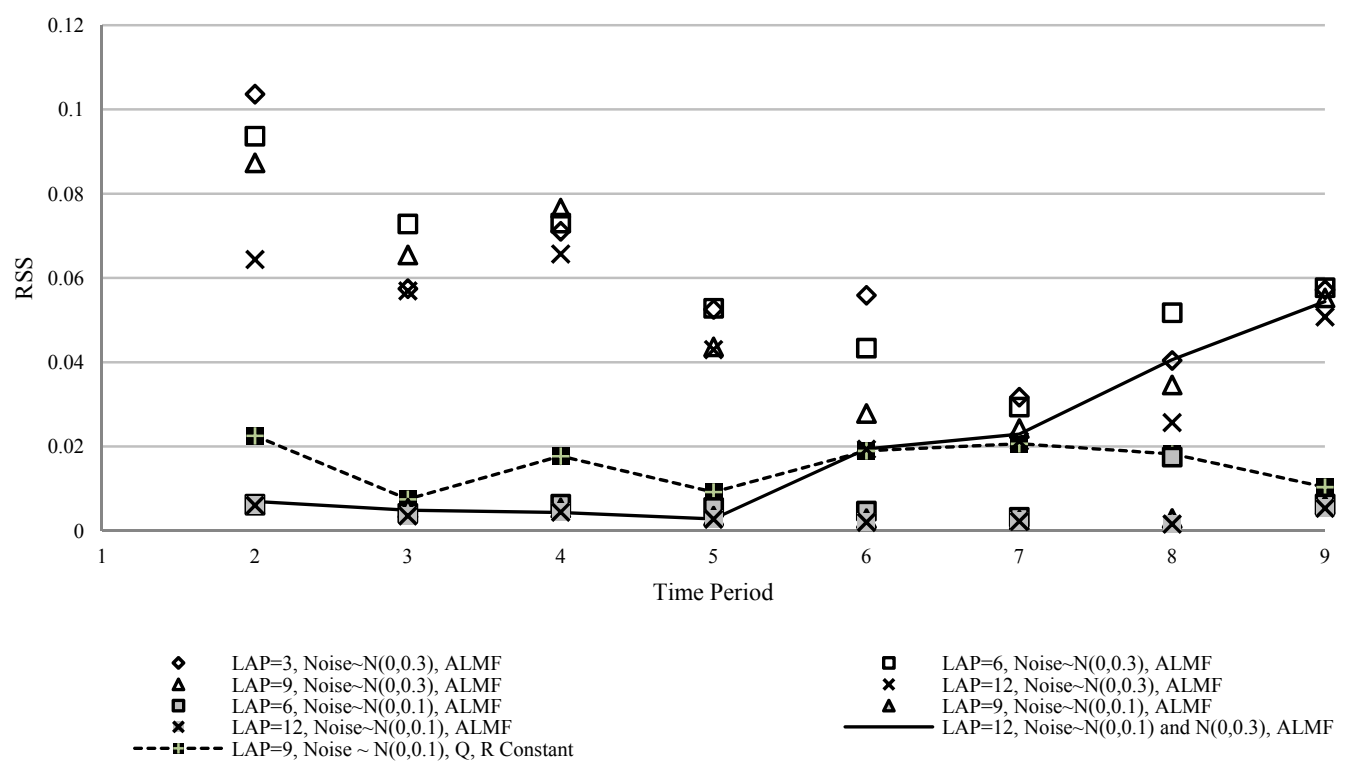

Figure 4.23: RSS Between Observed Measurement $z_{k}$ and Estimated Measurement $\hat{z}_{k}(\hat{\beta})$

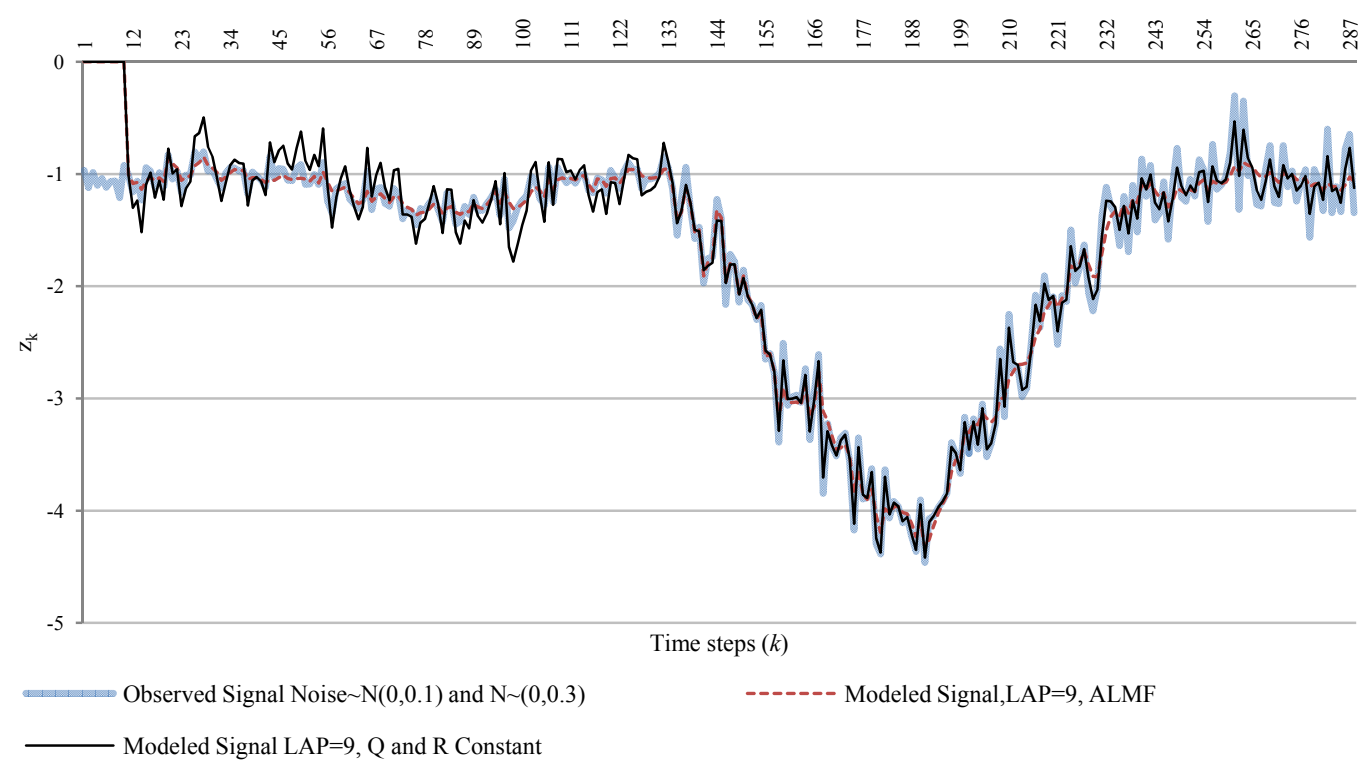

Figure 4.24: Observed and modeled measurement signal using ALMF and KF 


\subsubsection{Optimal LAP Selection}

Because the ALMF algorithm is designed to compensate adaptively for time-varying noise statistics, the natural initial question is how to decide the memory size window (LAP) from where the state and observation samples will be taken. The question is investigated considering that the optimal window size is such that it produces $\hat{\beta}_{0}, \hat{\beta}_{1}$ values closer to the actual parameters while at the same time their covariance is minimized. Figure 4.25 shows the mean relative percent error $(M R P E)$ between the actual $\beta_{0}, \beta_{1}$ and the estimated $\hat{\beta}_{0}, \hat{\beta}_{1}$ for various LAP values in Case I. MRPE is defined as follows:

$$
M R P E_{i}=\frac{1}{288 \beta_{i}} \sum_{j=1}^{288}\left|\beta_{i}-\hat{\beta}_{i}^{j}\right|, i \in\{0,1\}
$$

From Figure 4.25 is possible to infer that there exists an optimal value of the LAP such that the $R P E$ between $\beta_{0}, \hat{\beta}_{0}$ and $\beta_{1}, \hat{\beta}_{1}$ is minimized. However the optimal LAP is different depending on the estimate. This is due to the fact that a LAP too short is not enough to capture the measurement variance accurately whereas a LAP too long may include some samples that are not representative for the time step at which the ALMF is performing the estimation. 


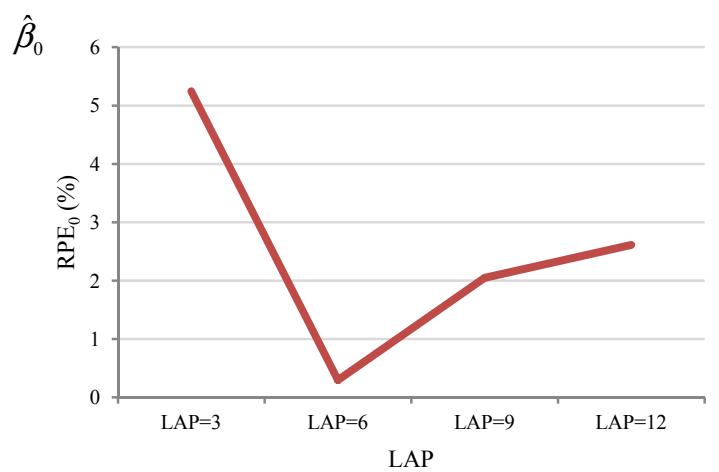

(a) $\beta_{0}$

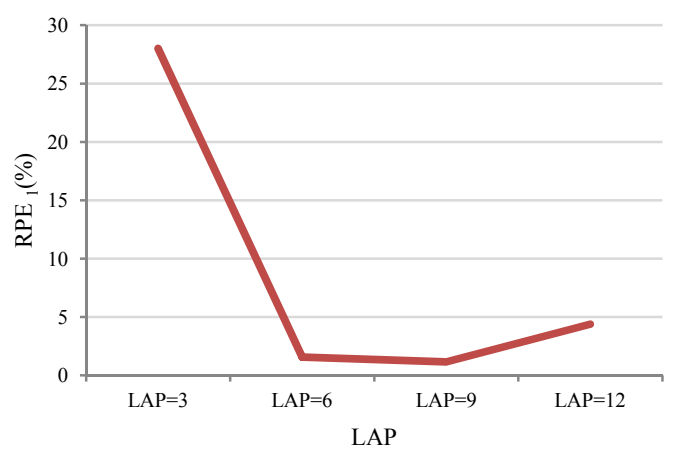

(b) $\beta_{1}$

Figure 4.25: RPE for $\hat{\beta}_{0}$ and $\hat{\beta}_{1}$ Respectively Case I

Conversely, Figure 4.26 and Figure 4.27 show $\hat{\beta}_{0}$ and $\hat{\beta}_{1}$ covariance as a function of time for Case I and Case III respectively. In the figures, ALMF and KF filtering approaches are included. In terms of the time variation of the estimates covariance, it is observed that there are significant differences depending on the value of the LAP used. In both cases, using a value of $\mathrm{LAP}=6$ together with the ALMF algorithm, ensures the optimality of the estimates for any time of the day while the use of the standard KF provides significantly higher variance estimates throughout the day.

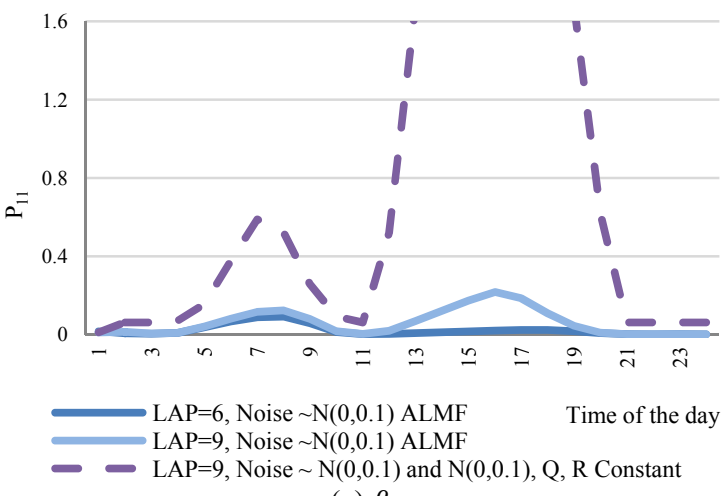

(a) $\beta_{0}$

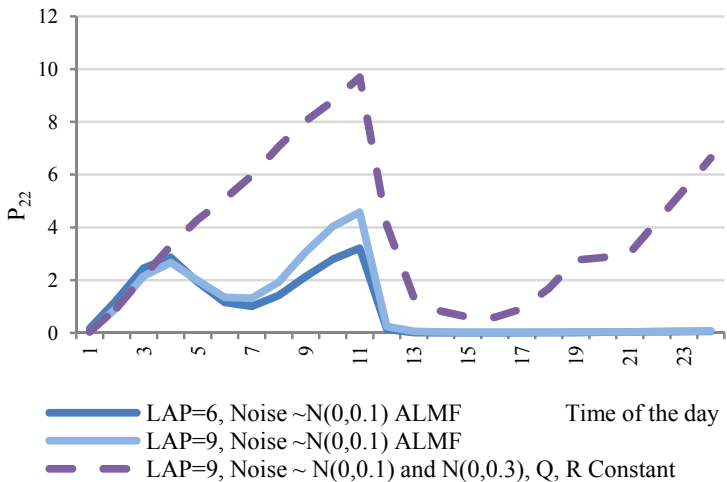

(b) $\beta_{1}$

Figure 4.26: Case I variance for $\hat{\beta}_{0}$ and $\hat{\beta}_{1}$ Respectively 


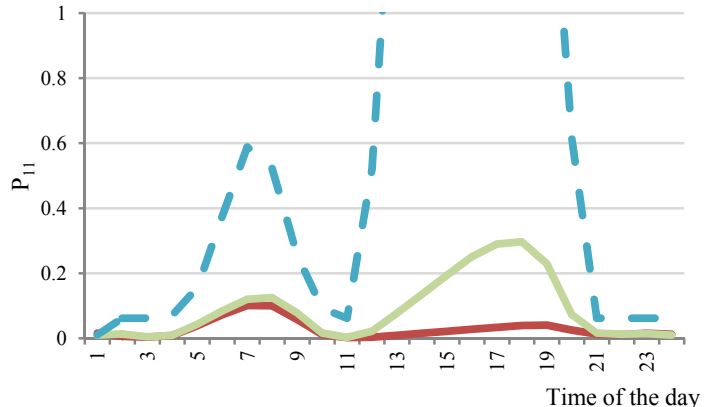

LAP $=6$, Noise $\sim \mathrm{N}(0,0.1)$ and $\mathrm{N}(0,0.3)$ ALMF

LAP $=9$, Noise $\sim \mathrm{N}(0,0.1)$ and $\mathrm{N}(0,0.3)$ ALMF

$\longrightarrow$ LAP $=9$, Noise $\sim \mathrm{N}(0,0.1)$ and $\mathrm{N}(0,0.3), \mathrm{Q}, \mathrm{R}$ Constant

(a) $\beta_{0}$

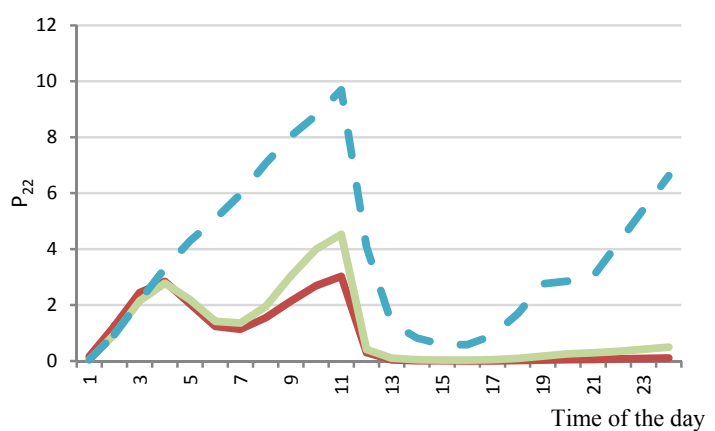

LAP $=6$, Noise $\sim \mathrm{N}(0,0.1)$ and $\mathrm{N}(0,0.3)$ ALMF

LAP $=9$, Noise $\sim \mathrm{N}(0,0.1)$ and $\mathrm{N}(0,0.3)$ ALMF

- LAP $=9$, Noise $\sim \mathrm{N}(0,0.1)$ and $\mathrm{N}(0,0.3), \mathrm{Q}, \mathrm{R}$ Constant

(b) $\beta_{1}$

Figure 4.27: Case III variance for $\hat{\beta}_{0}$ and $\hat{\beta}_{1}$ Respectively

Finally, Figure 4.28 and Figure 4.29 show the values of $\hat{\beta}_{0}, \hat{\beta}_{1}$ as a function of time and filtering approach used for Case I. The figure highlights the superior performance of the ALMF algorithm by means of systematically providing more accurate estimates than the KF for any time of the day if the proper LAP value is used.

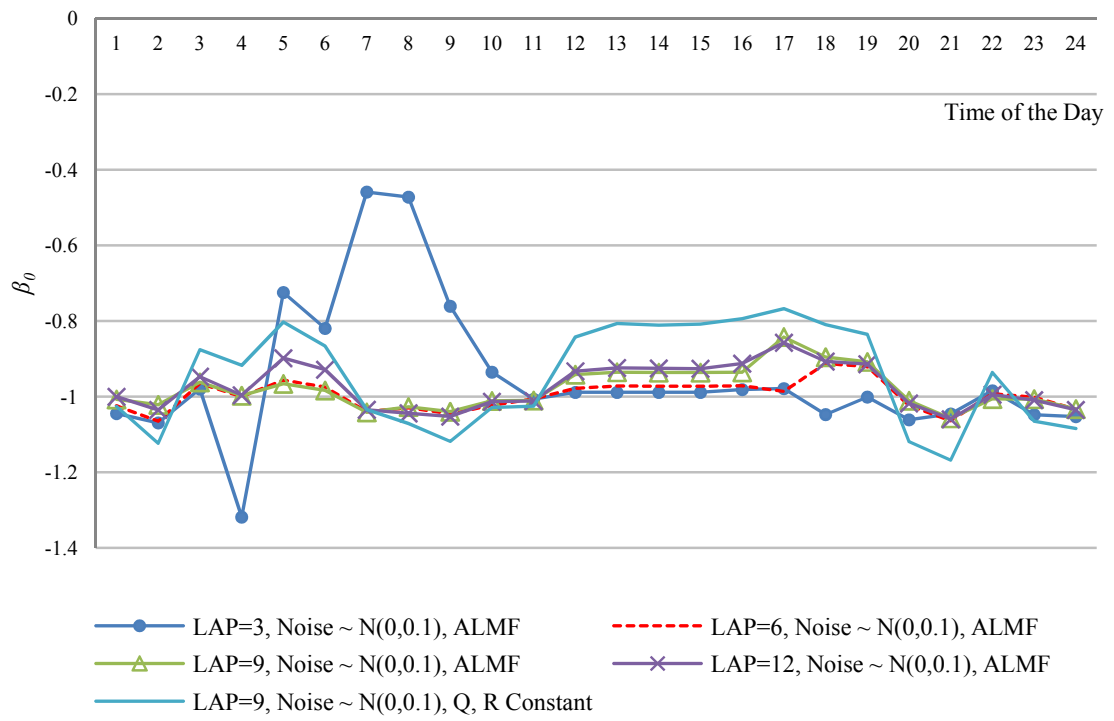

Figure 4.28: Hourly $\hat{\beta}_{0}$ estimates 


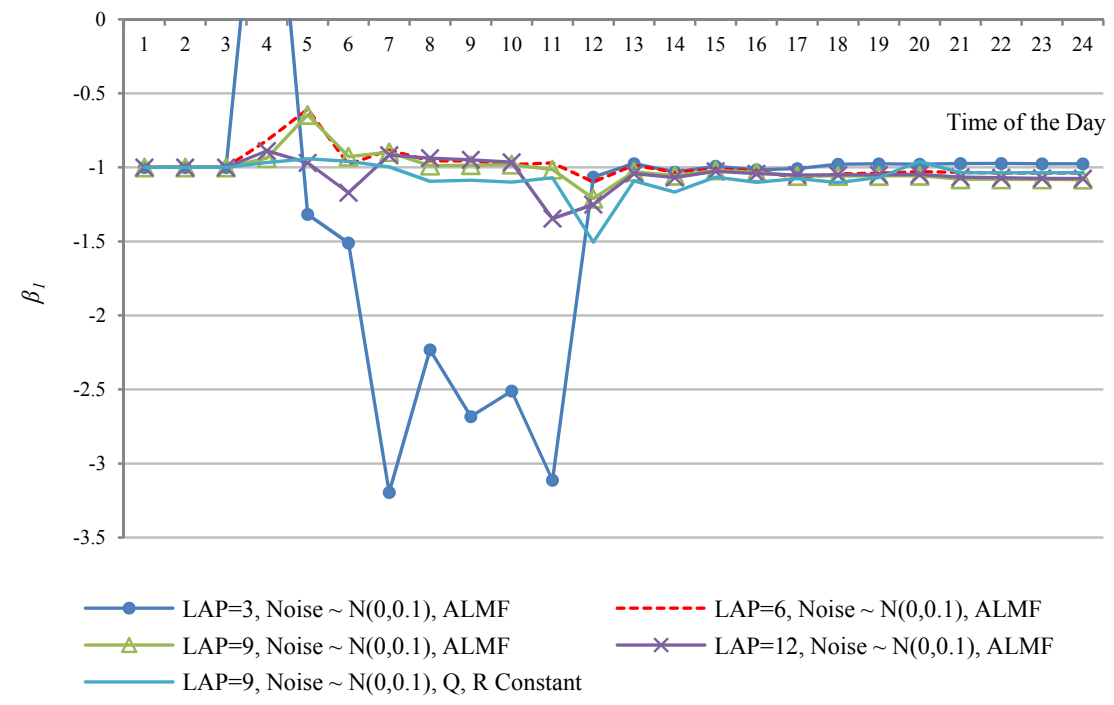

Figure 4.29: Hourly $\hat{\beta}_{1}$ estimates

\subsubsection{Robustness of the ALMF}

In the previous section, the performance of the ALMF algorithm was tested assuming a set of default parameters. In this section the robustness of the ALMF in regard to variations in those parameters is tested. For this purpose, Case I using LAP $=6$ and the ALMF is considered. The robustness of the algorithm is tested by changing one parameter at the time while leaving the rest of them ceteris paribus. Table 4.5 shows the range of variation considered for each initial parameter value. 


\section{Table 4.5: Parameter values used in ALMF testing}

\begin{tabular}{|l|c|}
\hline Parameter & $\begin{array}{c}\text { Range of } \\
\text { variation }\end{array}$ \\
\hline$\beta_{0}$ & {$[0.0,5.0]$} \\
\hline$\beta_{1}$ & {$[0.0,5.0]$} \\
\hline$P_{11}$ & {$[0.0,1.0]$} \\
\hline$P_{22}$ & {$[0.0,1.0]$} \\
\hline$q_{1}$ & {$[-1.0,5.0]$} \\
\hline$q_{2}$ & {$[-1.0,5.0]$} \\
\hline$Q_{11}$ & {$[0.0,5.0]$} \\
\hline$Q_{22}$ & {$[0.0,5.0]$} \\
\hline$r$ & {$[-0.3,0.3]$} \\
\hline$R$ & {$[0.1,0.5]$} \\
\hline
\end{tabular}

The results of the sensitivity analysis are provided in Figures 4.30 to Figure 4.35 and from them several general observations are possible:

1. For the case study, the estimates $\hat{\beta}_{0}$ and $\hat{\beta}_{1}$ are sensitive to the initial values of the parameters and a tuning process seems to be advisable prior to the implementation of the ALMF. In this case, where all the actual parameters of the signal are known, it is relatively easy to define a range where testing the response of the ALMF algorithm, however in practical applications where a priori information of the parameters is unknown In such case, the range of exploration must be defined by means of an exploratory effort in order to ensure the optimality of the obtained parameters.

2. The initial values used in the estimation are particularly important in those processes where the relationship between the noise and the signal variances is low. That is to say, providing better initial values may help the ALMF algorithm to stay close the true estimates even though the information 
contained in the original signal is poor and not enough to perform robust estimation. This can be observed in all the figures in the period of time referred as "low SNR period" where relatively small deviations from the true values of the parameters can lead to significant changes in the value of the estimates. On the other hand, it is observed that when the signal variance is higher than the noise variance, the so called "high SNR period," the relevance of the initial values used is negligible due to the fact that in general, regardless the choice of these values, the ALMF algorithm can always manage to perform robust estimation by using the information contained on the measurement signal. This is particularly true for the time-varying component of the measurement signal.
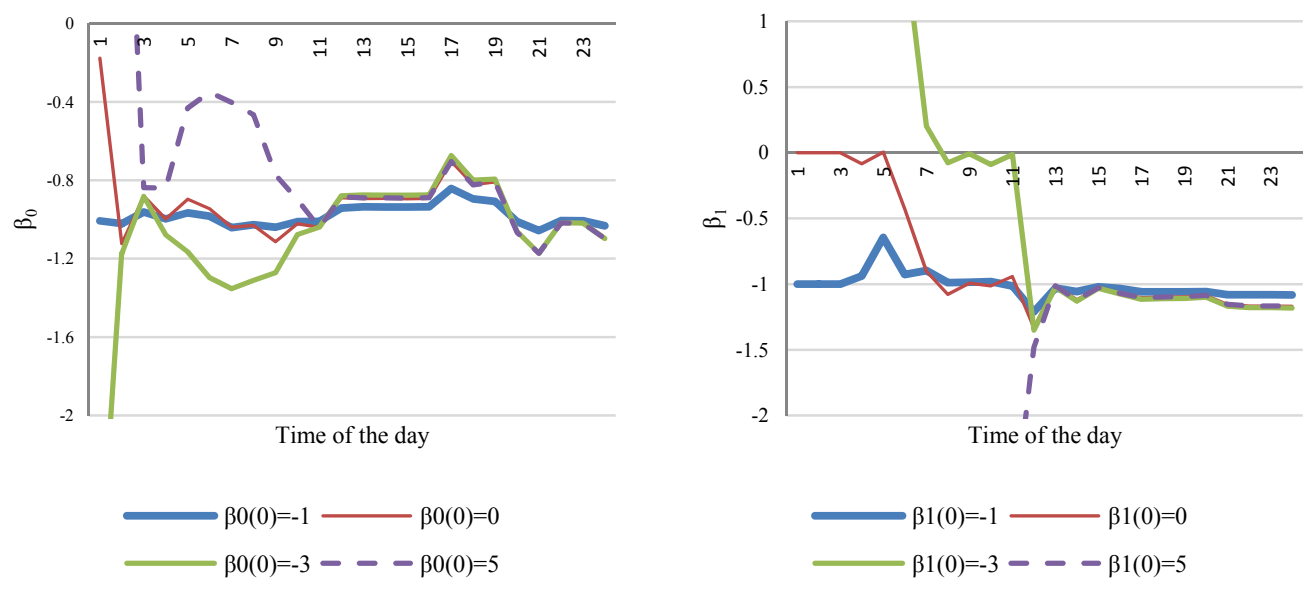

Figure 4.30: ALMF algorithm robustness to $\hat{\beta}_{0}$ and $\hat{\beta}_{1}$ initial values 

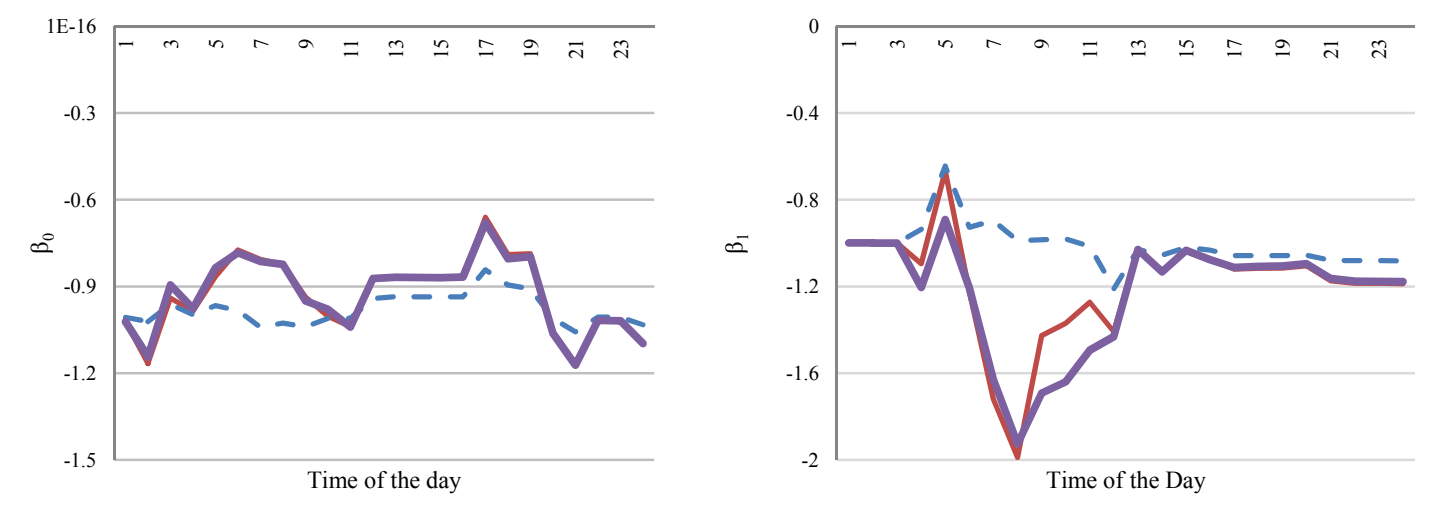

$\longrightarrow \mathrm{P} 11(0)=0.0-\longrightarrow \mathrm{P} 11(0)=0.1 \longrightarrow \mathrm{P} 11(0)=0.3$

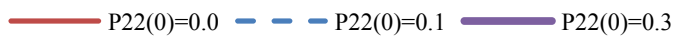

Figure 4.31: Hourly $\hat{\beta}_{0}$ and $\hat{\beta}_{1}$ for different initial values of $\mathbf{P}_{11}$ and $\mathbf{P}_{22}$
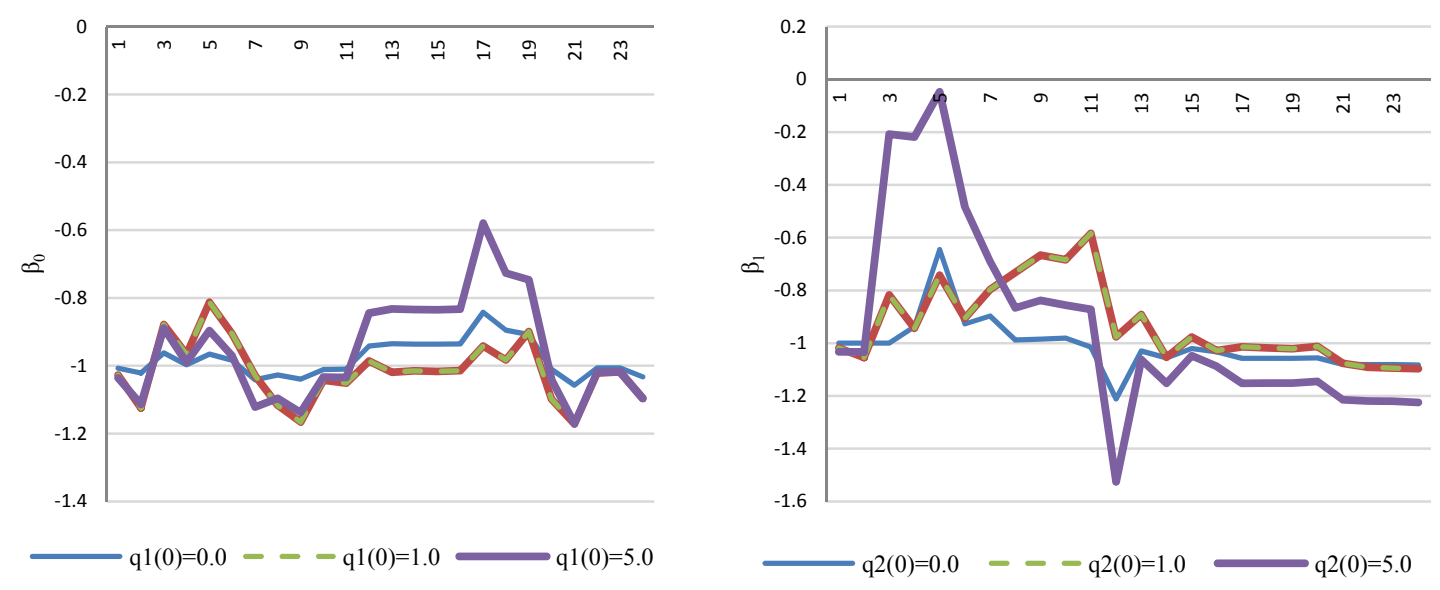

Figure 4.32: Hourly $\hat{\beta}_{0}$ and $\hat{\beta}_{1}$ for different initial values of $q_{1}$ and $q_{2}$
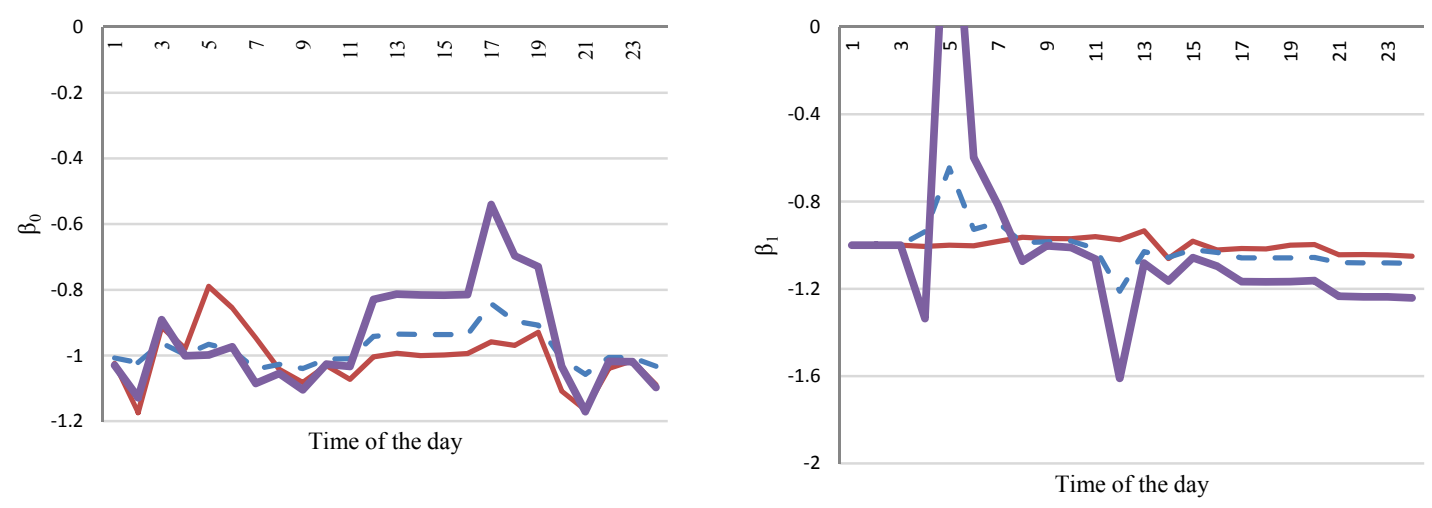

$\longrightarrow \mathrm{Q} 11(0)=0.0--\mathrm{Q} 11(0)=0.1 \longrightarrow \mathrm{Q} 11(0)=5.0$

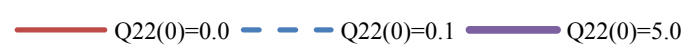

Figure 4.33: Hourly $\hat{\beta}_{0}$ and $\hat{\beta}_{1}$ for different initial values of $Q_{11}$ and $Q_{22}$ 

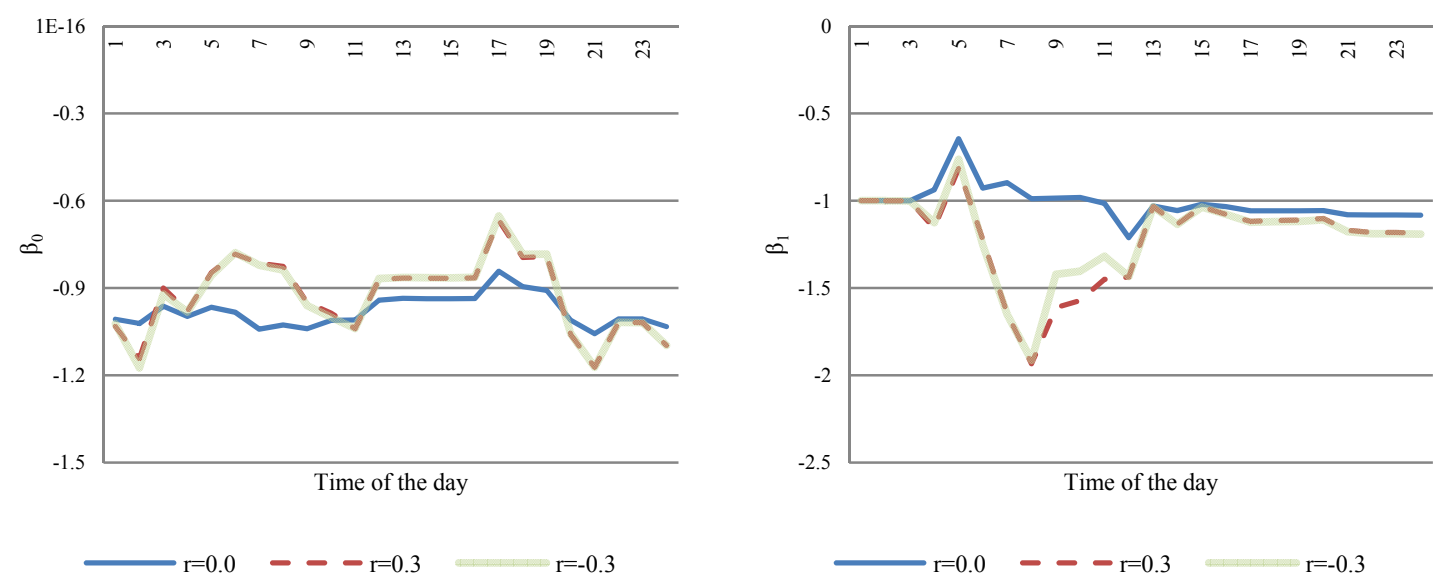

Figure 4.34: Hourly $\hat{\beta}_{0}$ and $\hat{\beta}_{1}$ for different initial values of $r$
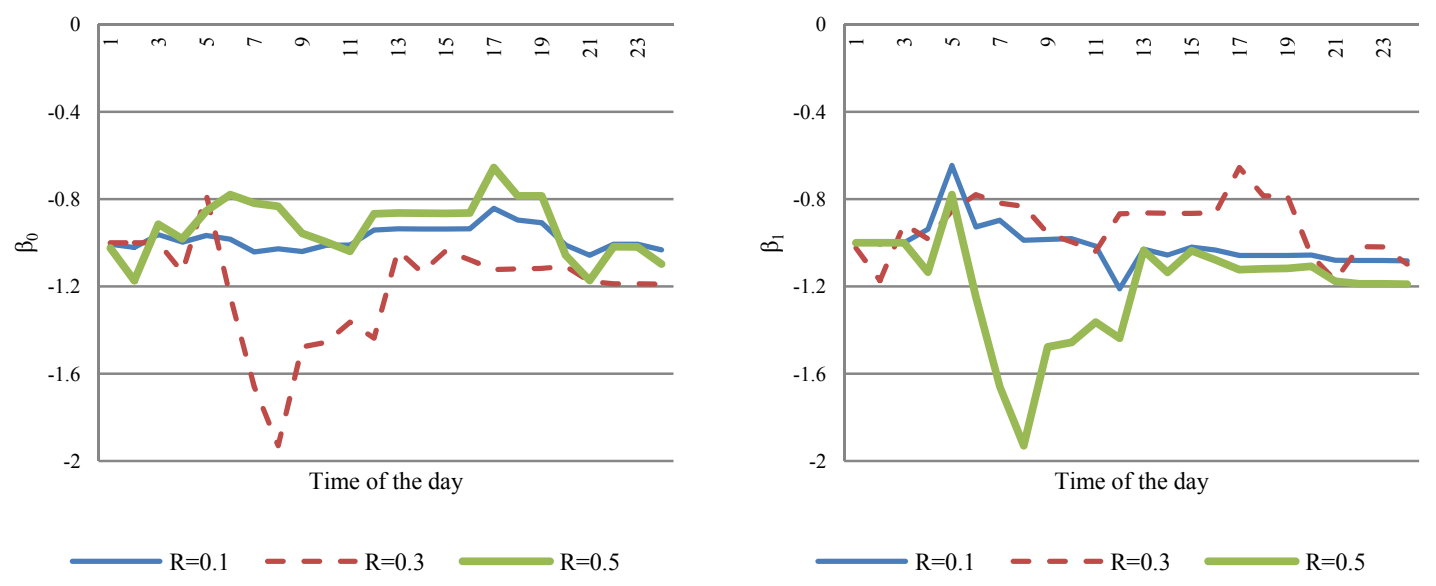

Figure 4.35: Hourly $\hat{\beta}_{0}$ and $\hat{\beta}_{1}$ for different initial values of $\boldsymbol{R}$ 


\section{CHAPTER 5}

\section{SENSITIVITIES TRAVEL TIME AND TRAVEL TIME RELIABILITY IN A ROAD PRICING ENVIRONMENT}

In this chapter, the time of the day proportion of the HOT users by time of day on a freeway in South Florida is explained as a function of the observed attributes of the system; namely, travel time, travel time reliability, and toll. The choice between the HOT or the GP lanes is considered as a binary choice and it is modeled using the well know logit model where the utility functions explicitly consider the differences in the attributes of each alternative. Time of the day parameter estimation of the logit model is performed using the ALMF algorithm developed in Chapter 4. Finally, the time varying results are combined and parameter distributions are built for each time of the day, considering day-to-day variations. The significance of the explanatory variables is assessed by constructing time varying confidence intervals for each estimate.

\subsection{Estimation Framework}

As mentioned in Chapter 2, there is a lack of empirical data to estimate the necessary time varying parameters to assess the behavior of the users in a road pricing setup. That comes from the fact that traditionally, user behavior as related to the estimation of the user sensitiveness to travel time or travel time reliability has been mostly approached using individual data collected by means of either stated preferences surveys (SP), reveled preferences surveys (RP), or a mix of the two. Notwithstanding the advantages of such surveys; such as, allowing the capture of accurate motorist socio-demographic characteristics, travel patterns, and travel purposes; the required efforts and costs prohibit 
conducting surveys on a regular basis. In addition, data collected using such methods are normally representative of average conditions or a few days that may not capture temporal variations in user values and other trip features, including user behavior under non- recurrent congestion conditions (incidents, weather, etc.).

In this study, the base for the estimation of user sensitivities to travel time and travel time reliability, comes from the fact that the impacts of motorist's preferences are continuously revealed and captured by traffic detection devices as they measure changes in transportation system conditions. These responses can be extracted by mining ITS detector data archived by traffic management centers (TMC) operational files and associating these responses with changes in transportation system variables such as travel time, travel time reliability, toll, and so on. The traveler responses and the transportation system variables can be linked together by means of a model with unknown parameters that can be estimated to minimize a measure of the error between observed and modeled motorists' responses.

A convenient setup to evaluate the feasibility of this approach is when HOT lanes with dynamic pricing policy are part of the transportation system. Traffic operation data when combined with travel time, travel time reliability, and toll data allow the investigation of the logic behind the observed aggregate driver's behavior as related to the relative differences between the attributes of the HOT lanes versus the GP lanes. 


\subsection{Model Definition}

As a working hypothesis we state that when deciding whether to use HOT or GP, travelers select the alternative that minimizes some measure of their trip disutility. The aggregated choice made by drivers can be modeled as a binary Logit model where the probability of using the priced facility can be described as a function of the perceived trip disutility. Likewise, the trip disutility can be estimated as a function of the trip attributes for each travel alternative. Such approach is mathematically described in Equation 4.1, in which $P_{k}$ is the proportion of motorists using the HOT facilities during a discrete period of time $k$ and $U_{k}^{i}$ represents the utility of each option as perceived by drivers during the same period of time $k$. For the cases, where the trip options are HOT and GP lanes, $P_{k}$ can be written as:

$$
P_{k}=\frac{e^{U_{k}^{H O T}}}{e^{U_{k}^{G P}}+e^{U_{k}^{H O T}}}
$$

With regard to the utility function, a common approach has been the use of a linear combination of observable trip attributes where the weight on each attribute can be interpreted as the marginal contribution of each attribute to the total trip utility as perceived by the motorist. When the travel time $T T_{k}^{i}$, the travel time reliability $R_{k}{ }^{i}$ and the toll $F_{k}{ }^{i}$ are used to represent travel attributes, the utility function for each alternative is given by Equation 4.2 where $B_{k}{ }^{i}$ is a time dependent constant (bias) and $i=\{H O T, G P\}$.

$$
U_{k}^{i}=B_{k}^{i}+V O T_{k} \times T T_{k}^{i}+V O R_{k} \times R_{k}^{i}-F_{k}^{i}
$$

When the utility function in Equation 5.2 is measured in monetary units, the travel time weight in the utility function $\left(V O T_{k}\right)$ represents directly the willingness to pay per unit of time to increase driver's utility. Similarly, the weight $\left(V O R_{k}\right)$ in the reliability 
component of the utility function represents the willingness to pay per unit of trip reliability to increase the trip utility. Furthermore, the toll does not need any weight because it is already expressed in the same units as the utility function. Finally, a constant value in the utility function is considered in order to capture other factors or biases not explicitly included in the utility function.

Combining Equations (5.2) and (5.1) yields to the linear system shown in equation (5.3) with the unknown parameters $B_{k}, V O T_{k}$, and $V O R_{k}$, measured proportions of HOT users $P_{k}$ and trip attributes $T T_{k}^{i}, R_{k}^{i}$ and $F_{k}^{i}$.

$$
\ln \left(\frac{1}{P_{k}}-1\right)=-B_{k}+V O T_{k} \times\left(T T_{k}^{G P}-T T_{k}^{H O T}\right)+V O R_{k} \times\left(R_{k}^{G P}-R_{k}^{H O T}\right)+F_{k}^{H O T}
$$

The question now is how to estimate the parameters $B_{H O T}(k), V O T(k)$, and $\operatorname{VOR}(k)$ for each time interval $k$ in such a way that a measure of the difference between observed and modeled $P_{k}$ is minimized.

\subsection{The ALMF Algorithm Setup}

The Kalman filter and its adaptive extension ALMF algorithm, as explained in Chapter 4, are a set of mathematical equations that are used in this study to provide an efficient recursive way to estimate the parameters in Equation (5.3) for each time interval $k$ in a way that the variance of the parameter estimates is minimized.

The filter approach uses a system dynamics model (i.e., binary Logit in our case) and a set of time varying measurements (proportion of the HOT users) to form an estimate of the system varying state parameters that are better than the estimates obtained by using any one measurement alone. The filtering approach estimates the model parameters $\left(V O T, V O R\right.$ and $\left.B_{H O T}\right)$ as the weighted average between the parameters 
estimated at the previous time steps and the noisy measurements available at time $k$. This formulation is mathematically described in general as shown in Equation 5.4 and in particularin Equation 5.5for solving the problem stated in Equation 5.3.

$$
\begin{gathered}
\hat{x}_{k}=\hat{x}_{k}^{-}+K_{k}\left(z_{k}-H \hat{x}_{k}^{-}\right) \\
\left\{\begin{array}{c}
-B_{k} \\
V O T_{k} \\
V O R_{k} \\
1
\end{array}\right\}=\left\{\begin{array}{c}
-B_{k}^{-} \\
V O T_{k}^{-} \\
V O R_{k}^{-} \\
1
\end{array}\right]+K_{k}\left(\ln \left(\frac{1}{P_{k}}-1\right)-\left\{1,\left(T T_{k}^{G P}-T T_{k}^{H O T}\right),\left(R_{k}^{G P}-R_{k}^{H O T}\right), F_{k}^{H O O}\right\}\right) \times\left\{\begin{array}{c}
-B_{k}^{-} \\
V O T_{k}^{-} \\
V O R_{k}^{-} \\
1
\end{array}\right\}
\end{gathered}
$$

As shown in Chapter 4, the ALMF estimation algorithm requires initial estimates for the parameters $K_{0}, B_{0}, V O T_{0}, V O R_{0}, P_{0}, q_{0}, Q_{0}, r_{0}$ and $R_{0}$ to recursively produce estimates of $B_{k}, V O T_{k}$ and $V O R_{k}$ for each time interval $k$. This is explained later in this chapter. As time evolves, the impact of the parameter initial values are expected to diminish, producing accurate estimates of $B_{k}, V O T_{k}$ and $V O R_{k}$ can be expected.

\subsection{Case Study}

A case study is presented in this section to demonstrate the use of the estimation process

discussed above. The case study objective consists of the estimation of the $B_{k}, V O T_{k}$ and $V O R_{k}$ parameters using the ALMF algorithm and the available ITS data considering different proxies for travel time reliability.

In particular, the analysis focuses on a 6.5 mile long corridor in the northbound section of I-95 in Miami, Florida. This section has implemented HOT lanes with variable pricing since December of 2008 and the population of motorists is expected to be to be familiar with the HOT operation. The corridor experiences recurrent congestion in the afternoon between 14:30 and 19:30 but such congestion never reaches the entrance of the HOT lanes. The corridor and the data gathered by ITS devices are managed by FDOT 
District 6 by means of traffic management software referred to as the SunGuide software. The corridor has in total six lanes where two of them are HOT lanes and the remaining four are GP. The HOT lanes are fully segregated from the GP lanes by plastic poles, thus once motorists join the HOT lanes they cannot leave it until the end of the HOT lanes has been reached.

Additionally, this section of I-95 has a number of point traffic detectors located every $0.3-0.5$ miles that collect traffic basic parameters every 20 seconds. The collected data is archived in SunGuide operational files. The toll collection system and associated data is managed by the Turnpike Authority. The general characteristics of the corridor are shown in Figure 5.1.

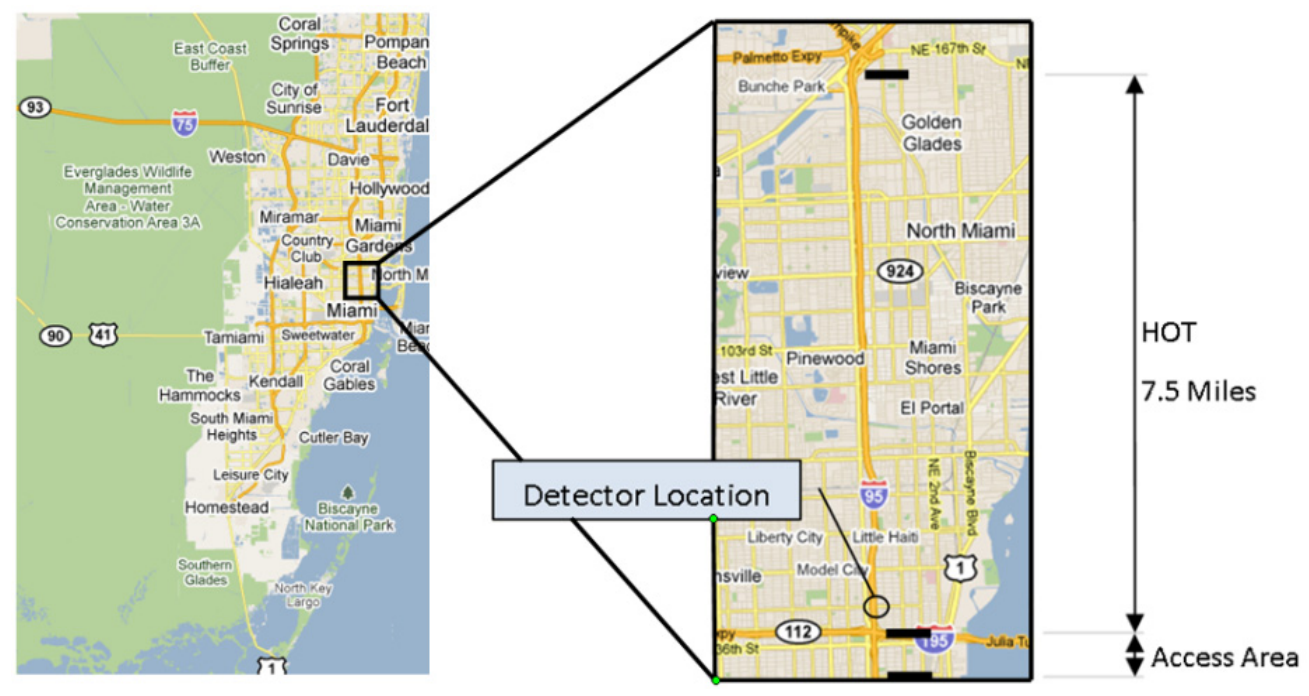

Figure 5.1: Study Area and HOT Layout

\subsubsection{Proportion of HOT Users}

HOT lanes can be used by single occupant vehicles paying the HOT lanes toll and by registered vehicles that are exempt of payment. The volumes of the HOT and GP users are acquired from a detector station located right after the gantry of the HOT of the system. 
This detector station normally never gets congested. The HOT and GP volumes are available at 20 seconds aggregation level, however a different aggregation level may be used for consistency with other system performance measures. The proportion of the HOT users is obtained by computing the ratio between the counts taken at the entrance of the HOT lanes versus the counts taken at the GP plus the HOT lanes. The counts at the GP lanes are adjusted by subtracting the counts of the vehicles exiting the freeway system on the ramps downstream of the HOT entrance (assumed 10\% of the total GP lanes throughput); however as will be shown later, the vehicles exiting the corridor can only affect the estimation of the HOT lanes bias, and subtracting the 10\% from the GP volumes is not necessary. Thus, in terms of the user sensitivity to time, varying measures such travel time, reliability, toll, and occupancy, the percentage of drivers leaving the system is irrelevant. Strictly speaking, the paying proportion of motorists cannot be directly extracted from the point traffic detectors. Instead, the actual number of motorists paying for joining the HOT lanes must be extracted from the system that manages the toll payments. Due to data availability issues, it was not possible to mine such a database, so a simplified approach was adopted. Based on the toll system manager, it is reasonable to assume that $95 \%$ of the total numbers of the HOT users actually pay, and the remaining percent corresponds to HOV vehicles. Figure 5.2 depicts a typical measured signal from the HOT proportion of users. In Figure 5.2, $\ln \left(1 / P_{k^{-}} 1\right)$ instead of the actual proportion is used because the Logit model needs to be linearized in order to use the ALMF. This is achieved by applying a logarithmic transformation to the Logit model such the difference between the GP and HOT utilities can be isolated in their linear form. 


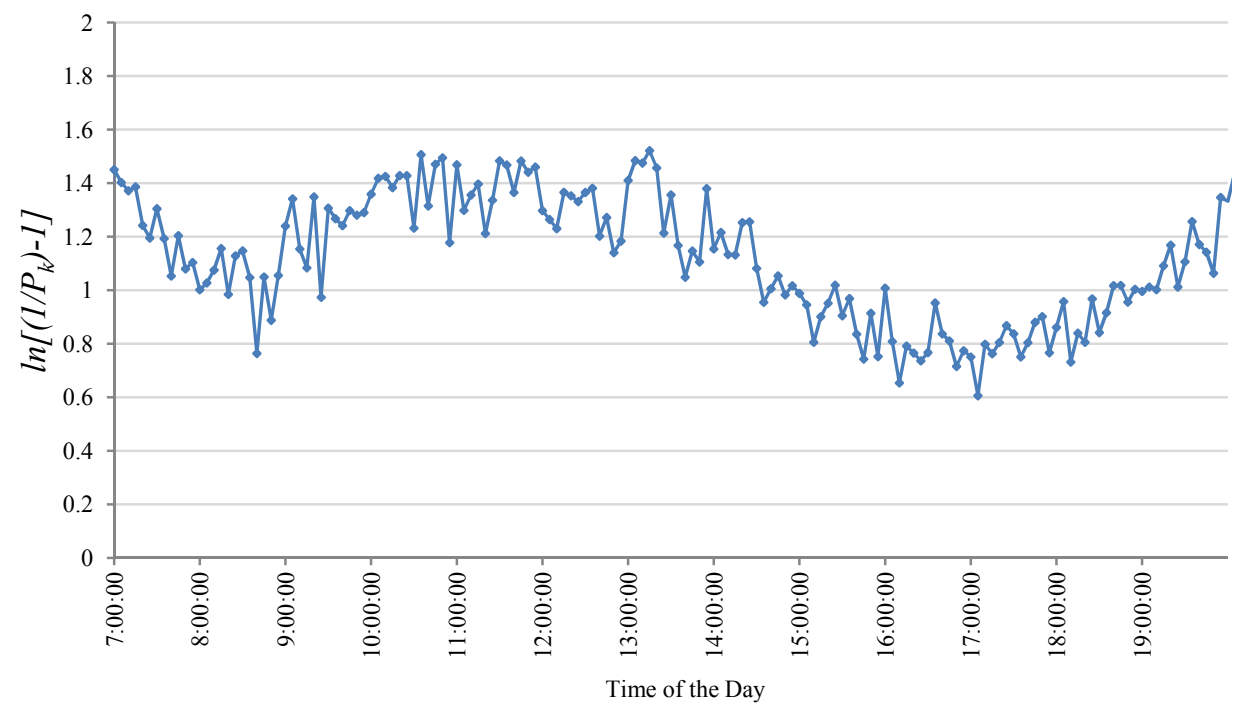

\section{Figure 5.2: Typical HOT Proportion of Users Signal $\ln \left(\left(1 / P_{k}\right)-1\right)$}

In terms of day to day variations of the HOT proportion of users, the average value, the standard deviation (STDV), and the coefficient of variation (CV) were computed as functions of time of the day; the result is depicted in Figure 5.3. As expected, the average proportion of HOT users significantly varies during the peak periods (07:00-10:00 and 16:00-20:00), while the day to day standard deviation of this measurement remains approximately the same throughout the day. This fact implies that the coefficient of variation also varies significantly during the peak demand periods, reflecting mainly time of day changes than day to day changes in the proportion of HOT users. The time of the day being the main source of variation of the proportion of HOT users, one may anticipate that the pricing policy in place may be responsible for the regularity observed in the HOT operation across the days. Despite this anticipation of linking the demand for the HOT lanes and the pricing policy, it is yet not clear the mechanism by which the pricing policy influences the throughput in the HOT lanes. 


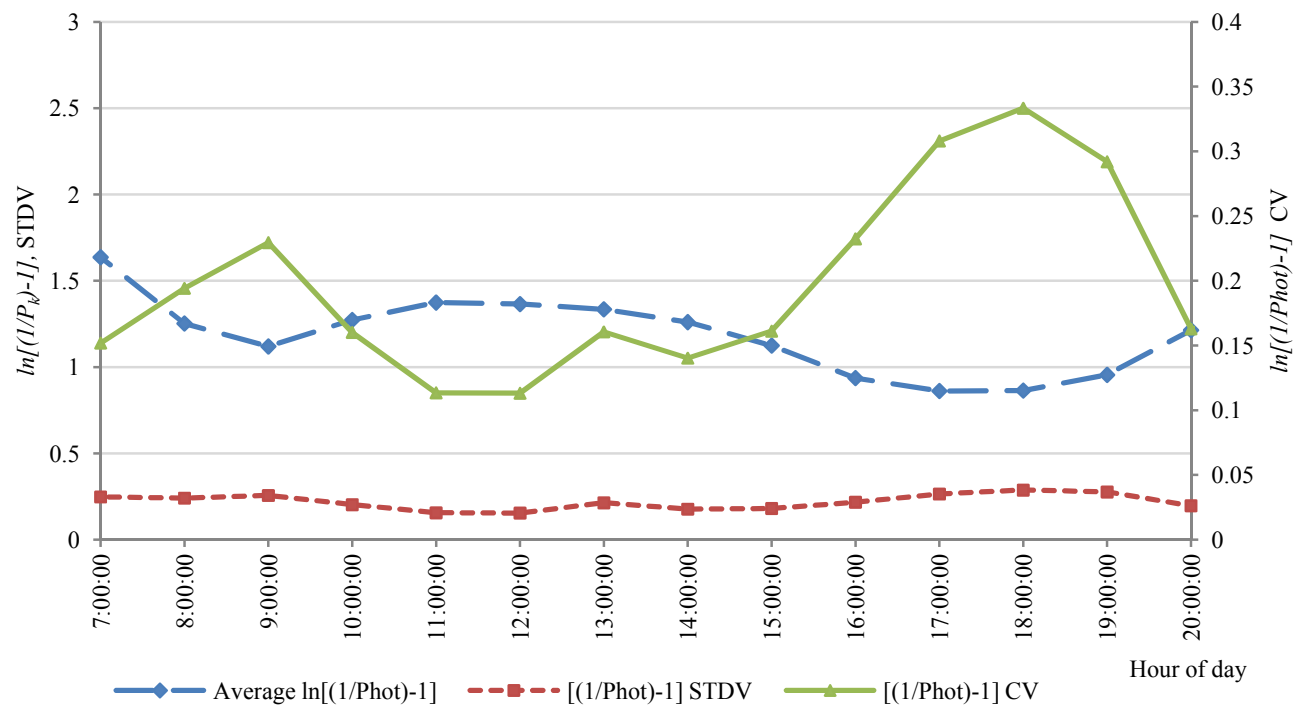

Figure 5.3: Daily Variation of HOT Proportion of Users Signal $\ln \left(\left(1 / P_{k}\right)-1\right)$

\subsubsection{Travel Time}

Travel time estimates for the HOT and GP lanes are available for every minute of the day from the SunGuide system. The travel time is indirectly estimated based on the speed reported by point traffic detectors at different locations of the system. The mid-point method is used to estimate the travel time between the starting and ending points of the section as it is done by the SunGuide software. Travel times are disseminated only for the GP lanes by means of dynamic message boards (DMS) located before the HOT lanes single gantry point and along the corridor. However, both HOT and GP travel time estimates are archived and available in the operational SunGuide archives. In terms of the travel time values used in this study, the conclusions from Chapter 3 were taken into account for estimating representative values. In particular, travel time was computed as the weighted average between the mean travel times during 60 days and the estimated travel time for the current day. The used weights were 0.75 for the historical information and 0.25 for the real time data. 
The typical average travel time difference between the GP and HOT lanes is shown in Figure 5.4 for as a function of time of day.

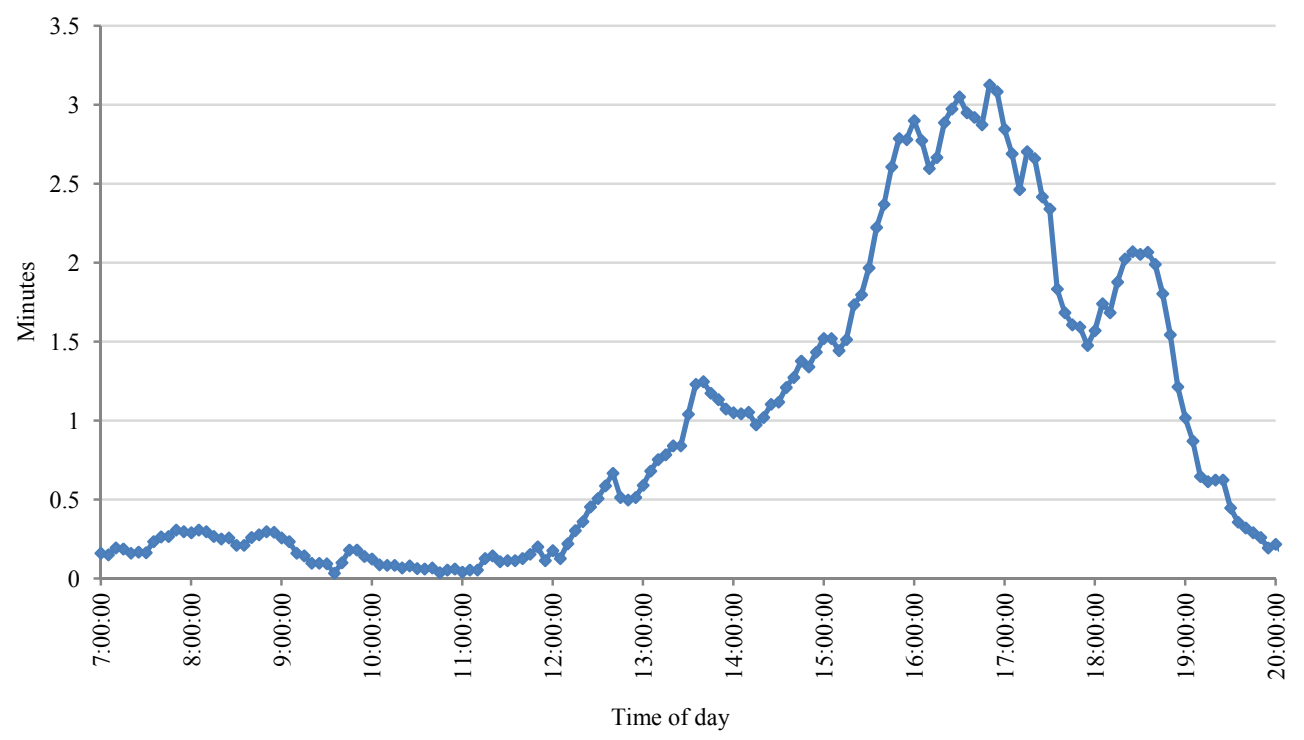

Figure 5.4: Typical GP and HOT Travel Time Difference

In terms of day to day variation of the travel time differences between the HOT and GP lanes, the average value, the standard deviation (STDV), and the coefficient of variation $(\mathrm{CV})$ were computed as function of the time of day; the results are depicted in Figure 5.5. Again, as expected, the mean travel time difference increases during the peak periods (07:00-10:00 and 16:00-20:00), however this increment tends to be significantly higher during the afternoon period which is also the peak period for the investigated direction. In the other hand, it can be noticed that the standard deviation of the mean tends to increase by the afternoon peak; nonetheless this increment is moderate compared to the travel time increment during this period. In the case of the coefficient of variation, it is observed that the travel time difference tends to be less variable in the afternoon than the morning period; this is explained by the fact that the variation in the mean travel time due to congestion is significantly higher than the day to day variability of the mean travel 
time samples. This regularity in the travel time pattern across the days is consistent with the pattern observed for proportion of the HOT users described before. Thus, it is logical to also link the travel time regularity with the pricing policy in place.

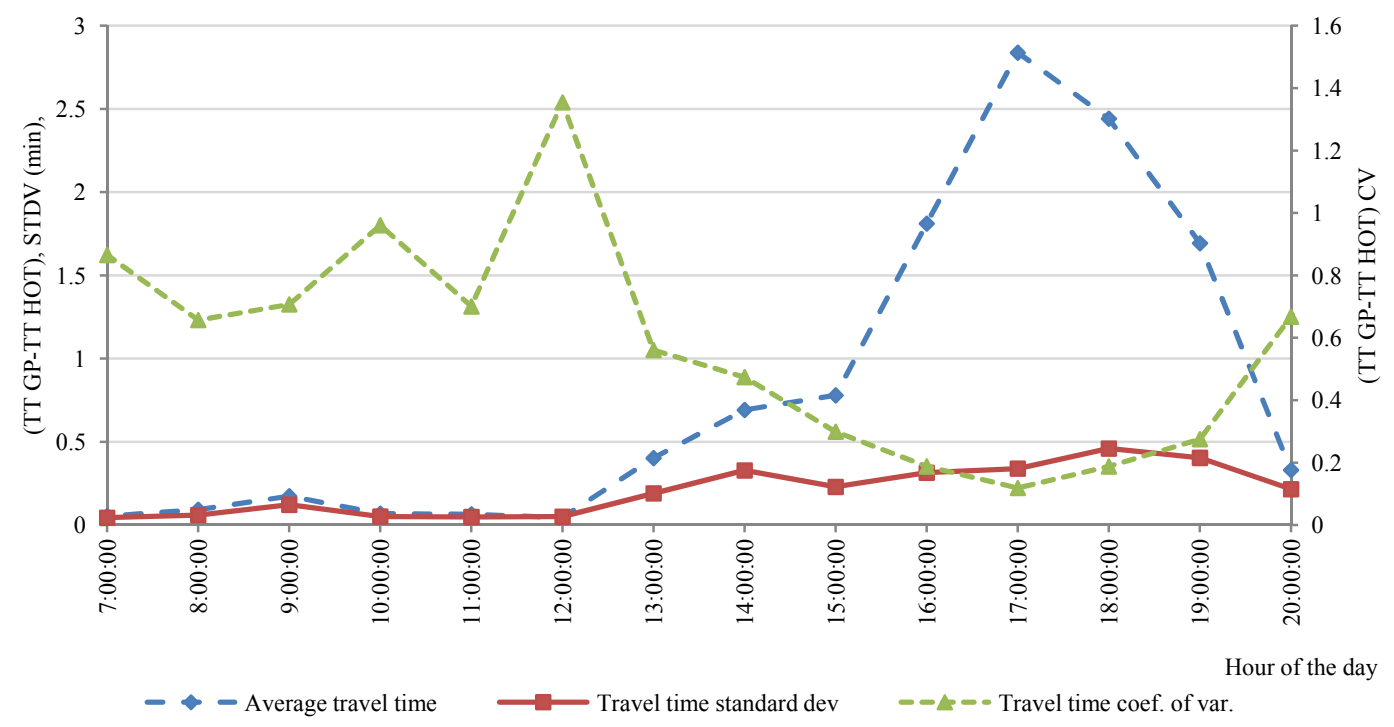

Figure 5.5: Travel Time Difference Day to Day Statistics

\subsubsection{Tolls}

Tolls in the HOT lanes are manually updated every 15 minutes with the objective of maintaining operational speeds in the HOT lanes that never go below a certain threshold and therefore maintain specific levels of service on the HOT lanes. The updated toll is obtained from a lookup table where the operator can select the toll that increases the speed in the HOT lanes given the actual conditions on the HOT and GP lanes. The toll for different times of the day and different days is archived in an Oracle database. Figure 5.6 shows an example of the typical tolls charged in a weekday along the day. 


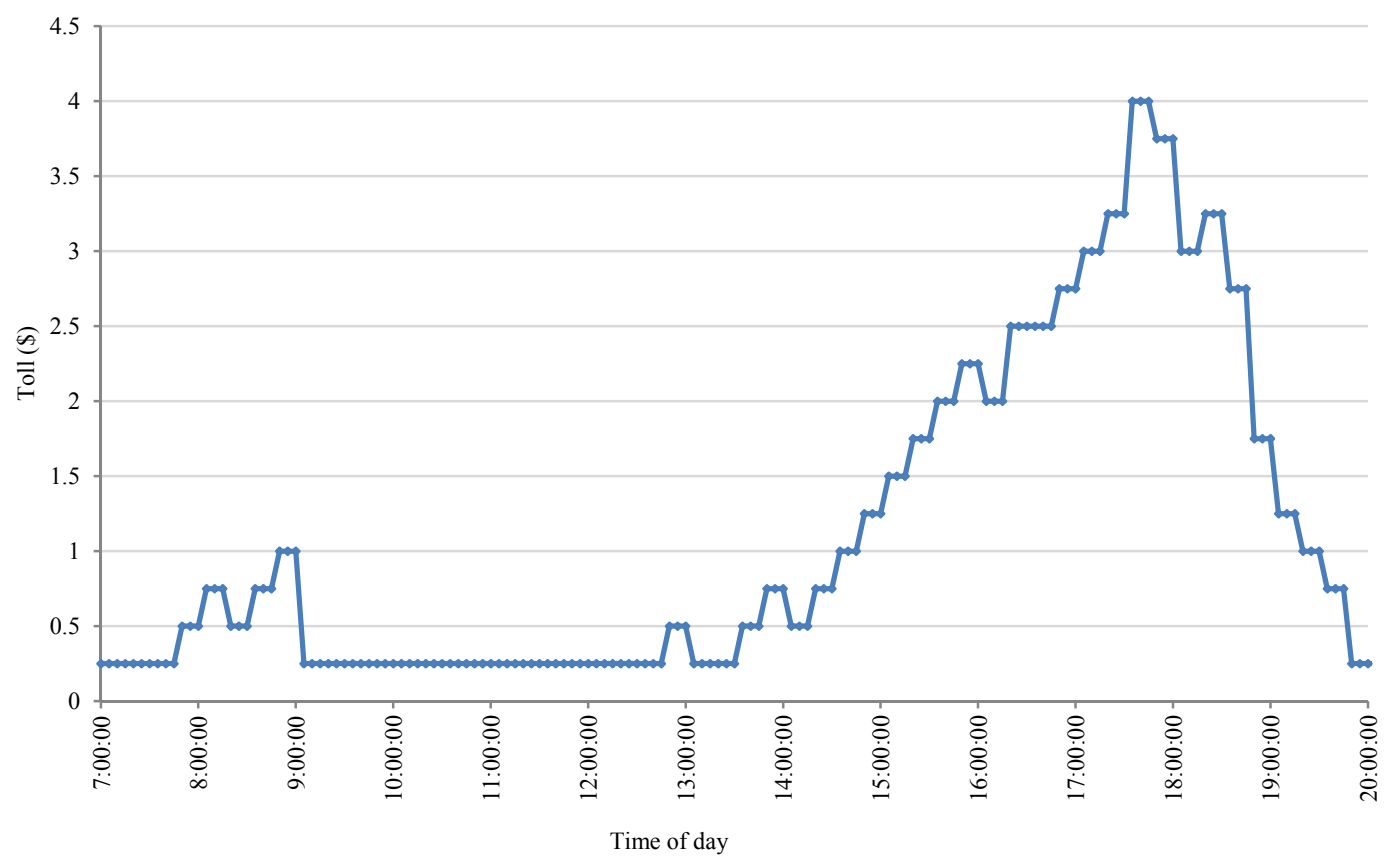

Figure 5.6: Typical Toll Values During Weekdays

In terms of the day to day variation of the toll; the average value, the standard deviation (STDV) and the coefficient of variation (CV) were computed for the available dataset as a function of the time of day; the results are shown in Figure 5.7. Again, consistently with the travel time patterns, it is observed that toll increases during the peak periods (07:00-10:00 and 16:00-20:00); and similar to the travel time case, the toll increment is significantly higher during the afternoon period. From the point of view of the day to day variability, the toll standard deviation tends to increase in both peak periods; yet the afternoon increment is higher than the values observed during the morning peak and particularly higher when compared to the day to day variation observed in the demand for HOT lanes and mean travel times. This toll pattern implies a coefficient of variation that remains relatively high during most parts of the day with values in the vicinity of two. This characteristic of the toll variation indicates that the toll 
changes significantly from day to day due to the attempt to control the demand for the HOT lanes on each particular day. This is somewhat contradictory because so far the characteristics of the priced facility (travel time and throughput, for instance) do not change significantly from day to day while toll values do. This may be indicative of the type of control that is being exercised by the pricing policy in place, which in this case seems to be better explained by the existence of thresholds in the toll that have the power to discourage users from joining the HOT. Those thresholds also seem to become active at certain times of day depending of the environment or driver expectations.

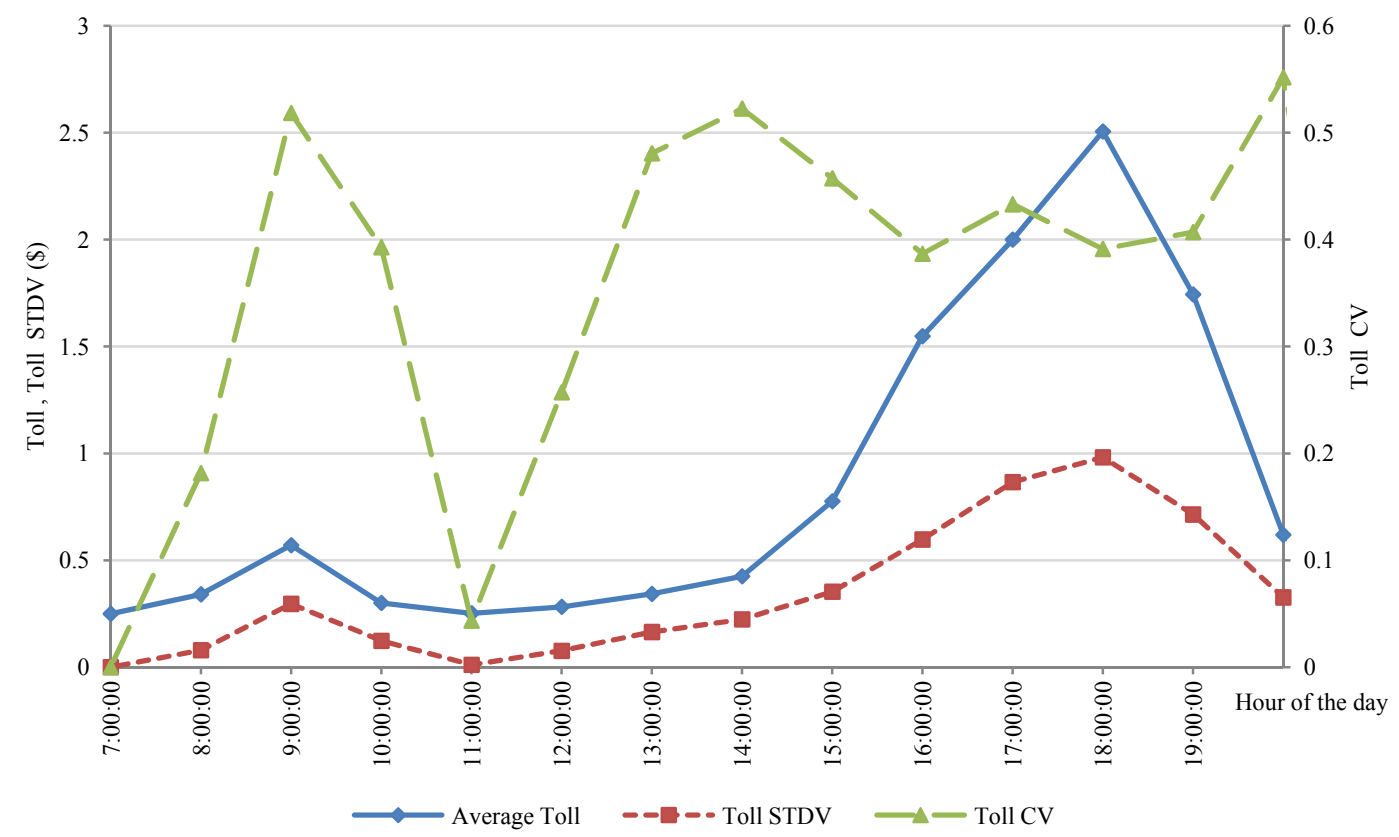

Figure 5.7: Toll Day to Day Statistics

\subsubsection{Travel Time Reliability}

Travel time reliability is slightly different from the other variables reviewed so far. Travel time reliability measures the level of consistency of travel conditions over time and it is measured by describing the distribution of travel times that occur over a substantial period of time. Thus, travel time reliability is a derived measure that actually accepts a 
wide range of operational definitions, as long as they describe the travel time temporal variation. In practice, travel time reliability can be computed for any time period based on the observed distribution of the travel times in the same period of time along several days.

As discussed in Chapter 3, there are several different operational definitions of travel time reliability and they are not necessarily consistent among themselves. Here, the different reliability definitions considered in Chapter 3 are used. However it was noticed that from all the travel time reliability metrics proposed in Chapter 3, only two of them, PTI $95^{\text {th }}$ and PTI $80^{\text {th }}$ showed consistent patterns with the observed proportion of HOT users. Figures 5.8 to 5.12 show the typical relationship between different reliability metrics and the observed proportion of the HOT users. It is observed that the variations of the Misery Index, the Buffer Index, and the Skew Statistic basically happen in a relatively flattened fashion, indicating that none of the proportion of the HOT users variability can be actually explained by these variables. In the case of the FOT 1.1 and FOT 1.25 , as mentioned in Chapter 3, there are not consistent trend of variation with the observed proportion of HOT users, a thus a rather fuzzy relationship is observed between FOT and HOT proportion. These variables will be set aside of the analysis due to their poor explanatory power. 


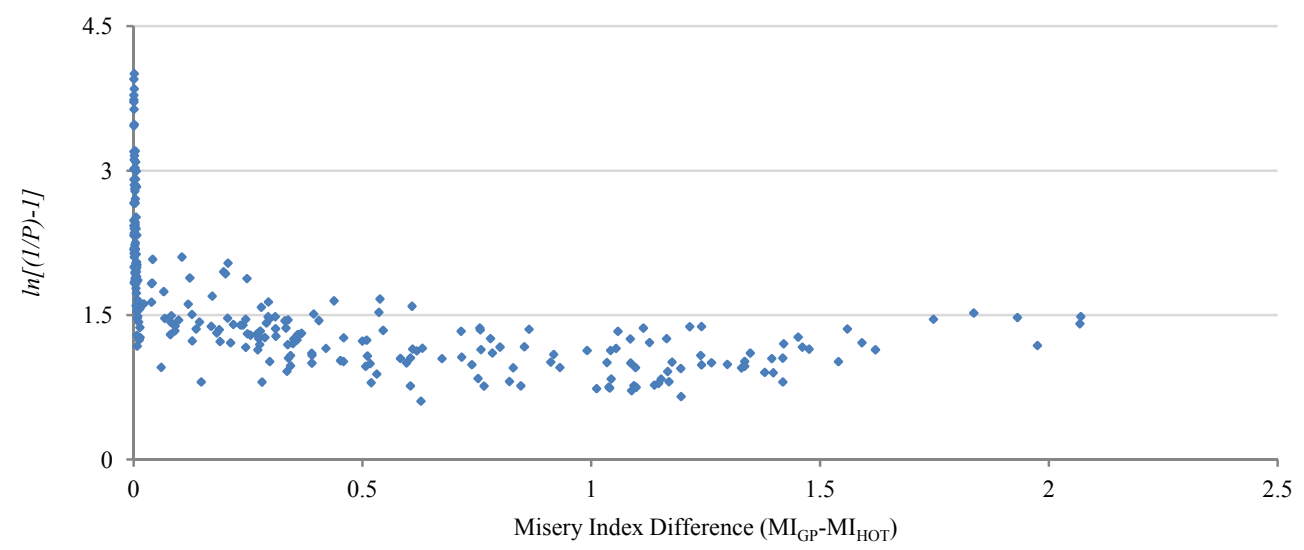

Figure 5.8: Relationship between HOT Users and the Misery Index Difference

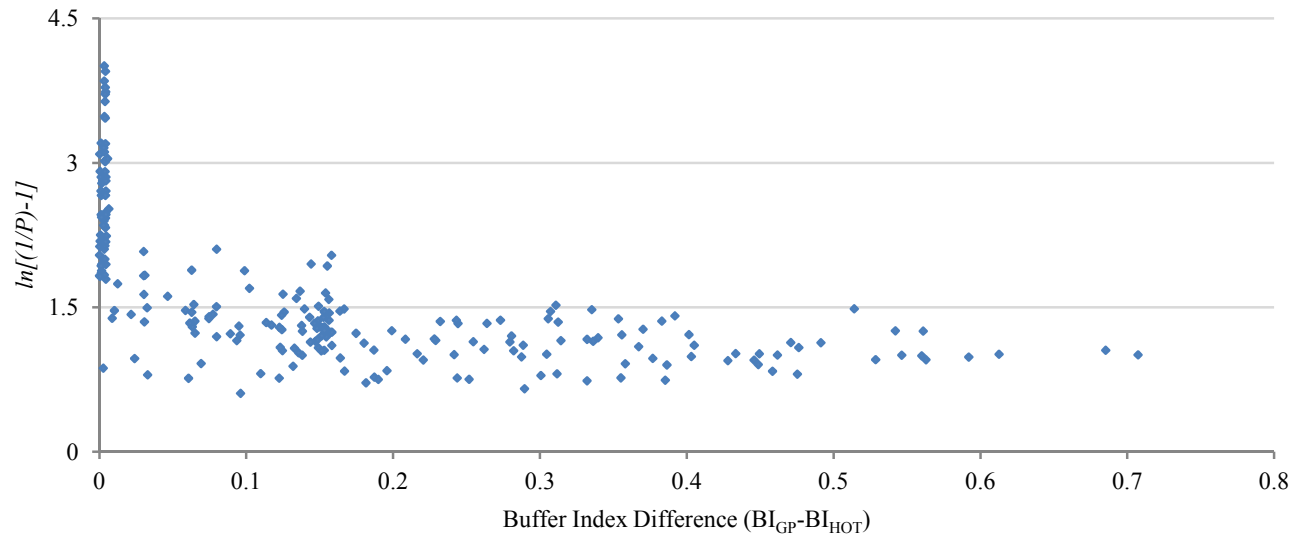

Figure 5.9: Relationship between HOT Users and the Buffer Index Difference

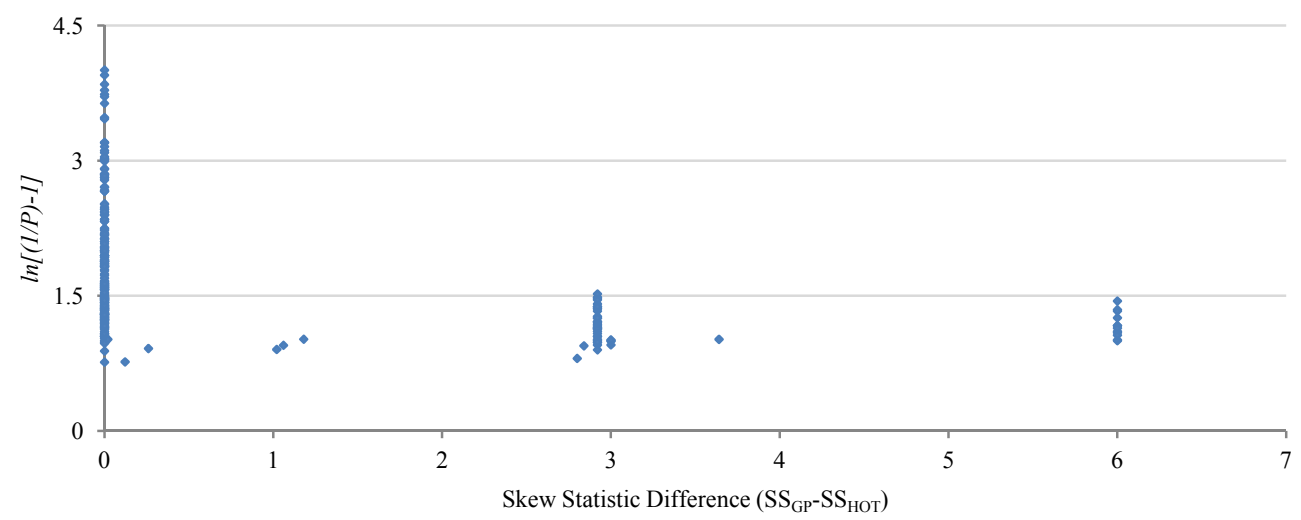

Figure 5.10: Relationship between Observed HOT Users and the Skew Statistic Difference 


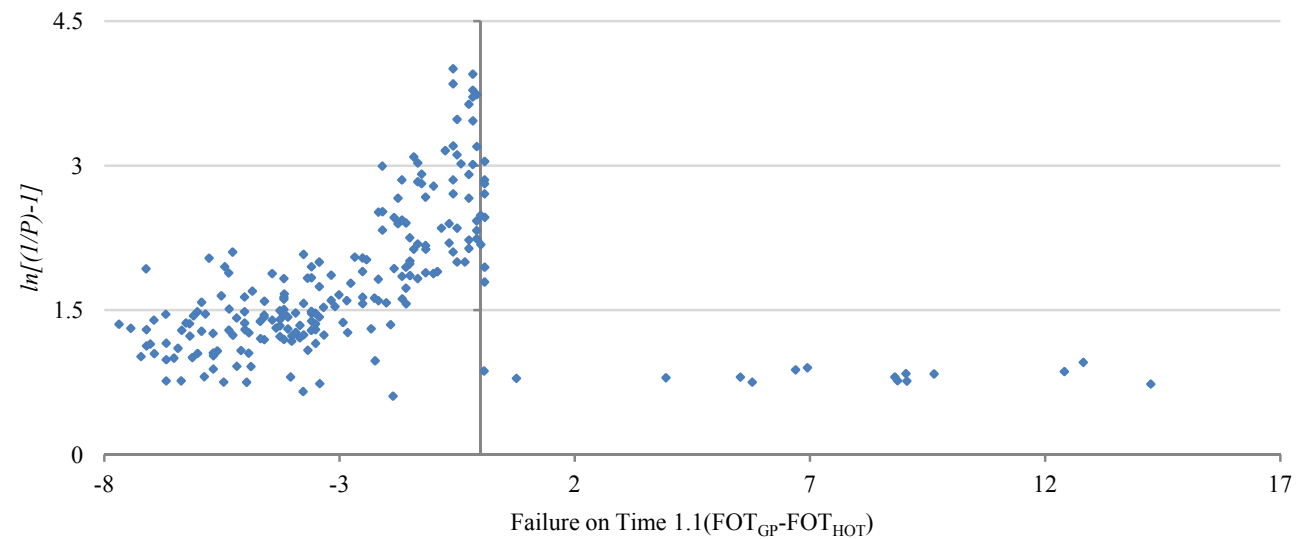

Figure 5.11: Relationship between Observed HOT Users and the Failure on Time 1.1 Difference

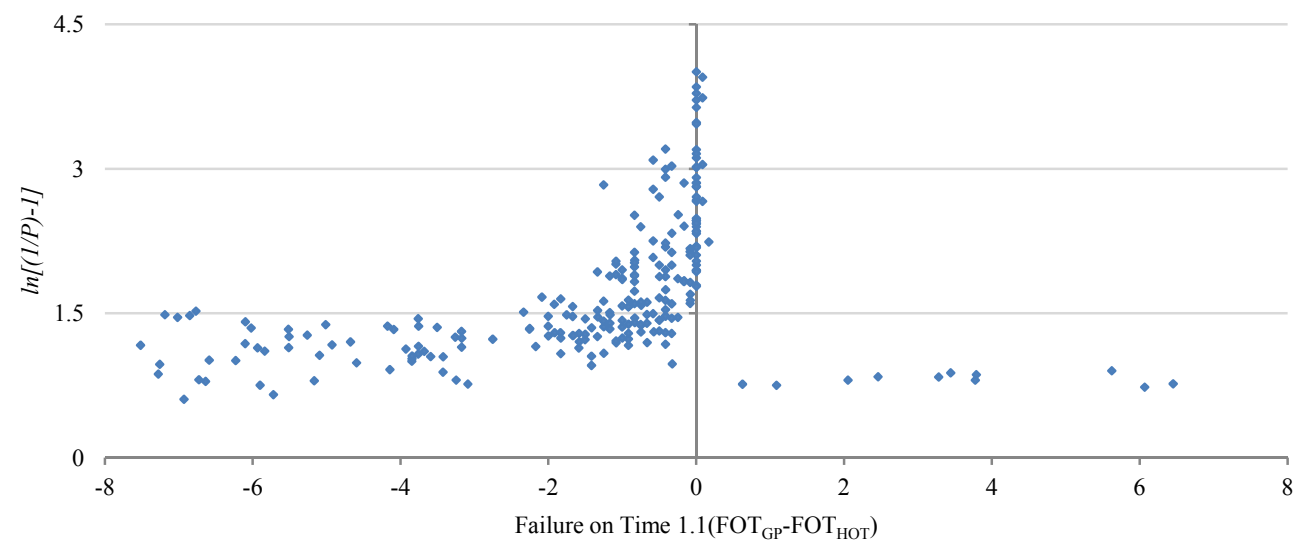

Figure 5.12: Relationship between Observed HOT Users and the Failure on Time

\subsection{Difference}

\subsubsection{Occupancy}

The occupancy of the HOT and GP lanes is acquired from detector stations located along the corridor under study. The HOT and GP occupancies are available at 20 seconds aggregation level, however a different aggregation level may be used for consistency with other system performance measures. The occupancy of the HOT lanes is obtained by computing the time of day weighted average of the stations contained in the HOT and GP 
lanes. Figure 5.13 shows an example of the typical occupancy difference between the HOT and GP lanes observed in a weekday as function of time of day.

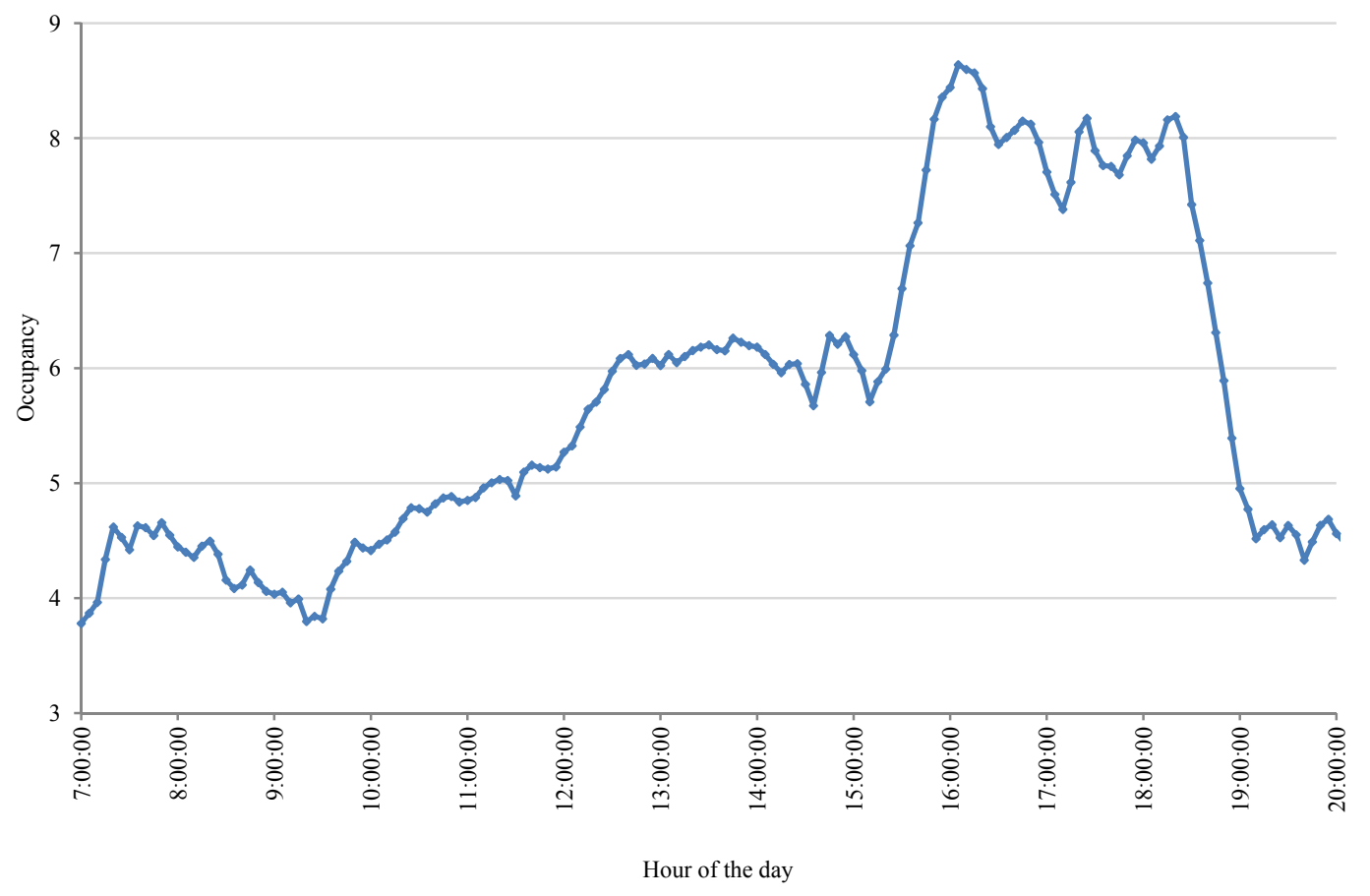

Figure 5.13: Typical Occupancy Difference Values During Weekdays

In terms of the occupancy daily variation, the average value, the standard deviation (STDV), and the coefficient of variation (CV) were computed for the available dataset as a function of the time of the day; the results are shown in Figure 5.14. It is observed that occupancy gradually increases as the day develop, with the afternoon the period of time having higher occupancy difference values between the HOT and GP lanes. From the point of view of day to day occupancy variability, it can be noticed that occupancy standard deviation is relatively small, tending to increase by both peak periods. Considering the relationship between the occupancy standard deviation and the occupancy mean as reflected by the coefficient of variation, two peaks corresponding to 
the highest occupancies during the morning and afternoon are observed, however those points are significantly different from the trend observed for the rest of the day as shown in the figure.

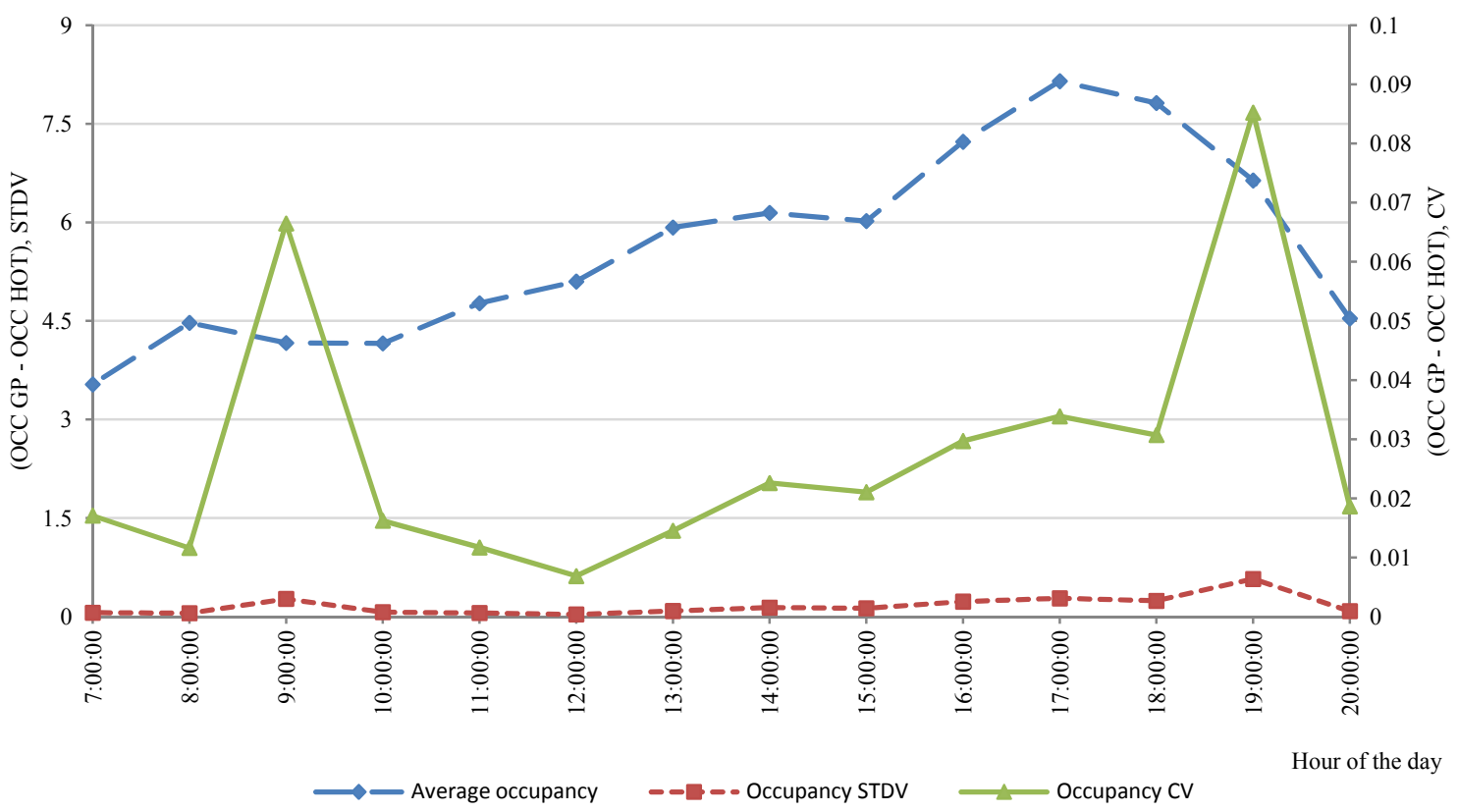

Figure 5.14: Occupancy Day to Day Statistics

\subsubsection{Data Selection}

The main objective of this study is to assess different sensitivities in the user behavior to changes in the freeway performance and their variability under different conditions. To capture the effects of the traffic conditions and traffic management strategies, a dataset was created by randomly selecting 45 days from the original dataset. A list of the specific day used is shown in the Appendix B. 


\subsubsection{Data Collinearity}

Filtering estimation techniques like the Kalman filter and its extension the AMLF algorithm are seriously affected by collinearity in the data. Collinearity arises as the explanatory variables used in the model move further and further away from orthogonality, which as in regression analysis, causes the estimates to become less reliable (high variance). In the limit, if the explanatory variables are linearly dependent, then the parameter estimates become indeterminate.

The presence of the collinearity can be ascertained by computing the correlation matrix of the regressors. It has been recommended in the literature that correlation coefficients greater than 0.5 , indicates the presence of data collinearity. Here, collinearity is checked among travel time, travel time reliability toll and occupancy. Figures 5.15 to 5.18 show the relationship between the travel time and toll, travel time and PTI $95^{\text {th }}$, travel time and PTI $80^{\text {th }}$, travel time and occupancy for a typical instance of the dataset.

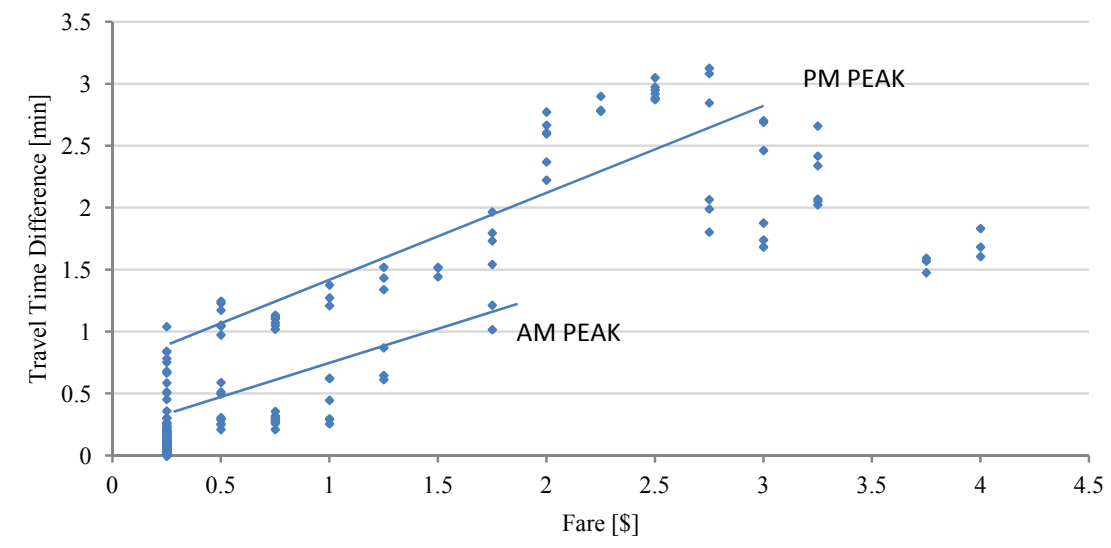

Figure 5.15: Typical Relationship between Travel Time and Toll 


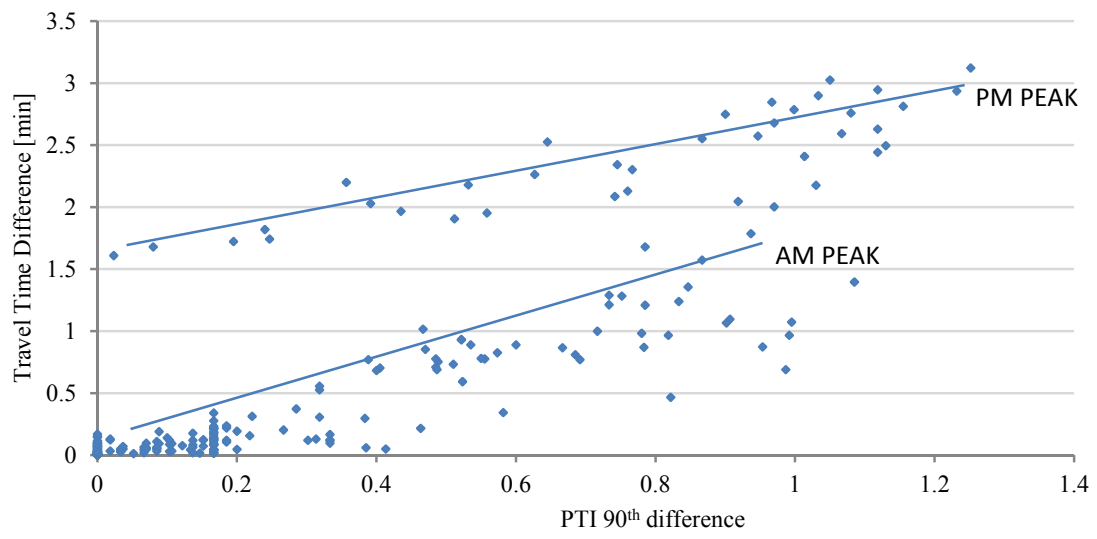

Figure 5.16: Typical Relationship between Travel Time and PTI $95^{\text {th }}$

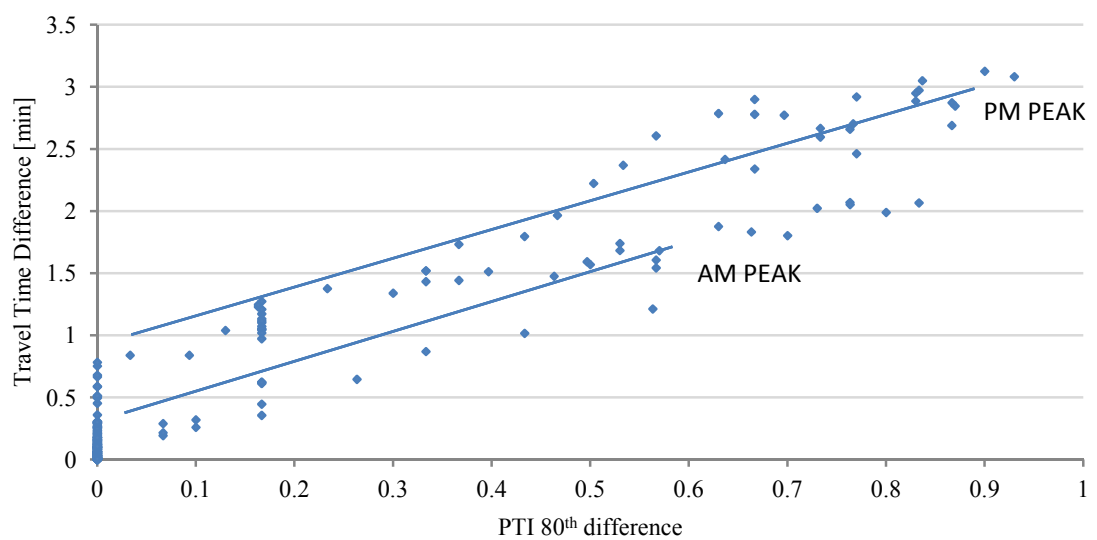

Figure 5.17: Typical Relationship between Travel Time and PTI $80^{\text {th }}$

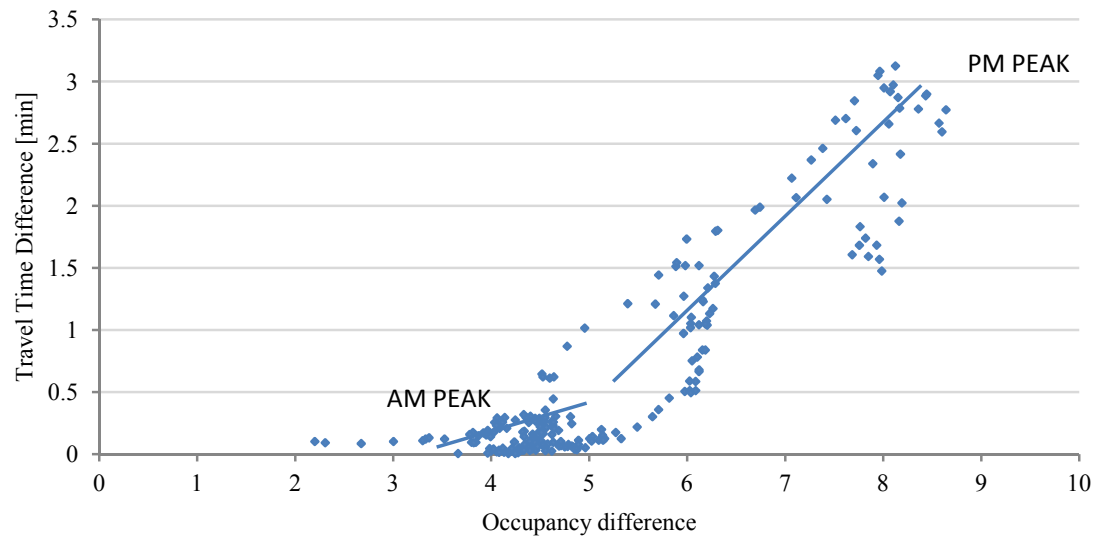

Figure 5.18: Typical Relationship between Travel Time and Occupancy 
It is observed that travel time, toll, PTI $95^{\text {th }}$, PTI $80^{\text {th }}$ and occupancy are strongly correlated during the AM and PM periods. This is an undesirable characteristic of the data used in this study because it constrains us from estimating joint models with more than one explanatory variable at the time. Table 5.1 shows the typical correlation factors between candidate explanatory variables. The observed magnitude of the correlation factors indicate that considering simultaneously two or more of these variables at the same time may result in the AMLF producing highly unreliable and, in the limit, indeterminate estimates of the parameters of interest. This possibility was further explored for several combinations of variables. The results were consistent with the recommendations in the sense that considering any combination of two or more of these variables result in the AMLF producing high variance estimates with no practical interpretation or application.

Table 5.1: Typical Correlation Factors

\begin{tabular}{|c|c|c|c|c|c|c|c|}
\hline & & \multicolumn{3}{|c|}{$\begin{array}{c}\text { Average } \mathrm{R}^{2} \text { Values Observed } \\
\text { During AM Period }\end{array}$} & \multicolumn{3}{|c|}{$\begin{array}{l}\text { Average } \mathrm{R}^{2} \text { Values Observed } \\
\text { During PM Period }\end{array}$} \\
\hline & & Fare & PTI $95^{\text {th }}$ & PTI 80th & Fare & PTI 95th & PTI $80^{\text {th }}$ \\
\hline Dataset 1 & $\begin{array}{l}\text { Travel } \\
\text { time }\end{array}$ & 0.81 & 0.81 & 0.82 & 0.82 & 0.87 & 0.83 \\
\hline
\end{tabular}

\subsubsection{Parameter Estimation}

The parameter estimation is performed using the ALMF algorithm together utilizing the selected ITS data described in the previous sections. For estimation purposes, from the point of view of the aggregation level, the most restrictive dataset is the proportion of HOT users because this information is available only at 5 minute aggregation level. Thus, all attributes (travel time, travel time reliability, and toll) are consistently aggregated at 5 minutes level. 
As shown before, there are high levels of correlation among the explanatory variables, this is an undesirable characteristic because it prevents the consideration simultaneously of more than one explanatory variable at a time. Further use of the ALMF demonstrated that including more than explanatory variable at a time produce estimates with unreasonable, unbounded variances that do not convey any information about the actual traveler's sensitivity at all. Given the former constraint, the estimation process is performed utilizing one explanatory variable at the time, so at least it would be possible to identify the performance measures that best explain the observed proportion of HOT users. Eventually, it will also be possible to estimate the ratios between the estimated parameters given that more than one parameter may be significant. The estimated models will in general have the form shown in Equation 5.6:

$$
z_{k}=\left(\begin{array}{ll}
1 & x_{k}
\end{array}\right)\left(\begin{array}{l}
\beta_{0} \\
\beta_{1}
\end{array}\right)+v_{k}
$$

Where $\beta_{0}$ is a time varying constant, $\beta_{1}$ is a time varying parameter for the explanatory variable $x_{k}$ and $z_{k}=\ln \left(\frac{1}{P_{k}}-1\right)$ is the measured signal at the entrance of the HOT lanes.

\subsubsection{Tuning the ALMF algorithm}

The use of the ALMF requires fine tuning of a number of parameters in order to produce reliable estimates. Among the parameters required to be fine-tuned, the most important ones are the LAP (explained below) and the initial values of the parameters $\beta_{0}(k=0), \beta_{1}(k=0)$. 
The LAP corresponds to the number of time periods that serve as a basis for collecting samples for estimating the unknown process and measurement noise statistics. That is the value of LAP determines how many prior periods are used to compute the covariance matrices utilized in the ALMF for the current period. The selection of the LAP value is performed iteratively until the variance of the estimates is minimized during the period of time of interest. Figure 5.19 shows the typical variance of the estimates $\beta_{0}$ and $\beta_{1}$ as function of the day for various LAP values. From this figure it is possible to recognize that because of the time-varying variance of the measurement signal, the variance of the estimates also vary accordingly. Particularly, when the measurement signal variance is high (typically for the AM period, with low SNR, and little information contained in the signal), the ALMF provides low reliable estimates regardless of the LAP value used. On the other hand, when the measurement signal variance is low (typically for the PM period, with high SNR, and information contained in the signal), it is possible to select a LAP value such that the ALMF algorithm can minimize the estimate variance providing an optimal solution. This characteristic of the measurement signal affects the variance of both estimates, $\beta_{0}$ and $\beta_{1}$, however is more significant in the case of $\beta_{1}$ because as shown later in this section, $\beta_{1}$ values are much smaller than their variance. 


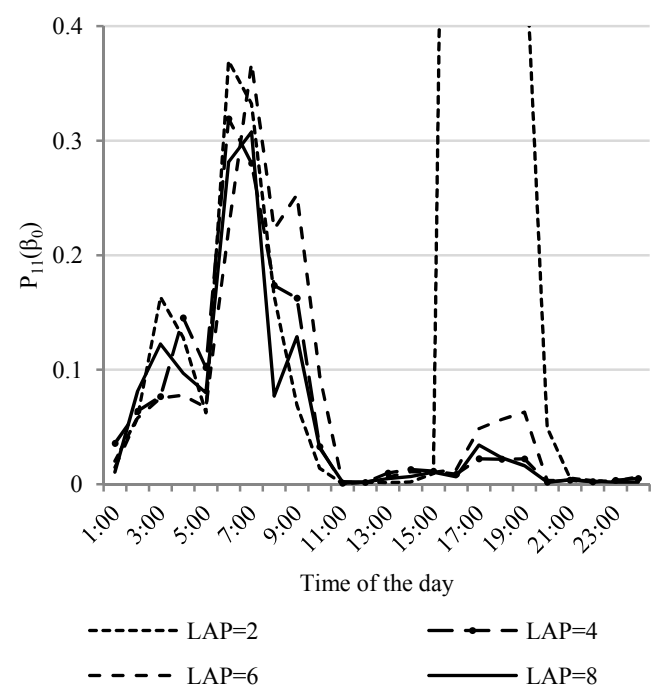

(a) $\beta_{0}$ estimate covariance

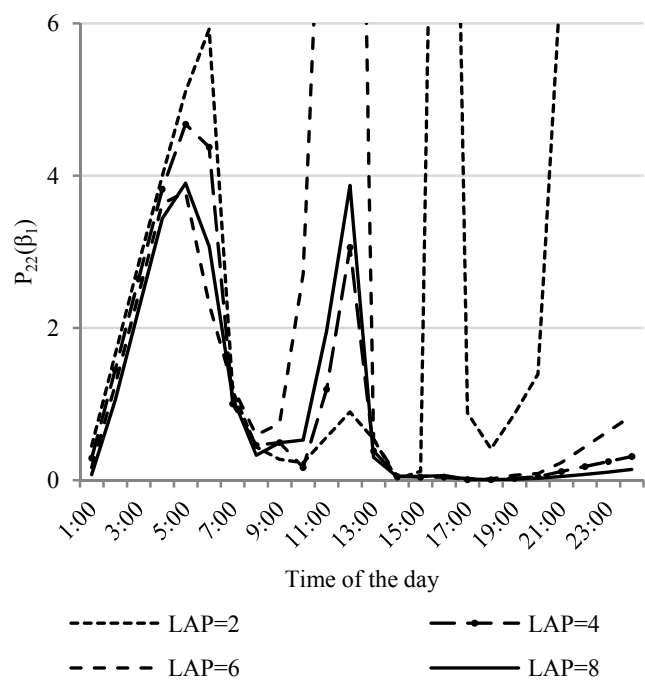

(b) $\beta_{l}$ estimate covariance

\section{Figure 5.19: Estimate Variance along the Day Different LAP values}

Figure 5.20 further shows the variance of the estimates $\beta_{0}$ and $\beta_{1}$ for selected hours.

The figure emphasizes the effect of the LAP value on the estimates variance. A summary of the LAP values used on each model are given in Table 5.2.

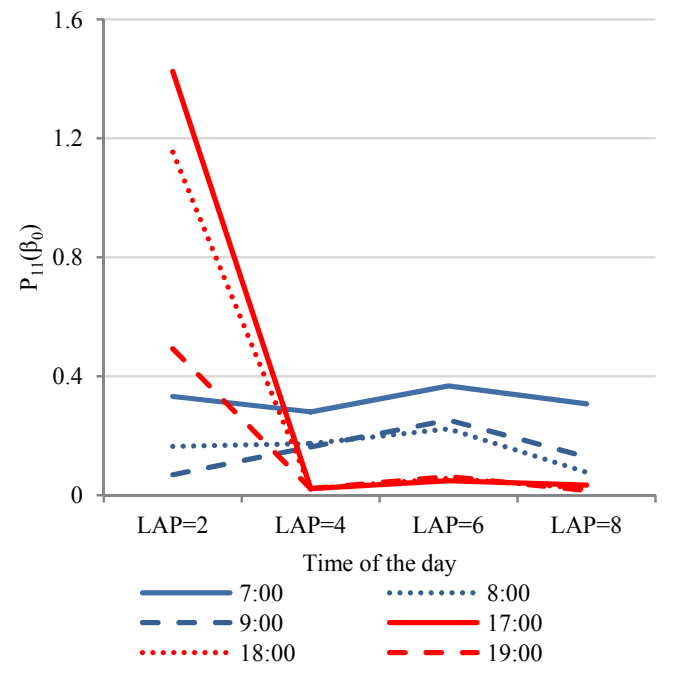

(a) $\beta_{0}$ estimate covariance

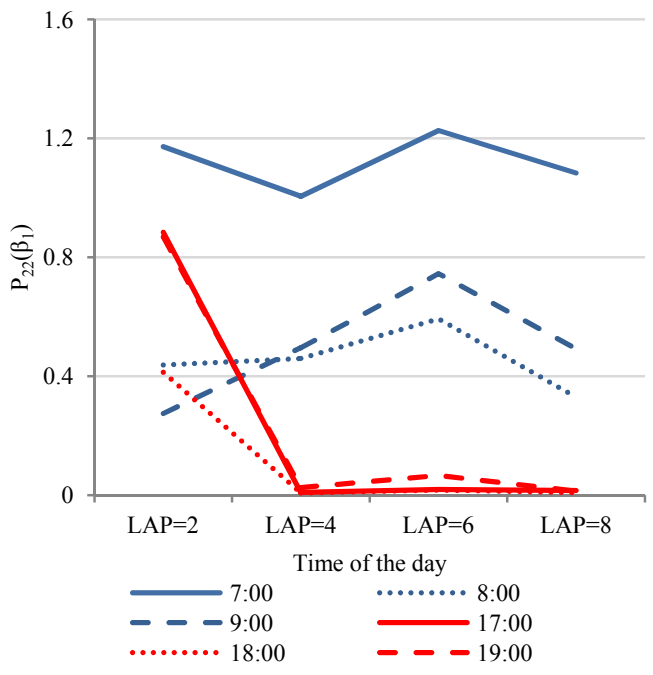

(b) $\beta_{1}$ estimate covariance

Figure 5.20: Estimate Variance Selected Hours for Different LAP values 


\section{Table 5.2: LAP Values Used on Each Estimation Problem}

\begin{tabular}{|c|c|c|}
\hline & Explanatory Variable & $\begin{array}{l}\text { LAP } \\
\text { Value }\end{array}$ \\
\hline Model 1 & Travel Time & 8 \\
\hline Model 2 & $\begin{array}{l}\text { Planning Time Index }\left(95^{\text {th }}\right. \\
\text { percentile })\end{array}$ & 8 \\
\hline Model 3 & $\begin{array}{l}\text { Planning Time Index }\left(80^{\text {th }}\right. \\
\text { Percentile })\end{array}$ & 8 \\
\hline Model 4 & Toll & 6 \\
\hline Model 5 & Occupancy & 6 \\
\hline Model 6 & Travel Time and Toll & 8 \\
\hline Model 7 & $\begin{array}{c}\text { Planning Time Index ( } 95^{\text {th }} \\
\text { percentile) and Toll }\end{array}$ & 8 \\
\hline Model 8 & $\begin{array}{l}\text { Planning Time Index ( } 80^{\text {th }} \\
\text { Percentile) and Toll }\end{array}$ & 8 \\
\hline
\end{tabular}

In Chapter 4 it was found that the initial values of the model parameters are also of utmost importance to accurately estimate the parameters in the model. In order to provide a good approximation to the initial values, a regression analysis (described below) was performed considering the entire period of interest (AM and PM), and the resulting estimates were used to feed to the ALMF algorithm with initial estimates and for comparison purposes as well. Figure 5.21 shows the typical regression analysis between $\ln \left[\left(1 / P_{k}\right)-1\right]$ and the travel time difference between GP and HOT lanes, for a typical day in the AM and PM periods. It can be seen that during the AM period, the users exhibit a higher sensitivity to the travel time than during the PM period (5.44 and 0.22 respectively). As described above, the values from the AM period regression are used as initial estimates $\left(\beta_{0}(k=0)=-5.44\right.$ and $\left.\beta_{1}(k=0)=2.49\right)$ in the ALMF algorithm while the values from the PM period regression are used as a reference to compare the estimates from the ALMF for the same period. 


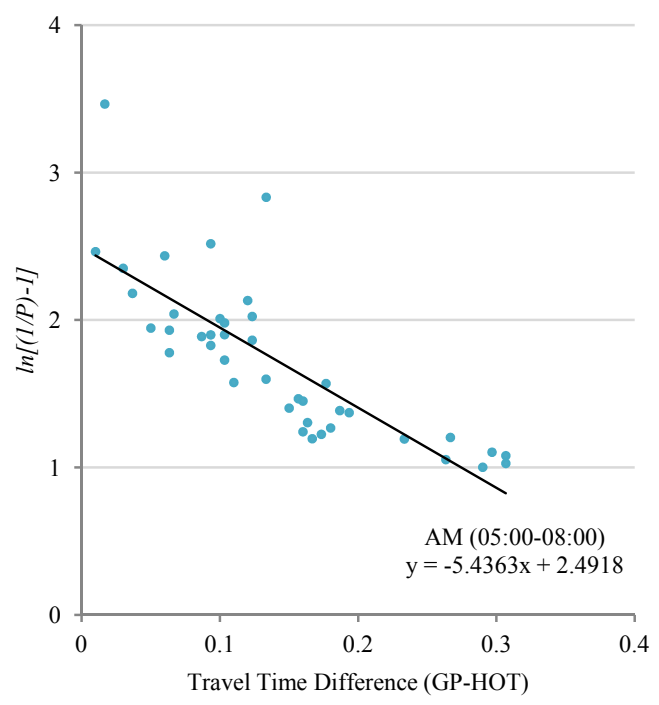

(a) AM Regression

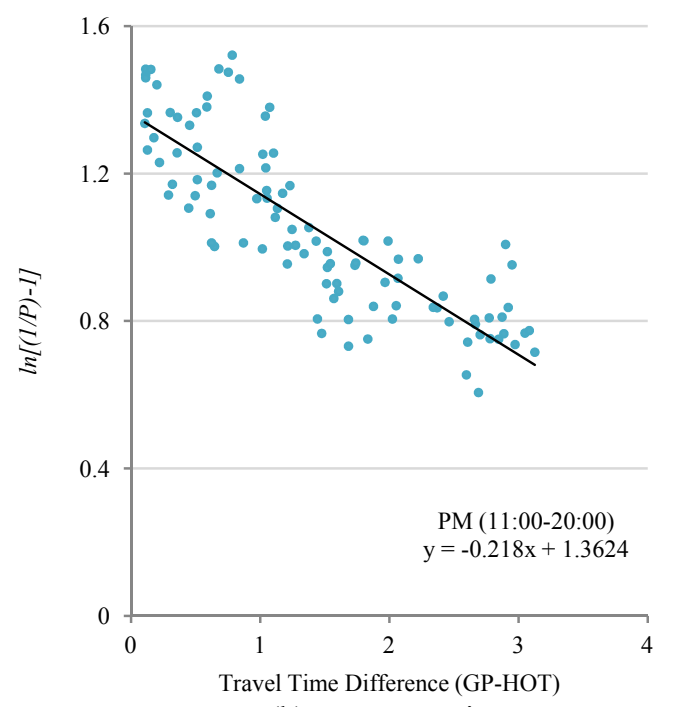

(b) PM Regression

\section{Figure 5.21: AM and PM Regression Analysis between the Observed signal and Selected Explanatory Variables}

\subsubsection{Time Varying Estimates}

The application of the ALMF algorithm together with the values of LAP and the initial values for the estimates allow the generation of time-varying estimates for $\beta_{0}$ and $\beta_{1}$.

Figure 5.22 shows the typical profile of the $\beta_{0}$ estimate while Figure 5.23 shows the typical profile of $\beta_{1}$ estimate when the difference in travel time is used as the explanatory variable. Using other explanatory variables, such as the ones considered in model 2, 3, 4 and 5, produce similar patterns but with different variance in the estimates. These results are shown in Figure 5.24 and Figure 5.25 for PTI $95^{\text {th }}$ and Figure 5.26, and Figure 5.27 for PTI $80^{\text {th }}$, Figure 5.28 and Figure 5.29 for Toll, Figure 5.31 and Figure 5.32 for occupancy respectively.

In the case of Model 1 that uses the travel time to explain the variations of the proportion of HOT users, the following observations are possible: 
- The time varying values of $-\beta_{0}$ follow a pattern similar to the observed pattern of the observed proportion of HOT users along the day. This is in contrast to the observed pattern of $\beta_{1}$ which tend to stay mostly around two values (one value for the AM period and one value for the PM period)

- While the values of $-\beta_{0}$ during normal business hours ( $06: 00$ to $\left.20: 00\right)$ tend to vary moderately during the day, the values of $\beta_{1}$ vary dramatically between the $\mathrm{AM}$ and PM periods. In the first case (with $\beta_{0}$ ), the results indicate that the bias towards the use of the HOT lanes is relatively constant throughout the day and changes to this bias are only observed late during the night to justify the use of the HOT lanes even though no differences in travel time between HOT and GP lanes can be observed. Similar observation can be made for Model 2 to Model 5 respectively. In the second case (for $\beta_{1}$ ), the dramatic changes in the sensitivities to the travel time can be explained by the fact that in terms of proportion of HOT users, the AM variation and the PM variation are relatively similar while the difference in travel time is quite different (PM travel time difference is higher than AM travel time difference). Thus, the sensitivity during the AM period has to be higher than the sensitivity in the PM period.

- In the non-congested periods, where no differences in travel time are observed, the variability of $\beta_{1}$ increases significantly due to the lack of difference in travel time between GP and HOT lanes. Thus, obviously in the non-congested periods, $\beta_{1}$ poorly explains the variation of the proportion of HOT users. Similar observation can be made for Model 2 to Model 5 respectively. 


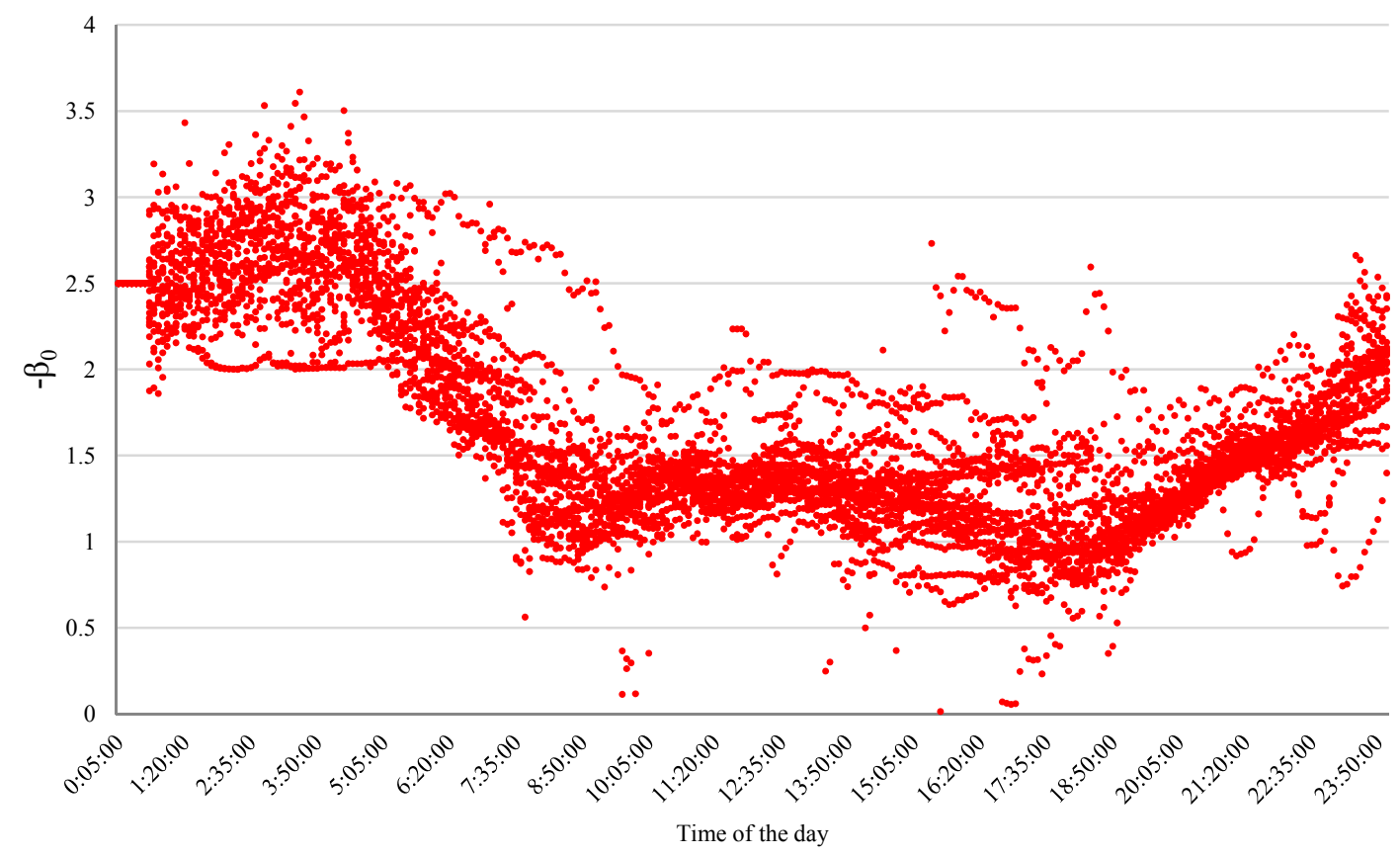

Figure 5.22: Time Varying Values of $-\beta_{0}$ Travel Time

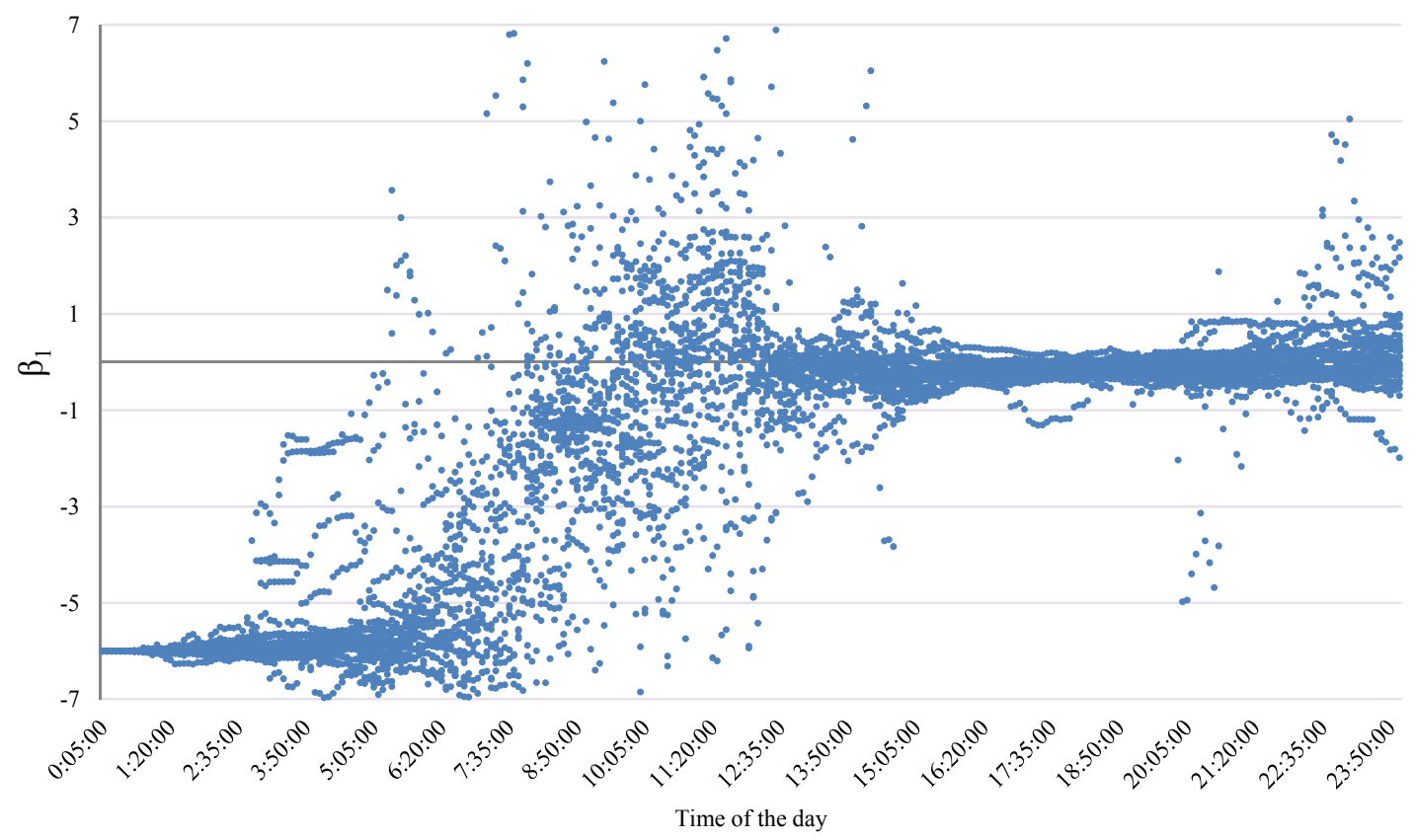

Figure 5.23: Time Varying Values of $\beta_{1}$ Travel Time 
In the case of Model 2 that uses the PTI $95^{\text {th }}$ to explain the variations of the proportion of HOT users, in addition to what was mentioned above, the following observations are possible:

- The estimation of $\beta_{1}$ is only possible in those periods where actual differences between PTI $95^{\text {th }}$ in the GP and HOT lanes do exist. The fact that the AMLF provides a constant value during early hours is because no information can be extracted from the data (due to high variance in the measurement data), the ALMF keeps the initial value provided as the best parameter estimate (estimate cannot be updated using updated measurements)

- Similar comment made for Model 1 is repeated here to justify the increase in the variance of the estimate in those periods of time where small differences between the PTI $95^{\text {th }}$ in the GP and HOT exists (06:00-12:00). However, significant reductions in the estimate day to day variability are observed with more significant PTI 95 differences between GP and HOT lanes. In the case of Model 3 that uses the PTI $80^{\text {th }}$ to explain the variations of the proportion of HOT users, similar comments to those provided for Models 1 and 2 apply. Nevertheless, it is worth noticing that using the PTI $80^{\text {th }}$ as explanatory variable produces a $\beta_{0}$ pattern that actually exhibits the lowest variability among the three models tested (See Figure 5.26). The value of $\beta_{1}$ also seems to be better defined from Figure 5.27, possibly indicating that the PTI $80^{\text {th }}$ percentile can explain more by itself compared to other investigated variables. 


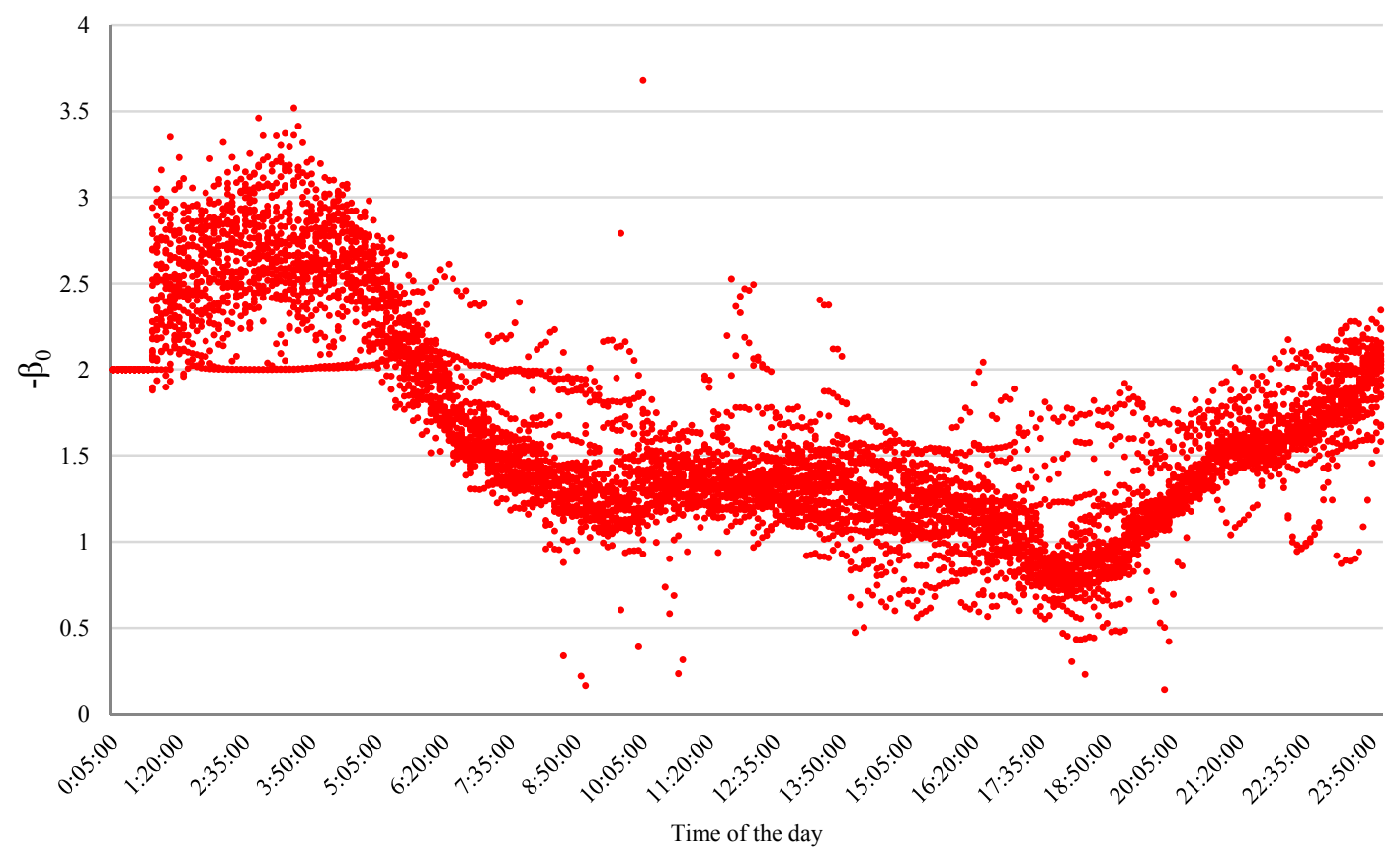

Figure 5.24: Time Varying Values of $-\beta_{0}$ PTI $95^{\text {th }}$

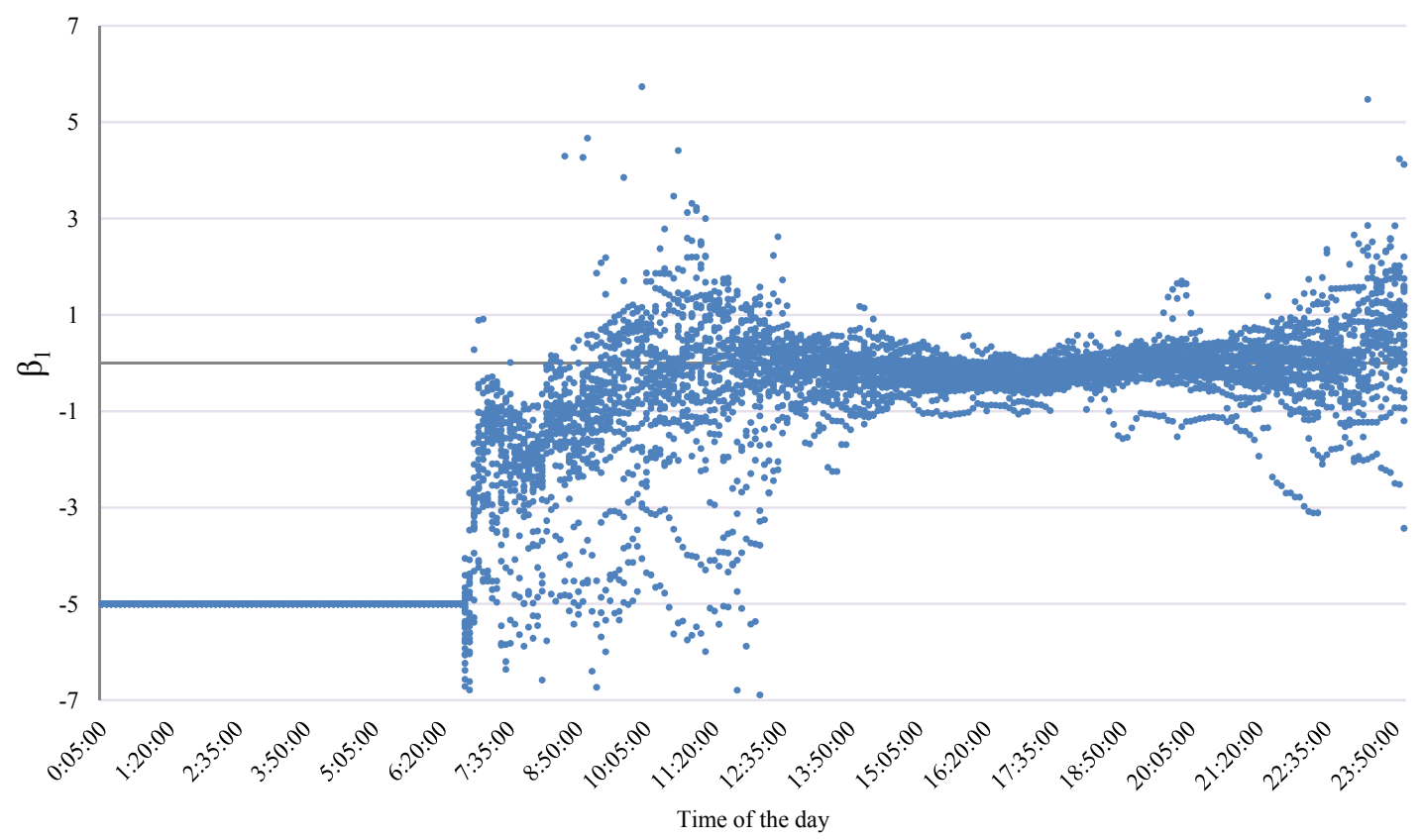

Figure 5.25: Time Varying Values of $\beta_{1}$ PTI $95^{\text {th }}$ 


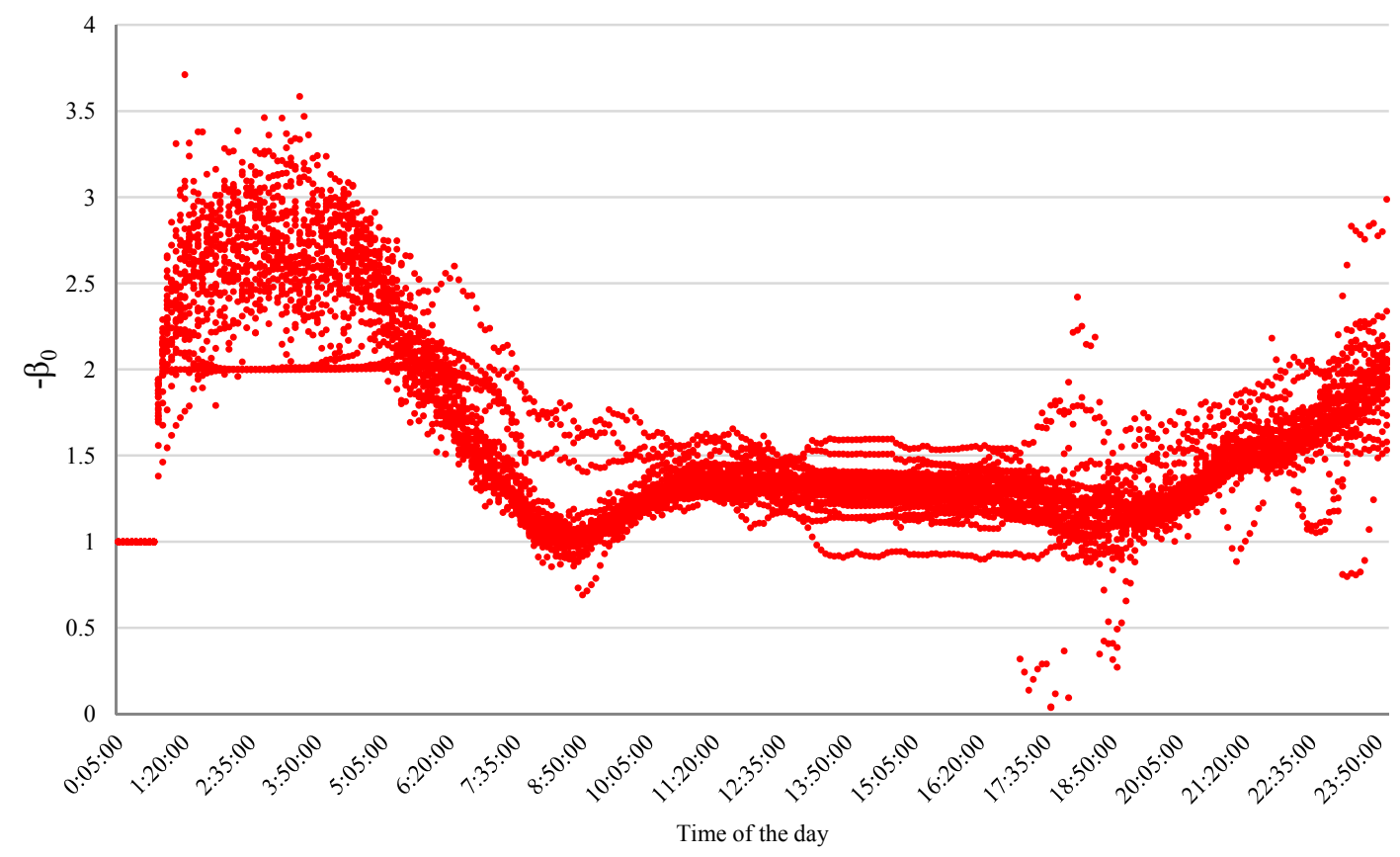

Figure 5.26: Time Varying Values of $-\beta_{0}$ PTI $80^{\text {th }}$

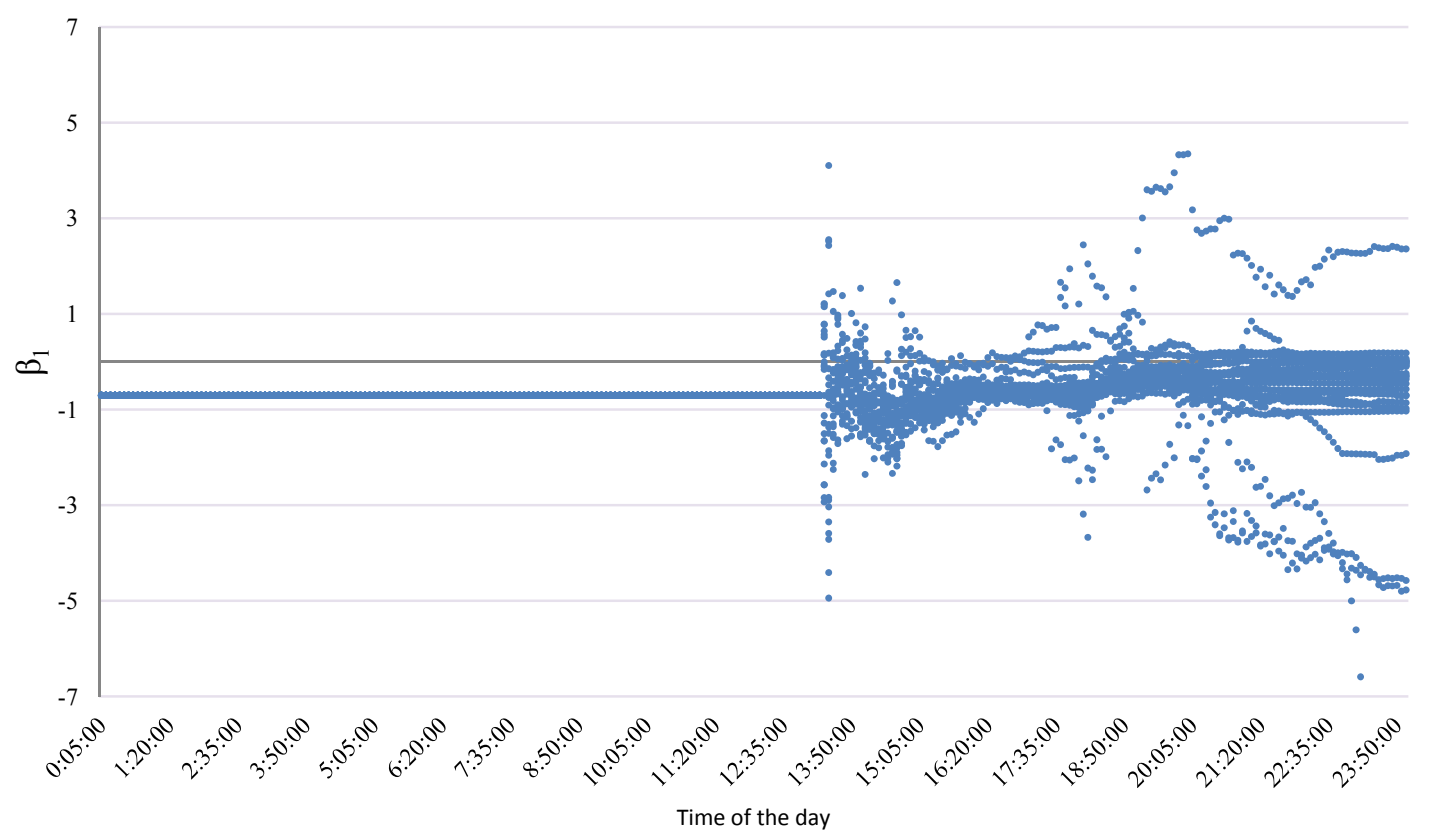

Figure 5.27: Time Varying Values of $\beta_{1}$ PTI $80^{\text {th }}$ 
In the case of Model 4 that uses the toll to explain the variations of the proportion of HOT users, the following observations are possible:

- First, the estimates of $-\beta_{0}$ show the highest variability among all the used models. Second, the estimates of $\beta_{1}$ indicate that there is no functional relationship between HOT demand and the toll charged during the congested periods.

- In the case of the bias parameter, the result is consistent with what was noticed in previous sections where the high day to day variability of the toll was highlighted. In fact, if systematically day by day, no functional relationship is observed between the toll and the HOT demand, the only way to fit the linear model is by means of adjusting the values of the parameter $\beta_{0}$. Thus, the observed variability of the estimated bias is a consequence of the high toll day to day variability and the lack of this parameter to serve as explanatory variable of the observed proportion of HOT users.

- In the case of the parameter for the toll, Figure 5.30 shows the relationship existing between the proportion of HOT users and the tolls charged for a typical day in the dataset. In this figure, the tolls have been divided depending on the period of the day when they were charged. A detailed examination of the toll data indicates that despite the apparent correlation between the demand for the HOT lanes and the toll, when the data is examined by period of the day, such correlation diminishes significantly (see the correlation factors). 


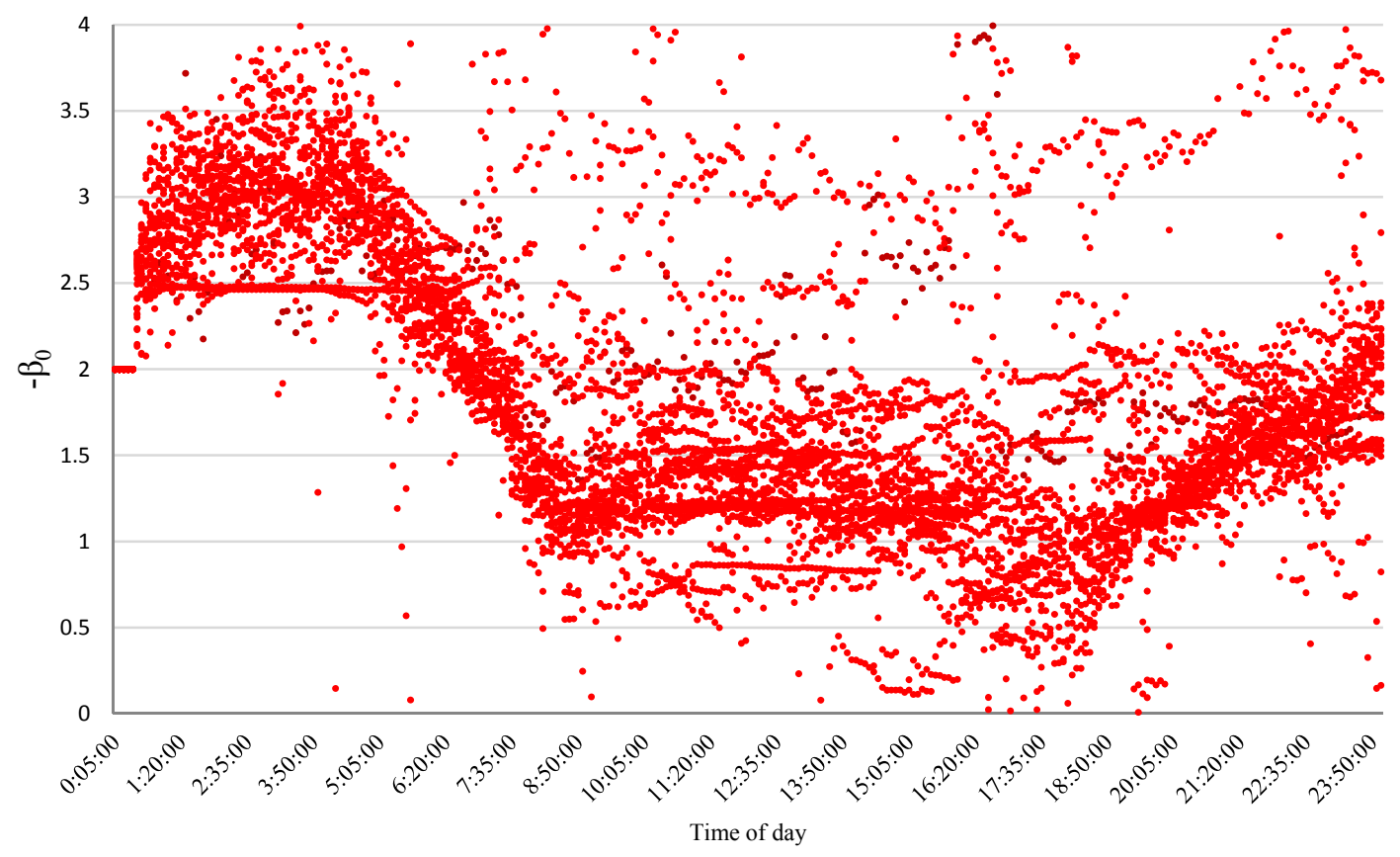

Figure 5.28: Time Varying Values of $-\beta_{0}$ Toll

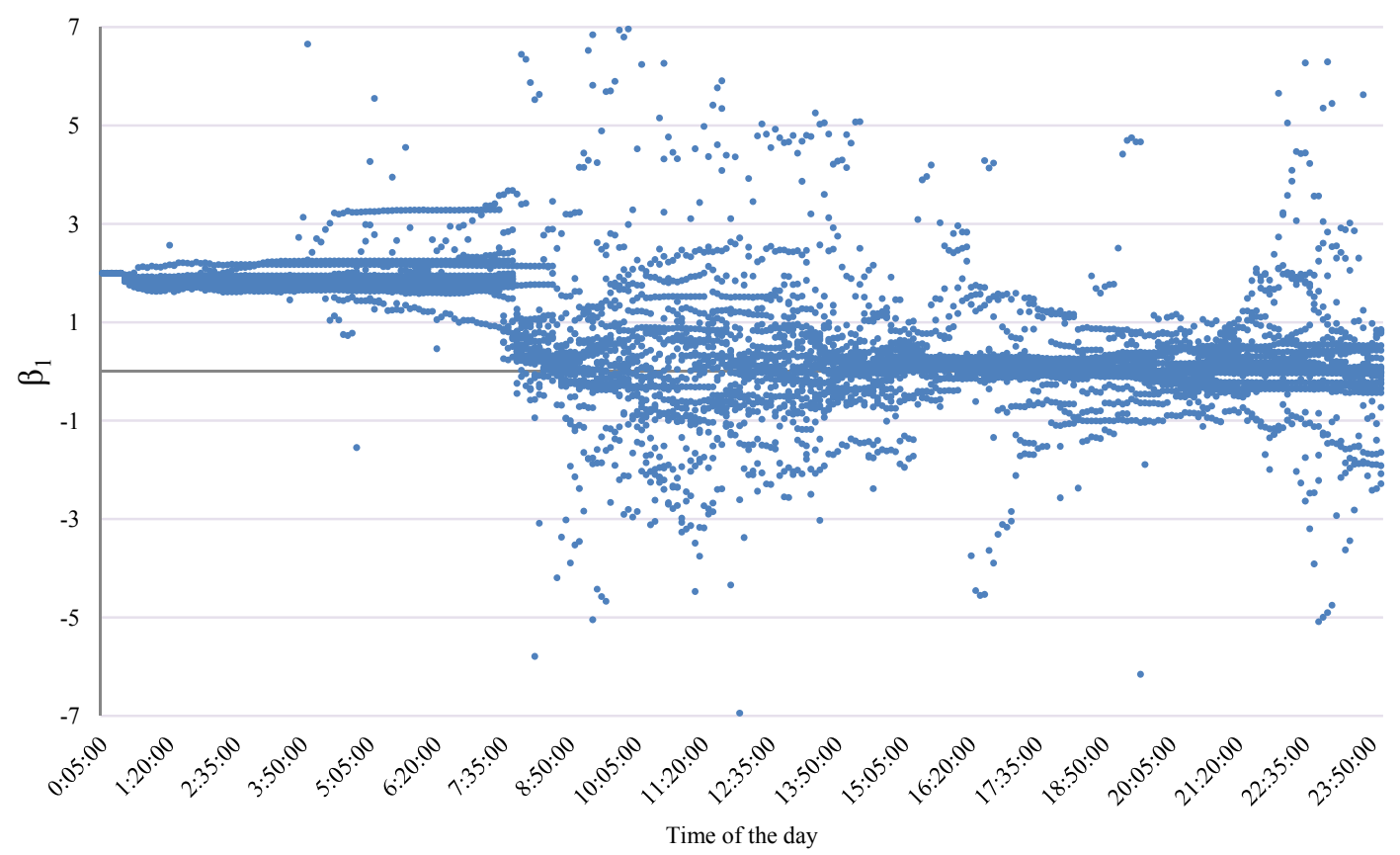

Figure 5.29: Time Varying Values of $\beta_{1}$ Toll 


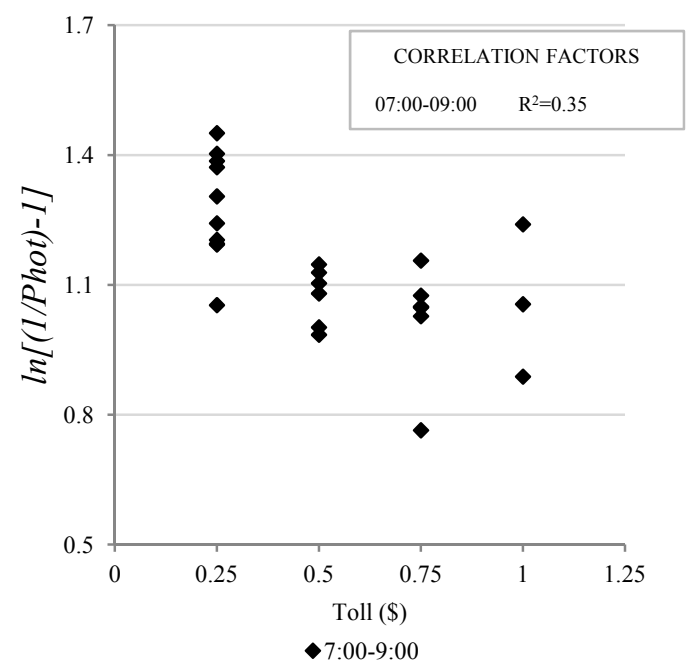

(a) Period I from 07:00 to 09:00

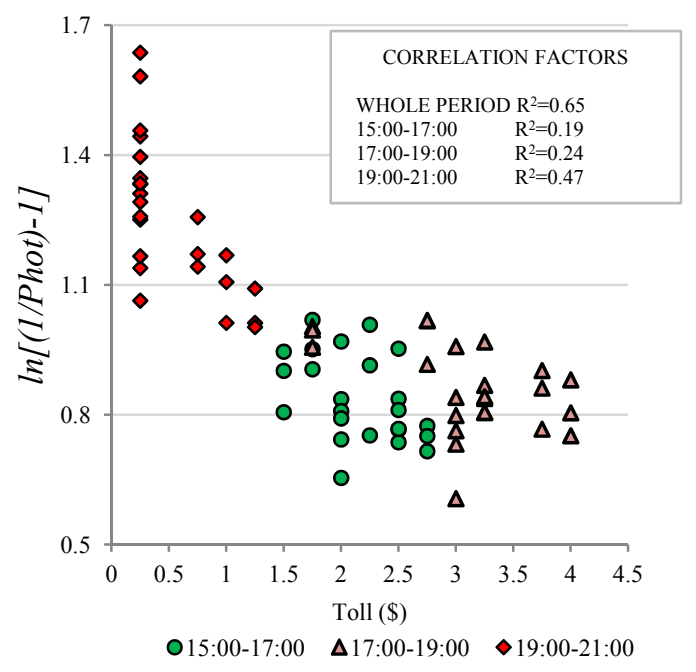

(b) Period II from 15:00 to 21:00

Figure 5.30: Proportion of HOT Users as Function of Toll and Time

In the case of Model 5, which uses the occupancy to explain the variations of the proportion of HOT users, the results are somewhat similar to what was described for the model based on the toll. The estimates obtained for $-\beta_{0}$ are highly variable while the $\beta_{1}$ estimates are symmetrically distributed around zero, indicating that there is no relationship between the observed occupancy and the time varying demand for the HOT lanes. While the observed variability of the bias is a consequence of the occupancy day to day variability and the poor explanatory power of the occupancy, a detailed analysis of the joint variation of the proportion of HOT users and the occupancy was performed to explore why there is no relationship between the occupancy and the HOT demand. Figure 5.33 depicts the variation of the proportion of HOT users and the measured occupancy. In this figure, the data have been clustered depending of the period of the day where they actually happened. In addition, the correlation factors for each period and the dataset as a whole were computed. It is observed that while the correlation factor for the whole group indicates a strong correlation between the variables, the same analysis performed by 
period of day indicates exactly the opposite. This is consistent with the results obtained using the ALMF, that explicitly considers the sequential temporal aspect of the estimation problem.

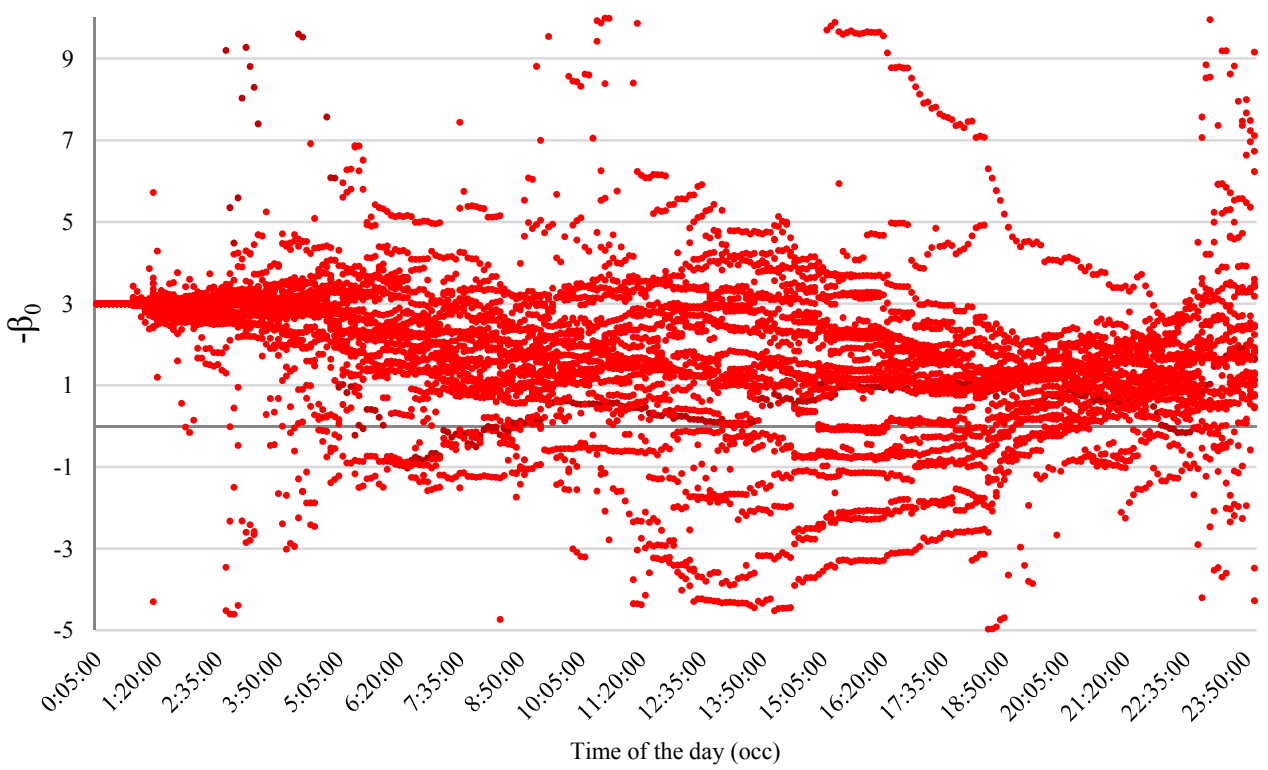

Figure 5.31: Time Varying Values o

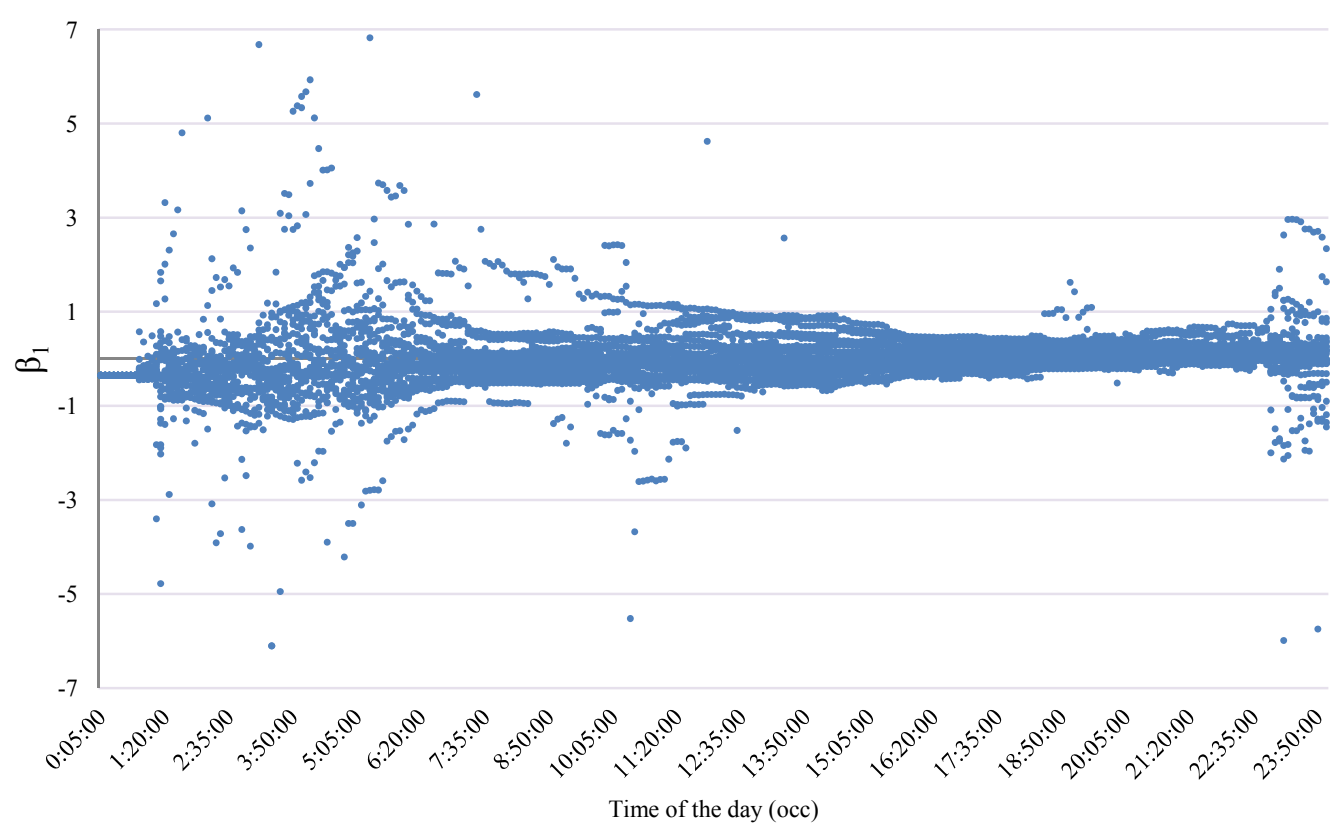

Figure 5.32: Time Varying Values of $\beta_{1}$ Occupancy 


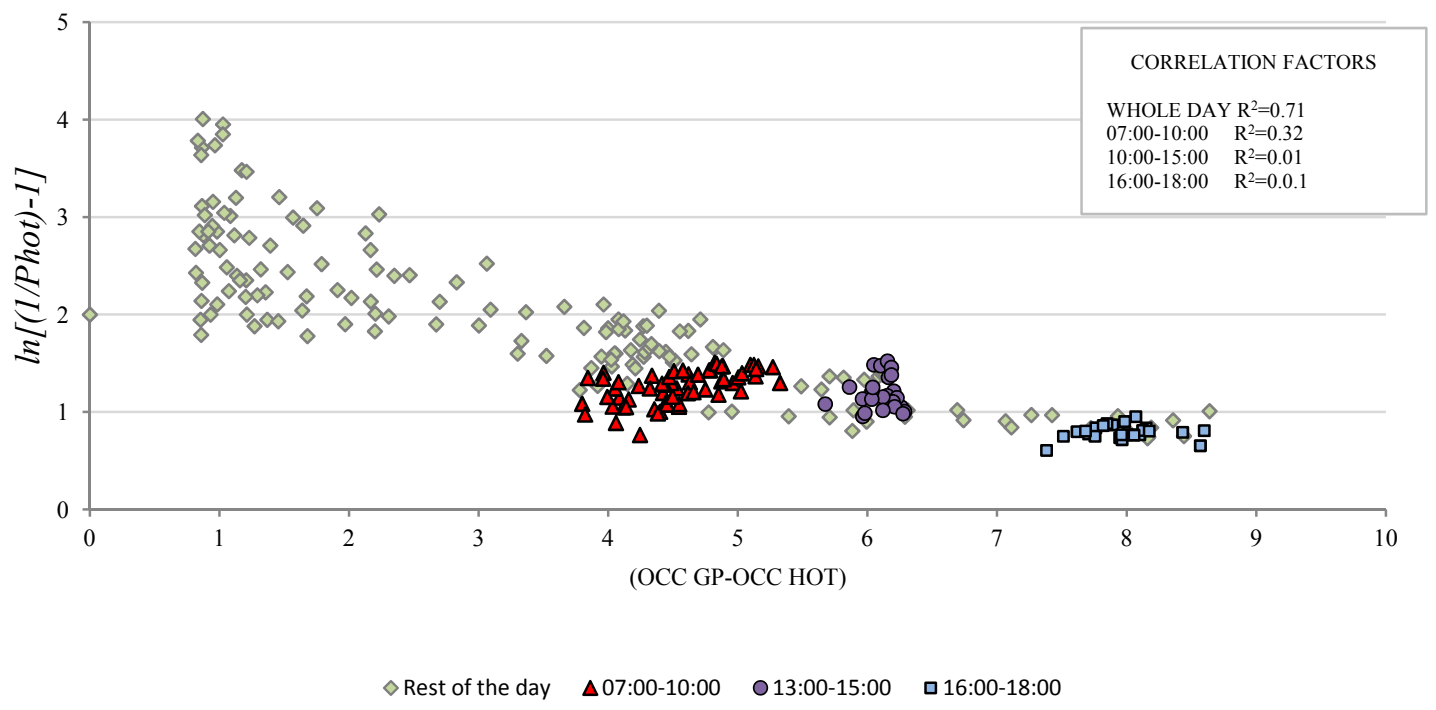

Figure 5.33: Time Varying Values of $\beta_{1}$ Occupancy

In order to assess the significance of the parameters $\beta_{0}$ and $\beta_{1}$, the time varying $95^{\text {th }}$ confidence levels were computed and plotted together with the mean and the standard deviation. Figure 5.34 to Figure 5.41 show the mentioned statistics for all models considered. From the figures it is possible to state that $\beta_{0}$ is significant at $95^{\text {th }}$ confidence level in models 1 to 3 but fails in some period of the day in models 4 and 5. On the other hand, $\beta_{1}$ is significant only in models 1 to 3 for only those periods where the variability in the explanatory variable was observed. When toll is considered simultaneously with the travel time and other reliability metrics such as in models 6 to 8 , the joint estimation produce interesting results. First in the case of model 6 (Figure 5.39), it observed that the parameter associated to the travel time $\left(\beta_{1}\right)$ is significant and relatively constant during the period of interest while during the same period, $\beta_{0}$ experiences significant variations. This is an indication that during congested conditions toll and travel time can be used as explanatory variables, however as suggested by the values of $\beta_{0}$, there are other factors not explicitly considered in this model that increase the attractiveness of the HOT lanes during 
congested conditions. In the case of models 7 and 8 (Figures 5.40 and 5.41 respectively) jointly considering toll and reliability measures produce estimates of $\beta_{0}$ and $\beta_{1}$ that are highly variable during the congested period and thus not suitable to model travelers' behavior in the corridor.
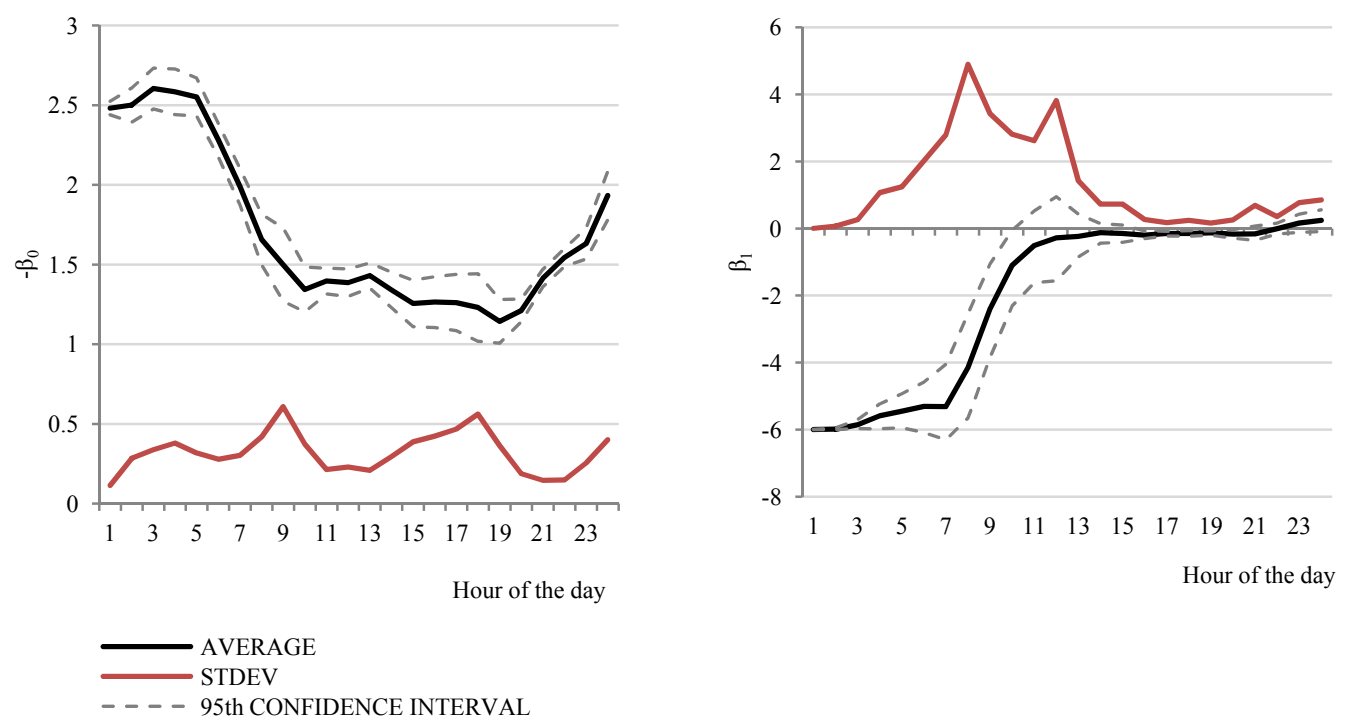

Figure 5.34: $\beta_{0}$ and $\beta_{1}$ Time Varying Mean, $95^{\text {th }}$ Confidence Interval and Standard Deviation with Travel Time as Explanatory Variable
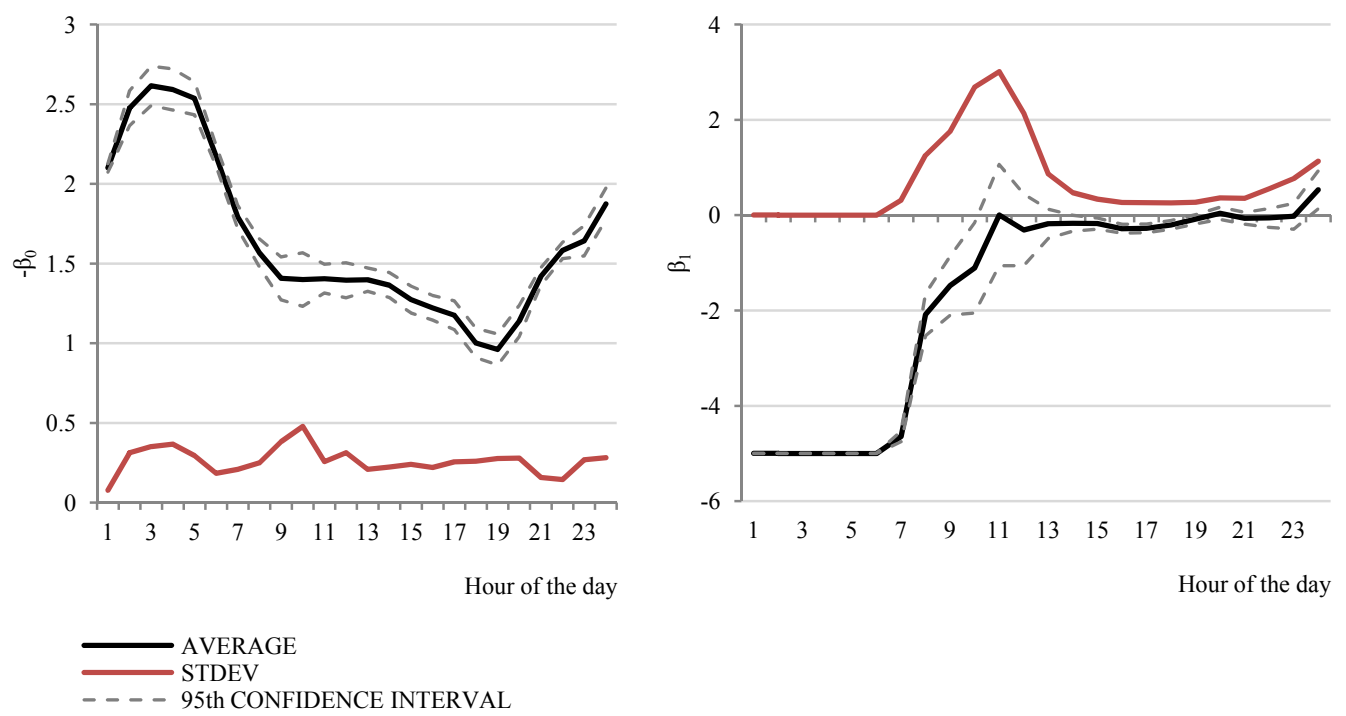

Figure 5.35: $\beta_{0}$ and $\beta_{1}$ Time Varying Mean, $95^{\text {th }}$ Confidence Interval and Standard Deviation with Planning Time Index $95^{\text {th }}$ as Explanatory Variable 

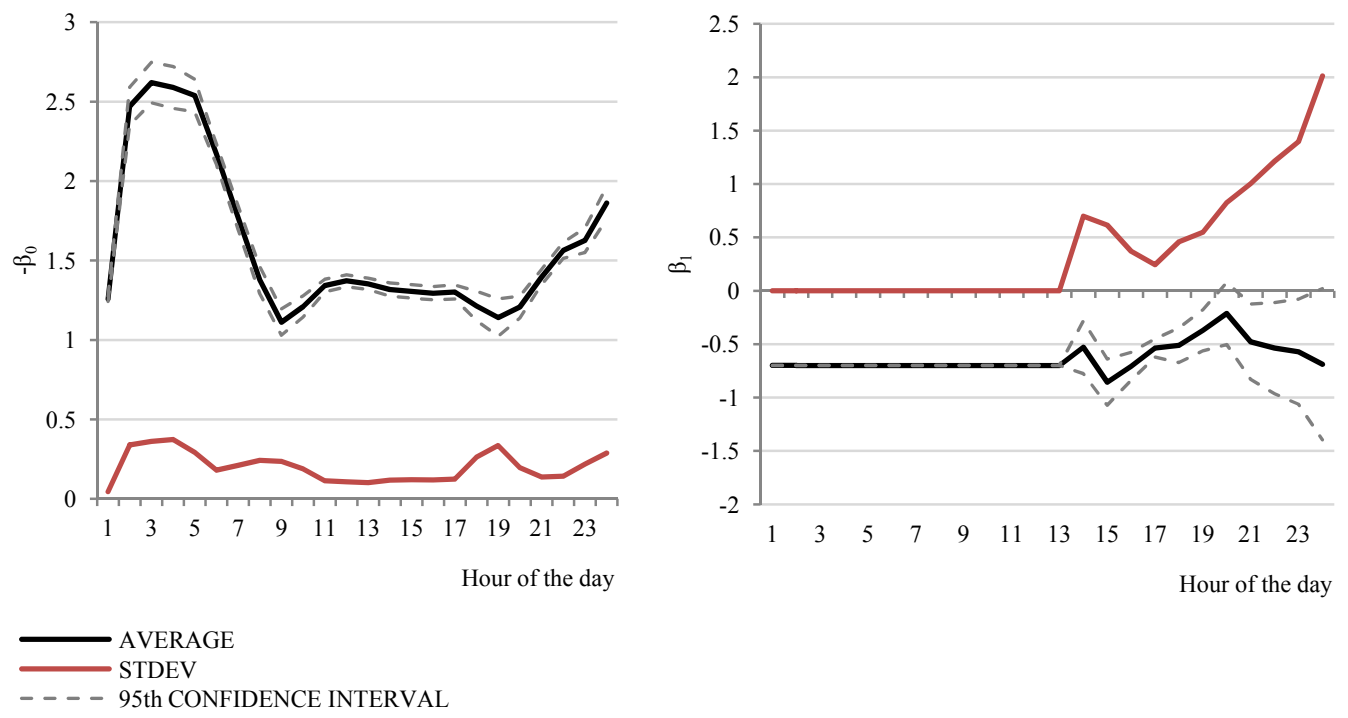

Figure 5.36: $\beta_{0}$ and $\beta_{1}$ Time Varying Mean, $95^{\text {th }}$ Confidence Interval and Standard Deviation with Planning Time Index $\mathbf{8 0}^{\text {th }}$ as Explanatory Variable
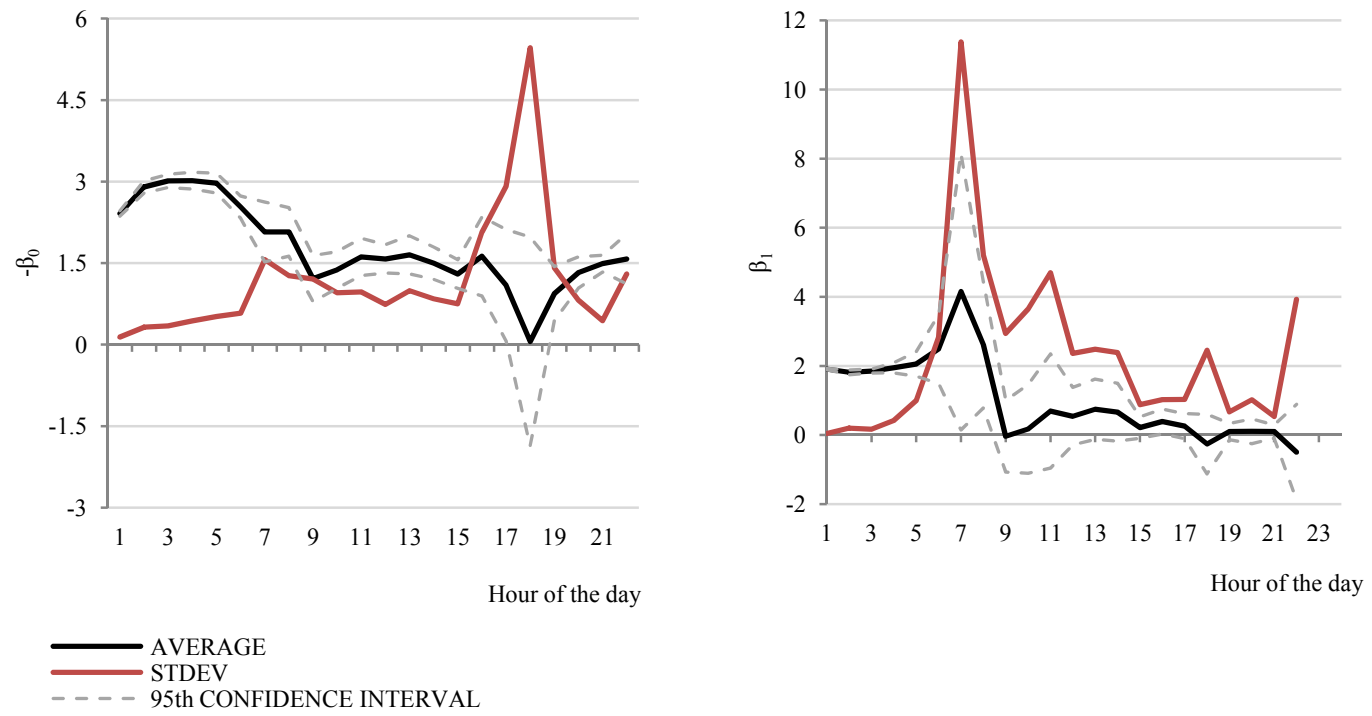

Figure 5.37: $\beta_{0}$ and $\beta_{1}$ Time Varying Mean, $95^{\text {th }}$ Confidence Interval and Standard Deviation with Toll as Explanatory Variable 

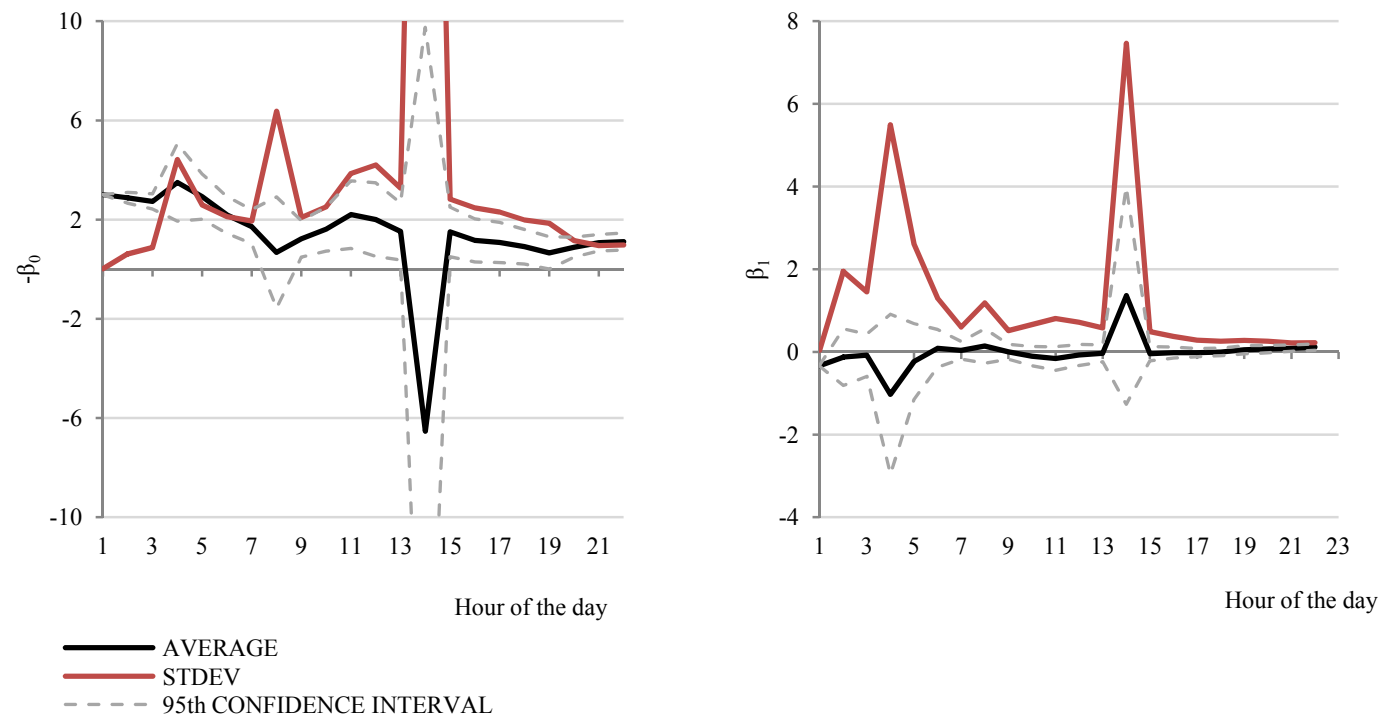

Figure 5.38: $\beta_{0}$ and $\beta_{1}$ Time Varying Mean, $95^{\text {th }}$ Confidence Interval and Standard Deviation with Occupancy as Explanatory Variable
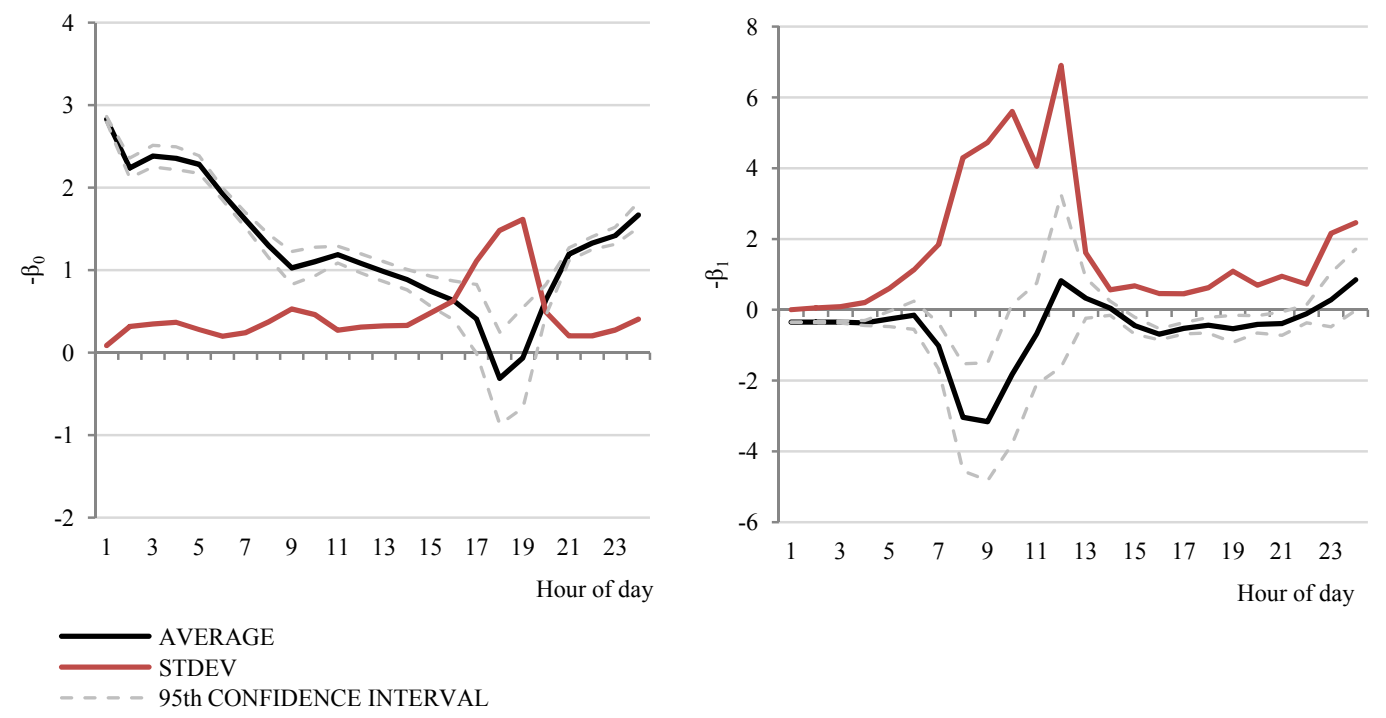

Figure 5.39: $\beta_{0}$ and $\beta_{1}$ Time Varying Mean, $95^{\text {th }}$ Confidence Interval and Standard Deviation with Travel Time and Toll as Explanatory Variables 

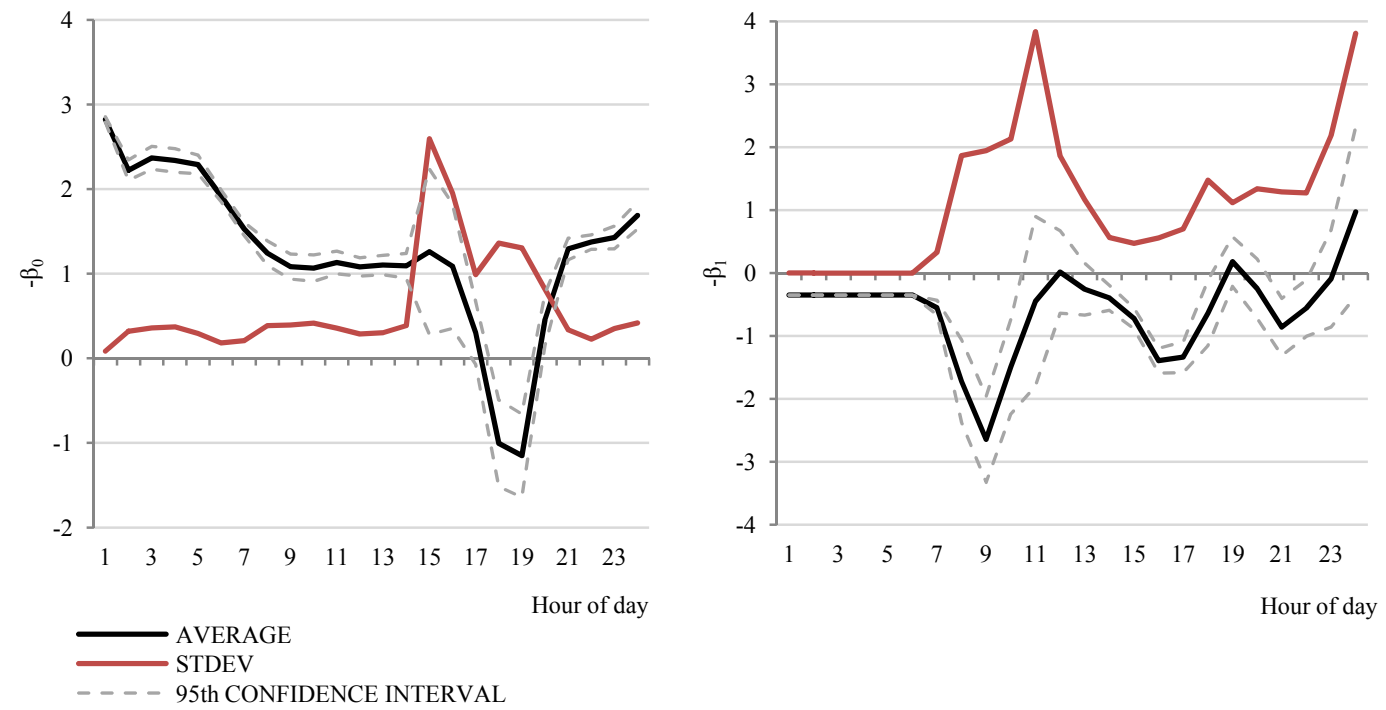

Figure 5.40: $\beta_{0}$ and $\beta_{1}$ Time Varying Mean, $95^{\text {th }}$ Confidence Interval and Standard Deviation with PTI 95 and Toll as Explanatory Variables
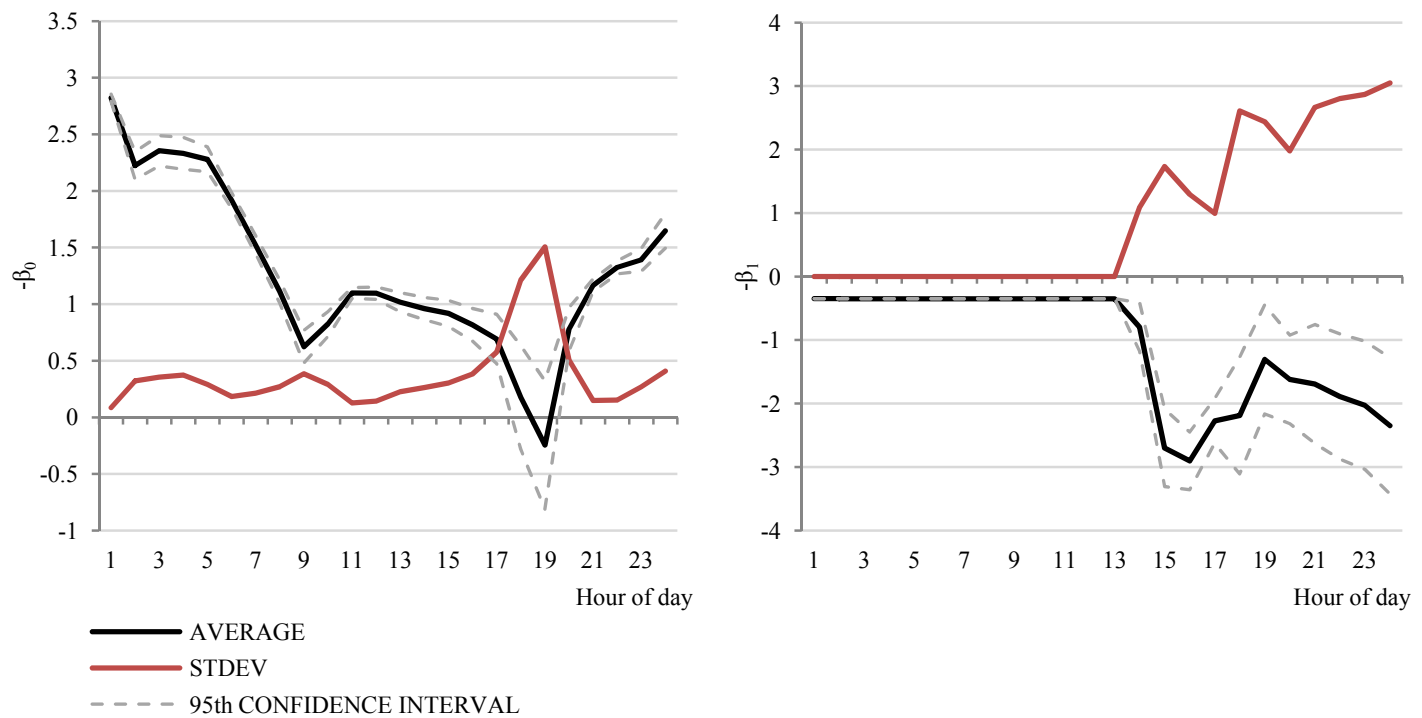

Figure 5.41: $\beta_{0}$ and $\beta_{1}$ Time Varying Mean, $95^{\text {th }}$ Confidence Interval and Standard Deviation with PTI 80 and Toll as Explanatory Variables 
Histograms for the explanatory variables in the models were also plotted for the parameters observed in the PM period from 13:00 to 20:00. The histograms are shown in Figure 5.42 for the travel time, Figure 5.43 for the PTI $95^{\text {th }}$ and Figure 5.44 for the PTI $80^{\text {th }}$ respectively. In all cases, the resulting histograms resemble normal distributions with no biases or over frequent values other than the mean. The latter highlights the random, bounded, and well defined nature of the day-to-day traffic pattern variation.

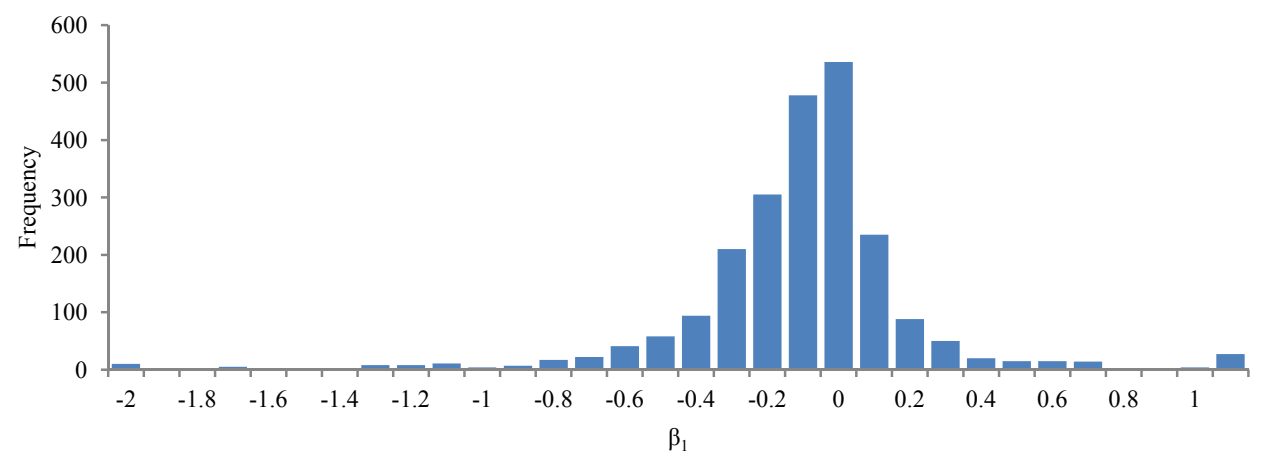

Figure 5.42: $\beta_{1}$ Distribution Between 13:00 and 20:00 when Travel Time is the Explanatory Variable

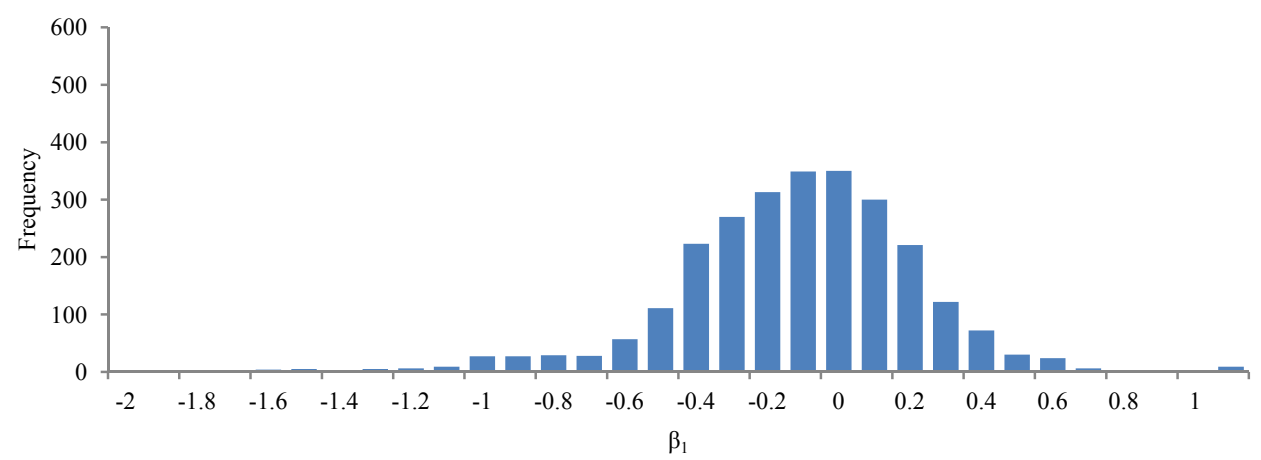

Figure 5.43: $\beta_{1}$ Distribution Between 13:00 and 20:00 when PTI 95 ${ }^{\text {th }}$ is the Explanatory Variable 


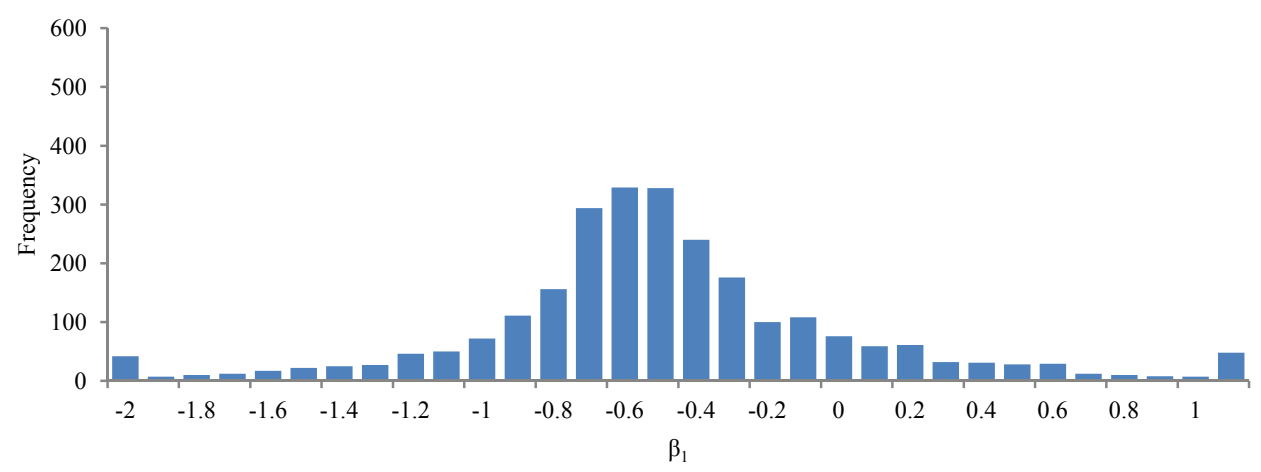

\section{Figure 5.44: $\beta_{1}$ Distribution Between 13:00 and 20:00 when PTI $80^{\text {th }}$ is the Explanatory Variable}

\subsubsection{Sensitivity of the Estimates to the Measured Proportion of HOT Users}

As mentioned in section 5.4.1, the actual number of drivers leaving the GP lanes before reaching the end of the managed system should not be considered as part of the population that actually has to make a decision whether using the HOT or the GP lanes. Nevertheless, there are no off ramps traffic detectors on the section of I-95 where this study takes place. Initially, it was assumed that $10 \%$ of the GP lane traffic at the entrance of the HOT lanes eventually leaves the system by using the off ramps. However the impact of this assumption should be assessed. For this purpose, the proportion of HOT users is computed considering different diversion levels in the GP lanes. Diversion levels are defined as the proportion of the GP counts that afterwards leave the system by using the off ramps. Diversion levels were varied between $10 \%$ and $50 \%$ and the resulting patterns in the proportions of HOT users was compared. Figure 5.45 depicts the proportion of HOT users for various levels of diversion in the GP lanes. It is observed that the effect of changing the GP diversion levels is vertically shifting the position pattern of variation of the proportion of HOT users; however the pattern itself remains invariant. This is an important result in terms that it can be anticipated that assuming 
different diversion levels can only affect the estimation of the bias parameter but not those sensitivities related to the time varying variables of the problem. This conclusion is further supported by Figure 5.46 where the estimation of the parameters $\beta_{0}$ and $\beta_{1}$ has been performed considering $50 \%$ diversion level on the GP counts. In the figure there are two elements that should be highlighted; first the estimated pattern for $\beta_{0}$ is shifted down, reflecting the impact of the assumed diversion in the GP lanes and second, the estimated values of the parameter $\beta_{1}$ closely follow the trend observed for the case where minimal diversion is considered. The latter implies that the effect of different diversion levels do not affect the estimation of the explanatory variable values ( $\beta_{1}$ values), while the bias exhibit a constant shift.

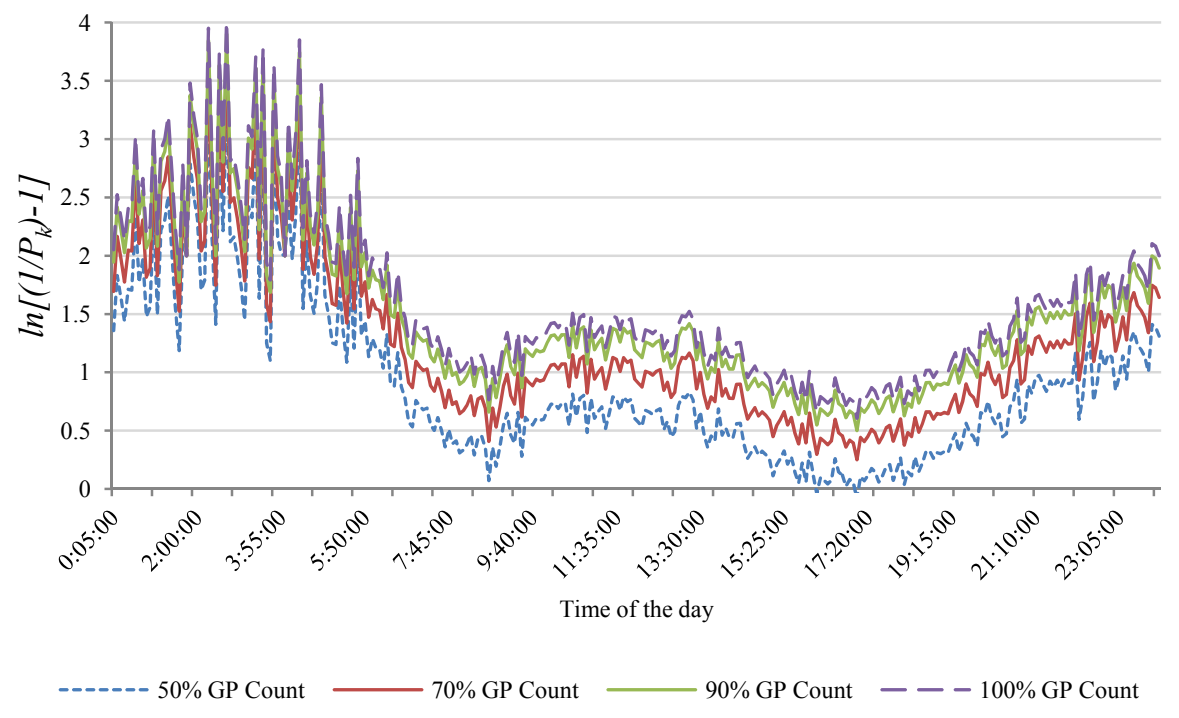

Figure 5.45: $\ln \left[\left(1 / P_{k}\right)-1\right]$ as Function of Time for Different Levels of Diversion in the GPlanes 

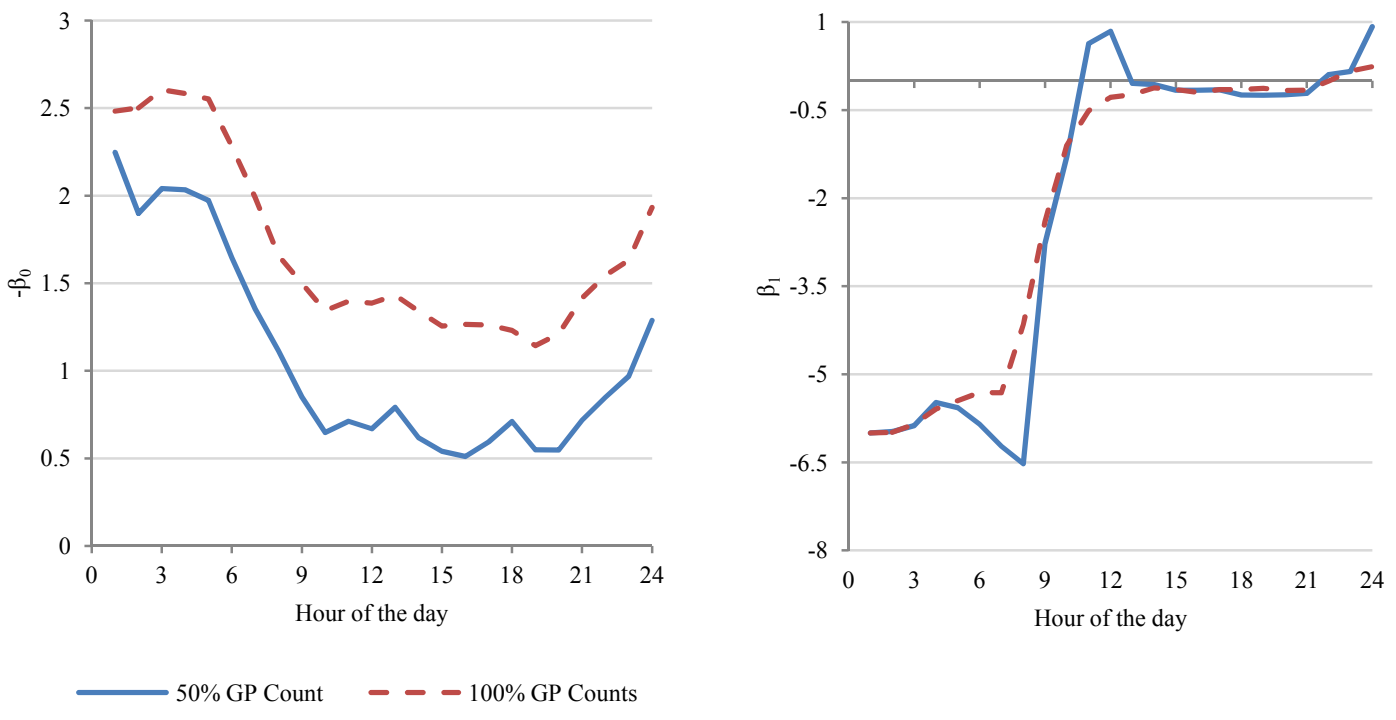

Figure 5.46: $\beta_{0}$ and $\beta_{1}$ Time Varying Mean, $95^{\text {th }}$ Confidence Interval and Standard Deviation with Planning Time Index $95^{\text {th }}$ as Explanatory Variable 


\section{CHAPTER 6}

\section{RESEARCH SUMMARY}

This study has developed a methodology to estimate the user sensitivities to travel time and travel time reliability in a system managed using road pricing strategies. The methodology has focused on the estimation of the user sensitivities to travel time and travel time reliability with special attention to the effect of the time-of the-day and day-to-day traffic variability. The aggregated sensitivities to the travel time and travel time reliability were obtained considering alternative definitions for travel time and travel time reliability. The alternative definitions of the performance measures allowed exploring the mechanisms that best explained the observed the aggregated preferences in the priced system. In the case of travel time, the length of the memory, fading memory rules, and the impact of real time information were explored. Similarly, alternative reliability measures considering different characteristics of the time varying travel time distribution were analyzed. The time varying estimates of the aggregated behavior were obtained by means of applying an adaptive version of the discrete Kalman filter. This chapter summarizes the original contributions of the proposed methodology and conclusions of the study, and discusses the direction of future work.

\subsection{Study Contribution}

Compared to existing traveler behavior estimation methods, the main contributions of this study are the following: 
- The use of detailed ITS data archives allows capturing a detailed description of the aggregated behavior of the priced system users as a function of the time of the day. This allows the identification of the time periods where traveler behavior is more sensitive to travel time, travel time reliability, or none of these measures.

- The use of detailed ITS data archives also allows capturing the typical day to day pattern of variations of the users of the priced system behaviors considering the daily variability of the traffic and environmental conditions, as recorded in the ITS data archives .

- The development of an adaptive discrete filter allows the automatic estimation of the time varying noise statistics of the system, releasing any a priori noise statistic assumption from the estimation process.

- The explicit representation of different learning and processing of travel time information allows capturing specific travel time definitions that best explain the variations of the HOT users for different periods of the day. Such logic for processing travel time information is investigated based on actual aggregated responses to time varying traffic and environmental conditions contained on the ITS data archives.

- The study of different travel time reliability metrics allows identifying a set of metrics, able to capture changes in the travel time distribution as a response to changes in the traffic conditions. The identified metrics were tested in order to ensure that they can consistently represent the changes in the travel time distribution at fine aggregation levels. 


\subsection{Study Conclusions}

In this study, the travel time estimates and traffic counts were obtained from ITS data warehouses. Travel time data is processed using different rules in order to unveil the logic that best fits the observed proportion of the priced facility users and it is also used to estimate various travel time reliability metrics.

Rules regarding the length of the period of time that travelers consider when assessing their experienced travel time are explored. Additionally, the weights that travelers give to old events relative to more recent experiences are investigated. Finally, the impact on system performance assessment of the real time predictive information given to travelers is examined. The results indicate that travelers use their experience in a maximum number of days in their choice processes, and within that period of time, is likely that all the experiences (from all days) receive the same weight. The impact of the real time information revealed to be a function of the time of the day with the PM period the time when this information may be more relevant.

Also, this study investigated the variation of travel time distributions by time of day at fine temporal aggregation levels, the sensitivity of various reliability metrics to these variations, the effect of the aggregation level choices on the calculated reliability metrics, and the amount of data required to estimate stable values of the reliability metrics. The results show that the parameters of travel time distributions vary during the peak period reflecting the effects of the traffic congestion, traffic flow dynamics, and the proportion of the contribution of non-recurrent factors such as incidents to the unreliability of travel time on the investigated facility. 
The results also show that examining combinations of time-variant static distribution parameters and reliability metrics of the GP and HOT lanes can provide valuable information that cannot be obtained when performing the analysis at higher aggregation levels. The $95^{\text {th }}$ Percentile PTI, $80^{\text {th }}$ Percentile PTI, and Misery Index showed continuity and sensitivity in their variations in response to the increase in variability as the congestion in the peak hour. The BI measure was insensitive to the increase in congestion on the GP lane but showed sensitivity to the congestion for the HOT lanes due to the difference in the rates of changes of standard deviations and medians of travel time with the increase of congestion on these facilities, indicating that the interpretation of the results based on this metric should be done with caution. The FOT and Skew Statistics showed inconsistent patterns of travel time variation with the increase in congestion on GP and HOT lanes reflecting the differences in the rates of the increase in the median and standard deviation rates of travel time with the increased congestion on the two facilities and the relative contributions of non-recurrent events to the unreliability.

The results from the study also confirmed that the use of at least 30 minute periods of analysis is preferable to using longer periods for applications that require fine-grained analysis in order to reasonably represent the reliability pattern during congested periods. In addition, the results from the study confirmed that at least one year's worth of data should be collected to obtain a more stable value of reliability metrics. 
Finally, an Adaptive Limited Memory Discrete Kalman Filter (ALMF) algorithm is developed to estimate time varying parameters of the linear system representing the operation of the priced system. The use of the ALMF algorithm allows the estimation of time varying traveler sensitivities to travel time and several travel time reliability metrics at relatively fine aggregation levels. Equally important, the use of the ALMF algorithm allows coping with the problem of estimating the unknown-time varying process noise and measurement noise statistics in a linear system that models the road pricing facility dynamics. The application of the ALMF to a priced corridor in South Florida indicates that there are significant variations in the traveler behavior as a function of the time of the day. However, the results also indicate that the travel time and travel time reliability contributes only marginally to explain the proportion of users of the priced facility. Also the processing of the wealth of data contained in the ITS databases revealed that the day to day variability of the traveler behavior is best represented by means of Gaussian-like probability distributions of the linear system parameters.

\subsection{Future Work}

The following is recommended future work based on this study:

- Even though the developed methodology was developed adapting the discrete Kalman filter to estimate the parameters of a dynamic linear model, it still can be extended to estimate non-linear relationships between the observed preferences for the HOT lanes versus the GP lanes by means of using an extended Kalman filter (EKF) instead. By considering the EKF, the collinear variables in the system can be combined in order to release the constraints imposed by the collinearity. 
- Because the lack of data relative to the off ramps in the system, only an approximation and a sensitivity analysis of the parameter estimates were possible. However FDOT has plans to install detectors in the off ramps in the near future. By incorporating such information, the estimation of the population that is actually deciding choices can be estimated accurately and thus the parameter of the model considered as well.

- Extending the time coverage of the utilized database can eventually provide more details in the long term about the evolution of the system parameters and the travelers' learning of the system performance. In particular, the interest is to capture the period of time required to achieve stable flows on the facility.

- It was assumed during the whole study that travelers can assess the intensity of several performance measures and based on them smoothly trigger decisions. However, there is some evidence that travelers actually leave a small margin for assessing experiences and information; and instead, they tend to repeat their choices as long as the environment remains somewhat familiar to previous successful trials. An alternative decision choice approach framed in a different decision paradigm should be considered to weight the possibility that traffic management actually responds to a different measure of the experiences or to a different decision choice mechanism. 


\section{APPENDICES \\ APPENDIX: ALMF MATLAB CODE}

FUNCTION MY_KALMAN ();

\% KALMAN FILTER IMPLEMENTATION

CLEAR ALL

\% LOAD DATA FROM CSV FILES

OINDEX=CSVREAD ( ' SELECTED_DAYS. CSV' $)$;

Z_HOT=CSVREAD ( 'HOT_PROP. CSV') ;

FÜNCTION OF TIME

OTT_HOT=CSVREAD ( 'TRAV_TIME_HOT . CSV' ) ;

OF TIME

$\because \mathrm{TT}$ GP=CSVREAD ( 'TRAV_TIME_GP.CSV' $)$;

TIME

○FARE=CSVREAD ( ' FARE . CSV' ) ;

○REL_HOT=CSVREAD ( 'RELIAB_HOT.CSV' ) ;

OF TIME

○REL_GP=CSVREAD ( 'RELIAB_GP.CSV' ) ;

TIME

\%OCC_HOT $=$ CSVREAD ( ' OCCUPANCY_HOT.CSV' ) ;

OOCC_GP=CSVREAD ( ' OCCUPANCY_GP.CSV' ) ;

OF TIME

$\%$ [RECORDS, NUM_DAYS ] =SIZE (INDEX' ) ;

THE ANALYSIS

TT_HOT=CSVREAD ( 'OCC_HOT.CSV') ;

TT_GP=CSVREAD ( 'OCC_GP.CSV' $)$;

$\mathrm{LAP}=8$;

\%CONTAINS THE INDEX OF THE SELECTED DAYS FOR ANALYSIS $\because$ CONTAINS THE OBSERVED PROPORTION OF HOT USERS A A

○CONTAINS THE OBSERVED HOT TRAVEL TIME AS FUNCTION ○CONTAINS THE OBSERVED GP TRAVEL TIME AS FUNCTION OF ○CONTAINS THE FARE AS FUNCTION OF TIME $\because$ CONTAINS THE OBSERVED HOT RELIABILITY AS FUNCTION ○CONTAINS THE OBSERVED GP RELIABILITY AS FUNCTION OF \%CONTAINS THE OBSERVED HOT OCCUPANCY AS FUNCTION OF ○CONTAINS THE OBSERVED HOT OCCUPANCY TIME AS FUNCTION ○NUM_DAYS CONTAINS THE NUMBER OF DAYS CONSIDERED IN

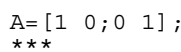

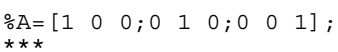

FOR J $=1: 31 \%$ NUM_DAYS

DO ......................

○PERIOD OF TIME CONSIDERED FOR THE NOISE STATISTICS

○STATE TRANSTITION MATRIX (BO;B1)

○STATE TRANSTITION MATRIX (BO;B1;B2)

\%FOR EACH DAY IN THE DATASET

FOR $Z=1: 288$

*** H(Z,:)=[1 (TT_GP $\left.\left.(Z, J)-T T \_H O T(Z, J)\right)\right]$

\%OBSERVATION MAPPING MATRIX B0+B1X1

*** $\% \mathrm{H}(\mathrm{Z},:)=\left[1 \quad\left(T T \_G P(Z, J)-T T \_H O T(Z, J)\right)-F A R E(Z, J)\right]$;

\%OBSERVATION MAPPING MATRIX B0+B1X1+B2X2

$\mathrm{Y}(\mathrm{Z})=\mathrm{Z} \_$HOT $(\mathrm{Z}, \mathrm{J})$;

END

FOR $\quad Z=1: L A P+1$

*** $\mathrm{X}(:, \mathrm{Z})=[3 ;-0.35]$;

*** $\quad \mathrm{X}(:, \mathrm{Z})=[-1.0 ;-0.5 ;-1.0]$;

*** $\quad \mathrm{P}(:,:, \mathrm{Z})=\left[\begin{array}{llll}0.1 & 0.0 ; 0.0 & 0.1\end{array}\right]$;

*** $\quad \% \mathrm{P}(:,:, \mathrm{Z})=\left[\begin{array}{llllllll}0.1 & 0 & 0 ; 0 & 0.1 & 0 ; 0 & 0 & 0.1\end{array}\right]$

*** $Q(:, Z)=[0.0 ; 0.0]$;

$\star * * \quad \% Q(:, Z)=[0.0 ; 0.0 ; 0.0]$

$* * *$

$Q(:,:, Z)=\left[\begin{array}{llll}0.1 & 0 ; 0 & 0.1\end{array}\right]$;

THE STATE NOISE SEQUENCE

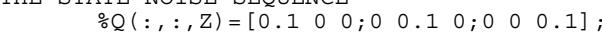

THE STATE NOISE SEQUENCE

$\mathrm{R}(\mathrm{Z})=0.0$;

OBSERVATION NOISE SEQUENCE

END

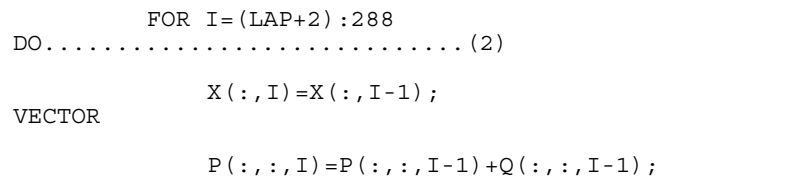

\%OBSERVATION VECTOR

\%THIS IS BECAUSE ESTIMATION STARTSAT LAP+2

○INITIALIZE THE STATE VECTOR (BO;B1)

INITIALIZE THE STATE VECTOR (B0;B1;B2)

\%INITIALIZE THE STATE COVARIANCE (BO;B1)

○INITIALIZE THE STATE COVARIANCE (B0;B1;B2)

INITIALIZE STATE NOISE SAMPLE (B0;B1)

○INITIALIZE STATE NOISE SAMPLE (B0;B1;B2)

\%INITIALIZE TRUE MOMENTS ABOUT THE MEAN OF

○INITIALIZE TRUE MOMENTS ABOUT THE MEAN OF

OINITIALIZE THE OBSERVATION NOISE SAMPLE OINITIALIZE TRUE MOMENTS ABOUT THE MEAN OF THE

$$
\mathrm{R}(\mathrm{I})=\mathrm{Y}(\mathrm{I})-\mathrm{H}(\mathrm{I},:) * \mathrm{X}(:, \mathrm{I}) \text {; }
$$$$
\operatorname{AUX1}=0 \text {; }
$$

AUX1 $=0 ;$
OBSERVATION NOISE SEQUENCE

AUX2 $=0 ;$

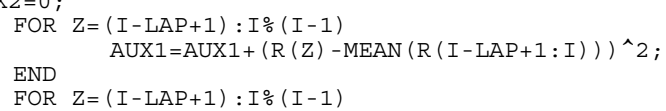

\%EVERY 5 MINUTES

\% (1)A PRIORI ESTIMATE OF THE STATE

\% (2)A PRIORI ESTIMATE OF THE STATE

\% (3) UPDATE OBSERVATION NOISE SAMPLE

\% (4) UPdATE TRUE MOMENTS ABOUT THE MEAN OF THE 


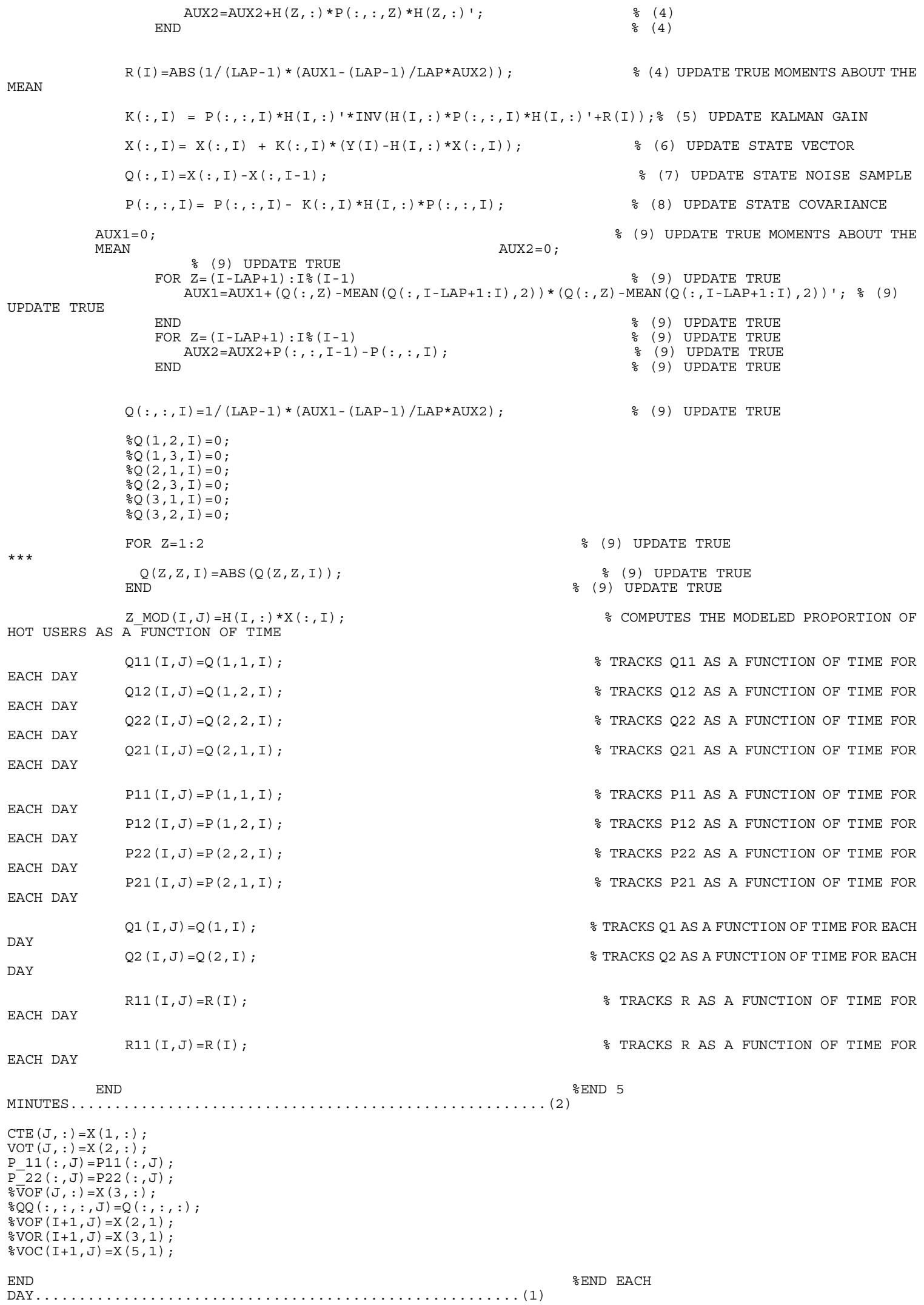

○END EACH 
CSVWRITE ( 'CTE_OUTPUT.CSV', CTE' ') ; CSVWRITE ('VOT_OUTPUT.CSV', VOT') ;

○CSVWRITE ('VOF OUTPUT.CSV', VOF')

CSVWRITE ('HOT MODEL.CSV', Z MOD);

\%CSVWRITE ('VOT OUTPUT. CSV', VOT) ;

OCSVWRITE ('VOF OUTPUT. CSV', VOF) ';

\%CSVWRITE ('VOR OUTPUT.CSV', VOR);

OCSVWRITE ( 'OCC_OUTPUT. CSV ', VOC) ;

$\because$ END

\%END PROGRAM 


\section{APPENDIX B: DATASET I SELECTED DAYS}

\begin{tabular}{|c|}
\hline $5 / 5 / 2010$ \\
\hline $5 / 31 / 2010$ \\
\hline $6 / 1 / 2010$ \\
\hline $6 / 2 / 2010$ \\
\hline $6 / 4 / 2010$ \\
\hline $6 / 28 / 2010$ \\
\hline $7 / 8 / 2010$ \\
\hline $7 / 12 / 2010$ \\
\hline $7 / 15 / 2010$ \\
\hline $7 / 19 / 2010$ \\
\hline $7 / 20 / 2010$ \\
\hline $7 / 21 / 2010$ \\
\hline $8 / 2 / 2010$ \\
\hline $8 / 3 / 2010$ \\
\hline $8 / 16 / 2010$ \\
\hline $8 / 18 / 2010$ \\
\hline $8 / 30 / 2010$ \\
\hline $9 / 13 / 2010$ \\
\hline $9 / 17 / 2010$ \\
\hline $9 / 23 / 2010$ \\
\hline $10 / 7 / 2010$ \\
\hline $10 / 28 / 2010$ \\
\hline $10 / 29 / 2010$ \\
\hline $11 / 4 / 2010$ \\
\hline $11 / 8 / 2010$ \\
\hline $11 / 10 / 2010$ \\
\hline $11 / 15 / 2010$ \\
\hline $11 / 22 / 2010$ \\
\hline $11 / 23 / 2010$ \\
\hline $11 / 25 / 2010$ \\
\hline $11 / 26 / 2010$ \\
\hline
\end{tabular}




\section{REFERENCES}

Abdel-Aty, M., Kitamura, R. and Jovanis, P. (1997), "Using stated preference data for studying the effect of advanced traffic information on drivers' route choice", Transportation Research Part C, Vol. 5, pp. 39-50.

Al-Deek, H., Chandra, S. R., Emam, E. B., \& Klodzinski, J. (2007). "Evaluating effects of toll strategies on route diversion and travel times for origin-destination pairs in a regional transportation network". Transportation Research Record, 2035, 205-215

Alger, S., J. L Dillen, and S. Widlert. 1996. The national Swedish value of time study on the Value of Time, 28-30 October, Wokingham.

Alspach, D. (1974). A Parallel Filter Algorithm for Linear Systems with Unknown Time Varying Statistics, Transactions in Automatic Control, Vol. 19, No. 5, pp. 552-556

Alvarez P., M. Hadi, C. Zhan (2010). "Use of ITS Data to Calibrate Microsospic Simulation Models for Incident Conditions". Transportation Research Record,

Álvarez, Ó., Cantos, P., \& García, L. (2007). "The value of time and transport policies in a parallel road network". Transport Policy, 14(5), 366-376.

Anderson, D. (1989). "Infrastructure pricing policies and the public revenue in African countries”. World Development, 17(4), 525-542.

Ariely, D., Carmon, Z. (2000). Gestalt Characteristics of Experiences: The Defining Features of Summarized Events. Journal of Behavioral Decision Making, 13,pp.191-201

Ariely D., and Zauberman G. (2000). On the Making of an Experience: The Effects of Breaking and Combining Experiences on their Overall Evaluation. Journal of Behavioral Decision Making 13, pp. 219-232.

Armelius, H., \& Hultkrantz, L. (2006). "The politico-economic link between public transport and road pricing: An ex-ante study of the Stockholm road-pricing trial". Transport Policy, 13(1), 162-172.

Armstrong-Wright, A. T. (1986). "Road pricing and user restraint: Opportunities and constraints in developing countries". Transportation Research Part A, 20(2), 123-127.

Arnott, R., De Palma, A., \& Lindsey, R. (1992). "Route choice with heterogeneous drivers and group-specific congestion costs". Regional Science and Urban Economics, 22(1), 71-102. 
Arnott, R., De Palma, A., \& Lindsey, R. (1993). "A structural model of peak congestion: A traffic bottleneck with elastic demand". The American Economic Review, 83(1), $161-179$.

Avineri, E., and Prashker, J. (2006). The Impact of Travel Time Information on Travelers Learning under Uncertainty. Transportation 33, pp. 393-408.

Axhausen, K. W., A. König, G. Abay, J. J. Bates, and M. Bierlaire. 2004. "Swiss value of travel time savings". Proceedings of the European Transport Conference, Strasbourg, France

Bagozzi, R., and Silk, A. (1983). Recall, Recognition and the Measurement of Memory for Print Advertisements. Marketing Science. 2 pp.95-134.

Basso, L. J., \& Zhang, A. (2008). "Sequential peak-load pricing: The case of airports and airlines". The Canadian Journal of Economics. Revue Canadienne d'Economique, 41(3), $1087-1119$.

Beevers, S. D., \& Carslaw, D. C. (2005). "The impact of congestion charging on vehicle emissions in London". Atmospheric Environment, 39(36), 6875-6884

Beckman, M. J, McGuire, C.B., and Winsten, C.B. (1956) Studies in the Economics of Transportation. Yale University Press, New Heaven, CN. (http://www.rand.org/pubs/research_memoranda/2005/RM1488.pdf)

Bekiaris, E., \& Nakanishi, Y. (Eds.) (2004). Economic impacts of intelligent transportation systems: Innovations and case studies. Research in transportation economics, 8. Oxford: Elsevier.32.

Bellei, G., Gentile, G., \& Papola, N. (2002). "Network pricing optimization in multi-user and multimodal context with elastic demand". Transportation Research Part B, 36(9), 779-798.

Ben-Akiva, M. De Palma, A., and Kayasi, I. (1991). Dynamic Network Models and Driver Innformation Systems. Trabsportation Research 25A(5), pp. 251-266.

Bettman, J., (1979). An Information Processing Theory of Consumer Choice. Addison-Wesley, Reading, MA

Bierlaire M., and M. Thémans. (2005). "Development of Swiss models for transportation demand prediction in response to real-time traffic information". Proceedings of the 5th Swiss Transport Research Conference, Ascona.

Boyles S., Voruganti A., Waller T. (2010), Quantifying Travel Time Variability in Transportation Networks. Research Report SWUTC/10/167275-1, Austin, Texas 
Braid, R. M. (1989). "Uniform versus peak-load pricing of a bottleneck with elastic demand". Journal of Urban Economics, 26(3), 320-327

Brownstone, D., \& Small, K. A. (2005). "Valuing time and reliability: Assessing the evidence from road pricing demonstrations". Transportation Research Part A, 39(4), 279-293.

Burris, M. W., \& Pendyala, R. M. (2002). "Discrete choice models of traveller participation in differential time of day pricing programs". Transport Policy, 9(3), $241-451$

Button, K. J., \& Verhoef, E. T. (Eds.) (1998). Road pricing, traffic congestion and the environment:Issues of efficiency and social feasibility. Cheltenham, UK: Edward Elgar

Calfee, J.,\&Winston, C. (1998). “The value of automobile travel time: Implications for congestion policy". Journal of Public Economics, 69(1), 83-102

Cambridge Systematics, Texas A\&M University, Dowling Associates, Street Smarts, H. Levinson and H. Rakha, (2010) Analytical Procedures for Determining the Impacts of Reliability Mitigation http://onlinepubs.trb.org/onlinepubs/shrp2/L03 draft.pdf [accessed on July $29^{\text {th }}$ ]

Cantarella G.E. and Cascetta, E., and (1996). Dynamic Processes and Equilibrium in Transportation Networks: Towards a Unifying Theory. Transportation Science 29(4), pp. 305-329.

Cascetta, E. (1989). A Stochastic Process Approach to the Analysis of Temporal Dynamics in Transportation Networks. Transportation Research 23B, pp. 1-17.

Cascetta, E., and Cantarella G.E. (1991). A Day-to-day and Within-Day Dynamic Stochastic Assignment Model. Transportation Research 25A(5), pp. 277-291.

Carmon, Z., and Kahneman, D. (1996). The Experienced Utility of Queuing: Real Time Affect and Retrospective Evaluations of Simulated Queues, Working paper (http://faculty .insead.edu/Carmon/pdffiles/The\%20Experienced $\% 20$ Utility $\% 200 \mathrm{f} \% 20 \mathrm{Qu}$ euing.pdf), Fuqua School of Business, Duke University, Durham

Chan, K. S., \& Lam, W. H. K. (2005). "Impact of road pricing on road network reliability". Journal of Eastern Asia Society for Transportation Studies, 6, 2060-2075.

Chen, R and H. Mahmassani (2003) Travel Time Perception and Learning Mechanisms in Traffic Networks. Proccedings of the $83^{\text {rd }}$ Transportation Research Board Annual Meeting, Washington, D.C. 
Chin, A. T. H. (1996). "Containing air pollution and traffic congestion: Transport policy and the environment in Singapore". Atmospheric Environment, 30(5), 787-801.

Chui, C. and Chen G.,(1999). Kalman Filtering with Real-Time Applications. Springer-Verlag

Churchill, A., Huber, K., Meldau, E., \& Walters, A. (1972). "Road user charges in Central America". World Bank Staff Occasional Papers, 15. Washington, DC: World Bank.

Courage, K.G. and S. Lee (2008). Development of a Central Data Warehouse for Statewide ITS and Transportation Data in Florida: Phase II Proof of Concept. Florida Department of Transportation.

Dafermos, S. C. (1973). "Toll patterns for multiclass-user transportation networks". Transportation Science, 7, 211-223.

Daganzo, C. F., \& Sheffi, Y. (1977). "On stochastic models of traffic assignment". Transportation Science, 11(3), 253-274.

Dawson, J. A. L., \& Catling, I. (1986). "Electronic road pricing in Hong Kong". Transportation Research Part A, 20(2), 129-134.

De Palma, A., Kilani, M., \& Lindsey, R. (2005). "Congestion pricing on a road network: A study using the dynamic equilibrium simulator METROPOLIS". Transportation Research Part A, 39(7-9), 588-611.

De Palma, A., Lindsey, R., \& Niskanen, E. (2006). "Policy insights from the urban road pricing case studies". Transport Policy, No. 13, Issue 2, pp. 149-161

De Palma, A., Lindsey, R., \& Proost, S. (Eds.) (2007). "Investment and the use of tax and toll revenues in the transport sector". Research in Transportation Economics, 19. Oxford: Elsevier.

De Palma, A., Lindsey, R.,\&Proost, S. (2006). "Research challenges in modeling urban road pricing: An overview". Transport Policy, 13(2), 97-105.

De Palma A., and Papageorgiou, Y. (1989). Heuristic Model of Choice. Northwestern University, Department of Civil Engineering

DeCorla-Souza, P. (2002). "Estimating benefits from mileage-based vehicle insurance, taxes, and fees". Transportation Research Record, 1812, 171-178.

DeCorla-Souza, P. (2004). "Recent U.S. experience: Pilot projects". In G. Santos (Ed.), Road pricing: Theory and evidence, research in transportation economics (Vol. 9, pp. 283-308). Oxford: Elsevier 
DeCorla-Souza, P. (2006). "FAST Miles: Benefits and financial feasibility of multimodal investment and pricing strategy". Transportation Research Record, 1960, 152-158.

DeSerpa, A. (1971). "A Theory of the Economics of Time". The Economic Journal, No.81, pp. 828-846.

Dimitriou, L., \& Tsekeris, T. (2009). "Evolutionary game-theoretic model for dynamic congestion pricing inmulti-class traffic networks". NETNOMICS: Economic Research and Electronic Networking Economics (Vol. 9). Oxford: Elsevier

Einhorn H., and Hogarth R. (1981). Behavioral Decision Theory: Processes of Judgment and Choice. Annals of Psychology 32, pp. 53-88.

Eliasson, J., \& Mattsson, L.-G. (2006). "Equity effects of congestion pricing: Quantitative methodology and a case study for Stockholm". Transportation Research Part A, 40(7), 602-620.

Erhardt, G. D., Koppelman, F. S., Freedman, J., Davidson, W. A., \& Mullins, A. (2003). "Modeling the choice to use toll and high-occupancy vehicle facilities". Transportation Research Record, 1854, 135-143

FDOT Florida Statewide Operations Performance Measures and Data Collection, Florida Department of Transportation Traffic Engineering and Operations Office, (2008). http://www.floridaits.com/PDFs/PerfMeas/081028-ITS_PM_Annual_Report_2008\%20-fi nal.pdf [accessed on July $29^{\text {th }}$ ]

FHWA Federal Highway Administration, (2004) Archived Data Management Systems - A Cross-Cutting Study. Publication FHWA-JPO-05-044. FHWA, U.S. Department of Transportation.

Franklin, J. P. (2006). "The equity effects of roadway tolls: An application of Hicksian welfare measures with income effects". In Proceedings of the 11th international conference on travel behaviour research, Kyoto Japan

Franklin, J. P. (2008). "Nonparametric distributional analysis of a transportation policy: Stockholm's congestion pricing trial". In Proceedings of the transportation research board $87^{\text {th }}$ annual meeting. Washington, DC.

Friesz, T., Bernstein D., Mehta, N., Tobin, R., and Ganjalizadeh, S., (1994). Day-to-day Dynamic Network Disequilibria and Idealized Traveler Information Systems. Operations Research 42(6), pp. 1120-1136.

Gakenheimer, R. (1999). "Urban mobility in the developing world". Transportation Research Part A, 33(7-8), 671-689 
Gibbons, E., \& O'Mahony, M. (2002). "External cost internalisation of urban transport: A case study of Dublin”. Journal of Environmental Management, 64(4), 401-410.

GDOT Georgia Department of Transportation, (2011) Strategic Planning. http://www.dot.state.ga.us/informationcenter/programs/Documents/Strategic/FY2011-Str ategicPlan-FINAL.pdf $\left[\right.$ accessed on July $\left.29^{\text {th }}\right]$

Gelb, A.,(1989) Applied Optimal Estimation, The MIT Press, Massachusetts Institute of Technology, Cambridge, MA.

Golob, T. F. (2001). "Joint models of attitudes and behavior in evaluation of the San Diego I-15 congestion pricing project”. Transportation Research Part A, 35(6), 495-514.

Goodwin, P. (2004). "Congestion charging in Central London: Lessons learned". Planning Theory \& Practice, 5(4), 501-505.

Gronau, R. (1976). "Economic Approach to Value of Time and Transportation Choice". Transportation Research Record, No.587, pp.1-5

Gulipalli, P. K. Credit-Based Congestion Pricing: Impact Assessment for the Dallas-Fort Worth,Region and Policy Recommendations. Master's Thesis, The University of Texas at Austin, 2005.

Gunn, H. and C. Rohr. (1996). "Research into the value of travel time savings and losses". Paper presented at the PTRC International Conference on the Value of Time, 28-30 October, Wokingham

Harrison, W. J., Pell, C., Jones, P. M., \& Ashton, H. (1986). "Some advances in model design developed for the practical assessment of road pricing in Hong Kong". Transportation Research Part A, 20(2), 135-143.

Hau, T. D. (1990). "Electronic road pricing: Developments in Hong Kong 1983-89". Journal of Transport Economics and Policy, 24(2), 203-214.

Heiner, R., (1983). The Origin of Predictable Behavior. American Economic Review, 73 , pp. $560-595$

Helbing, D., Schonhof, M., and Kern D. (2002). Volatile Decision Dynamics: Experiments, Stochastic Description, Intermittency Control, and Traffic Optimization. New Journal of Physics 4, pp. 33.1-33.16.

Hensher, D. A., \& Puckett, S. M. (2008). "Assessing the influence of distance-based charges on freight transporters". Transport Reviews, 28(1), 1-19. 
Hess, S., Smith, C., Falzarano, S., \& Stubits, J. (2008). "Measuring the effects of different experimental designs and survey administration methods using an Atlanta Managed Lanes stated preference survey". In Proceedings of the transportation research board 87th annual meeting. Washington, DC.

HCM (2010) Highway Capacity Manual. TRB, National Research Council, Washington, D.C.

Hensher, D. (1976) "Review of Studies Leading to Existing Values of Travel Time". Transportation Research Record, No. 587, pp. 30-41.

Hilborn C. and D. Lainiotis (1969). Optimal Estimation in the Presence of Unknown Parameters, IEEE Transactions Systems Science and Cibernetics, Vol. 5, Issue 1, pp. $38-42$

Hills, P. (1984). "Hong Kong's roads: Clearing the chaos". Cities (London, England), 1(3), 234-242.

Hogarth R. (1987). Judgment and Choice the Psychology of Decision $2^{\text {nd }}$ Edition. John Wiley and Sons, New York.

Holguín-Veras, J. (2008). "Necessary conditions for off-hour deliveries and the effectiveness of urban freight road pricing and alternative financial policies in competitive markets". Transportation Research Part A, 42(2), 392-413.

Holguín-Veras, J., Sackey, D., Hussain, S., \& Ochieng, V. (2003). "Economic and financial feasibility of truck toll lanes". Transportation Research Record, 1833, 66-72.

Horowitz, J.L. (1984). The Stability of Stochastic Equilibrium in a Two-Link Transportation Network. Transportation Research 18B(1), pp. 13-28.

Iida, Y., Akiyama, T., and Uchida T. (1992). Experimental Analysis of Dynamic Route Choice Behavior . Transportation Research 26B(1), pp. 17-32.

Ison, S. (1998). "A concept in the right place at the wrong time: Congestion metering in the city of Cambridge". Transport Policy, 5(3), 139-146. doi

Jackson,W. and Jucker, J. (1981), “An empirical study of travel time variability and travel choice behavior.", Transportation Science, Vol. 16, pp. 460-475.

Jazwinski, A. (2007). Stochastic Processes and Filtering Theory, Academic Press, New York

Jensen-Butler, C., Sloth, B., Larsen, M. M.,Madsen, B., \& Nielsen, O. A. (Eds.) (2008). Road pricing, the economy and the environment. Berlin-Heidelberg: Springer. 
Jha, M., Madanat S., and Peeta, S. (1998). Perception Updating and Day-to-Day Travel Choice Dynamics in Traffic Networks with Information Provision. Transportation Research 6C(3), pp. 189-212.

Johansen, F. (1989). "Toll road characteristics and toll road experience in selected South East Asia countries". Transportation Research Part A, 23(6), 463-466.

Johnson, M. (1966). “ Travel Time and the Price of Leisure”. Western Economic Journal, No. 4, pp. 135-145.

Joumard, R., Lamure, C., Lambert, J., \& Tripiana, F. (1996). "Air quality and urban space management”. The Science of the Total Environment, 189-190, 57-67.

Jovicic, G., \& Hansen, C. O. (2003). "A passenger travel demand model for Copenhagen".Transportation Research Part A, 37(4), 333-349.

Julier, S.,Uhlmann, J. and Durrant-White, H.(1995). A new approach for filtering nonlinear systems. In Proceedings of the American Control Conference, pp. 1628-1632

Kahneman, D., Frederickson, B., Schreiber, C., and Redelmeier, D. (1993). When More Pain is preferred to Less: Adding a Better End. Psychological Science, 4, pp.401-405.

Kalman, R.E.(1960). A new approach to linear filtering and prediction problems. Journal of Basic Engineering (ASME), 82D, pp. 35-45

Kaysi, I. (1991). Framework and Models for Provision of Driver Information System, $\mathrm{PhD}$. Thesis, Department of Civil Engineering, Massachusetts Institute of Technology, Cambridge, MA

Khazzoom, D. J. (1991). "The impact of a gasoline tax on auto exhaust emissions". Journal of Policy Analysis and Management, 10(3), 434-454.

Kim, K. S.,\&Hwang, K. (2005). "An application of road pricing schemes to urban expressways in Seoul”. Cities (London, England), 22(1), 43-53.

Knickerbocker, B. (2000). "Forget Crime - But Please Fix the Traffic." Christian Science Monitor.

Kockelman, K.M.,\&Kalmanje, S. (2005). "Credit-based congestion pricing:Apolicy proposal and the public's response". Transportation Research Part A, 39(7-9), 671-690.

Kunchornrat, J., Pairintra, R., \& Namprakai, P. (2008). "Sustainable energy management in urban transport: The public's response of road congestion pricing in Thailand". Renewable and Sustainable Energy Reviews, 12(8), 2211-2226. 
Kuwahara, M. (2007). "A theory and implications on dynamic marginal cost". Transportation Research Part A, 41(7), 627-643.

Laih, C.-H. (1994). "Queueing at a bottleneck with single- and multi-step tolls". Transportation Research Part A, 28(3), 197-208.

Lam, T. (2004). "Evaluating value-pricing projects with both scheduling and route choices". Regional Science and Urban Economics, 34(2), 225-240.

Larsen, O. I., \& Ostmoe, K. (2001). "The experience of urban toll cordons in Norway. Lessons for the future". Journal of Transport Economics and Policy, 35(3), 457-471.

Lawphongpanich, S., Hearn, D. W., \& Smith, M. J. (Eds.) (2006). Mathematical and computational models for congestion charging. New York: Springer

Leape, J. (2006). "The London congestion charge". The Journal of Economic Perspectives, 20(4), 157-176.

Lee, K.-S., \& Hobeika, A. G. (2007). “Application of dynamic value pricing through enhancements to TRANSIMS". Transportation Research Record, 2003, 7-16.

Levinson, D. (2002). Financing transportation networks. Cheltenham, UK: Edward Elgar

Levinson, D., \& Rafferty, P. (2004). "Delayer pays principle: Examining congestion pricing with compensation". International Journal of Transport Economics, 31(3), 295-311.

Li, H. (2004). "Investigating Morning Commute Route Choice Behavior Using Global Positioning Systems and Multi-day Travel Data", PhD thesis, Georgia Institute of Technology (USA).

Li, H., Bliemer, M. C. J., \& Bovy, P. H. L. (2007). Optimal toll design from reliability perspective. In Proceedings of the 6th triennial symposium on transportation analysis. Phuket, Thailand.

Li, H., Guensler, R. and Ogle, J. (2005), "Analysis of morning commute route choice patterns using global positioning system-based vehicle activity data", Transportation Research Record: Journal of the Transportation Research Board, Vol. 1926, pp. 162-170.

Li, R., Rose, G., Sarvi M. (2006), Using Automatic Vehicle Identification data to gain insight into travel time variability and its causes. $85^{\text {th }}$ Annual Meeting of the Transportation Research Board, Washington D.C.

Lim, C. C. (1997). "The status of transportation demand management in Greater Vancouver and energy implications". Energy Policy, 25(14-15), 1193-1202. 
Lindsey, R. (2005). "Recent developments and current policy issues in road pricing in the US and Canada". European Transport, 31, 46-66.

Link, H. (2008). "Acceptability of the German charging scheme for heavy goods vehicles: Empirical evidence from a freight company survey". Transport Reviews, 28(2), 141-158.

Litman T. (2011) Changing Vehicle Travel Price Sensitivities, The Rebounding Rebound Effect, Victoria Transport Policy Institute

Lomax T., D. Schrank, S. Turner, and R. Margiotta, (2003) Report for Selecting Travel Reliability Measures, http://tti.tamu.edu/documents/474360-1.pdf [accessed on July $29^{\text {th }}$ ]

Lu, X., Gao, S., and Ben-Elia, E. (2010). Information Impacts on Route Choice and Learning Behavior in a Congested Networkl: An Experimental Approach. Presented at the Transportation Research Board $90^{\text {th }}$ Annual Meeting, Washington D.C.

Luo, Q., Juan, Z., Sun, B., \& Jia, H. (2007). "Method research on measuring the external costs of urban traffic congestion". Journal of Transportation Systems. Engineering and Information Technology, 7(5), 9-12.

Mahmassani H.S., and Chang G-L., Herman, R. (1986). Individual Decision and Collective Effects in a Simulated Traffic System. Transportation Science 20(4), pp. 258-271

Mahmassani H.S., and Chang G-L. (1986). Experiments with Departure Time Choice Dynamics of Urban Commuters. Transportation Research 20B(4), pp. 297-320

Mahmassani H.S. (1990) Dynamic Models of Commuter Behavior: Experimental Investigation and Application to the Analysis of Planned Traffic Disruptions. Trabsportation Research 24A(6), pp. 465-48

Markose, S., Alentorn, A., Koesrindartoto, D., Allen, P., Blythe, P., \& Grosso, S. (2007). "A smart market for passenger road transport (SMPRT) congestion: An application of computational mechanism design". Journal of Economic Dynamics \& Control, 31(6), 2001-2032.

Matsuda,W., Tsukada, Y.,\&Kikuchi,M. (2005). "Flexible charge measures used on toll roads: An analysis of demonstration projects in Japan". Transportation Research Record, 1932, 137-146.

Mattsson, L.-G. (2008). "Road pricing: Consequences for traffic, congestion and location”. In C. Jensen-Butler, B. Sloth, M. M. Larsen, B. Madsen,\&O. A. Nielsen (Eds.), Road pricing, the economy and the environment (pp. 29-48). Berlin-Heidelberg: Springer. 
Mayet, J., \& Hansen, M. (1999). "Congestion pricing with continuously distributed values of time". Journal of Transport Economics and Policy, 34(3), 359-369

Mcdonald, J. F. (2004). "Road pricing in practice and theory". The Review of Network Economics, 3(4), 347-355.

Mitchell, G. (2005). "Forecasting environmental equity: Air quality responses to road user charging in Leeds, UK". Journal of Environmental Management, 77(3), 212-226.

Mehra, R. (1972). Approaches to Adaptive Filtering, IEEE Transactions on Automatic Control, Vol. 17, No. X, pp. 693-698

Morrison, S. A. (1986). "A survey of road pricing". Transportation Research Part A, 20(2), pp. 87-97.

Mun, S.-I. (1999). "Peak-load pricing of a bottleneck with traffic jam". Journal of UrbanEconomics, 46(3), 323-349

Murray, P. M., Mahmassani, H. S., \& Abdelghany, K. F. (2001). "Methodology for assessing High-Occupancy Toll-lane usage and network performance". Transportation Research Record,1765, 8-15.

Myers K. and B. Tapley (1976). Adaptive Sequential Estimation with Unknown Noise Statistics, IEEE Transactions in Automatic Control, Vol. 21, No. 4, pp. 520-523

Newbery, D. M. (1990). "Pricing and congestion: Economic principles relevant to pricing roads". Oxford Review of Economic Policy, 6(2), 22-38.

Newell, A., and Simon, H. (1972) Human Problem Solving. Prentice-Hall, Englewood Cliffs, NJ.

Nielsen, O. A. (2004). "Behavioral responses to road pricing schemes: Description of the Danish AKTA experiment”. Journal of Intelligent Transportation Systems, 8(4), 233-251.

Noland, R. B., Quddus, M. A., \& Ochieng, W. Y. (2008). "The effect of the London congestion charge on road casualties: An intervention analysis". Transportation, 35(1), 73-91.

National Transportation Operation Coalition (NOTC), (2005) Performance Measurement Initiative, Final Report. http://downloads.transportation.org/2005am/bod/A\%20Few\%20Good $\% 200 p s \% 20 \mathrm{PM} \%$ 20Report.pdf [accessed on July $29^{\text {th }}$ ]

NIST/SEMATECH (2011) e-Handbook of Statistical Methods, http://www.itl.nist.gov/div898/handbook/, [accessed on July $29^{\text {th }}$ ] 
Odeck, J., \& Brathen, S. (2002). "Toll financing in Norway: The success, the failures and perspectives for the future". Transport Policy, 9(3), 253-260.

Oh, J. E., Labi, S., \& Sinha, K. C. (2007). "Implementation and evaluation of self-financing highway pricing schemes: A case study". Transportation Research Record, $1996,25-33$.

Otterström, T. (1995). "Pricing of environmental impacts of emissions from road traffic - recent and present research in Finland". The Science of the Total Environment, 169(1-3), 311-319.

Parry, I. W. H., Walls, M., \& Harrington, W. (2007). "Automobile externalities and policies”. Journal of Economic Literature, 45(1), 373-399.

Peeta, S., \& Ziliaskopoulos, A. K. (2001). "Foundations of dynamic traffic assignment: The past, the present and the future". Networks and Spatial Economics, 1(3-4), 233-265.

Peeta S., and Pasupathy R. (2001). Analyzing Traffic System Evolution Using Multi-Agent Simulation. Presented at the Transportation Research Board $80^{\text {th }}$ Annual Meeting, Washington D.C.

Pickford, A. T. W., \& Blythe, P. T. (2006). "Road user charging and electronic toll collection". London: Artech House

Pretty, R. L. (1988). "Road pricing: A solution for Hong Kong?". Transportation Research Part A, 22(5), 319-327.

Proost, S., \& Van Dender, K. (2001). "The welfare impacts of alternative policies to address atmospheric pollution in urban road transport". Regional Science andUrban Economics, 31(4), 383-411.

Proost, S., Van Dender, K., Courcelle, C., De Borger, B., Peirson, J., Sharp, D., et al. (2002). "How large is the gap between present and efficient transport prices in Europe?" Transport Policy, 9(1), 41-57.

Pu, W., (2010), Analytic Relationships between Travel Time Reliability Measures. $90^{\text {th }}$ Annual Meeting of the Transportation Research Board, Washington D.C.

$\mathrm{Pu}$ W., A. Messe, J. Andrew and L. Long, (2010), Exploring Travel Time Reliability under Different Circumstances: A Case Study. $90^{\text {th }}$ Annual Meeting of the Transportation Research Board, Washington D.C.

Rakha H., I. El-Shawarby, and M. Arafeh, (2007) Trip Travel Time Reliability: Issues and Solutions. Intelligent Transportation Systems Conference, ITSC. 
Ramjerdi, F., L. Rand, and K. Saelensminde. (1997). "The Norwegian value of time study: Some preliminary results". Oslo: Institute of Transport Economics.

Raol J., G. Girija and J. Singh, (2004) Modelling and Parameter Estimation of Dynamic Systems, The Institution of Electrical Engineers, London 2004

Reichman, S. (1976). "Conceptual Problems in Evaluation of Travel Time", Transportation Research Record, pp. 24-29.

Richards, M. G. (2006). Congestion charging in London. The policy and the politics. Basingstoke, UK: Palgrave Macmillan

Roth, G. (1996). "Roads in a market economy”. Aldershot, UK: Ashgate Publishing.

Roth, G. (ed.) (2006). "Street smart: Competition, entrepreneurship, and the future of roads". New Brunswick, NJ: Transaction Publisher.

Santos, G., \& Fraser, G. (2006). "Road pricing: Lessons from London”. Economic Policy, 21(46), 264-310.

Santos, G., Newbery, D., \& Rojey, L. (2001). "Static vs. demand sensitive models and the estimation of efficient tolls: An exercise for eight Englist towns". Transportation Research Record, 1747, 44-50.

Saurí, S., Argote, J., Salas, M., \& Robuste, F. (2008). "Optimal road pricing for quality service". In Proceedings of the transportation research board 87th annual meeting. Washington, DC, 2008.

SCAG Sothern California Association of Governments, (2005) Goods Movements in Sothern California: The Challenge, The Opportunity, and The Solution. http://www.scag.ca.gov/goodsmove/pdf/GoodsmovePaper0905.pdf [accessed on July $\underline{\left.29^{\mathrm{th}}\right]}$

Schuitema, G., Steg, L., \& Vlek, C. (2007). "Are pricing policies effective to change car use?". IATSS Research, 31(1), 21-31.

Sharp, C., Button, K.,\&Deadman,D. (1986). "The economics of tolled road crossings". Journal of Transport Economics and Policy, 20(2), 255-274.

SHRP2 Second Strategic Highway Research Program (2011), http://www.trb.org/StrategicHighwayResearchProgram2SHRP2/Blank2.aspx [accessed on July $\left.29^{\text {th }}\right]$ 
Slovic, P. (1972). From Shakespeare to Simon: Speculations-and Some Evidence-About Man's Ability to Process Information. Research Monograph 12, Oregon Research Institute.

Small, K. A., \& Verhoef, E. T. (2007). "The economics of urban transportation”. London: Routledge.

Small, K. A., \& Yan, J. (2001). "The value of value pricing of roads: Second-best pricing and product differentiation". Journal of Urban Economics, 49(2), 310-336.

Small, K. A., Winston, C., \& Yan, J. (2006). "Differentiated pricing, express lanes, and carpools: Exploiting heterogeneous preferences in policy design". Brookings-Wharton Papers on Urban Affairs, 7, 53-96

Small, K., Winston, C. and Yan, J. (2005), "Uncovering the distribution of motorists' preferences for travel time and reliability”, Econometrica, Vol. 73, pp. 1367-1382.

Smith, A., Eriksson, E., \& Lindberg, P. (1994). "Existence of optimal tolls under conditions of stochastic user-equilibria". In B. Johansson \& L. Mattsson (Eds.), Road pricing: Theory, empirical assessment and policy (pp. 65-87). Dordrecht: Kluwer.

Strasbourg, France

Supernak, J., Kaschade, C., \& Steffey, D. (2003). Dynamic value pricing on I-15 in San Diego: Impact on travel time and its reliability.Transportation Research Record, 1839, $45-54$.

Standard and Poor's (2005) http://www.standardandpoors.com/ratings/transportation/

Szeto, W. Y., \& Lo, H. K. (2006). "Dynamic traffic assignment: Properties and extensions". Transportmetrica, 2(1), 31-52.

SWRI Southwest Research Institute (2010), http://sunguide.datasys.swri.edu [accessed on July $\left.29^{\text {th }}\right]$

TRB (2005). "International perspectives on road pricing". In TRB conference Proceedings, Transportation Research Board (Vol. 34). Washington, DC.

Tu H., J. van Lint, and H. van Zulen, (2007) Travel Time Reliability Model on Freeways, $87^{\text {th }}$ Annual Meeting of the Transportation Research Board, Washington D.C.

Tu, H., van Lint, H. and van Zulen, (2008), The Effects of Traffic Accidents on Travel Time Reliability. Proceedings of the $11^{\text {th }}$ International IEEE Conference on ITS, Beijing, China. 
Van Lint J., and H. van Zulen, (2005), Monitoring and Predicting Freeway Travel Time Reliability, $84^{\text {th }}$ Annual Meeting of the Transportation Research Board, Washington D.C.

Viegas, J. M. (Ed.) (2005). "Interurban road charging for trucks in Europe". In Research in transportation economics (Vol. 11). Amsterdam: Elsevier.

Vovsha P., W. Davison and R. Donnelly (2005). "Making the State of the Art the State of the Practice: Advanced Modeling Techniques for Road Pricing". Proceedings of the xpert Forum on Road Pricing and Travel Demand Modeling, VA. pp. 95-122

Wardman, M. (1998). "The value of travel time: A review of British evidence". Journal of Transport Economics and Policy 32: 285-316.

Wardrop, J. G. (1952). "Some theoretical aspects of road traffic research". Proceedings of the Institution of Civil Engineers, 2(1), 325-362.

Washbrook, K., Haider, W., \& Jaccard, M. (2006). "Estimating commuter mode choice: A discrete choice analysis of the impact of road pricing and parking charges". Transportation, 33(6), 621-639.

Weinstein, A., \& Sciara, G.-C. (2006). "Unraveling equity in HOT lane planning: A view from practice". Journal of Planning Education and Research, 26(2), 174-184.

WSDOT, (2011) Accountability and Performance Information. http://www.wsdot.wa.gov/Traffic/Seattle/TravelTimes/reliability/ [accessed on July $29^{\text {th }}$ ]

Xin, W., Hourdos, J., Michalopoulos, P. (2006). "Preprocessing Volume Input Data for Improved Traffic Simulation". In Transportation Research Record: Journal of the Transportation Research Board, No. 1965, Transportation Research Board of the National Academies, Washington D.C, pp. 192-200.

Yamamoto, T., Fujii, S., Kitamura, R., \& Yoshida, H. (2000). "Analysis of time allocation, departure time, and route choice behavior under congestion pricing". Transportation Research Record, 1725, 95-101.

Yang, H., \& Huang, H. J. (2005). Mathematical and economic theory of road pricing.

Yin, Y., \& Yang, H. (2004). "Optimal tolls with a multiclass, bi criterion traffic network equilibrium”. Transportation Research Record, 1882, 45-52.

Ying, J. (2005). "Sensitivity analysis based method for optimal road network pricing". Annals of Operations Research, 133(1-4), 303-317.

Ying, J., \& Ando, R. (2007). “On the effects of Central Japan expressway's commuter toll discount policy in Nagoya area”. Tsinghua Science and Technology, 12(1), 151-157. 
Zhang, L. and Levinson, D. (2008), "Determinants of route choice and the value of traveler information: A field experiment.", Transportation Research Record: Journal of the Transportation Research Board, Vol. 2086, pp. 81-92.

Zhang, X., Yang, H., \& Huang, H. J. (2008). "Multiclass multicriteria mixed equilibrium on networks and uniform link tolls for system optimum". European Journal of Operational Research, 189(1), 146-158.

Zhao, Y., \& Kockelman, K. M. (2006). "On-line marginal-cost pricing across networks: Incorporating heterogeneous users and stochastic equilibria". Transportation Research Part B, 40(5), 424-435.

Zhao, Y., Kockelman, K. M., \& Karlstrom, A. (2008).”Welfare calculations in discrete choice settings: Role of error term correlation". In Proceedings of the transportation research board 87th annual meeting. Washington, DC. 
VITA

PATRICIO ALVAREZ

\section{EDUCATION}

1990 - $1996 \quad$ B.S. in Civil Engineering

University Federico Santa Maria, Valparaiso, Chile

2003 - 2004 Diploma in Pedagogy

University of Concepcion, Concepcion, Chile

2006 - $2008 \quad$ M.S., Civil Engineering

Florida International University, Miami, Florida

2008 - 2012 Doctoral Candidate in Civil Engineering

Florida International University, Miami, Florida

\section{EMPLOYMENT}

1996 - 2000 Design Engineer

DARE, Valparaiso, Chile

2000-2006 Lecturer

Department of Civil and Environmental Engineering

University of Bio-Bio, Concepcion, Chile

2006-2008 Teacher Assistant

Department of Department of Civil and Environmental Engineering

Florida International University, Miami, Florida

2008-2012 Graduate Research Assistant

Lehman Center for Transportation Research

Florida International University, Miami, Florida

\section{AFFILIATIONS}

- $\quad$ Student member, Chi Epsilon Honor Society, 2008 - Present

- $\quad$ Student member, Institute of Transportation Engineers, 2006 - Present

- $\quad$ Student member, American Society of Civil Engineers, 2006 - Present 


\section{PUBLICATIONS AND PRESENTATIONS}

- Alvarez P. and M.Hadi, "Time-Variant Travel Time Distributions and Reliability Metrics and their Utility in Reliability Assessments". Presented at the $91^{\text {st }}$ Annual Meeting of the Transportation Research Board, National Research Council, Washington D.C., January 2012.

- Hadi M., C. Zhan, P. Alvarez and A. Krishnamurthy, "Interfacing Simulation Models and Traffic Management Center Operations". Presented at the 2011 ITS World Congress, Orlando FL, October 2011

- Zhan C., P. Alvarez, M. Hadi and Y.Xiao, "Software-In-the-Loop Simulation in Support of Evaluating Traffic Management Center Software". Presented at the $90^{\text {th }}$ Annual Meeting of the Transportation Research Board, National Research Council, Washington D.C., August 2010.

- Hadi M. Zhan C., P. Alvarez, "Traffic Management Simulation Development", Final Research Report, Prepared for FDOT, September 2008.

- Alvarez P. and M.Hadi, "Calibrating Microscopic Simulation Models for Incident Conditions Using ITS Data". Presented at the Summer Meeting of the Traffic Flow Theory and Characteristics Committee, Annecy, France, July 2010.

- Alvarez P., M. Hadi and C. Zhan, "Using Intelligent Transportation Systems Data Archives for Traffic Simulation Applications". Transportation Research Records Journal of the Transportation Research Board, Washington D.C. August 2010

- Alvarez P., M. Hadi and C. Zhan, "Using Intelligent Transportation Systems Data Archives for Traffic Simulation Applications". Presented at the $89^{\text {th }}$ Annual Meeting of the Transportation Research Board, National Research Council, Washington D.C., January 2010.

- $\quad$ Hadi M., Y. Xiao, H. Ozen, P. Alvarez, "Evaluation Tools to Support ITS Planning Process: Development of a Sketch Planning Tool in FSUTMS/Cube Environment", Final Research Report, Prepared for FDOT, October 2008.

\section{HONORS AND AWARDS}

- $\quad$ Bill McGrath Transportation Scholarship, ITE District 10, 2010

- Best Student Chapter Award, ITE District 10, while serving as Chapter President, 2010

- Outstanding Leadership Award, Department of Civil and Environmental Engineering, Florida International University, 2010

- $\quad$ Spring Book Scholarship, ITE Gold Coast Chapter, 2010

- $\quad$ Francis B. Francois Award, AASHTO, 2009

- Outstanding Leadership Award, Department of Civil and Environmental Engineering, Florida International University, 2009

- Anne Brewer Scholarship, ITS Florida, 2008

- Outstanding Master Student, College of Engineering and Computing, Florida International University, 2008 